\title{
THE CLASSIFICATION OF FINITE SIMPLE GROUPS
}

\section{SIMPLE GROUPS AND LOCAL ANALYSIS}

\author{
BY DANIEL GORENSTEIN ${ }^{1}$
}

\section{Dedication}

In memory of Richard Brauer, for his pioneering studies of finite simple groups.

It is indeed unfortunate that Richard Brauer did not live to see the complete classification of the finite simple groups. He had devoted the past thirty years largely to their study and it is difficult to overestimate the impact he made on the subject. Early on, he realized the intimate relationship between the structure of a group and the centralizers of its involutions (elements of order 2). He established both qualitative and quantitative connections. As an example of the first, he showed by an elementary argument that there are at most a finite number of simple groups with a specified centralizer of an involution [30]; and of the second, he proved that if the centralizer of an involution in a simple group $G$ is isomorphic to the general linear group $G L(2, q)$ over the finite field with $q$ elements, $q$ odd, then either $G$ is isomorphic to the three-dimensional projective special linear group $L_{3}(q),{ }^{2}$ or else $q=3$ and $G$ is isomorphic to the smallest Mathieu group $M_{11}$ of order $8 \cdot 9 \cdot 10 \cdot 11$ [27], [28]. This last result, which Brauer announced in his address at the International Congress of Mathematicians in Amsterdam in 1954, represented the starting point for the classification of simple groups in terms of the structure of the centralizers of their involutions. Moreover, it foreshadowed the basic fact that conclusions of general classification theorems would necessarily include sporadic simple groups as exceptional cases $\left(M_{11}\right.$ being the sporadic group of least order).

The methods which Brauer used were almost entirely representationtheoretic and character-theoretic. In the middle 1930s he introduced and developed the concept of modular characters of a finite group. He soon realized the power of these ideas, which played an instrumental role in his proof of the Artin conjecture on $\mathcal{L}$-series in algebraic number fields. Likewise he saw that these techniques provided a powerful tool for investigating simple groups. From the middle 1940s until his death, Brauer systematically devel-

Received by the editors May, 1978.

AMS (MOS) subject classifications (1970). Primary 20D05, 20-02.

${ }^{1}$ Partially supported by National Science Foundation.

${ }^{2}$ The $n$-dimensional projective special linear group $L_{n}(q)=\operatorname{PSL}(n, q)$ over the finite field $G F(q)$ with $q$ elements is the image (modulo scalar matrices) of the group $\operatorname{SL}(n, q)$ (the special linear group) of all $n \times n$ matrices of determinant 1 with entries in $G F(q)$. 
oped the general theory of modular characters and blocks of characters, which he subsequently applied to a variety of classification questions. For me at least, the culmination of these applications occurred in a joint paper with Brauer, and J. L. Alperin [3], [4] in which we determined all simple groups with quasi-dihedral ${ }^{3}$ or wreathed ${ }^{4}$ Sylow 2-subgroups (the answer being $L_{3}(q)$, $q$ odd, $U_{3}(q), q$ odd $\left(U_{3}(q)=P S U(3, q)\right.$, denoting the three-dimensional projective special unitary group over the field $G F(q))$ or $M_{11}$ ). In the character-theoretic portions of the argument, about 100 pages in length, Brauer gives an unmatched virtuoso performance, bringing to bear all the insights about blocks of characters which he had developed over the years.

Brauer's article in this journal, based on a lecture at the University of Connecticut in the fall of 1976, gives a clear picture of the kind of questions about finite groups which continually fascinated him. The field has grown enormously from the 1940s, when Brauer was a lone figure studying simple groups, to the present time, when there are perhaps as many as 300 mathematicians scattered about the world investigating some aspect of them. But long after the simple groups are completely classified, the questions Brauer has raised about their representations and characters will continue to provide a strong stimulus for research in finite group theory.

ACKNOWLEDGEMENTS. I am extremely indebted to my colleagues Richard Lyons, Michael O'Nan, and Charles Sims for their assistance with many of the results described in this article, especially with the details of the sporadic groups. In particular, the discussion of the computer construction of sporadic groups derives directly from handwritten notes of Sims. Also my thanks to J. L. Alperin, Michael Aschbacher, Robert Gilman, George Glauberman, David Hunt, and Robert Griess for additional help they have given me. My extra thanks to Alperin, Gilman, Griess, and Lyons, who painstakingly read the entire manuscript, making innumerable improvements in the text.

\section{Table of Contents for Part I}

\section{Dedication}

Acknowledgements

Chapter I. Introduction

1. From character theory to local analysis

2. The status of the classification

3. Why the extreme length?

4. Some standard terminology and results

5. The shape of the proof

6. The four phases of the classification

\footnotetext{
${ }^{3}$ A 2-group $S$ is quasi-dihedral if $S$ is generated by elements $x, y$ subject to the relations $x^{2}=y^{2^{n}}=1, x^{-1} y x=y^{-1+2^{n-1}}, n \geqslant 3$.

${ }^{4} S$ is wreathed if $S$ is generated by elements $x, y, z$ subject to the relations $x^{2^{n}}=y^{2^{n}}=z^{2}=1$, $x y=y x, z^{-1} x z=y, z^{-1} y z=x, n \geqslant 2$.
} 
Chapter II. The known simple groups

1. The groups of Lie type

2. The Mathieu groups

3. Janko's first group

4. Sporadic groups from centralizers of involutions

5. Computer construction of sporadic groups

6. Sporadic groups as rank 3 permutation groups

7. Transpositions and the Fischer groups

8. The Leech lattice and the Conway groups

9. Concluding remark

Chapter III. Recognition theorems

1. The groups of Lie type

2. Doubly transitive groups

3. The alternating groups

4. The sporadic groups

Chapter IV. General techniques of local analysis

1. Strong embedding

2. Signalizer functors

3. $k$-balanced groups

4. $L$-balance

5. $p$-fusion

6. Stability and characteristic subgroups for odd primes

7. The Bender method, small class Sylow 2-groups, and strong closure

8. Product fusion and strong closure

9. Weak closure and trivial intersection sets

10. Factorizations and $3^{\prime}$-groups

11. Failure of factorization

12. Pushing up theorems and blocks

13. General properties of $K$-groups 


\section{CHAPTER I. INTRODUCTION}

1. From character theory to local analysis. Brauer's character-theoretic techniques turned out to be ideally suited for investigating "small" simple groups: ${ }^{5}$ linear groups of low dimension, alternating groups of low degree, groups with very restricted Sylow 2-subgroups (e.g., quaternion, ${ }^{6}$ dihedral, quasi-dihedral, wreathed, abelian, etc.). This was very fortuitous, since at the outset of the study of simple groups, it was obviously most natural to focus on the smallest ones. Moreover, these methods were so effective that in the early years there was a strong conviction that character theory would remain a principal tool-if not the primary one-for analyzing simple groups.

However, even in treating small simple groups, the method had a serious drawback: to use it to determine the structure of a group $G$, one required very precise hypotheses on at least one subgroup $H$ of $G$ (e.g., the centralizer of an involution). When such conditions prevailed, it was possible to relate the characters and modular characters of $H$ with those of $G$ and use this connection to obtain information about $G$. This was the thrust of the Brauer methods.

The difficulty occurs if one asks a broad enough question, for then one cannot assert a priori that any critical subgroup $H$ of $G$ has a very restricted structure. Let us consider, for example, the problem of determining all simple groups $G$ of order $p^{a} q^{b} r^{c}, p, q, r$ primes with $p<q<r$, which Brauer discusses briefly in his article. (In view of the classical Burnside result that all groups of order $p^{a} q^{b}$ are solvable, this is a problem of natural interest.) There are eight known simple groups having orders of this form: ${ }^{7} A_{5}, A_{6}, L_{2}(7)$, $L_{2}(8), L_{2}(17), L_{3}(3), U_{3}(3)$, and $U_{4}(2)$, each of which can certainly be considered to be a "small" group (the largest order is, in fact, 25,920).

For brevity, we call these eight groups $K_{3}$-groups and call an arbitrary group of order $p^{a} q^{b} r^{c}$ a three prime-group.

Two special situations exist in which character theory can be used to determine the structure of a simple three prime-group $G$ :

(a) The centralizer of an involution of $G$ is isomorphic to (or at least "closely resembles") that of a $K_{3}$-group;

(b) The largest prime $r$ dividing the order of $G$ occurs only to the first power (or more generally, $G$ has cyclic Sylow $r$-subgroups).

If (a) holds, one takes $H$ to be the centralizer of an involution of $G$ and investigates the modular characters of $H$ and $G$ for the prime 2; while if (b) holds, one takes $H$ to be the normalizer in $G$ of a Sylow $r$-subgroup and investigates the modular characters of $H$ and $G$ for the prime $r$.

\footnotetext{
${ }^{5}$ The term simple group always refers to a nonabelian simple group.

${ }^{6} \mathrm{~A} 2$-group $S$ is (generalized) quaternion or dihedral, respectively, if $S$ is generated by elements $x, y$ subject to the relations $x^{-1} y x=y^{-1}$ and correspondingly $x^{4}=y^{2^{n}}=1, x^{2}=y^{2^{n-1}}, n \geqslant 2$, or $x^{2}=y^{2^{n}}=1, n \geqslant 1$.

7 Here $A_{n}$ is the alternating group of degree $n$ and $U_{n}(q)=\operatorname{PSU}(n, q)=$ $S U(n, q) /(\bmod$ scalars) is the projective special unitary group over $G F(q)$. (See Chapter II for the definition of $S U(n, q)$.)
} 
We can view conditions (a) and (b) as saying that $G$ "resembles" one of the $K_{3}$-groups. Thus character theory enables one to prove the following assertion:

If a simple three prime-group resembles a $K_{3}$-group, then, up to isomorphism, $G$ is a $K_{3}$-group.

But why should an arbitrary such simple group $G$ resemble a $K_{3}$-group? Why cannot the centralizer of any of its involutions have arbitrarily high order or its Sylow $r$-subgroup have arbitrarily complex structure? Clearly unless one can establish such a resemblance, there is no hope of proving the natural conjecture that every simple three prime-group is a $K_{3}$-group. Unfortunately character theory does not seem effective for attacking this problem.

However, general methods do exist now for treating such problems. They originate in the monumental paper of Feit and Thompson which proved that all groups of odd order are solvable [57]. It is in this paper that what has come to be called local group-theoretic analysis reached full development. (Intimations appear in Thompson's doctoral thesis, in which he proved the well-known Frobenius conjecture that a finite group which admits an automorphism of prime period leaving only the identity fixed is necessarily nilpotent [197].)

It is immediate that if $G$ is a counterexample of least order to the solvability of groups of odd order, then $G$ must be simple and each of its proper subgroups is solvable. To establish the theorem, a contradiction must be derived from these conditions. Suzuki made the first significant breakthrough in the problem, treating the special case in which the centralizer of every nonidentity element of $G$ is abelian [187]. Under this assumption, it is very easy to determine the structure and embedding of every maximal subgroup $M$ of $G$ :

(1) $M=A K$, where $K$ is an abelian normal subgroup of $M, A$ is cyclic, $A \neq 1$, and every nontrivial element of $A$ induces by conjugation an automorphism of $K$ leaving only the identity fixed ( $M$ is an example of a Frobenius group with kernel $K$ and complement $A$ );

(2) $K$ has order relatively prime to its index in $G$ (one says that $K$ is a Hall $\pi$-subgroup of $G$ for some set of primes $\pi$ ); and

(3) if $g \in G$, then $g^{-1} K g \cap K=1$ or $K(K$ is said to be a trivial intersection set (T. I. set) in $G$ ).

Under these very restricted conditions, the theory of so-called exceptional characters, developed by Brauer and Suzuki (cf. [84, §4.5]), enables one to relate certain characters of $M$ with those of $G$. This connection applies to every maximal subgroup of $G$. Choosing representatives $M_{1}, M_{2}, \ldots, M_{n}$ for the conjugacy classes of maximal subgroups and applying the procedure for each $i, 1 \leqslant i \leqslant n$, Suzuki was able to obtain information about all the characters of $G$. At this point he was able to derive a contradiction by a delicate arithmetical analysis of the character values on the elements of $G$.

In a subsequent paper [55], Feit, M. Hall, and Thompson extended Suzuki's argument to the case in which it is assumed only that the centralizer of every nonidentity element of $G$ is nilpotent. They again showed that the maximal subgroups of $G$ are Frobenius groups whose kernels are Hall $\pi$-subgroups and 
are T.I. sets in $G$. Likewise they applied exceptional character theory to reach a similar arithmetic contradiction.

Thus when Feit and Thompson attacked the general solvability problem, they had a conceptual framework for a proof: first determine the structure and embedding of all the maximal subgroups of $G$; then determine the characters of $G$ using exceptional characters; and finally, hopefully, obtain an arithmetic contradiction from this information.

However, the actual task of determining the structure and embedding of these maximal subgroups turned out to be very formidable. In the process of carrying through the analysis, Thompson introduced many ideas which have come to play a central role in the study of simple groups. In particular, he established the first so-called transitivity theorems and uniqueness theorems. Thompson's approach looked very elementary: just take normalizers (and centralizers) of various nontrivial subgroups of prime power order and analyze their relationships. These normalizers, being proper subgroups of the simple group $G$, were therefore solvable and so their general structure was given by P. Hall's classical theory of solvable groups, which deals primarily with the so-called extended Sylow theorems [104], [105] (also cf. [84, Chapter 6]). In fact, Thompson would often say that all he ever used was Sylow's theorem. But what an understatement! The truth is that the analysis required the most elaborate and ingenious study of the full subgroup structure of $G$ to achieve its objective. (A fundamental result of P. Hall and G. Higman concerning solvable groups of linear transformations of a vector space over the prime field $G F(p)$ [108] (also cf. [84, Chapter 11])-and which has since had far-reaching extensions-also had a crucial impact on Thompson.)

Some time after the Feit-Thompson theorem, Alperin introduced the term local subgroup for the normalizer in $G$ of a nonidentity subgroup of prime power order $p^{n}$ ( $p$-local if one wishes to distinguish the prime $p$ ). The term local group-theoretic analysis refers to the study of the local subgroup structure of a group $G$; its primary objective is, in general, to obtain information about the structure and embedding of (a) the maximal subgroups, (b) the centralizers of involutions, and (c) the centralizers of elements of odd prime order of the group $G$.

I would like to make a few further comments about the odd order theorem before resuming the general discussion. In the general case, it was no longer possible to prove that every maximal subgroup of $G$ was necessarily a Frobenius group-a number of other structures were possible. Moreover, only a weakened form of the T.I. property could be established for these subgroups. This created obstacles to the applicability of exceptional character theory, primarily related to the character-theoretic notion of coherence. To overcome them, Feit was forced to establish an elaborate extension of the entire Brauer-Suzuki theory of exceptional characters. Unfortunately an even greater obstacle awaited Feit and Thompson-one of the final configurations of maximal subgroups completely eluded the hoped-for arithmetic contradiction. It was a full year before Thompson found a way of eliminating this last configuration-by essentially getting down to the level of the multiplication table of the group. The resulting argument, involving exceedingly complex relations among the generators of certain critical subgroups of $G$, 
ultimately succeeded in reducing the nonexistence question for $G$ to a problem about the number of solutions of a certain equation with coefficients in a finite field, which in the end Thompson was able to resolve.

The interested reader will find a fuller outline of the proof of the odd order theorem in my book [84, \$16.2]. Although over fifteen years have passed, during which time its 255 page proof has been thoroughly analyzed and parts of it extended far beyond the original context, no major conceptual improvements have been made in it-only a great many technical simplifications in portions of the argument.

The Feit-Thompson theorem, by showing that a simple group must have even order, strikingly reinforced Brauer's contention that the structure of a simple group is intimately connected with its involutions. However, as a group of odd order has no involutions, the proof itself could furnish no direct new insights into the role of the centralizers of involutions in the study of simple groups.

Such insights were provided by the next problem that Thompson attackedthe determination of all minimal simple groups-i.e., simple groups each of whose proper subgroups is solvable [199]. Since Thompson's methods involved primarily an examination of only the local subgroups, his proof actually led to the determination of the broader class of simple groups, each of whose local subgroups is solvable (for brevity, $\mathrm{N}$-groups). The theorem asserts that a simple $N$-group is isomorphic to one of the following groups: $L_{2}(q), q>3, A_{7}, L_{3}(3), U_{3}(3), M_{11}, S z(q), q=2^{2 n+1}, n \geqslant 1$, or ${ }^{2} F_{4}(2)^{\prime}$. Here $\operatorname{Sz}(q)$ denotes the family of simple groups discovered by Suzuki, related to the 4-dimensional symplectic groups over $G F(q)$; and ${ }^{2} F_{4}(2)^{\prime}$ denotes the derived group of ${ }^{2} F_{4}(2)$, the smallest member of the family of simple groups discovered by Ree, which are related to the exceptional family $F_{4}(q), q=$ $2^{2 n+1}, n \geqslant 0$, of groups of Lie type. (The group ${ }^{2} F_{4}(2)$ itself is not simple, but its derived group, of index 2, is simple.) (See Chapter II for detailed definitions.)

Here one could see for the first time how the local analytic ideas of the odd order paper could be used and further developed to yield information about the structure of the centralizers of involutions. In particular, the fundamental concept of a strongly embedded subgroup, one of the most crucial tools of simple group theory, has its origins in the $N$-group analysis (suggestions of the idea appear in earlier works of Feit and Suzuki). My aim here is solely to explain the historical origins of local group-theoretic analysis. I shall therefore leave any discussion of the major ideas of the $N$-group paper and their generalizations to the body of the article.

Return now to the problem of classifying all simple three prime-groups $\boldsymbol{G}$. Can we hope to apply local group-theoretic techniques to study the subgroup structure of $G$ ? In the odd order and $N$-group problems, the fact that all the critical subgroups were solvable was of overriding importance. But the subgroups of $G$, being quite general three prime-groups, can certainly be nonsolvable. In fact, there is no reason why their composition factors cannot include simple three prime-groups which are themselves not $K_{3}$-groups.

We can at least eliminate the second unpleasant possibility, if instead of working with an arbitrary simple three prime-group $G$, we take $G$ to be a 
simple such group of least order which is not a $K_{3}$-group. Clearly to establish the stated conjecture, it will suffice to show that no such group $G$ exists-equivalently, that $G$ must, in fact, be a $K_{3}$-group. The advantage of dealing with a "minimal counterexample" is that if $H$ is any proper subgroup of $G$, then the (nonsolvable) composition factors of $H$ are simple three prime-groups of lower order than $G$ and so are necessarily $K_{3}$-groups.

A finite group whose composition factors are known simple groups is said to be of known type-for brevity, a $K$-group. Thus the classification of simple three prime-groups is reduced to establishing the following assertion:

If $G$ is a simple three prime-group, each of whose proper subgroups is a $K$-group, then $G$ is a $K_{3}$-group.

Therefore in asking whether local group-theoretic methods can be used in this problem, we are really asking whether the Thompson techniques for studying simple groups with solvable proper subgroups can be extended to simple groups whose proper subgroups are $K$-groups. A large part of the effort of the past fifteen years has been devoted precisely to carrying through such a generalization.

The first general classification theorem involving nonsolvable proper subgroups was the determination of all simple groups with dihedral Sylow 2-subgroups which John Walter and I obtained in the early 1960s, [94], the answer being the groups $L_{2}(q), q$ odd, $q>3$, and $A_{7}$. To carry through the analysis, we required many very specific properties of the groups $L_{2}(q)$ and $A_{7}$, since these groups could occur as sections of the proper subgroups of a minimal counterexample. Thus the proof of our theorem strongly indicated that any broad extension of the Thompson methods would involve some sort of general theory of $K$-groups related to local analysis.

The full development of these Sylow-type local group-theoretic methods has undoubtedly been the single most significant factor in the remarkable recent advances that have been made towards the complete determination of the finite simple groups. However, not far behind in importance are the internal geometric methods, introduced by Bernd Fischer in the late 1960s, which grew out of his attempt to characterize the symmetric groups by the fact that they are generated by a conjugacy class of involutions (namely, their transpositions $(i j)$ ), the product of any two of which has order 1,2 , or 3 [59], [62], [63]. The ideas involved in the solution of this problem have led not only to the discovery of several sporadic simple groups, but ultimately to the creation of new general methods for studying simple groups. These will be fully discussed in the body of the article.

Just as Suzuki and Feit are products of the Brauer tradition, so Bender, Glauberman, Goldschmidt, I, and many others are primarily disciples of Thompson; while Aschbacher, O'Nan, and Timmesfeld are largely of the Fischer school. However, in the past few years, Aschbacher has succeeded in synthesizing the Sylow and geometric analytic methods to such an extent as to create a dazzling new approach to simple group theory.

2. The status of the classification. At the present time the determination of all finite simple groups is very nearly complete. Such an assertion is obviously 
presumptuous, if not meaningless, since one does not speak of theorems as "almost proved". But the ultimate theorem which will assert the classification of simple groups is unlike any other in the history of mathematics; for the complete proof, when it is attained, will run to well over 5,000 journal pages! Moreover, it is very likely that at the present time more than $80 \%$ of these pages exist in either print or preprint form.

Under such circumstances, it makes more sense to view this classification as an entire field of mathematics rather than as an attempt to prove a single theorem. Furthermore, it should be emphasized that the portion of the proof already in print constitutes primarily a long series of theorems which stand in their own right, independent of whether or not a complete classification is ever achieved. Many of these represent the determination of all simple groups with some specific property (e.g., $G$ has odd order, $G$ has dihedral Sylow 2-subgroups, $G$ has abelian Sylow 2-subgroups, the 2-local subgroups of $G$ have cyclic Sylow $p$-subgroups for all odd primes, the centralizer of an involution of $G$ closely approximates that in some known simple group, etc., etc.). Many represent the development of general techniques which facilitate the study of simple groups; others involve the determination of properties of $K$-groups which are needed to carry out local analysis. A large number of others deal with a specific individual (sometimes two or three) sporadic group-its construction, some characterization, or some properties. Thus finite simple group theory really is a total field of mathematics; and, in general, each separate result bears the same relationship to the entire subject as does, say, a single theorem in differential topology.

Out of the continued study of simple groups, there has gradually evolved a specific program for obtaining their ultimate classification. In the early years, many directions were pursued which, interesting in themselves, have turned out to have little bearing on the "program" (e.g., the questions of classifying groups by the number of conjugacy classes of their elements or whether a known simple group can be generated by two elements). Such questions simply do not arise in the course of proving any general classification theorem. At a later stage, considerable effort was spent looking for new sporadic groups in directions which had already produced such groups. With a few notable exceptions, this experimental approach was not successful. For example, Conway's three simple groups which arise as automorphisms of the 24-dimensional integral lattice of Leech [42] stimulated a great deal of interest in the study of integral lattices and their automorphism groups. Unfortunately no other groups have been discovered in this way. This effort gave considerable insight into integral representations of groups, but apart from one important paper of Feit [54], it has had little impact on the development of simple group theory.

In recent years, as the full outline of the proof has become more sharply delineated, investigations of simple groups have become increasingly focused. To crystalize these developments, I gave a short series of lectures at the University of Chicago in June, 1972 in which I presented a detailed sixteen step program for carrying out the classification of simple groups.

I am including the manuscript of these talks as an appendix to this article, for it is very instructive. Indeed, it shows the extent to which it was already 
possible by 1972 to predict the final shape of the classification theorem. New insights have certainly forced modifications of individual steps in the program - for example, the key role of "tightly embedded" subgroups was missing as was the then undeveloped theory of "pushing up", and the role of the odd prime 3 for groups of characteristic 2 type was overemphasized. Likewise I did not then appreciate the far reaching impact that Fischer's approach would have on simple group theory. Nevertheless the overall program has remained essentially intact. In the last few years, as the momentum has gathered, the progress towards its full realization has been very rapid, spurred on by the astonishing sequence of fundamental results of Aschbacher, which have sharply advanced the time table for the ultimate determination of all simple groups.

The assertion that the classification is nearly complete is really a prediction that the presently available techniques will be sufficient to deal with the problems still outstanding. In its support, we cite the fact that, with one or two exceptions, all open questions are open because no one has yet examined them and not because they involve some intrinsic difficulty. Moreover, at least on the surface, each of these appears to be similar to other problems which have already been successfully attacked. The major single exception concerns the classification of so-called groups of "Ree type" which has been intensively investigated by Thompson [202] and is still not completely resolved after more than ten years (see Chapter III).

Implicit in this prediction is a belief that any as yet undiscovered simple groups will possess internal structures not unlike those of the known simple groups. This will be necessary if the already developed theory of $K$-groups is to remain intact if and when any such new groups are added to the list of simple $K$-groups.

The principal aim of this article is to present a detailed summary of the proof of the classification of simple groups, to the extent that this has been accomplished at the present time.

This is an appropriate moment to add a cautionary word about the meaning of "proof" in the present context; for it seems beyond human capacity to present a closely reasoned several hundred page argument with absolute accuracy. I am not speaking of the inevitable typographical errors, or the overall conceptual basis for the proof, but of "local" arguments that are not quite right-a misstatement, a gap, what have you. They can almost always be patched up on the spot, but the existence of such "temporary" errors is disconcerting to say the least. Indeed, they raise the following basic question: If the arguments are often ad hoc to begin with, how can one guarantee that the "sieve" has not let slip a configuration which leads to yet another simple group? Unfortunately, there are no guarantees-one must live with this reality. However, there is a prevalent feeling that, with so many individuals working on simple groups over the past fifteen years, and often from such different perspectives, every significant configuration will loom into view sufficiently often and so cannot remain unnoticed for long. On the other hand, it clearly indicates the strong need for continual reexamination of the existing "proofs". This will be especially true on that day when the final classification of simple groups is announced and the exodus, already begun, 
to more fertile lands takes place. Some of the faithful must remain behind to improve the "text". This will be one of the first major tasks of the "post classification" era.

3. Why the extreme length? There exists an often expressed feeling in the general mathematical community that the present approach to the classification of simple groups must be the wrong one-no single theorem can possibly require a 5,000 page proof! Since most of the known simple groups are finite analogues of Lie groups, one should be able to build a geometry from suitable internal properties of a simple group $G$ and then determine $G$ from its action on this geometry. This is the most frequently suggested alternate approach. If successful, it would reduce the classification to the presumably more tractable problem of classifying the corresponding geometries. After all, this is exactly how the classification of complex simple Lie algebras proceeds (and with it the classification of simple Lie groups): the entire problem is reduced to the analysis of connected "Dynkin diagrams"; equivalently to the solution of a certain question about sets of vectors in complex $n$-space. Just as there are five exceptional complex simple Lie algebras and Lie groups- $G_{2}, F_{4}, E_{6}, E_{7}, E_{8}$-so one could vizualize certain exceptional geometries arising from the finite simple groups, each of which would lead to one of the sporadic groups.

The suggestion is so plausible, why has it not been possible to proceed along such lines to study simple groups? Let me attempt an answer. Just as in the special problem of determining all simple three prime-groups, discussed above, so the general classification theorem reduces at once to establishing the following assertion:

If $G$ is a finite simple group each of whose proper subgroups is a $K$-group, then $G$ itself is a $K$-group.

Let $G^{*}$ be a completely arbitrary $K$-group, as far from being simple as you can conceive. Is there any a priori reason why our simple group $G$ above should not have the identical lattice of proper subgroups as $G^{*}$ ? If this is so, then the internal structure of $G$ bears no resemblance to that of any simple group, but rather is equivalent to that of an arbitrary $K$-group. Hence if we attempted to construct a geometry directly out of $G$, the resulting geometry would be identical to one constructed out of $G^{*}$. Thus, if the suggested approach were to be followed at the very outset, it would necessarily generate as many distinct geometries as there are finite $K$-groups. The classification of such geometries would appear to be a hopeless task.

The implication of this discussion is the following: One cannot expect a geometric approach to be effective until after one uses the simplicity of $G$ to show that its internal structure is, in fact, much more restricted than that of the general $K$-group $G^{*}$-indeed, until one shows that its internal structure "resembles" that of a simple $K$-group. It is precisely this resemblance which local group-theoretic analysis has as its primary objective. Once $G$ has been shown to resemble internally a simple $K$-group $G^{*}$, the proof that $G$ is actually isomorphic to $G^{*}$ can be regarded as involving the construction of a "geometry". This chapter of finite group theory, which deals with so-called 
"recognition" theorems, is very closely linked to the Lie theory. Thus it is not that finite group-theorists have ignored the geometric approach to the classification of simple groups, but rather that this phase occurs at a late stage in the analysis.

This situation has no analogue in the classification of complex simple Lie algebras since there the nondegeneracy of the Killing form gives a sharp criterion for semisimplicity, enabling one to reduce the classification of semisimple and simple Lie algebras to the above-mentioned geometric problem. However, there is some evidence that the as yet uncompleted classification of simple Lie algebras over fields of characteristic $p \neq 0$ may very well involve an analogue of local group-theoretic analysis.

If, as we are suggesting, the local analytic approach to the determination of the simple groups is the only available one, then the question of the length of the classification proof reduces to the amount of work involved in settling the following two problems:

(A) Proving that $G$ resembles internally a simple $K$-group $G^{*}$.

(B) Proving that whenever $G$ does resemble such a group $G^{*}$, then $G$ must be isomorphic to $G^{*}$.

Clearly answers to (A) and (B) in turn involve the following two subsidary questions:

I. How uniform are the internal structures of simple $K$-groups? In other words, how many distinct "types" of simple $K$-groups must one consider?

II. For those groups $G$ which will end up isomorphic to a simple $K$-group $G^{*}$ of a given type, can the analysis under (A) and (B), respectively, be carried out uniformly for each of the given types?

At the minimum it seems necessary to consider the following families of $K$-groups to be of distinct types (the known simple groups will be described in detail in Chapter II).

1. The groups of prime order,

2. The alternating groups,

3. The groups of Lie type over $G F(q), q$ odd,

4. The groups of Lie type over $G F(q), q$ even,

5. The 26 sporadic groups.

The distinction of 3 and 4 is an essential one, for in a group $G^{*}$ of Lie type defined over $G F(q)$, an involution of $G^{*}$ corresponds to a semisimple element or a unipotent element according as $q$ is odd or even. Correspondingly the centralizers of these involutions have totally distinct structures (see §5).

Moreover, in treating many situations, one must further subdivide the groups of Lie types as follows:

a. The classical groups: linear, symplectic, orthogonal,

b. The exceptional groups: $G_{2}, F_{4}, E_{6}, E_{7}, E_{8}$,

c. The "algebraic twisted" groups: unitary groups, triality $D_{4}$, twisted $E_{6}$ (the finite analogues of the orthogonal groups $O_{2 n}^{-}(\mathrm{C})$ will also be included under c),

d. The "nonalgebraic twisted" groups: Suzuki groups, Ree groups of characteristic 3, Ree groups of characteristic 2.

Although some collections of sporadic groups arise out of a single context (the first two Mathieu groups $M_{11}, M_{12}$; the remaining three Mathieu groups 
$M_{22}, M_{23}, M_{24}$; Janko's second and third groups $J_{2}, J_{3}$; the three Conway groups .1, .2, .3; the first three Fischer groups $M(22), M(23), M(24)^{\prime}$; and the last two Fischer groups, the so-called "baby monster" $F_{2}$ and "monster" $F_{1}$ ), from the point of view of their classification by internal properties, the 26 sporadic groups are best considered to be of distinct types.

The above subdivision gives rise to roughly 40 distinct types of simple $K$-groups (over half representing individual groups). This means that at various stages in carrying through the analysis of (A) and (B) above, up to 40 distinct cases may have to be treated.

In addition, there are often many other cases which must be considered, unrelated to any known simple group. For example, the final configuration of the odd order paper is as "tight" as any in which $G$ has an internal structure resembling a simple $K$-group $G^{*}$. Thus it is not so far-fetched to say in this case that the internal structure of $G$ resembles that of a nonexistent simple group. The point is that the analysis one is often forced to take to eliminate a particular configuration is in spirit not unlike that which one carries out in "real" cases, in showing either that $G$ internally resembles some simple $K$-group $G^{*}$, or, at a later stage, that $G$ is isomorphic to $G^{*}$.

As already indicated, special methods are required for investigating small simple groups. But beyond that, the analysis in these cases is extremely complicated and each specific problem involves its own special configurations and its own, ad hoc, arguments. For example, the determination of the simple groups with quasi-dihedral or wreathed Sylow 2-subgroups runs to over 300 pages. Yet, apart from the actual result itself and a few general ideas of the proof, no specific proposition or lemma has ever been utilized in any other classification problem.

As a consequence, it is very likely that as much as half of the entire proof of the ultimate classification thoerem will be devoted to small groups. This analysis, which is very nearly complete at the present time, already covers nearly 3,000 journal pages plus a considerable amount of computer time (to prove the existence and uniqueness of certain sporadic groups). Given all the new methods recently developed, one can certainly expect some simplification in the already existing proofs of earlier results, However, because of the decidedly individual nature of these arguments, it would appear to be very difficult, if not impossible, to develop a uniform approach that would condense them by a factor of, say, five.

Here then is an "insider's" explanation of the enormous length of the classification proof.

4. Some standard terminology and results. I should like to conclude this introduction by making more precise the major subdivision of the study of simple groups as it has evolved to the present time. To do so requires a considerable amount of standard notation and terminology. We shall therefore review this now together with some equally standard basic results which we shall need throughout the article. I apologize for the length of the list, but it is impossible to discuss anything meaningfully without a reasonable vocabulary. The unfamiliar reader will probably do best to assimilate this material as it comes up in the text. 
We let $X$ be an arbitrary finite group.

$Y<X$ means $Y$ is a subgroup of $X$.

$Y<X$ means $Y$ is a proper subgroup of $X$; i.e., $Y<X$ and $Y \neq X$.

$Y \triangleleft X$ means $Y$ is a normal subgroup of $X$; i.e., $Y$ is invariant under conjugation by all elements of $X$ (equivalently, under all inner automorphisms of $X$ ).

$Y \triangleleft \triangleleft X$ means $Y$ is a subnormal subgroup of $X$; i.e., $Y=Y_{1} \triangleleft Y_{2} \triangleleft Y_{3}$ $\triangleleft \cdots \triangleleft Y_{n}=X$ for suitable subgroups $Y_{i}$ of $X, 1<i<n$.

$Y$ char $X$ means $Y$ is a characteristic subgroup of $X$; i.e. $Y$ is invariant under all automorphisms of $X$.

$Y$ is a section of $X$ means $Y=A / B$, where $A, B<X$ and $B \triangleleft A$.

$Y$ is involved in $X$ means $Y$ is isomorphic to a section of $X$.

$Y$ covers a section $A / B$ of $X$ means $Y \leqslant X$ and $A=B(Y \cap A)$.

$|S|$ denotes the cardinality of the set $S$. In particular, $|Y|$ is the order of any subset $Y$ of $X$.

$|X: Y|$ denotes the index of the subgroup $Y$ of $X$; i.e. $|X| /|Y|$.

$\langle Y\rangle$ denotes the unique smallest subgroup of $X$ containing the subset $Y$ of $X .\langle Y\rangle$ is the intersection of all subgroups of $X$ containing $Y$. If $\langle Y\rangle=X, Y$ is said to generate $X$ or to be a set of generators of $X$.

$C_{X}(Y)$ denotes the centralizer in $X$ of the subset $Y$ of $X$; i.e., the set of all elements of $X$ which commute elementwise with $Y$.

$N_{X}(Y)$ denotes the normalizer in $X$ of the subgroup $Y$ of $X$; i.e., the unique largest subgroup of $X$ containing $Y$ as a normal subgroup.

$\left\langle Y^{X}\right\rangle$ denotes the normal closure in $X$ of the subset $Y$ of $X$; i.e., the subgroup generated by all conjugates of $Y$ in $X$.

$Z(X)$ denotes the center of $X$.

$S y l_{p}(X)$ denotes the set of Sylow $p$-subgroups of $X$ for any prime $p$.

Sol $(X)$ denotes the unique largest normal solvable subgroup of $X$.

$F(X)$ denotes the Fitting subgroup of $X$, the unique largest normal nilpotent subgroup of $X . F(X)$ is the join of all normal nilpotent subgroups of $X$.

$\phi(X)$ denotes the Frattini subgroup of $X$, which is the intersection of the maximal subgroups of $X$.

$\operatorname{Aut}(X)$ denotes the automorphism group of $X$.

1 denotes the identity subgroup and element of $X$ as well as the trivial group with one element.

$X$ acts on the group $Y$ means that there is a homomorphism of $X$ into Aut $(Y)$. In particular, if $Y \nabla X$, then $X$ acts on $Y$ by conjugation.

$A B=\{a b \mid a \in A, b \in B\}$ for any subsets $A, B$ of $X$. The notation extends in a natural way to any finite number of subsets of $X$.

$A^{B}=\left\{b^{-1} a b \mid a \in A, b \in B\right\}$ for any subsets $A, B$ of $X$.

$[a, b]=a^{-1} b^{-1} a b=a^{-1} a^{b}$ for any elements $a, b \in X$.

$[A, B]=\langle[a, b] \mid a \in A, b \in B\rangle$ for any subgroups $A, B$ of $X$.

$[a, b, c]=[[a, b], c]$ for any elements $a, b, c \in X$. Inductively, one defines commutators of arbitrary length; and likewise for sequences of subgroups.

$X^{\prime}=[X, X]$ denotes the commutator subgroup of $X$. If $X=X^{\prime}, X$ is perfect.

$Z_{n}$ denotes a cyclic subgroup of order $n$.

$Q_{2^{n}}$ denotes a (generalized) quaternion group of order $2^{n}, n \geqslant 3$. 
$D_{2 n}$ denotes a dihedral group of order $2 n, n \geqslant 2$.

An abelian $p$-group $X$ is homocyclic if it is the direct product of cyclic subgroups of the same order $p^{m}$. If $m=1, X$ is called elementary. We write $E_{p^{n}}$ for an elementary abelian $p$-group of rank $n$. Note that $E_{p^{n}}$ can be identified with an $n$-dimensional vector space over the prime field $G F(p)$. Note also that, for example, $E_{8}$ and $Z_{2} \times Z_{2} \times Z_{2}$ are isomorphic groups.

A group isomorphic to $Z_{2} \times Z_{2}$ is called a four group.

$\Sigma_{n}$ denotes the symmetric group of all permutations of the set $\{1,2, \ldots, n\}$.

$X$ is a central product of subgroups $A, B$ if $X=A B$ and $[A, B]=1$. The product is direct if, in addition, $A \cap B=1$. In the latter case, we write $X=A \times B$. On the other hand, if $A \cap B \neq 1$ (note that $A \cap B \leq Z(A) \cap$ $Z(B)$ ), we write $X=A * B$. These concepts and notation extend in a natural way to finite products of subgroups of $X$.

If $A$ is a group and $B$ a subgroup of $\operatorname{Aut}(A)$, the semidirect product or split extension of $A$ by $B$ is a group of the form $A^{*} B^{*}$ with $A^{*} \cong A, B^{*} \cong B$, $A^{*} \cap B^{*}=1, A^{*} \nabla A^{*} B^{*}$, and the action of $B^{*}$ on $A^{*}$ by conjugation being determined by the action of $B$ as a group of automorphisms of $A$. One identifies $A^{*}$ with $A, B^{*}$ with $B$ and writes $A \cdot B$ (or simply $A B$ ) for the semidirect product.

To adjoin an automorphism $\beta$ to a group $A$ or to extend $A$ by $\beta$ means simply to form the group $A \cdot\langle\beta\rangle$.

The wreath product of a group $A$ by a group $B$, denoted by $A / B$, although conceptually clear, is cumbersome to express. One forms the direct product $A^{*}$ of $n=|B|$ copies of $A$, indexed from 1 to $n$, and identifies $B$ in a natural way with a subgroup of $\Sigma_{n}{ }^{8}$. The permutation action of $B$ on the set $\{1,2, \ldots, n\}$ is then used to induce an automorphism action of $B$ on $A^{*}$ : namely, for $\left(a_{1}, a_{2}, \ldots, a_{n}\right) \in A^{*}, a_{i} \in A, 1 \leqslant i \leqslant n$, and $b \in B$, one sets

$$
\left(a_{1}, a_{2}, \ldots, a_{n}\right)^{b}=\left(a_{1}^{\prime}, a_{2}^{\prime}, \ldots, a_{n}^{\prime}\right) \text {, where } a_{i}^{\prime}=a_{j} \text { if } i=j^{b}, 1 \leqslant i, j \leqslant n \text {. }
$$

Finally, using this action, one defines the wreath product $A / B$ to be the semidirect product $A^{*} \cdot B$.

For example, if $|B|=2$, then $A \int B$ consists of the direct product of two copies of $A$, interchanged under conjugation by the involution of $B$. In this terminology, our earlier definition of a wreathed 2-group is just $Z_{2^{m}} \int Z_{2}$ for some integer $m>2$.

If $\pi$ is a set of primes, $\pi^{\prime}$ denotes the complementary set of primes.

$O_{\pi}(X)$ denotes the unique largest normal subgroup of $X$ whose order is divisible only by primes in the set $\pi$. It is the join of all normal subgroups of $X$ with this property.

$O^{\pi}(X)$ denotes the unique smallest normal subgroup $Y$ of $X$ such that $X / Y$ has order divisible only by primes in $\pi$. It is the intersection of all

\footnotetext{
${ }^{8}$ If $X=\left\{x_{1}, x_{2}, \ldots, x_{n}\right\}$ is a group, then for $x \in X$, the map $\phi_{x}: x_{i} \mapsto x_{i} x, 1 \leqslant i \leqslant n$, is a permutation of the set $\left\{x_{1}, x_{2}, \ldots, x_{n}\right\}$. If we identify this set with $\{1,2, \ldots, n\}$, then the map $\theta: x \mapsto \phi_{x}$ for all $x \in X$, is an isomorphism of $X$ with a subgroup of $\Sigma_{n}$. (This is Cayley's theorem.) Using $\theta$, one can identify $X$ with a subgroup of $\Sigma_{n}$.
} 
normal subgroups of $X$ such that the corresponding factor group has this property.

$O_{p^{\prime}}(X)$ is the $p^{\prime}$-core of $X$, the unique largest normal subgroup of $X$ of order relatively prime to $p, p$ any prime.

$O(X)=O_{2^{\prime}}(X)$ is the core of $X$, the unique largest normal subgroup of $X$ of odd order.

$X$ has a normal $p$-complement provided $X / O_{p^{\prime}}(X)$ is a $p$-group (whence $X=O_{p^{\prime}}(X) P$ for any $\left.P \in S y l_{p}(X)\right)$.

If $O_{p^{\prime}}(X)=1, X$ is $p$-constrained, $p$ any prime, provided $C_{X}\left(O_{p}(X)\right) \leqslant$ $O_{p}(X)$.

More generally, $X$ is $p$-constrained if $X / O_{p^{\prime}}(X)$ is. (Note that $\left.O_{p^{\prime}}\left(X / O_{p^{\prime}}(X)\right)=1.\right)$

The following additional terminology, although not so standard, will be very useful.

$g_{p}(X)$ denotes the set of elements of $X$ of order $p, p$ a prime.

$\Phi(X)=\Phi_{2}(X)$.

$y \in \mathscr{G}_{p}(X)$ is $p$-central if $y$ lies in the center of some Sylow $p$-subgroup of $X$.

Further notation will be introduced as we go along.

We conclude this section with some elementary terminology and facts about permutation groups, generators and relations, and $p$-groups.

$\Sigma(\Omega)$ denotes the symmetric group of all permutations of the (finite) set $\Omega$. Thus $\Sigma_{n}=\Sigma(\Omega)$ for $\Omega=\{1,2, \ldots, n\}$.

$A(\Omega)$ (resp. $A_{n}$ ) denotes the alternating subgroup of even permutations of $\Sigma(\Omega)\left(\right.$ resp. $\left.\Sigma_{n}\right)$.

$X$ is a permutation group on $\Omega$ if $X \leqslant \Sigma(\Omega) .|\Omega|$ is the degree of $X$.

$X$ is $k$-fold transitive on $\Omega$ if any two ordered $k$-tuples of elements of $\Omega$ can be transformed into each other by elements of $X$. One writes transitive, doubly transitive, triply transitive, etc., for 1-fold, 2-fold, 3-fold transitive, etc. Clearly $k$-fold transitivity implies $k^{\prime}$-fold transitivity for all $k^{\prime} \leqslant k$.

If $X \leqslant \Sigma(\Omega)$, then for any $\Lambda \leqslant \Omega$, the $\Lambda$-point stabilizer $X_{\Lambda}$ of $X$ is the subset of permutations of $X$ fixing each point of $\Lambda$. It is clearly a subgroup of $X$. If $X$ is $k$-fold transitive, then for any subsets $\Lambda_{1}, \Lambda_{2}$ of $\Omega$ of cardinality $k$, $X_{\Lambda_{1}}$ and $X_{\Lambda_{2}}$ are conjugate subgroups of $X$. Because of this, we speak simply of the $k$-point stabilizer of $X$ (when $X$ is $k$-fold transitive). It is determined up to conjugacy.

If $X \leqslant \Sigma(\Omega)$ is transitive on $\Omega$, it is immediate that the 1-point stabilizer has index $|\Omega|$ in $X$.

$X \leqslant \Sigma(\Omega)$ is primitive if $X$ is transitive on $\Omega$ and its 1-point stabilizer is a maximal subgroup of $X$.

$X \leqslant \Sigma(\Omega)$ has (permutation) rank $r$ if $X$ is transitive on $\Omega$ and its 1-point stabilizer has exactly $r$ orbits on $\Omega$. Thus $X$ is doubly transitive on $\Omega$ if and only if $X$ has rank 2.

A homomorphism $\phi$ of $X$ into $\Sigma(\Omega)$ is called a permutation representation of $X$ on $\Omega .|\Omega|$ is the degree of $\phi . \phi$ is $k$-fold transitive if $\phi(X)$ is $k$-fold transitive on $\Omega$.

If $X \leqslant \Sigma(\Omega)$ is transitive on $\Omega$, it is immediate that the 1-point stabilizer has index $|\Omega|$ in $X$. Thus $X / \operatorname{ker} \phi \leqslant \Sigma(\Omega)$. 
Two permutation representations $\phi$ and $\phi^{\prime}$ of $X$ on sets $\Omega, \Omega^{\prime}$ are equivalent if there exists a one-one mapping $\theta$ of $\Omega$ on $\Omega^{\prime}$ such that $\phi^{\prime}=\theta^{-1} \phi \theta$.

For $Y \leqslant X$, let $\Omega_{Y}=\left\{Y_{1}, Y_{2}, \ldots, Y_{n}\right\}$ be the set of right cosets of $Y$ in $X$. For $x \in X$, the mapping $\phi_{x}: Y_{i} \mapsto Y_{i} x, 1 \leqslant i \leqslant n$, is a permutation of $\Omega_{Y}$ and the mapping $\phi_{Y}: x \mapsto \phi_{X}$ for $x \in X$ defines a transitive permutation representation of $X$ on $\Omega_{Y}$ having $Y$ as a 1-point stabilizer. Moreover, ker $\phi_{Y}=$ $\cap_{x \in X} Y^{x}$.

Similarly let $\Omega_{Y}^{\prime}=\left\{Y_{1}^{\prime}, Y_{2}^{\prime}, \ldots, Y_{n}^{\prime}\right\}$ be the set of distinct conjugates of $Y$ in $X$. Defining $\phi_{x}^{\prime}: Y_{i} \mapsto Y_{i}^{x}, 1 \leqslant i \leqslant n$, for $x \in X$ and $\phi_{Y^{\prime}}: x \mapsto \phi_{x}^{\prime}$ for $x \in X$, $\phi_{Y}^{\prime}$ is a transitive permutation representation of $X$ on $\Omega_{Y}^{\prime}$. Moreover, $\phi_{Y}^{\prime}$ is equivalent to $\phi_{N_{X}(Y)}$. In particular, $\phi_{Y}^{\prime}$ is equivalent to $\phi_{Y}$ if $Y=N_{X}(Y)$.

The significance of these particular permutation representations can be seen from the following elementary result.

Proposition 1. Every transitive permutation representation of a group $X$ is equivalent to the transitive permutation representation on the right cosets of some subgroup of $X$.

Finally one has the following equally basic fact.

Proposition 2. The permutation representation of a group $X$ on the right cosets of $Y \leqslant X$ is doubly transitive if and only if $X=Y \cup Y x Y$ for any $x \in X-Y$.

Now for generators and relations. If $Y$ is a set of generators of $X$, a word in $Y$ is a finite formal product $a b c \ldots$ of elements $a, b, c, \ldots$ of $Y$. A word $W$ is called a relator if it represents the identity element of $X$. The statement $W=1$ is called a relation. If $P_{i}, 1 \leqslant i \leqslant n$, are relators (in $Y$ ), a word $W$ in $Y$ is derivable from the $P_{i}$, if the following operations, applied a finite number of times, transform $W$ into the empty word:

(a) Insertion of some $P_{i}$ or $P_{i}^{-1}$ between any two symbols of $W$ or before $W$ or after $W$;

(b) Deletion of some $P_{i}$ or $P_{i}^{-1}$ if it forms a block of consecutive symbols of $W$.

Clearly in such a case $W$ itself is also a relator.

If every relator (in $Y$ ) is derivable from the relators $P_{i}, 1 \leqslant i \leqslant n$, together with the trivial relators $\left\{a a^{-1} \mid a \in Y\right\}$, then the $P_{i}$ are called a complete set of defining relators and the equalities $P_{i}=1$ a complete set of defining relations for $X$. The set of generators $Y$ together with a complete set of defining relators or relations is called a presentation of $X$.

The importance of presentations is that they "characterize" the group $X$. Indeed, if $X, X^{*}$ are groups with presentations $\left(Y,\left\{P_{i}, 1 \leqslant i \leqslant n\right\}\right)$ and $\left(Y^{*},\left\{P_{i}^{*}, 1 \leqslant i \leqslant n^{*}\right\}\right.$ ) and if there exists a one-one mapping $\theta$ from $Y$ to $Y^{*}$ (whence $|Y|=\left|Y^{*}\right|$ ) which induces in the obvious way a one-one map from the set $\left\{P_{i}, 1 \leqslant i \leqslant n\right\}$ onto the set $\left\{P_{i}^{*}, 1 \leqslant i \leqslant n^{*}\right\}$ (whence $n=n^{*}$ ), then the natural extension of $\theta$ to the set of all words in $Y$ onto all words in $Y^{*}$ induces an isomorphism of $X$ on $X^{*}$.

Finally some elementary facts about $p$-groups. First, in any group $X$, the 
chain of subgroups $X, X^{\prime},\left(X^{\prime}\right)^{\prime}, \ldots$ is called the derived series of $X$; and the chain $X, X^{\prime},\left[X^{\prime}, X\right],\left[\left[X^{\prime}, X\right], X\right], \ldots$ is called the lower central series of $X$. By definition, $X$ is solvable if and only if its derived series terminates in the identity; and $\boldsymbol{X}$ is nilpotent if and only if its lower central series terminates in the identity. If $X$ is nilpotent, it is necessarily solvable. It can also be easily shown that $X$ is nilpotent if and only if it is the direct product of its Sylow subgroups. In particular, $X$ is nilpotent if $X$ is of prime power order.

If $X$ is nilpotent, the class of $X$ is 1 less than the number of distinct terms in its lower central series. Thus an abelian group is nilpotent of class 1 .

If $X$ is a $p$-group, $\Omega_{i}(X)$ denotes the subgroup of $X$ generated by all elements of $X$ of order at most $p^{i}$. Clearly $\Omega_{i}(X)$ is characteristic in $X$.

If $p^{n}$ is the maximum order of an element of $X$, then $X$ has exponent $p^{n}$. Thus if $X$ is abelian of exponent $p$, then $X$ is elementary.

The following properties of $p$-groups are very old.

Proposition 3. If $X$ is a p-group, then

(i) $X / \phi(X)$ is elementary abelian. (In particular, $X^{\prime} \leqslant \phi(X)$.)

(ii) If $\alpha$ is an automorphism of $X$ of order prime to $p$ and $\alpha$ acts trivially on $X / \phi(X)$, then $\alpha$ is the identity automorphism.

A $p$-group $X$ is special if either $X$ is elementary abelian or $X^{\prime}=\phi(X)=$ $Z(X)$ is elementary. In particular, $X$ has class 1 or 2 , Moreover, $X$ is called extra-special if $X$ is nonabelian special and $\left|X^{\prime}\right|=p$.

Extra-special $p$-groups are important in simple group theory, in large measure because of the following result of $P$. Hall [84, Theorem 5.4.9]. then

PROPOSITION 4. If a p-group $X$ has no noncyclic character abelian subgroups,

$$
X=A * B,
$$

where $A$ is extra-special (or $A=1$ ) and either $B$ is cyclic or $p=2$ and $B$ is dihedral, quasi-dihedral, or quaternion.

The structure of extra-special p-groups can be precisely determined (cf. [84, 85.5]).

Proposition 5. If $X$ is an extra-special p-group, then

$$
X=A_{1} * A_{2} * \cdots * A_{n},
$$

where each $A_{i}$ is extra-special of order $p^{3}$.

The integer $n$ is called the width of $X$. For a given $p$, there are two nonisomorphic extra-special $p$-groups of order $p^{3}$. If $p$ is odd, one of these is of exponent $p$ and the other has a maximal cyclic subgroup of order $p^{2}$; while if $p=2$, one is quaternion of order 8 and the other dihedral of order 8 . Moreover, it is easily checked that $Q_{8} * Q_{8} \cong D_{8} * D_{8}$.

We introduce the symbol $\left(Q_{8}\right)^{k}$ (resp. $\left.\left(D_{8}\right)^{k}\right)$ to denote the extra-special 2-group of width $k$, each of whose factors is $Q_{8}$ (resp. $D_{8}$ ). In view of this discussion, one can easily prove

Proposition 6. If $X$ is an extra-special 2-group of width $n$, then $X \cong\left(Q_{8}\right)^{n}$ or $\left(Q_{8}\right)^{n-1} * D_{8}$. Moreover, the latter two groups are not isomorphic. 
Finally a $p$-group $X$ with no noncyclic characteristic abelian subgroups is said to be of symplectic type.

The following considerations will explain this choice of terminology. Suppose $X$ is extra-special and set $Z(X)=\langle z\rangle, \bar{X}=X / Z(X)$. Thus $\bar{X}$ is a vector space over $G F(p)$ and $|z|=p$. We define a map

$$
\rho: \bar{X} \times \bar{X} \mapsto G F(p) \text {. }
$$

For $\bar{x}, \bar{y} \in \bar{X}$, let $x, y$ be representatives of $\bar{x}, \bar{y}$ in $X$. Then

$$
[x, y]=z^{i}
$$

for some $i$, depending on $\bar{x}, \bar{y}$, but not on the choice of the representatives $x$, $y$. Since $|z|=p$, we can view $i$ as an element of $G F(p)$. We set $\rho(\bar{x}, \bar{y})=i$. It is now easily verified that $\rho$ is a nondegenerate alternating bilinear form on $\bar{X}$. Since the symplectic group on a vector space is defined to be the group of linear transformations preserving a nondegenerate alternating bilinear form, this suggests the term "symplectic" for $p$-groups of this general form.

One of the principal procedures of local analysis is to investigate the action of an (elementary) abelian $p$-subgroup $A$ of a simple group $G$ on $A$-invariant subgroups $Y$ of $G$ for suitable primes $p$. The analysis depends critically on the generation (or nongeneration) of $Y$ by the centralizers of various subgroups of $A$. The following basic result is used repeatedly [84, Theorem 5.3.16 and 6.2.4].

PROPOSITION 7. If the noncyclic elementary abelian p-group $A$ acts on the $p^{\prime}$-group $Y, p$ a prime, then

$$
Y=\left\langle C_{Y}(B)|B \leqslant A,| A: B\right|\langle p\rangle .
$$

In particular,

$$
Y=\left\langle C_{Y}(a) \mid a \in A^{\#}\right\rangle .
$$

The so-called "Frattini argument", which is an immediate consequence of Sylow's theorem is also a fundamental tool of local analysis.

Proposition 8. If $Y \nabla X$ and $P \in S y l_{p}(Y)$ for some prime $p$, then

$$
X=Y N_{X}(P) \text {. }
$$

Finally some notational conventions. Throughout the paper, the letter $G$ will be reserved for arbitrary simple groups and more generally, for any group which is to be investigated by local analytic methods. For $x \in \mathscr{I}_{p}(G), p$ any prime, we shall for simplicity write $C_{x}$ for $C_{G}(x)$. However, we shall not use this contraction in any other connection.

We shall use the letter $G^{*}$ for $K$-groups, and usually the letter $X$ will denote an arbitrary finite group. We also adopt the bar convention for homomorphic images $\bar{X}$ of $X$-i.e., $\bar{Y}$ will denote the image in $\bar{X}$ of any subgroup or subset $Y$ of $X$.

5. The shape of the proof. With the aid of certain of the terms and ideas discussed in the preceding section, we can now describe the breakup of the analysis of simple groups. We first define some key general concepts that were first introduced by John Walter and me in [96] and [97]. An improved 
approach to some of these ideas was given by Bender in [20]. Again let $X$ be an arbitrary group.

Definition 1. $X$ is quasisimple if $X$ is perfect and $X / Z(X)$ is simple.

For example, if $X=S L(2, q), q$ odd, $q>3$, then $X$ is quasisimple, but not simple, $Z(X)$ being of order 2 and generated by $\left(\begin{array}{cc}-1 & -0 \\ 0 & -1\end{array}\right) .(S L(2,3)$ is solvable and hence is not perfect.)

If $X$ is quasisimple, one speaks of $X$ as being a covering group of $X / Z(X)$. At the present time (with the exception of at most one sporadic group), the possible covering groups of every simple $K$-group have been computed. It was shown by Schur [164] that a simple (and, more generally, perfect) group $X$ possesses a "universal" covering group $\hat{X}$ with the property that every covering group of $X$ is a homomorphic image of $\hat{X} . Z(\hat{X})$ is called the Schur multiplier of $X$. Thus the problem of determining the possible covering groups of $X$ reduces to computing the center of $\hat{X}$.

For example, $A_{n}$ has $Z_{2}$ as its Schur multiplier except for $n=6$ or 7 , in which cases the Schur multiplier is cyclic of order 6. Thus $A_{6}$ and $A_{7}$ have three nontrivial covering groups, whose centers have orders 2,3 , and 6 , respectively.

Definition 2. $X$ is semisimple if either $X$ is a central product of quasisimple groups or $X=1$.

If $X \neq 1$ is semisimple, it is easily shown that the quasisimple factors of $X$ are precisely the set of normal subgroups of $X$ which are minimal subject to being nonsolvable and, in particular, are uniquely determined by $X$. They are called the components of $X$. (One includes the trivial group in the definition solely for convenience.)

The following is easily proved.

Proposition 3. A finite group $X$ possesses a unique maximal normal semisimple subgroup. It is called the layer of $X$ and is denoted by $L(X)$.

It is immediate that $L(X)$ centralizes $\operatorname{Sol}(X)$ and hence $F(X)$.

The next concept, due to Bender, is fundamental and has its origin in some basic properties of solvable groups.

Proposition 4. If $X$ is a solvable group, then

$$
C_{X}(F(X)) \leqslant F(X) \text {. }
$$

In particular, $X$ is p-constrained for every prime $p$.

That the second assertion follows from the first is immediate from the definition of $p$-constraint and the fact that $F(X)=O_{p}(X)$ if $O_{p^{\prime}}(X)=1$.

It is this property of solvable groups which Bender generalized.

Definition 5. $F^{*}(X)=L(X) F(X)$ is called the generalized Fitting subgroup of $X$.

Justification for this terminology is given by the following general result of Bender (the special case in which $O_{p^{\prime}}(X)=1$ is proved in [96] and [97]).

Proposition 6. For any group $X, C_{X}\left(F^{*}(X)\right) \leqslant F^{*}(X)$. Moreover, if $F(X)$ $\leqslant Y \nabla X$ and $C_{X}(Y) \leqslant Y$, then $F^{*}(X) \leqslant Y$.

Thus $F^{*}(X)$ is the unique normal subgroup of $X$ which is minimal subject 
to containing $F(X)$ and to containing its own centralizer in $X$. For example, if $F^{*}=F^{*}(X)$ is simple, the proposition shows that $X \leqslant \operatorname{Aut}\left(F^{*}\right)$. More generally if $F^{*}$ is quasisimple, it yields that $X / Z\left(F^{*}\right) \leqslant \operatorname{Aut}\left(F^{*} / Z\left(F^{*}\right)\right)$. Likewise, as an immediate corollary of the proposition, we have

Corollary 7. For any group $X, C_{X}(F(X)) \leqslant F(X)$ if and only if $L(X)=$ 1. In particular, $X$ is $p$-constrained for any prime $p$ if and only if $L\left(X / O_{p^{\prime}}(X)\right)$ $=1$.

These concepts enable us to describe in group-theoretic terms the general structure of the centralizers of involutions in simple $K$-groups. Ree [162], Iwahori [128], and Burgoyne and Williamson [37], [38] have described these centralizers in the groups of Lie type of odd characteristic (the third pair of authors covering more generally the centralizers of "semisimple" elements in groups of Lie type of arbitrary characteristic). A detailed statement of their results will be given in Chapter IV. On the other hand, the general shape of such centralizers in groups of Lie type of characteristic 2 follows from a theorem of Borel and Tits [26] which gives the basic general structure of $p$-local subgroups in any group of Lie type of characteristic $p$.

We list here only the following general properties.

Proposition 8. If $G^{*}$ is of Lie type of characteristic $p, t^{*} \in \mathscr{G}\left(G^{*}\right)$, and we set $C^{*}=C_{G^{*}}\left(t^{*}\right)$, then we have

(i) If $p$ is odd, then $C^{*} / L\left(C^{*}\right)$ is solvable. In particular, either $L\left(C^{*}\right) \neq 1$ or $C^{*}$ is solvable.

(ii) If $p=2$, then $F^{*}\left(C^{*}\right)=O_{2}\left(C^{*}\right)$ (equivalently, $C^{*}$ is 2-constrained with trivial core).

It will be instructive for the reader to compute $C^{*}$ when $G^{*}=S L(m, q)$ in the following two cases:

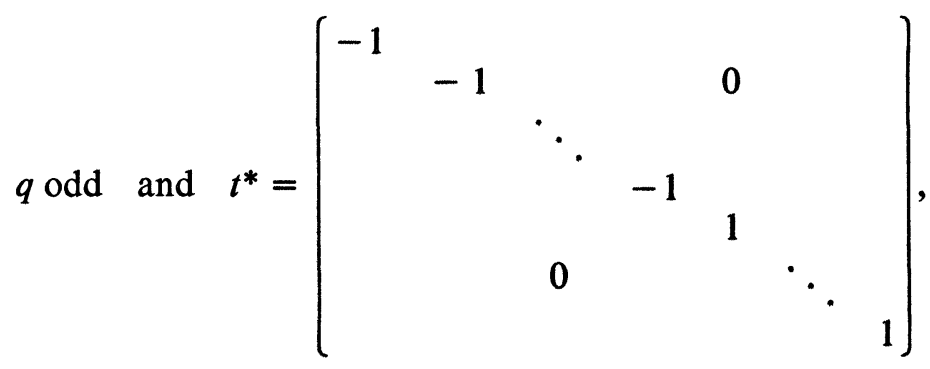

with $k$ entries $-1, k$ even. (1)

$q$ even and $t^{*}=\left(\begin{array}{ccccc}1 & & & & \\ 0 & 1 & & 0 & \\ \vdots & & \ddots & & \\ 0 & & & & \\ 1 & 0 & \vdots & 0 & 1\end{array}\right)$. 
In the first case, except for special values of $q$ and $k$,

$$
L\left(C^{*}\right)=S L(k, q) \times S L(m-k, q) .
$$

In the second case, $F^{*}\left(C^{*}\right)$ is a special 2-group of order $q^{2 n+1}$ with (elementary) center of order $q$.

The proposition shows a fundamental dichotomy in the structure of the centralizers of involutions in groups of Lie type of characteristic $p$ according as $p$ is odd or even. For odd $p$, their layers are always nontrivial (except in some small degenerate cases); while for $p=2$, these layers are always trivial and, in fact, their generalized Fitting subgroups are always 2-groups.

If $G^{*}=A_{n}$ and $t^{*} \in \mathscr{G}\left(G^{*}\right)$ is a product of $k$ disjoint transpositions, one easily checks that $L\left(C^{*}\right)$ is isomorphic to the alternating group on the remaining $n-2 k$ letters provided $n-2 k>5$; while in the contrary case, $L\left(C^{*}\right)=1$. In particular, if $n \geqslant 9$ and $t^{*}$ is a "short" involution, then $L\left(C^{*}\right) \neq 1$; while if $t^{*}$ is a "long" involution, then $L\left(C^{*}\right)=1$.

Likewise in the sporadic groups, the centralizer of some involutions have nontrivial layers and of others have trivial layers.

This dichotomy in the layers of centralizers of involutions is basic for the study of simple groups $G$. However, it is not $L\left(C_{G}(t)\right), t \in G(G)$, which is initially important, but rather $L\left(C_{G}(t) / O\left(C_{G}(t)\right)\right)$. In fact, as we shall see later, a central problem that must be resolved is the precise relationship between these two layers.

In view of the preceding discussion, it is therefore natural to divide all simple groups into two categories, as follows:

Definition 9, A group $X$ is said to be of component type if $L\left(C_{X}(t)\right) / O\left(C_{X}(t)\right) \neq 1$ for some involution $t$ of $X$; and is said to be of noncomponent type if $L\left(C_{X}(t)\right) / O\left(C_{X}(t)\right)=1$ for every involution $t$ of $X$.

We would next like to make more exact the term "small" simple group, which we have introduced here solely for its suggestive qualities. The more precise notion is that of "connectedness".

Definition 10. A group $X$ is said to be connected for the prime $p$ provided for any two noncyclic elementary abelian $p$-subgroups $A, B$ of $X$, there exists a sequence $A=A_{1}, A_{2}, \ldots, A_{n}=B$ of noncyclic elementary abelian $p$ subgroups $A_{i}$ of $X, 1 \leqslant i \leqslant n$, such that $A_{i}$ centralizes $A_{i+1}, 1 \leqslant i \leqslant n-1$.

If $p=2$, one says simply that $X$ is connected.

The term "connectedness" is a natural one, for if we form a graph $\Gamma$ whose vertices are the subgroups of $X$ isomorphic to $Z_{p} \times Z_{p}$ and connect two vertices of $\Gamma$ by an edge if and only if the corresponding subgroups centralize each other, then $X$ will be connected (for $p$ ) if and only if the resulting graph $\Gamma$ is connected in the usual sense.

An essential step in the classification of simple groups is the determination of all nonconnected groups (for the prime 2). Connectedness is a basic property of groups; in its presence, it is possible to carry out certain general lines of argument, which have powerful consequences for the structure of the cores of centralizers of involutions in simple groups. We shall give an illustration shortly.

Unfortunately, nonconnectedness is a difficult problem to treat directly because the condition is not inductive to sections. In practice, the problem 
has been treated in two parts, which together constitute two of the major achievements of simple group theory. The division is based upon the following facts. A simple group $G$ may be connected, but a Sylow 2-subgroup of $G$ may be nonconnected. Alternately, $G$ may be nonconnected, but have a connected Sylow 2-subgroup. Janko's second and third groups $J_{2}, J_{3}$ are examples of the first kind (as shown by Goldschmidt); while the groups $L_{2}\left(2^{n}\right)$ are examples of the second. Thus the problem of nonconnectedness has been divided into the following two parts:

A. Determine all nonconnected simple groups having a connected Sylow 2-subgroup.

B. Determine all simple groups having a nonconnected Sylow 2-subgroup.

We should next like to reformulate these problems in the terms in which they have actually been studied. A is directly connected with the notion of a proper 2-generated core, introduced in [95].

Definition 11. Let $G$ be a group, $p$ a prime, $P$ a Sylow $p$-subgroup of $G$ and $k$ a positive integer with $k \leqslant m_{p}(P)$. Define

$$
\Gamma_{P, k}(G)=\left\langle N_{G}(Q) \mid Q \leqslant P, m_{p}(Q) \geqslant k\right\rangle .
$$

$\Gamma_{P, k}(G)$ is called the $k$-generated $p$-core of $G$ and is determined up to conjugacy by $P$. If $p=2$, we speak of the $k$-generated core.

Proposition 12. Let $G$ be a group having a connected Sylow 2-subgroup. Then $G$ is disconnected if and only if the 2-generated core of $G$ is a proper subgroup.

Indeed, let $\Gamma_{0}$ be a connected component of the associated graph $\Gamma$ of $G$, let $T$ be a vertex of $\Gamma_{0}$ and $S$ a Sylow 2-subgroup of $G$ containing the four group $T$. Every element $g$ of $G$ induces by conjugation a permutation of $\Gamma$ and the image of $\Gamma_{0}$ under $g$ is the connected component of $\Gamma$ containing $T^{8}$. We denote it by $\Gamma_{0}^{g}$. Clearly $G$ is connected if and only if $G$ leaves $\Gamma_{0}$ invariant. Likewise $\Gamma_{0}^{g}=\Gamma_{0}$ if and only if $\Gamma_{0} \cap \Gamma_{0}^{g} \neq \varnothing$.

Set $H=\Gamma_{S, 2}(G)$. We shall argue that $H$ acts on $\Gamma_{0}$ and that every vertex of $\Gamma_{0}$ is a four subgroup of $H$, which will immediately yield that $H<G$ if and only if $\Gamma_{0}<\Gamma$ and hence if and only if $G$ is disconnected.

Note that as $S$ is assumed to be connected, its subgraph is connected, so the vertices of $\Gamma_{0}$ include every four subgroup of $S$. Now let $R \leqslant S$ with $m_{2}(R) \geqslant 2$ and let $U$ be any four subgroup of $R$. Then for $x \in N_{G}(R), U$ and $U^{x}(\leqslant R)$ are two four subgroups of $S$. Hence $U$ and $U^{x} \in \Gamma_{0}$. But as $U \in \Gamma_{0}, U^{x} \in \Gamma_{0}^{x}$, so $U^{x} \in \Gamma_{0} \cap \Gamma_{0}^{x}$, forcing $\Gamma_{0}=\Gamma_{0}^{x}$. Thus $x$ leaves $\Gamma_{0}$ invariant, so $N_{G}(R)$ leaves $\Gamma_{0}$ invariant. We conclude that $H=\Gamma_{S, 2}(G)=$ $\left\langle N_{G}(R) \mid R \leqslant S, m_{2}(R) \geqslant 2\right\rangle$ leaves $\Gamma_{0}$ invariant.

Now let $V$ be an arbitrary vertex of $\Gamma_{0}$. Let $T=T_{1}, T_{2}, \ldots, T_{n}=V$ be a chain of vertices of $\Gamma_{0}$ connecting $T$ to $V$. We have that $T=T_{1}<H$. If $T_{i} \leqslant H$, then $N_{G}\left(T_{i}\right) \leqslant H$ by definition of $H$, so $T_{i+1} \leqslant H$ as $T_{i+1}$ centralizes $T_{i}$. We thus conclude inductively that $V \leqslant H$. This establishes the proposition.

Aschbacher has determined all finite groups having a proper 2-generated core [6], generalizing Bender's fundamental classification of groups which 
possess a "strongly embedded" subgroup (equivalently which have a proper 1-generated core) [19]. These results will be discussed in Chapter IV.

On the other hand, a solution of B has been obtained as a corollary of a more general theorem classifying all groups of "sectional 2-rank at most 4". Because of this, groups of sectional 2-rank at most 4 are considered to be part of the category of "small" simple groups. To explain this concept, we introduce some general terminology.

Definition 13. Let $X$ be a $p$-group for some prime $p$. The rank of $X$, denoted by $m(X)$ (not to be confused with the (permutation) rank of a permutation group), is the maximum rank (in the ordinary sense) of an abelian subgroup of $X$. (An abelian $p$-group is always a direct product of cyclic groups, the number of factors being independent of the particular decomposition.) The normal rank of $X$, denoted by $n(X)$, is the maximum rank of an abelian normal subgroup of $X$. The sectional rank of $X$, denoted by $r(X)$ is the maximum rank of an abelian section of $X$.

Clearly $n(X) \leqslant m(X) \leqslant r(X)$. If $Y \leqslant X, m(Y) \leqslant m(X)$ and $r(Y) \leqslant$ $r(X)$, but the inequality need not hold for $n(Y)$. Moreover, if $S$ is a section of $X$, then $r(S) \leqslant r(X)$, but the inequality need not hold for either $m(X)$ or $n(X)$. Thus only the sectional rank is "inductive" to both subgroups and homomorphic images. It is for this reason that the concept of sectional rank is important in the study of simple groups.

There are two major results about $p$-groups of very low rank or normal rank (cf. [84, Chapter 5]).

Proposition 14. If $X$ is a p-group, we have

(i) If $m(X)=1$, then either $X$ is cyclic or $p=2$ and $X$ is quaternion.

(ii) If $n(X)=1$ and $m(X)>1$, then $p=2$ and $X$ is either quaternion, dihedral of order at least 16, or quasi-dihedral.

In particular, $r(X) \leqslant 2$ in either case.

Results of Blackburn [24], [25] and MacWilliams [142] give deeper properties of $p$-groups of normal rank 2 .

THEOREM 15. If $X$ is a p-group with $n(X)=2$, then we have

(i) If $p$ is odd, then $m(X)=2$.

(ii) If $p=2$, then $r(X) \leqslant 4$.

We extend these notions of rank to arbitrary groups, as follows.

DEFINITION 16. For any group $X$, the $p$-rank, normal $p$-rank, and sectional $p$-rank, denoted by $m_{p}(X), n_{p}(X), r_{p}(X)$, respectively, is defined to be $m(P)$, $n(P), r(P)$, respectively, for any $P \in S y l_{p}(X)$.

One easily proves

Proposition 17. If $X$ is a 2-group with $n(X) \geqslant 3$, then $X$ is connected.

Combined with Theorem 15 , this yields

COROLlaRY 18. If $X$ is a 2-group with $r(X) \geqslant 5$, then $X$ is connected.

Thus a simple group of sectional 2-rank at least 5 always has a connected Sylow 2-subgroup. The advantage of studying groups $G$ of sectional 2-rank at most 4 (instead of the more restricted problem of groups with a nonconnected 
Sylow 2-subgroup) lies in the fact that this condition is satisfied by every section of $G$ and so one can proceed inductively to try to classify all such groups. This has indeed been carried out by Harada and me [89]. Our proof will be discussed in Chapter V, along with some of the prior classification theorems concerning groups of low 2-rank, which are needed for the argument.

We conclude this discussion with the statement of a particular case of a theorem of John Walter and me, [95], [85], which will give some indication of the force of connectedness. (Its proof utilizes several general local grouptheoretic techniques, which will be discussed later.)

THEOREM 19. Let $G$ be a group of noncomponent type of 2-rank at least 3 with $O(G)=1$. If $G$ is connected or if $G$ has a connected Sylow 2-subgroup, then $F^{*}(H)$ is a 2-group for any 2-local subgroup $H$ of $G$ (equivalently, $O(H)=1$ and $H$ is 2-constrained). In particular, this holds for the centralizer of every involution of $G$.

There are also notions of "smallness" for connected simple groups of noncomponent type. Such groups satisfy the conclusion of Theorem 19. To put this in its proper context, it will be helpful first to say a few words about the groups of Lie type.

It was Chevalley [41] who gave the first systematic treatment of the finite analogues of the complex Lie groups. Because of this, we denote by $\operatorname{Chev}(p)$ the set of groups of Lie type defined over a field of characteristic $p$. Their structure is well understood in many aspects and will be described in more detail in Chapter II.

Let $X \in \operatorname{Chev}(p)$ for some prime $p$. If $P \in S y l_{p}(X)$, then $B=N_{X}(P)$ is called a Borel subgroup of $X$. By Sylow's theorem, all Borel subgroups of $X$ are conjugate. By the Schur-Zassenhaus theorem [84, Theorem 6.2.1] $B$ splits over $P$-i.e., $B=C P$, where $C$ is a subgroup of $B$ such that $C \cap P=1$. $C$ is called a Cartan subgroup of $X$. All Cartan subgroups of $X$ are conjugate. It is known that $C$ is always abelian and except possibly when $p=2$ and $X$ is defined over the prime field $G F(2)$ (also in the case $X=L_{2}(3)$ ), $C$ is nontrivial. Any proper subgroup of $X$ containing $B$ is called a parabolic subgroup of $X$. It is known that every parabolic subgroup $J$ of $X$ is $p$ constrained with trivial $p^{\prime}$-core; that is, $F^{*}(J)$ is a $p$-group for every parabolic subgroup $J$ of $X$.

The Borel-Tits theorem, mentioned earlier, gives a purely group-theoretic characterization of the maximal parabolics.

THeOREM 20. If $X \in C h e v(p)$, then every maximal p-local subgroup of $X$ is a parabolic subgroup.

Thus $F^{*}(H)$ is a $p$-group for every maximal $p$-local subgroup $H$ of $X$. Combining this with an easy group-theoretic argument, one can obtain the following extension of the theorem.

THEOREM 21. If $X \in C$ Chev $(p)$, then $F^{*}(H)$ is a p-group for every $p$-local subgroup $H$ of $X$.

In view of the theorem, it is natural to make the following definition. 
Definition 22. A group $X$ is said to be of characteristic $p$ type if $F^{*}(H)$ is a $p$-group for every $p$-local subgroup $H$ of $X$.

Thus Theorem 19 asserts that a connected simple group of noncomponent type and 2-rank at least 3 must be of characteristic 2 type.

In the study of simple groups of component type, the role of centralizers of involutions is much more dominant than in groups of noncomponent type (and, in particular, groups of characteristic 2 type). This is because the "prototypes" of such groups are the groups $G^{*} \in \operatorname{Chev}(p), p$ odd, and in such a group $G^{*}$, involutions correspond to "semisimple" elements (in the Lie sense), especially those involutions lying in a Cartan subgroup of $G^{*}$.

As we have pointed out in the dedication, Brauer demonstrated this domination explicitly in the groups $L_{3}(q), U_{3}(q), q$ odd (and later, jointly with Suzuki and Wall in the groups $L_{2}(q)$ [33]). For a simple group $G$ which internally resembles a group $G^{*}$ of Lie type of odd characteristic of higher dimension, general methods (not requiring character theory) have been developed for passing from the centralizers of involutions of $G$ to either the so-called $(B, N)$-pair axioms of Tits or the Steinberg relations (to be discussed later), which in turn enable one to prove that $G$ is isomorphic to $G^{*}$.

It is also possible to determine $G$ from the centralizers of its involutions when $G$ internally resembles a group $G^{*} \in \operatorname{Chev}(2)$. In particular, this has been done by Suzuki for most of the classical groups [193], [194], [195]. Thus, theoretically at least, if one could show that the centralizer of an involution in a simple group $G$ of characteristic 2 type resembled that in some simple $K$-group $G^{*}$, one should then be able to show that $G$ and $G^{*}$ are isomorphic. However, in most situations in such a group $G$, it can be very difficult to force $O_{2}\left(C_{G}(t)\right), t \in g(G)$, to resemble $O_{2}\left(C_{G^{*}}\left(t^{*}\right)\right)$ for some simple $K$-group $G^{*}$ and some $t^{*} \in \mathscr{G}\left(G^{*}\right)$.

Rather, the crucial objects of investigation in simple groups $G$ of charactersitic 2 type are the centralizers of elements $x$ of odd prime order $p$ which lie in a 2-local subgroup of $G$. In trying to show that $G$ resembles internally a simple group $G^{*} \in \operatorname{Chev}(2)$, the ultimate aim is to choose $p$ to be a prime which divides the order of the Cartan subgroup of the corresponding group $G^{*}$ and $x$ to correspond to an element of such a Cartan subgroup. Once this is achieved, $x$ will again correspond to a "semisimple" element of $G^{*}$ and the proof that $G$ and $G^{*}$ are isomorphic follows the same general pattern as the corresponding argument for centralizers of involutions in groups of component type. (In those cases in which $G^{*}$ has a trivial Cartan subgroup -i.e., when $G^{*}$ is Chevalley group defined over $G F(2)$-one works with the prime $p=3$.)

Just as special methods are required for studying centralizers of involutions in groups of low 2-rank, the same is true when analyzing centralizers of elements of odd prime order in groups of characteristic 2 type which have "small" $p$-rank for all odd primes $p$. However, in this case the notion of smallness is a "relativized" one, which we proceed to define.

Definition 23. Let $X$ be a group of characteristic 2 type. For any odd prime $p$, the 2-local $p$-rank of $X$, denoted by $m_{2, p}(X)$, is the maximum of $m_{p}(H)$, taken over all 2-local subgroups $H$ of $X$. Furthermore, we set 


$$
e(X)=\max \left\{m_{2, p}(X) \mid p \text { any odd prime }\right\} .
$$

We call $e(X)$ the odd 2-local rank of $X$.

A classical theorem of Frobenius asserts that if $e(X)=0$ (equivalently, every 2-local subgroup of $X$ is a 2-group), then $X$ has normal 2-complement. Thus $e(G) \geqslant 1$ in any simple group $G$ of characteristic 2 type.

"Smallness" for odd primes in simple groups $G$ of characteristic 2 type means precisely that $e(G)=1$ or 2 . Correspondingly we call $G$ a thin or quasithin group. Centralizers of elements of odd prime order dominate the analysis when $e(G)>3$. In contrast, the analysis of thin and quasithin groups focuses directly on the structure of the maximal 2-local subgroups of $G$. (On the other hand, there are strong similarities in later stages of the arguments in both the $e(G) \geqslant 3$ and $e(G) \leqslant 2$ problems.)

There is yet a second notion of "smallness" connected with groups of characteristic 2 type which has no counterpart in the study of groups of component type. In Thompson's classification of $N$-groups, the proof divided into four major subcases:
(A) $e(G) \geqslant 3$;
(B) $e(G)=2$;
(C) $e(G)=1$;
(D) $\mathrm{O}_{2}\left(C_{G}(t)\right)$ is of symplectic type for some $t \in G(G)$.

This last possibility (D) cut across all three of the preceding cases; in its presence, certain general lines of argument collapse. To handle it, Thompson was forced to produce a completely independent argument.

Since the analysis of arbitrary simple groups $G$ of characteristic 2 type can be viewed in many ways as a direct generalization of Thompson's $\mathrm{N}$-group proof (a further measure of depth of his vision), one can expect the $\mathrm{O}_{2}$ symplectic type case (i.e. case (D)) to remain an important special problem. Moreover, it corresponds to a "real life" situation in many of the groups $G^{*}$ of Lie type defined over the prime field $G F(2)$. For example, if $G^{*}=$ $S L(m, 2)$ and one takes $t^{*}$ as in (2) above (p. ), then

$$
F^{*}\left(C^{*}\right)=O_{2}\left(C^{*}\right) \cong\left(D_{8}\right)^{m-2} \text { is extra-special. }
$$

This will explain the following terminology.

Definition 24. A group $X$ is said to be of $G F(2)$-type if for some involution $t$ of $X, F^{*}\left(C_{X}(t)\right)$ is a 2-group of symplectic type.

Although our primary interest is in the case in which $X$ is of characteristic 2 type, note that the definition requires 2-constraint only for $C_{X}(t)$ itself.

Remarkably, more than half of the sporadic groups are of $G F(2)$-type. It is for this reason and partly for other aspects of their internal structure that sporadic groups are often considered to be pathologies of groups of Lie type over $G F(2)$.

6. The four phases of the classification. Summarizing the preceding discussion, we see that the classification of simple groups divides into the classification of simple groups in each of the following four categories:

(A) Nonconnected simple groups,

(B) Connected simple groups of component type,

(C) Small simple groups of characteristic 2 type,

(D) Simple groups of characteristic 2 type of large odd 2-local rank. 
Specifically, (A) refers to the determination of all simple groups having either a proper 2-generated core or sectional 2-rank at most 4; (B) to the determination of all connected simple groups of component type of 2-rank at least 3 ; (C) to the determination of all thin and quasithin simple groups of characteristic 2 type and of all simple groups of type $G F(2)$; and (D) to the determination of all simple groups of characteristic 2 type with $e(G) \geqslant 3$ and not of $G F(2)$-type.

In Chapters V-VIII, we shall outline in succession the results so far established concerning simple groups within each of the four categories (A), (B), (C), (D). However, to make that discussion meaningful, it is first necessary, on the one hand, to describe the known simple groups in some reasonable fashion; and, on the other, to explain the major tools of local analysis, at least in general terms. This we shall try to do in Chapters II, III, and IV.

Finally in Chapter IX, we shall present a precise list of the specific problems remaining to determine all simple groups. ${ }^{9}$

In writing this article, it has been my hope to convince the reader that the classification of the finite simple groups can be expected within the next few years. However, even after the classification, certain questions about existence and, uniqueness will very likely remain, related to a single family of groups of Lie type and to a few specific sporadic simple groups. These unanswered questions will, of course, require resolution before the classification can be considered to be "complete". This point is discussed more fully in §III.2.

\footnotetext{
${ }^{9}$ In referring to statements of results within the paper we use the following notational convention: Theorem x.y.z will refer to Theorem $z$ of $\$ y$ of Chapter $x$. If the reference is being made within Chapter $\mathrm{x}$, we suppress the $\mathrm{x}$ and write Theorem $\mathrm{y} \cdot \mathrm{z}$. Moreover, within $\S \mathrm{y}$ of Chapter $\mathbf{x}$, we refer to the given result as Theorem $\mathrm{z}$. The same conventions apply to references to definitions and to sections.
} 
Much of the excitement generated by the developments in simple group theory over the past 20 years can be directly attributed to the discovery of over 20 new sporadic groups, the first of these, Janko's group of order 175,560 , constructed in 1965 , coming almost exactly 100 years after the five groups of Mathieu. The existence of these strange objects, discovered at a rate of about one per year, revealed the richness of the subject and lent an air of mystery to the nature of simple groups. We wish to give a brief account of the 26 presently known sporadic groups. ${ }^{10}$ At the same time we shall describe the groups of Lie type, including those of Suzuki and Ree. These together with the familiar alternating groups comprise the set of currently known simple groups.

It is essential for the reader to distinguish between the notions of discovery and classification. The search for new simple groups has been carried out to a large extent completely independently of the classification problem. This phenomenon can be compared to elementary particle theory, in which one must scan a large horizon with the aid of one's intuition and theoretical knowledge in the hope of distinguishing a new particle. If the automorphism group of a certain integral lattice has yielded a new group, then look for other lattices which may have "large" automorphism groups. If Janko's second group has turned out to be a primitive rank 3 permutation group, then conduct a more general investigation of such permutation groups. If simple groups are intimately connected with the centralizers of their involutions, choose a likely candidate and investigate whether there can be a new simple group with such a centralizer of an involution; etc. Any plausible direction is worth considering; just keep in mind that the probability of success is very low.

In contrast, general classification problems are all-inclusive, the goal of the analysis being the systematic determination of all simple groups with a given property. In particular, the argument can be successful only if it "uncovers" every sporadic group having the specified property.

1. The groups of Lie type. There are four families of simple complex Lie groups: $A_{n}(\mathbf{C}), B_{n}(\mathbf{C}), C_{n}(\mathbf{C}), D_{n}(\mathbf{C})$, corresponding, respectively, to the linear groups $S L(n+1, \mathrm{C})$, the orthogonal groups $S O(2 n+1, \mathrm{C})$, the symplectic groups $\operatorname{Sp}(2 n, \mathbf{C})$, and the orthogonal groups $S O(2 n, \mathrm{C})$. In addition, there are five exceptional Lie groups $G_{2}(\mathbf{C}), F_{4}(\mathbf{C}), E_{6}(\mathbf{C}), E_{7}(\mathbf{C}), E_{8}(\mathbf{C})$. As Elie Cartan showed in the last century, these arise as automorphism groups of the corresponding simple Lie algebras. Finite analogues of many of these groups were known long before Chevalley-certainly the analogues of the classical

\footnotetext{
${ }^{10}$ At this writing, the question of the existence of Janko's fourth group and the Fischer monster is unresolved, but they are counted among the 26 sporadic groups.
} 
groups; but also Dickson had constructed the analogue of $G_{2}(C)$ and $E_{6}(C)$ early in the century. However, Chevalley gave the first systematic treatment of the whole subject [41].

He first proved that every complex Lie algebra $\mathcal{L}$ has an integral basis-i.e., with respect to this basis, the coefficients of the Lie product of any two elements of $\mathcal{L}$ are all integers. (It was known well before Chevalley that a basis existed with respect to which the coefficients were rational.) For any field $K$ there is a natural ring homomorphism of the ordinary integers $\mathbf{Z}$ into the prime field of $K$. Thus, with the aid of this integral basis, Chevalley was able to view the exponentiation formulas which describe the automorphisms of $\mathcal{L}$ that generate the corresponding Lie group as defined relative to the given field $K$ rather than to the complex numbers $\mathbf{C}$. By this method, he was led to define, for each choice of $\mathcal{E}$ and $K$, a specific group by means of an appropriate set of generators (the so-called "adjoint" groups associated with $\mathcal{L}$ and $K)$. In particular, these groups are finite when $K$ is finite.

Thus for each choice of the Galois field $K=G F(q)$ with $q$ elements, $q$ a prime power, there exist finite groups $A_{n}(q), B_{n}(q), C_{n}(q), D_{n}(q), G_{2}(q), F_{4}(q)$, $E_{6}(q), E_{7}(q), E_{8}(q)$, known as the (finite) Chevalley groups.

The complex unitary group $G U(n, \mathrm{C})$ is an important subgroup of the general linear group. It consists of all nonsingular complex matrices $U \in$ $A_{n}(\mathrm{C})$ such that

$$
U=\left((\bar{U})^{t}\right)^{-1}
$$

where $(\bar{U})^{t}$ denotes the transpose of the complex conjugate $\bar{U}$ of $U$. One can describe the unitary group more algebraically as follows. For each element $X \in A_{n}(\mathbf{C})$, set

$$
\alpha(X)=\left((\bar{X})^{t}\right)^{-1}
$$

Then $\alpha$ is an automorphism of $A_{n}(\mathrm{C})$ of period 2; and the unitary group is precisely the set of elements of $A_{n}(\mathrm{C})$ left fixed by $\alpha$. In the Lie notation, the unitary group is denoted by ${ }^{2} A_{n}(\mathbf{R})$. It is the compact real form of the linear group.

Conjugation of the orthogonal group $S O(2 n, \mathrm{C})$, by an orthogonal transformation of determinant -1 and order 2 induces an outer automorphism of period 2. In the Lie theory, such an automorphism corresponds to a so-called "graph" automorphism of $D_{n}(\mathrm{C})$. Moreover, the exceptional group $E_{6}(\mathrm{C})$ also has a graph automorphism of period 2. Hence if we let $\alpha$ again denote the product of complex conjugation with the corresponding graph automorphism, we can similarly define the compact real form ${ }^{2} D_{n}(\mathbf{R})$ of the orthogonal group $D_{n}(\mathbf{C})$ and the compact real form ${ }^{2} E_{6}(\mathbf{R})$ of $E_{6}(\mathbf{C})$.

Soon after Chevalley, Steinberg [183] showed that Chevalley's ideas could be carried over with no essential change to establish finite analogues ${ }^{2} A_{n}(q)$, ${ }^{2} D_{n}(q),{ }^{2} E_{6}(q)$ of the corresponding compact real Lie groups, where in place of complex conjugation (which is an automorphism of $\mathbf{C}$ of period 2), one uses the Frobenius automorphism $\phi$ of the field $G F\left(q^{2}\right)$ of period 2 defined by 


$$
\phi(x)=x^{q} \text { for } x \in G F(q) .
$$

In particular, one can define in this way the finite general unitary group $G U(m, q)$ and its subgroup $S U(m, q)$ of elements of determinant 1 . In the Lie notation, one has

$$
{ }^{2} A_{n}(q)=S U(n+1, q) /(\bmod \text { scalars })=U_{n+1}(q)=\operatorname{PSU}(n+1, q) .
$$

A word about the orthogonal groups. Over $\mathbf{C}$, all nondegenerate symmetric bilinear forms are equivalent and so for each $m$, there is a uniquely determined group $S O(m, \mathrm{C})$. However, over $G F(q)$, there are always two such inequivalent forms, which determine two families of orthogonal groups. The corresponding groups are distinct if $m$ is even and are denoted by $\mathrm{SO}^{+}(m, q)$ and $\mathrm{SO}^{-}(m, q)$, respectively. On the other hand, if $m$ is odd, they are the same groups and are denoted by $S O(m, q)$ (sometimes, for convenience, by $\left.\mathrm{SO}^{+}(m, q)\right)$. These groups are, in general, nonsimple. One denotes their derived groups by $\Omega_{m}^{+}(q)$ and $\Omega_{m}^{-}(q)$, respectively, and one has

$$
\left|S O^{ \pm}(m, q): \Omega_{m}^{ \pm}(q)\right|=1,2 \text {, or } 4 \text { and }\left|Z\left(S O^{ \pm}(m, q)\right)\right|=1 \text { or } 2 \text {. }
$$

Finally, the factor groups $\Omega_{m}^{ \pm}(q) / Z\left(\Omega_{m}^{ \pm}(q)\right)$ are denoted correspondingly by $P \Omega_{m}^{+}(q)$ and $P \Omega_{m}^{-}(q)$. For $m$ odd, one also writes simply $\Omega_{m}(q)$ and $P \Omega_{m}(q)$. In the Lie notation, one has the following isomorphisms.

$$
P \Omega_{2 n+1}(q)=B_{n}(q), \quad P \Omega_{2 n}^{+}(q)=D_{n}(q) \text { and } P \Omega_{2 n}^{-}(q)={ }^{2} D_{n}(q) \text {. }
$$

The notation $O_{m}^{ \pm}(q)$ for $S O^{ \pm}(m, q) / Z\left(S O^{ \pm}(m, q)\right)$ is also standard (likewise $O_{m}(q)$ for $m$ odd).

The classical group $D_{4}(C)$ is the only complex Lie group having a graph automorphism of period 3 (the fixed subgroup of which is the Lie group $\left.G_{2}(C)\right)$. Since $\mathbf{C}$ has no automorphism of period 3, it is not possible to use this automorphism to construct a twisted group as in the other cases. On the other hand, in the field $G F\left(q^{3}\right)$, the Frobenius map $\phi(x)=x^{q}$ for $x \in G F\left(q^{3}\right)$ is an automorphism of period 3 and taking its product with the graph automorphism of period 3, we can construct a twisted group just as before, called the triality twisted $D_{4}(q)$ and denoted by ${ }^{3} D_{4}(q)$.

These twisted groups are referred to as the Steinberg variations of the Chevalley groups.

Although Suzuki did not discover his family of simple groups $S z\left(2^{n}\right), n$ odd, $n>1$, from the Lie theory, but rather in the process of determining all simple groups in which the centralizer of every involution is a 2-group [188], [189], Ree observed that they could equally well be constructed by a suitable twisting of the orthogonal groups $B_{2}\left(2^{n}\right)$. Indeed, it was known earlier that because of certain degeneracies in the multiplication coefficients, the three families $B_{2}\left(2^{n}\right), G_{2}\left(3^{n}\right)$, and $F_{4}\left(2^{n}\right), n$ odd, possess an "extra" automorphism, not accounted for by the general theory. From Suzuki's defining relations for his group, Ree was led to play a variation of the Steinberg twisting game with each of these automorphisms [160], [161] taking as $\alpha$ the product of the "extra" automorphism together with a suitable automorphism of $G F\left(p^{n}\right)$ ( $p=2$ or 3), which exists only for odd $n$. He obtained in this way three families of groups, the first of which was just the groups $S z\left(2^{n}\right)$. These families are denoted by ${ }^{2} B_{2}\left(2^{n}\right),{ }^{2} G_{2}\left(3^{n}\right)$, and ${ }^{2} F_{4}\left(2^{n}\right), n$ odd. 
The (finite) groups of Lie type consist of the Chevalley groups together with the Steinberg and Suzuki-Ree variations together with all their covering groups. As we have stipulated, we denote by $\operatorname{Chev}(p)$ the subset of these which are defined over $G F\left(p^{m}\right)$ for a given prime $p$. The combined analysis of Chevalley, Steinberg, and Suzuki-Ree (plus Tits [212], who proved the simplicity of the group $\left.\left({ }^{2} F_{4}(2)\right)^{\prime}\right)$ yields the following results.

THEOREM 1. If $G^{*}$ is a group of Lie type with $Z\left(G^{*}\right)=1$, then either $G^{*}$ is simple or one of the following holds:

(i) $G^{*}=A_{1}(2), A_{1}(3),{ }^{2} A_{2}(2)$, or ${ }^{2} B_{2}(2)$, and $G^{*}$ is solvable;

(ii) $G^{*}=B_{2}(2) \cong \Sigma_{6}$;

(iii) $G^{*}=G_{2}(2),\left|G^{*}:\left(G^{*}\right)^{\prime}\right|=2$, and $\left(G^{*}\right)^{\prime} \cong{ }^{2} A_{2}(3)$;

(iv) $G^{*}={ }^{2} G_{2}(3),\left|G^{*}:\left(G^{*}\right)^{\prime}\right|=3$, and $\left(G^{*}\right)^{\prime} \cong A_{1}(8)$; or

(v) $G^{*}={ }^{2} F_{4}(2),\left|G^{*}:\left(G^{*}\right)^{\prime}\right|=2$, and $\left(G^{*}\right)^{\prime}$ is simple.

Implicit in this statement is the exclusion of the families $B_{1}, C_{1}, C_{2}, D_{2}, D_{3}$, and ${ }^{2} D_{3}$ because of the following isomorphisms $\left(D_{1}\right.$ does not exist in the Lie theory):

$$
\begin{aligned}
& C_{1} \cong B_{1} \cong A_{1}, \quad C_{2} \cong B_{2}, \\
& D_{2} \cong A_{1} \times A_{1}, \quad D_{3} \cong A_{3}, \quad{ }^{2} D_{2}(q) \cong A_{1}\left(q^{2}\right), \quad{ }^{2} D_{3} \cong{ }^{2} A_{3} .
\end{aligned}
$$

Furthermore, one has

$$
B_{n}(q) \cong C_{n}(q) \text { for } q=2^{m} .
$$

There are some other isomorphisms among the simple groups of Lie type of low order; namely,

$$
A_{1}(4) \cong A_{1}(5), \quad A_{1}(7) \cong A_{2}(2) \quad \text { and } \quad B_{2}(3) \cong{ }^{2} A_{3}(2) .
$$

In addition, three groups of Lie type are isomorphic to alternating groups:

$$
A_{1}(4) \cong A_{5}, \quad A_{1}(9) \cong A_{6}, \text { and } A_{3}(2) \cong A_{8}
$$

The groups of Lie type have a uniform description in terms of their so-called Bruhat decomposition. This was worked out by Chevalley and extended by Steinberg to the twisted groups. We need a preliminary definition.

Definition 2. A group $W$ will be said to be a Coxeter group or a group generated by reflections provided:

(1) $W$ is generated by distinct involutions $w_{i}, 1 \leqslant i \leqslant m$; and

(2) If $w_{i} w_{j}$ has order $k_{i j}$, then the relations

$$
\left(w_{i} w_{j}\right)^{k_{i j}}=1, \quad 1 \leqslant i, j \leqslant m,
$$

constitute a complete set of defining relations for $W$.

For example, the symmetric group $\Sigma_{m+1}$ is a Coxeter group with respect to the transpositions $w_{i}=(i, i+1), 1 \leqslant i \leqslant m$.

The integer $m$ is called the rank of $W$. Note that if $m=1$, then $|W|=2$, while if $m=2$, then $W$ is a dihedral group of order $2 k_{12}$. Moreover, the involutions $w_{i}, 1 \leqslant i \leqslant m$, are called a defining set for $W$.

Theorem 3. Let $G^{*} \in \operatorname{Chev}(p)$, let $B$ be a Borel subgroup of $G^{*}(B=$ $\left.N_{G^{*}}(P), P \in S y l_{p}\left(G^{*}\right)\right)$, and let $H \leqslant B$ be a Cartan subgroup of $G^{*}(H$ is a 
complement to $P$ in $B$ ). Then $G^{*}$ possesses a subgroup $N$ with the following properties:

(i) $B \cap N=H \triangleleft N$ and $W=N / H$ is a Coxeter group;

(ii) $G^{*}=B N B$

(iii) Let $w_{i}, 1 \leqslant i \leqslant m$, be a defining set for $W$ and let $v_{i}$ be a representative of $w_{i}$ in $N$. Then for each $v \in N$ and every $i, 1 \leqslant i \leqslant m$, we have

$$
B v B v_{i} B \leqslant(B v B) \cup\left(B v v_{i} B\right)
$$

and

(iv) $B^{v_{i}} \neq B, 1 \leqslant i \leqslant m$.

The integer $m$ is called the Lie rank of $G^{*}$ and $W$ is called the Weyl group of $G^{*}$. The double coset multiplication formulas of (iii) give very strong restrictions on the structure of $G^{*}$. In fact, it is not too difficult to prove that (ii) is a consequence of (iii) and the fact that $G^{*}=\langle B, N\rangle$.

As an immediate corollary of the theorem, one has

Corollary 4. If $G^{*} \in \operatorname{Chev}(p)$ is of Lie rank 1 with $Z\left(G^{*}\right)=1$, then $G^{*}$ is a doubly transitive group in which the stabilizer of a point is a Borel subgroup of $G^{*}$.

This doubly transitive permutation representation will be described in more detail in the next chapter, where we shall also take a closer look at the defining relations for the groups of Lie type.

2. The Mathieu groups. In the course of searching for highly transitive permutation groups, Mathieu discovered, about 1860, two such quintuply transitive groups, of respective degrees 12 and 24 and order $8 \cdot 9 \cdot 10 \cdot 11 \cdot 12$ and $3 \cdot 16 \cdot 20 \cdot 21 \cdot 22 \cdot 23 \cdot 24$ [227], [228], [229]. They are denoted by $M_{12}$ and $M_{24}$; likewise $M_{11}$ denotes the one-point stabilizer in $M_{12}, M_{23}$ the one-point stabilizer in $M_{24}$, and $M_{22}$ the one-point stabilizer in $M_{23}$. (Thus $\left|M_{11}\right|=8 \cdot 9 \cdot 10 \cdot 11,\left|M_{23}\right|=3 \cdot 16 \cdot 20 \cdot 21 \cdot 22 \cdot 23$, and $\left|M_{22}\right|=3 \cdot 16 \cdot 20$ $\cdot 21 \cdot 22$.) Each of these five groups is simple and together they represent the first five sporadic simple groups. Remarkably, apart from the alternating and symmetric groups themselves, they include the only known quadruply and quintuply transitive permutation groups. Equally surprising is the fact that it took over a hundred years for the sixth sporadic simple group to be discovered.

Since their discovery, many descriptions of the Mathieu groups have been given. We shall present three: the first as permutation groups, the second as groups of transformations of projective lines and the third as groups of automorphisms of so-called Steiner triple systems (cf. [39, Chapter XIII]).

Let $A, B, C$ be the following three permutations:

$$
\begin{aligned}
& A=(1,2,3,4,5,6,7,8,9,10,11), \\
& B=(5,6,4,10)(11,8,3,7), \\
& C=(1,12)(2,11)(3,6)(4,8)(5,9)(7,10) .
\end{aligned}
$$

Theorem 1. $M_{11}=\langle A, B\rangle$ and $M_{12}=\langle A, B, C\rangle$.

Let $D, E, F$ denote the following three permutations. 


$$
\begin{gathered}
D=(1,2,3,4,5,6,7,8,9,10,11,12,13,14,15,16,17,18,19,20,21,22,23), \\
E=(3,17,10,7,9)(5,4,13,14,19)(11,12,23,8,18)(21,16,15,20,22), \\
F=(1,24)(2,23)(3,12)(4,16)(5,18)(6,10)(7,20)(8,14)(9,21) \\
(11,17)(13,22)(19,15) .(2)
\end{gathered}
$$

THEOREM 2. $M_{23}=\langle D, E\rangle$ and $M_{24}=\langle D, E, F\rangle$.

Finally let $G, H, I$ denote the following three permutations.

$$
\begin{aligned}
G & =(1,2,3,4,5,6,7,8,9,10,11)(12,13,14,15,16,17,18,19,20,21,22), \\
H & =(1,4,5,9,3)(2,8,10,7,6)(12,15,16,20,14)(13,19,21,18,17), \\
I & =(11,22)(1,21)(2,10,8,6)(12,14,16,20)(4,17,3,13)(5,19,9,18) .
\end{aligned}
$$

THEOREM 3. $M_{22}=\langle G, H, I\rangle$.

Consider next the projective line $\mathscr{P}_{1}(q)$ coordinatized by the finite field $G F(q)$, with the usual convention that $a / 0=\infty$ if $a \neq 0, a \in G F(q)$. There are $q+1$ points on $\mathscr{P}_{1}(q)$, each represented by $a / b$ with $a, b \in G F(q)$ and $a$ or $b \neq 0$. The projective linear group $\operatorname{PGL}(2, q)$ or "fractional" linear group, as it is often called, can be viewed as a permutation group of these $q+1$ points. As is easily checked, it is triply transitive on this set.

The Mathieu groups $M_{12}$ and $M_{24}$ can be defined as extensions of the groups $L_{2}(11)$ and $L_{2}(23)$ (of index 2 in $P G L(2,11), P G L(2,23)$, respectively) by certain polynomial transformations of $\mathscr{P}_{1}(11)$ and $\mathscr{P}_{1}(23)$, respectively.

THEOREM 4. If $f$ denotes the transformation of $\mathcal{P}_{1}(11)$ given by

$$
f: x^{\prime}=4 x^{2}-3 x^{7},
$$

then $M_{12}=\left\langle L_{2}(11), f\right\rangle$.

THEOREM 5. If $f$ denotes the transformation of $\mathscr{P}_{1}(23)$ given by

$$
f: x^{\prime}=-3 x^{15}+4 x^{4},
$$

then $M_{24}=\left\langle L_{2}(23), f\right\rangle$.

Using a nonpolynomial transformation of $\mathscr{P}_{1}(23)$, Conway has given the following neat description of $M_{24}$ [43].

THEOREM 6. Let $f$ denote the transformation of $\mathcal{P}_{1}(23)$ given by

$$
f: x^{\prime}=9^{8} x^{3},
$$

where $\varepsilon=-1$ if $x$ is a square in $G F(23)$ and $\varepsilon=+1$ if $x$ is a nonsquare in $G F(23)$. Then $M_{24}=\left\langle L_{2}(23), f\right\rangle$.

Finally we consider Steiner systems for the Mathieu groups.

Definition 7. Let $\Omega$ be a set of $n$ elements. A Steiner triple system $S(k, m, n)$ on $\Omega$ is defined to be a set of $\left(\begin{array}{c}n \\ k\end{array}\right) /\left(\begin{array}{c}m \\ k\end{array}\right)$ subsets of $\Omega$, each of size $m$, with the property that every set of $k$ elements of $\Omega$ lies in one and only one of these subsets.

By definition, the automorphism group of $S(k, m, n)$ is the subgroup of 
$\Sigma(\Omega)$ whose elements transform the $m$-tuples of the Steiner system into themselves.

If one considers the subset of $S(k, m, n)$ containing a given element $a$ of $\Omega$, one obtains another Steiner system $S(k-1, m-1, n-1)$ on $\Omega-\{a\}$. In this way the Steiner systems for $M_{11}, M_{22}, M_{23}$ can be obtained from those of $M_{12}$ and $M_{24}$.

THEOREM 8. There exist unique Steiner triple systems $S(5,6,12)$ and $S(5,8,24)$ such that

$$
\operatorname{Aut}(S(5,6,12))=M_{12} \text { and } \operatorname{Aut}(S(5,8,24))=M_{24} \text {. }
$$

Thus as a corollary one has

COROLlary 9. There exist Steiner triple systems $S(4,5,11), S(4,7,23)$, and $S(3,6,22)$ such that

$$
\begin{aligned}
& \operatorname{Aut}(S(4,5,11))=M_{11}, \operatorname{Aut}(S(4,7,23))=M_{23}, \\
& \text { and } \operatorname{Aut}(S(2,6,22))=\operatorname{Aut}\left(M_{22}\right) .
\end{aligned}
$$

Note that $M_{22}$ is of index 2 in $\operatorname{Aut}\left(M_{22}\right)$, while $M_{23}$ and $M_{24}$ are their own automorphism groups. One also has a Steiner system $S(2,5,21)$ and $\operatorname{Aut}(S(2,5,21)) \cong \operatorname{Aut}\left(L_{3}(4)\right)$. However, the subgroup $M_{21}$ of $M_{22}$ which acts on $S(2,5,21)$ is just $L_{3}(4)\left(M_{21}\right.$ is the stabilizer of a point in $\left.M_{22}\right)$.

3. Janko's first group. The family of Ree groups ${ }^{2} G_{2}\left(3^{2 n+1}\right), n>1$, is a very interesting one. If $G^{*}$ is one of its members, then $G^{*}$ has the following properties:

(a) A Sylow 2-subgroup of $G^{*}$ is elementary abelian of order 8;

(b) If $t^{*} \in \mathscr{G}\left(G^{*}\right)$, then $C_{G^{*}}\left(t^{*}\right) \cong Z_{2} \times L_{2}\left(3^{2 n+1}\right)$;

(c) $\left|G^{*}\right|=\left(3^{n}-1\right) 3^{3 n}\left(3^{3 n}+1\right)$ and a Borel subgroup $B^{*}$ of $G^{*}$ has order $\left(3^{n}-1\right) 3^{3 n} ;$ and

(d) The permutation representation of $G^{*}$ on the cosets of $B^{*}$ is doubly transitive and some three-point stabilizer has order 2.

In attempting to characterize the Ree groups by internal properties, it is natural to ask to what extent conditions (a), (b) imply (c), (d) and also whether an arbitrary group satisfying conditions (c), (d) must be isomorphic to ${ }^{2} G_{2}\left(3^{n}\right)$. The second question will be discussed in the next chapter; here we focus on the first.

Regarded as a general question, the restriction on the order of a Sylow 2-subgroup of $G$ and on the order of the characteristic power $q$ in $L_{2}(q)$ are clearly artificial. First, H. N. Ward [215] and then Janko-Thompson [135] considered this general problem; their combined efforts yield the following result.

THEOREM 1. If $G$ is a simple group with abelian Sylow 2-subgroups and the centralizer of some involution of $G$ is isomorphic to $Z_{2} \times L_{2}(q), q \geqslant 3$, then one of the following holds: 
(i) $q=3^{n}, n$ odd, $n>1,|G|=\left(3^{n}-1\right) 3^{3 n}\left(3^{3 n}+1\right)$, and $G$ is a doubly transitive permutation group of degree $3^{3 n}+1$ in which some 3-point stabilizer has order 2; or

(ii) $q=4$ or 5 (note that $L_{2}(4) \cong L_{2}(5)$ ).

Let $S \in \operatorname{Syl}_{2}(G)$ and let $t \in G(S)$ be such that $C_{t} \cong Z_{2} \times L_{2}(q)$. Since $G$ is simple and $S$ is abelian, an elementary transfer argument implies that all involutions of $S$ and hence of $G$ are conjugate. If $q=2^{m}$, one easily derives a contradiction when $m \geqslant 3$ by analyzing $N_{G}(S)$. Hence $m=2$ and $q=4$ in this case. We can therefore suppose $q$ is odd. As $S$ is abelian, this forces $q \equiv 3,5(\bmod 8)$ and $S \cap L$ is a four group, whence $S$ is elementary of order 8. If $q=3, S \cap L \triangleleft L$ and so $S \triangleleft C_{t}$. Now a theorem of Feit [53] applies to yield a contradiction. Hence we can suppose that $q>5$. Thus $q=p^{m}, p$ odd, and the congruence on $q$ forces $m$ to be odd.

Character theory is used to conclude that

$$
|G|=q^{3}\left(q^{3}+1\right) f,
$$

where $f$ is the degree of an irreducible character of $G$. Then, assuming $p \neq 3$, a local analytic argument (which utilizes (2)) yields that some element of $C_{t}$ of order 3 centralizes an element of $C_{t}$ of order $p$, contrary to the structure of $L_{2}\left(p^{m}\right)$.

If one analyzes the case $q=5$ in the same way, one does not reach the anticipated contradiction. However, the "first few times around" numerical errors camouflaged the true situation. But eventually Janko's dogged persistence showed that everything fit together beautifully and, in particular, that such a group $G$ must have a uniquely determined character table and order 175,560 . Ultimately Janko proved the following result, and the modern theory of sporadic groups was launched [130].

THEOREM 2. If $G$ is a simple group with abelian Sylow 2-subgroups (of order 8) and the centralizer of some involution of $G$ is isomorphic to $Z_{2} \times L_{2}(5)$, then $G$ is a uniquely determined simple group of order 175,560. Moreover, $G$ is isomorphic to the subgroup of $G L(7,11)$ generated by the following two matrices $Y$ and $Z$ (with coefficients in GF(11)) of order 7 and 5, respectively:

$$
\begin{gathered}
Y=\left(\begin{array}{lllllll}
0 & 1 & 0 & 0 & 0 & 0 & 0 \\
0 & 0 & 1 & 0 & 0 & 0 & 0 \\
0 & 0 & 0 & 1 & 0 & 0 & 0 \\
0 & 0 & 0 & 0 & 1 & 0 & 0 \\
0 & 0 & 0 & 0 & 0 & 1 & 0 \\
0 & 0 & 0 & 0 & 0 & 0 & 1 \\
1 & 0 & 0 & 0 & 0 & 0 & 0
\end{array}\right), \\
Z=\left(\begin{array}{rrrrrrr}
-3 & 2 & -1 & -1 & -3 & -1 & -3 \\
-2 & 1 & 1 & 3 & 1 & 3 & 3 \\
-1 & -1 & -3 & -1 & -3 & -3 & 2 \\
-1 & -3 & -1 & -3 & -3 & 2 & -1 \\
-3 & -1 & -3 & -3 & 2 & -1 & -1 \\
1 & 3 & 3 & -2 & 1 & 1 & 3 \\
3 & 3 & -2 & 1 & 1 & 3 & 1
\end{array}\right] .
\end{gathered}
$$


Once Janko had the order and character table of the unknown group $G$, he analyzed its modular characters for each prime dividing $|G|$ and eventually showed that such a group $G$ had to possess one and only one absolutely irreducible representation of degree 7 over $G F(11)$.

One would imagine it would be easy to decide whether $\operatorname{GL}(7,11)$ contains a subgroup $G$ with the prescribed order and properties and no doubt it could be settled quickly by a computer. However, it is another matter to accomplish this by hand. Janko uses his character-theoretic information to derive further internal properties of $G$. First, $B=N_{G}(S)=H S$, where $H$ is a Frobenius group of order 21 with kernel of order 7 and complement of order 3. Moreover, if $y \in H$ has order 7 and $x \in S^{\#}, N_{G}(H)$ contains an involution $w$ such that $z=x w$ has order 5 and $C_{H}(w)$ has order 3. Furthermore, $G=$ $\langle y, z\rangle$. Representing $y$ by the matrix $Y$ above, Janko then argues, using the various relations among $y, x, w$ which he has established, that there is only one possibility for the $7 \times 7$ matrix over $G F(11)$ representing $z$ : namely, the matrix $Z$ above. At this point, uniqueness has been proved-there exists at most one group satisfying the given conditions.

However, to establish existence of such a group, it remains to determine the group $\langle Y, Z\rangle$ and to show that it satisfies the given hypotheses. This reduces without much difficulty to showing that $\langle Y, Z\rangle$ has order 175,560 . This highly arduous task was carried out by M. A. Ward. Thus $J_{1}$ uniquely exists!

The group $J_{1}$ has no doubly transitive or even rank 3 permutation representations (as follows directly from its character table), so there is no "natural" geometry associated with it. The best that has been done is to show that $J_{1}$ is a subgroup of $G_{2}(11)$ (which was proved by Coppell), but again the embedding is not a natural one. Thus no pat reason for the existence of this group has been found. The implication of this last remark is that $J_{1}$ could have been discovered only in the process of treating some general classification problem. (M. Hall has systematically determined all simple groups of order less than one million and would have hit Janko's group at 175,560 had it not already been known to exist. McKay and also several Caltech students were involved in this effort. However, this exhaustive approach is clearly limited to groups of low orders.)

4. Sporadic groups from centralizers of involutions. With the construction of $J_{1}$, it was natural to experiment with other prospective candidates for centralizers of involutions in new simple groups. Given the great effort required to construct $J_{1}$, coupled with the extremely low probability of success, it is remarkable that four further sporadic groups-Janko's groups $J_{2}$, $J_{3}, J_{4}$, and Lyons' group $L y$-have arisen as a result of just such a judicious guess. Held's group also arose from a centralizer of involution problem, but in that case the centralizer was "given" in advance.

In addition, five other groups-O'Nan's group $O N$, the Fischer baby monster $F_{2}$, the Fischer monster $F_{1}$, and its two offspring, the Harada subgroup $F_{5}$ and the Thompson subgroup $F_{3}$-each of which arose in a context distinct from centralizers of involutions, have been investigated beginning with the structure of the centralizer of one of their involutions. Thus a total of 11 of the 26 sporadic groups have been (or are in the process of being) constructed 
from such a centralizer. (Some of these groups have more than one conjugacy class of involutions.)

The following table lists each of these 11 groups with the corresponding centralizer of an involution. The new notation occurring in it will be explained directly below the table.

\begin{tabular}{cc} 
group & centralizer of an involution \\
\hline$J_{1}$ & $Z_{2} \times A_{5}$ \\
$J_{2}$ & $A_{5} / Q_{8} * D_{8}, 2$-constrained \\
$J_{3}$ & same \\
$J_{4}$ & $\left(\hat{M}_{22} /\left(D_{8}\right)^{6}\right) \cdot 2,2$-constrained \\
$H e$ & $L_{3}(2) / D_{8} * D_{8} * D_{8}, 2$-constrained \\
$L y$ & $\hat{A}_{11}$ \\
$O N$ & $\left(\hat{\hat{L}}_{3}(4)\right) \cdot 2$ \\
$F_{2}$ & $\left({ }^{2} \hat{E}_{6}(2)\right) \cdot 2$ \\
$F_{1}$ & $\hat{F}_{2}$ \\
$F_{5}$ & $(\hat{H S}) \cdot 2$ \\
$F_{3}$ & $A_{9} /\left(D_{8}\right)^{4}, 2$-constrained
\end{tabular}

Here the symbol $A / B$ denotes a group $X$ having a normal subgroup isomorphic to $B$ with corresponding factor group isomorphic to $A$; the symbol $(A) \cdot 2$ denotes a group $X$ having a normal subgroup of index 2 isomorphic to $A$ with a particular action for the elements of $X$ on this subgroup of index 2; the phrase "2-constrained" means that the group in question is 2-constrained; the symbols $\hat{M}_{22}, \hat{A}_{11}, \hat{L}_{3}(4),{ }^{2} \hat{E}_{6}(2), \hat{F}_{2}, \hat{H S}$ denote perfect central extensions of the corresponding "unhatted" groups by $Z_{3}, Z_{2}$, $Z_{4}, Z_{2}, Z_{2}, Z_{2}$, respectively; and the symbol $H S$ denotes the Higman-Sims sporadic simple group, which will be discussed in $\$ 6$.

We make a few comments. Except in the $L_{3}(4)$ case, there is only one possibility for the given extension. However, $L_{3}(4)$ has two nonisomorphic perfect central extensions by $Z_{4}$, precisely one of which occurs in O'Nan's group. It is interesting to note that in He the centralizer of a non 2-central involution is of the form $\left(\hat{L}_{3}(\hat{4})\right) \cdot 2$, where the symbol $\hat{L}_{3}(\hat{4})$ denotes the (unique) perfect central extension of $L_{3}(4)$ by $Z_{2} \times Z_{2}$.

Apart from the groups $F_{1}, F_{2}$, and $F_{5}$, the involution specified in the table is 2-central. The centralizer of a 2-central involution in these three groups is as follows:

$$
\begin{array}{ccc}
F_{1} & \cdot 1 /\left(D_{8}\right)^{12}, & \text { 2-constrained } \\
F_{2} & \cdot 2 /\left(D_{8}\right)^{11}, & \text { 2-constrained } \\
F_{5} & \left(A_{5} / Z_{2}\right) /\left(D_{8}\right)^{4}, & \text { 2-constrained }
\end{array}
$$

Here the symbols .1, .2 denote Conway's first and second simple groups, which will be discussed in $\$ 8$. 
I would like to explain now the precise context in which each of these groups has arisen, with the exception of the already discussed $J_{1}$ and the baby monster $F_{2}$, which is best left to a discussion of Fischer's general theory of groups generated by a conjugacy class of "transpositions".

A. JANKO's GROUPS $J_{2}, J_{3}$. I do not know what impulse prompted Janko to choose $A_{5} / Q_{8} * D_{8}$ as a propitious centralizer. It does have the virtue of being a small group, distinct from the centralizer of an involution in any then known simple group, yet closely resembling such a centralizer (e.g., $L_{4}(2) \cong$ $A_{8}$ has $\Sigma_{3} / Q_{8} * Q_{8}$ as centralizer of an involution). Janko proved

THEOREM 1 . If $G$ is a simple group in which the centralizer of an involution is isomorphic to $A_{5} / Q_{8} * D_{8}$, then one of the following holds:

(i) $G$ has two classes of involutions and $|G|=2^{7} \cdot 3^{3} \cdot 5^{2} \cdot 7$; or

(ii) $G$ has one class of involutions and $|G|=2^{7} \cdot 3^{5} \cdot 5 \cdot 17 \cdot 19$.

Furthermore, as is the case for most of the groups to be discussed here, Janko determined the complete local structure of such a group $G$ as well as its character table in each case. However, this result, in contrast with his work on $J_{1}$, does not tell you whether there exists a simple group of either order or even whether there is at most one such group. All we can say is that Janko's work provides "strong evidence" for the existence of simple groups of the specified orders; for is it likely, if none existed, that such a detailed analysis could have been carried out without reaching some internal contradiction? Until now, at least, "strong evidence" has always led to the existence of an actual group. The difficulties with Janko's group $J_{4}$ and the Fischer monster $F_{1}$ is not that one expects no group to exist, but rather that because of their large orders, it is not easy to carry out the necessary computer calculations required for their construction.

We postpone until the next two sections a discussion of the actual construction of $J_{2}$ and $J_{3}$ as well as the other eight groups on the list.

B. HELD's GROUP $\mathrm{He}$. Held began with the following interesting fact: The groups $L_{5}(2)$ and $M_{24}$ have 2-central involutions with isomorphic centralizers. It was natural to attempt to characterize these groups by this property; and this was Held's intent when he began the problem. However, in his analysis of the conjugacy classes of involutions he was led to three distinct, but selfconsistent fusion patterns. He ultimately proved [112]:

THEOREM 2. If $G$ is a simple group in which the centralizer of an involution is isomorphic to $L_{3}(2) / D_{8} * D_{8} * D_{8}$, then one of the following holds:

(i) $G \cong L_{5}(2)$ or $M_{24}$; or

(ii) $G$ has exactly two conjugacy classes of involutions and $|G|=2^{10} \cdot 3^{3} \cdot 5^{2}$. $7^{3} \cdot 17$.

Again Held determines the full internal structure and a good deal of the character table. Likewise the theorem says nothing about the existence and uniqueness of a group satisfying (ii). In the next chapter we shall describe the general methods by which one can identify a known simple group from its internal structure. Obviously some procedure is necessary for Held to be able to conclude that $G \cong L_{5}(2)$ or $M_{24}$.

C. LyONs' GROUP $L y$. McLaughlin's group Mc (to be described in the next 
section) arises from a primitive rank 3 permutation group problem. However, once $M c$ was constructed, its internal structure could obviously be examined. It has only one conjugacy class of involutions and the centralizer of an involution is isomorphic to $\hat{A}_{8}\left(\hat{A}_{n}\right.$ denotes the perfect central extension of $A_{n}$ by $Z_{2}$ ). This was an intriguing answer since it immediately suggests studying the class of groups $G$ having $\hat{A}_{n}$ as centralizer of an involution $t$ for any $n \geqslant 5$. From the structure of a Sylow 2-subgroup $T_{n}$ of $\hat{A}_{n}$, it is immediate that such an involution $t$ must be 2-central in $G$. Also by order considerations, $T_{n} \cong T_{n+1}$ for $n$ even.

Thompson then made the interesting observation that the group $T_{10}(\cong$ $T_{11}$ ), of order $2^{8}$, appears on MacWilliams list of 2-groups of normal 2-rank 2 which are possible candidates for Sylow 2-subgroups of some simple group, as an exceptional case [142]. Here then was the place to begin! And Thompson suggested the problem to his student Lyons, who proved [141]

THEOREM 3. If $G$ is a simple group in which the centralizer of some involution is isomorphic to $\hat{A}_{n}, n=10$ or 11 , then $n=11, G$ has only one conjugacy class of involutions, and

$$
|G|=2^{8} \cdot 3^{7} \cdot 5^{6} \cdot 7 \cdot 11 \cdot 31 \cdot 37 \cdot 67 .
$$

The following result shows that there is no more gold to be found in this direction!

THEOREM 4. If $G$ is a simple group in which the centralizer of some involution is isomorphic to $\hat{A}_{n}, n \geqslant 5$, then $n=8$ or 11 .

The cases $n=5,6,7$ follow from a theorem of Brauer and Suzuki on groups with quaternion Sylow 2-subgroups [32] (cf. Theorem IV.5.5). The case $n=9$, due to Janko [132] requires a difficult analysis not unlike that carried out by Lyons. However, when $n \geqslant 12$, a more uniform argument can be given. This was done by Thompson and also by R. Solomon [178].

D. O'NAN's GROUP ON. Some years ago, Alperin and I were investigating simple groups of 2-rank 3. This work led Alperin to analyze the possible 2-constrained groups of the form $L_{3}(2) /\left(Z_{2^{n}} \times Z_{2^{n}} \times Z_{2^{n}}\right)$ for some $n$. He proved [2]:

THEOREM 5. For each value of $n \geqslant 1$, there are (up to isomorphism) exactly two 2-constrained groups of the form $L_{3}(2) /\left(Z_{2^{n}} \times Z_{2^{n}} \times Z_{2^{n}}\right)$, in one of which the extension splits and in the other the extension does not split. Moreover, each nonsplit group has 2-rank 3.

Denote these groups by $A l p_{n}^{1}$ and $A l p_{n}^{2}$, respectively. The group $A l p_{1}^{1}$ occurs as a 2-local (mod core in the case of $A_{11}$ ) in $A_{8}, A_{9}, A_{10}, A_{11}, M_{22}, M_{23}$, the group $A l p_{1}^{2}$ in $G_{2}(q)$ and ${ }^{3} D_{4}(q), q$ odd, and the group $A l p_{2}^{1}$ in $H S$. Here then is another natural question: For what values of $n \geqslant 2$ do the groups $A l p_{n}^{1}$ or $A l p_{n}^{2}$ occur as 2-local subgroups of a simple group $G$ ? Note that for $n \geqslant 2$, it is easily shown that $O_{2}\left(A l p_{n}^{i}\right)$ is the unique subgroup of $A l p_{n}^{i}$ of type $Z_{2^{n}} \times Z_{2^{n}}$ $\times Z_{2^{n}}$ and so $A l p_{n}^{i}$ must then necessarily contain a Sylow 2-subgroup of $G$. Thus for a given $n \geqslant 2$ and $i=1$ or $2, G$ has a uniquely determined Sylow 2-subgroup. 
I was hoping to interest a doctoral student in this problem; but O'Nan was forced to consider it in connection with his work on doubly transitive permutation groups of odd degree in which the one-point stabilizer has a nontrivial normal subgroup of odd order [153]. That analysis ultimately reduced to determining all groups $G$ of 2-rank $r, r$ a positive integer, such that for some elementary abelian 2-subgroup $A$ of $G$ of $\operatorname{rank} r, N=N_{G}(A)$ transitively permutes the flags of $A$. Here, by definition, a flag of $A$ is a nested sequence of subspaces

$$
1<A_{1}<A_{2}<\cdots<A_{r}=A .
$$

(Thus, $A_{i}$ is of codimension 1 in $A_{i+1}, 1 \leqslant i \leqslant r-1$.) To say that $N$ acts transitively on the flags of $A$ means that for any two flags, some element of $N$ conjugates the subspaces of the first into the corresponding subspaces of the second.

In dealing with the case $r=3$, one of the possibilities is that $N / O(N) \cong$ $A l p_{n}^{i}$. If $n=1$, then $A \in \operatorname{Syl}_{2}\left(C_{G}(A)\right)$ and a theorem of Harada (to be discussed at the end of Chapter V) implies that $G$ is isomorphic to one of the groups listed above (assuming $G$ is simple). Thus O'Nan could restrict himself to the case $n \geqslant 2$, in which case $G$ has a specified Sylow 2-group for each $n$ and $i$.

O'Nan proved [155], using a characterization of $H S$ by its Sylow 2-group previously obtained by Harris and me [90]:

THEOREM 6. If $G$ is a simple group with Sylow 2-subgroup isomorphic to that of $A l p_{n}^{i}, n \geqslant 2, i=1$ or 2 , then $n=2$ and one of the following holds:

(i) $i=1$ and $G \cong H S$; or

(ii) $i=2, G$ has only one conjugacy class of involutions, and

$$
|G|=2^{9} \cdot 3^{4} \cdot 5 \cdot 7^{3} \cdot 11 \cdot 19 \cdot 31 .
$$

E. JANKo'S FOURTH GROUP. The reader will certainly have observed that several of the eleven groups listed in the table of the previous section are of $G F(2)$-type. Moreover, as we have already pointed out, such groups play a basic role in the general theory of simple groups. For some time now, it has been felt that if any new sporadic simple groups are to be found, this is the place to look for them. Timmesfeld's fundamental work which we shall describe in Chapter VII (combined with a result of S. Smith [177] which completes a case only partially resolved by Timmesfeld) shows that those of sufficiently large width are necessarily classical groups over $G F(2)$. Thus the search was forced to focus on the narrow width case. It was out of such investigations that Janko was led to his fourth group [134]. However, very recently S. Smith [177] has completed the analysis in all remaining low width cases and has shown that there are no further sporadic groups to be found in this direction!

Janko's main result is as follows:

THEOREM 7. If $G$ is a simple group in which the centralizer of some involution is isomorphic to $\left(\hat{M}_{22} /\left(D_{8}\right)^{6}\right) \cdot 2$, then $G$ has exactly two conjugacy classes of involutions and

$$
|G|=2^{21} \cdot 3^{3} \cdot 5 \cdot 7 \cdot 11^{3} \cdot 23 \cdot 29 \cdot 31 \cdot 37 \cdot 43 .
$$


Janko determined that the centralizer of an involution of the second class has to be a nontrivial split extension of an elementary group $E$ of order $2^{11}$ by $\operatorname{Aut}\left(M_{22}\right)$. Furthermore, he showed that a maximal 2-local subgroup containing this centralizer is necessarily a split extension of $E$ by $M_{24}$. In particular, any such group $G$ must be of characteristic 2 type. At this time it is unknown whether there actually exists a group satisfying these conditions.

F. THE FISCHER MONSTER AND ITS SUBGROUPS. Out of his work on the baby monster $F_{2}$, Fischer was led to believe that there might be a simple group $G$ having $\hat{F}_{2}$ as centralizer of an involution. In such a group $G$, a likely candidate for the centralizer of a certain element of order 3 would have the form $\widehat{S u z} / X$, where $\widehat{S u z}$ denotes the cover by $Z_{2}$ of the Suzuki sporadic group (to be described in 86 ) and $X$ is extra-special of order $3^{13}$, the extension being 2-constrained. (The group $\widehat{S u z}$ is known to have a faithful 12-dimensional modular representation over $G F(3)$.) Examining the centralizer in $G$ of the involution corresponding to a generator of $Z(S u z)$, one can then argue that it must have the form $.1 /\left(D_{8}\right)^{12}$. Remarkably these calculations were carried out independently at the same time by Griess in Michigan and Conway and Thompson in Cambridge, England. By a prodigious calculation, using the Thompson order formula (Theorem 13 below), Griess determined the exact order of such a group $G$ [100]. (Conway and Thompson also obtained this value, but only as a possible lower bound for the order of $G$.) Griess' result is as follows:

THEOREM 9. Let $G$ be a simple group containing involutions $x, y$ such that $C_{x} \cong \hat{F}_{2}$ and $C_{y} \cong 1 /\left(D_{8}\right)^{12}$. Then $G$ has exactly two conjugacy classes of involutions and

$$
|G|=2^{46} \cdot 3^{20} \cdot 5^{9} \cdot 7^{6} \cdot 11^{2} \cdot 13^{3} \cdot 17 \cdot 19 \cdot 23 \cdot 29 \cdot 31 \cdot 41 \cdot 47 \cdot 59 \cdot 71 .
$$

S. Smith [177] has very recently shown that the structure of $C_{x}$ and hence the order of $G$ follows from the structure of $C_{y}$ alone.

Thompson was further able to show that if such a group $G$ existed, then the centralizers in $G$ of certain elements of order 3 and 5 would have direct factors (of index 3 and 5, respectively), which were themselves new simple groups. Moreover, he determined likely candidates for centralizers of involutions in these groups: namely, $A_{9} /\left(D_{8}\right)^{4}$ and $(\widehat{H S}) \cdot 2$, respectively. The suggestive terminology $F_{3}$ and $F_{5}$ for these two groups has now become standard, the ambient group $G$ being denoted by $F_{1}$ and dubbed the "monster".

With P. Smith's aid on the computer at the University of Cambridge, Thompson has proved the existence and uniqueness of $F_{3}$ [205] and Harada (with the assistance of $S$. Norton) the existence and uniqueness of $F_{5}$ [110]. For the present, we content ourselves with the following statements.

THEOREM 10. If $G$ is a simple group in which the centralizer of some involution is isomorphic to $A_{9} /\left(D_{8}\right)^{4}$, then $G$ has only one conjugacy class of involutions and

$$
|G|=2^{15} \cdot 3^{10} \cdot 5^{3} \cdot 7^{2} \cdot 13 \cdot 19 \cdot 31
$$


THEOREM 11. If $G$ is a simple group in which the centralizer of some involution is isomorphic to $(\widehat{H S}) \cdot 2$, then $G$ has two conjugacy classes of involutions and

$$
|G|=2^{14} \cdot 3^{6} \cdot 5^{6} \cdot 7 \cdot 11 \cdot 19
$$

Griess also established some additional local properties of $F_{1}$ and certain information about its character table. In particular, the minimum degree of a complex representation of $F_{1}$ is 196,883 . This fact, combined with its order (approximately $10^{54}$ ) certainly makes the term "monster" an appropriate appellation!

Since then Norton has determined the conjugacy classes of $F_{1}$ (up to a few indeterminacies) and also many properties of its purported character $\chi$ of degree 196,883 [150]. In particular, he has shown that $\chi$ is rational-valued. In fact, if $F_{1}$ exists, $\chi$ must occur as a constituent of the character of $G$ "induced" from the trivial character $1_{\hat{F}_{2}}$ of its subgroup $\hat{F}_{2}$. Likewise he has shown that $\chi$ occurs precisely once as a constituent of its "symmetric square", which implies that the corresponding module for $F_{1}$ has the structure of a (nonassociative) algebra. (So far at least it has not been possible to find any obvious identities satisfied by this algebra.) I mention these facts as indications of possible directions for attacking the extremely difficult question of the existence of the monster. (More recently, Livingston and Fischer have completed determination of the full character table of $F_{1}$, assuming the existence of an irreducible representation of degree 196, 883.)

Let me also say something concerning the method of determining the order of one of these groups $G$ from the structure of the centralizer of an involution. The first step is to obtain, by 2-local analysis, the precise possibilities for the fusion pattern of involutions of $G$. If $G$ has more than one class of involutions, the next step is to determine the exact structure of the centralizers of involutions in the remaining classes. In this case, one is now in a position to apply the so-called "Thompson order formula" directly to compute the order of $G$. This formula, which is established by very elementary counting arguments, can be viewed as a refinement of classical results of Brauer and Fowler [30] on properties of involutions in groups of even order.

For simplicity, we state Thompson's formula only for groups having exactly two conjugacy classes of involutions. We need a definition.

Definition 12 . Let $G$ be a group with exactly two conjugacy classes of involutions, represented by the involutions $x$ and $y$. For any involution $z$ of $G$, define $a(z)$ to be the number of ordered pairs $(u, v)$ of involutions $u$, $v \in G$ such that $u$ is conjugate to $x$ and $v$ to $y$ in $G$ and such that $(u v)^{i}=z$ for some integer $i$.

Note that as $\langle u, v\rangle$ is a dihedral group, it follows that $z$ centralizes both $u$ and $v$ for any such pair $(u, v)$. Hence $a(z)$ is determined entirely within $C_{z}$ and can be computed once the exact fusion pattern of involutions in $G$ is known.

THEOREM 13. If $G$ is a group with exactly two conjugacy classes of involutions, represented by the involutions $x$ and $y$, then 


$$
|G|=a(y)\left|C_{x}\right|+a(x)\left|C_{y}\right| .
$$

When $G$ has only one conjugacy class of involutions, the procedure is considerably more complicated. One first determines the $p$-local structure of $G$ (including the conjugacy classes of elements of order $p$ ) for the set of "visible" odd primes-namely, those dividing the order of the given centralizer of an involution. This determination makes use of the 2-local structure of $G$ and involves prior classification theorems. (Such theorems are usually also involved in determining the possible involution fusion patterns of $G$ and its 2-local structure.)

With this information, one can now obtain a congruence for the order of $G$ with the aid of Sylow's theorem and a result of Frobenius concerning the number of solutions of the equation $x^{n}=1$ in a group. However, in practice, several local structures may be possible at this stage. If we take O'Nan's group as an illustration, two local structures may occur, one in which $G$ has Sylow 7-subgroups of order 7 (Case I) and the other of order $7^{3}$ (Case II) [155]. Correspondingly O'Nan obtains:

$$
\begin{aligned}
& |G|=2^{9} \cdot 3^{4} \cdot 5 \cdot 7 \cdot m, \quad \text { where } m \equiv 939551\left(\bmod 2^{9} \cdot 3^{4} \cdot 5 \cdot 7\right) \quad(\text { Case I); } \\
& |G|=2^{9} \cdot 3^{4} \cdot 5 \cdot 7^{3} \cdot m, \quad \text { where } m \equiv 6479\left(\bmod 2^{9} \cdot 3^{4} \cdot 5 \cdot 7^{3}\right) \quad \text { (Case II). }
\end{aligned}
$$

To determine $m$, one must get at the "invisible" primes-those dividing $m$. One is primarily interested in those which are represented by "strongly real" elements-i.e., elements which are inverted by some involution of $G$. These strongly real elements break up into $n$ disjoint sets, corresponding to certain abelian subgroups of $G$, with each of which one can associate a certain number $w_{i}$ of exceptional characters of $G, w_{i}$ being determined from the normalizer of the corresponding abelian subgroup. Using elementary character theory and counting arguments (including a result of Brauer and Fowler), O'Nan is now able to prove in succession

$$
m \leqslant 2^{9} \cdot 5 \cdot 7\left(23+\sum_{i=1}^{n} w_{i}\right) .
$$

(23 is the total number of strongly real classes of elements of order 2, 3, 5 and 7.)

$$
\begin{aligned}
n & \leqslant 5 . \\
w_{i} & \leqslant 45 . \\
m & \leqslant 4,500,000 .
\end{aligned}
$$

Now using (1) in Case I, there are only three possible values of $m$ satisfying (5): namely, $m=939551,2391071$, or $3842591=71 \cdot 54121$, the first two being primes. Sylow's theorem yields a contradiction in the first two cases. In the third case, one has $n \leqslant 2$, giving the sharper estimate $m \leqslant 2400000$, contradiction.

Hence Case II must hold. This time, using the bound for $m$ and (1), the only solution is $m=6479=11 \cdot 19 \cdot 31$ and the order of $G$ is uniquely determined. 
For simplicity, we shall say that any group $G$ having one of these centralizers of an involution plus any set of further properties derivable from this assumption (such as order, local structure, character table, etc.) is of type-. Thus we have groups of type $J_{i}, 1 \leqslant i \leqslant 4$, type $H e$, type $L y$, type $O N$, and type $F_{i}, 1,2,3$, or 5 .

To distinguish type $J_{2}$ from type $J_{3}$, we must, of course, include the condition on the number of conjugacy classes of involutions.

In this terminology, Theorem 3.2 asserts that there is a unique simple group of type $J_{1}$.

5. Computer construction of sporadic groups. We must now address the question of the existence (and uniqueness) of simple groups $G$ of each of the above ten types (excluding $J_{1}$ ). Apart from the group $J_{2}$, the answer in each case seems to require the assistance of a high speed computer. On the other hand, $J_{2}$ has a natural geometry arising from its representation as a rank 3 permutation group, on the basis of which one can construct it directly. This will be described in the next section. The groups $F_{2}, F_{3}$, and $F_{5}$ also have certain geometries associated with them, but because of their size it does not seem possible to make the necessary calculations for their construction by hand. The remaining groups appear to have no associated natural geometries.

From the local analysis, one can obtain rather complete information about the local structure of a group $G$ of one of these types. Then, using the theory of induced characters-in particular, Brauer's theorem which asserts that every irreducible character of $G$ is a $\mathbf{Z}$-linear combination of characters induced from so-called "elementary" subgroups [34, Theorem 4.7.1]-one is able to calculate a substantial portion and, in some cases all, of the character table of $G$. Thus the real question we are asking is the following:

How does one construct a (simple) group $G$ from

(a) Its local structure; and

(b) Its character table?

The first point to be made is that we may suspect that our unknown group $G$ must also contain certain nonlocal subgroups. The groups $J_{2}, J_{3}, H e, L y$, $O N$, and $F_{5}$ each possess such a nonlocal subgroup. Moreover, what is even more important, each of these groups has been constructed as a permutation group on the cosets of that subgroup. Why should we imagine that our group $G$ contains a particular such subgroup-e.g., that a group of type $L y$ must have a subgroup isomorphic to $G_{2}(5)$ ? We know that $G_{2}(5)$ is generated by two of its local subgroups $A^{*}, B^{*}$. Suppose our local information about a group $G$ of type $L y$ tells us that $G$ must contain subgroups $A, B$ isomorphic to $A^{*}, B^{*}$, respectively. Isn't it then reasonable to conjecture that for a suitable choice of $A, B$, it will be the case that $\langle A, B\rangle \cong G_{2}(5)$ ?

Under such circumstances, there is a very nice procedure which often enables one to answer the question in the affirmative, known as the "Brauer trick". In fact, apart from generator and relation calculations, it is essentially the only known method for proving the existence of nonlocal subgroups. To explain the procedure, suppose first that the desired subgroup $H$ of $G$ exists. Then the complex representation corresponding to the permutation representation on the cosets of $H$ decomposes into irreducible constituents. Let $\chi$ be the character of a nontrivial such irreducible constituent. Then $\chi$ is a 
constituent of $\left(1_{H}\right)^{G}$, the character of $G$ induced from the trivial character $1_{H}$ of $H$. Hence by the Frobenius reciprocity theorem, [84, Theorem 4.4.5] $1_{H}$ must be a constituent of the restriction $\chi_{H}$ of $\chi$ to $H$. It is this observation which underlies Brauer's procedure.

We begin with our subgroups $A$ and $B$ of $G$ (for this purpose we can assume $G$ exists). From the character table of $G$, we can determine a likely irreducible character for $\chi$. We let $\Re$ be the representation associated with $\chi$, acting on the (complex) vector space $V$. For each subgroup $Y$ of $G$, we denote by $V_{Y}$, the subspace of $V$ on which $\Re(Y)$ acts trivially and we set $d_{Y}=$ $\operatorname{dim} V_{Y}$. Thus $d_{Y}$ is the multiplicity of $1_{Y}$ in $\chi_{Y}$. The integer $d_{Y}$ is given by the following inner product:

$$
d_{Y}=\left(\chi_{Y}, 1_{Y}\right) .
$$

If we have sufficient information about the character table of $G$ and of the subgroup $Y$, we can compute $d_{\boldsymbol{Y}}$.

To apply the Brauer trick, we must be able to compute $d_{A}, d_{B}$, and $d_{C}$, where $C=A \cap B$. Since $C \leqslant A$ and $C \leqslant B$, we have $V_{A} \leqslant V_{C}$ and $V_{B} \leqslant$ $V_{C}$, so by counting dimensions of subspaces of $V_{C}$, we have

$$
\operatorname{dim}\left(V_{A} \cap V_{B}\right)+d_{C} \geqslant d_{A}+d_{B} .
$$

If our calculations should yield that $d_{A}+d_{B}>d_{C}$, we can conclude from (2) that $V_{A} \cap V_{B} \neq 0$. This is the goal of the Brauer method, for it immediately implies that $H=\langle A, B\rangle$ is a proper subgroup of $G$. Indeed, as $\Re(H)$ acts trivially on $V_{A} \cap V_{B} \neq 0$ and $\Re$ is a nontrivial irreducible representation of $G, \Re(H) \neq \Re(G)$, so $H<G$. (Of course, $\langle A, B\rangle$ may be a proper subgroup of $G$ even if $d_{A}+d_{B} \leqslant d_{C}$; but in that case we cannot verify this fact by the Brauer trick.)

The final step in the process requires the identification of the subgroup $H$. This is usually accomplished by invoking some prior classification theorem. Thus in the case of a group $G$ of type $L y$, Lyons was able to prove [141], using the subgroups $A$ and $B$ and the information he had already obtained about $G$, that $H$ had only one class of involutions and the centralizer of an involution in $H$ was isomorphic to that in $G_{2}(5)$ (it has a subgroup of index 2 isomorphic to $S L(2,5) * S L(2,5))$. Now he could invoke a theorem of Fong and W. Wong [66] to conclude that $H \cong G_{2}(5)$.

Using the Brauer procedure, the following results have been obtained.

Proposition 1. If $G$ is a group of type $J_{2}, H e, L y, O N$, or $M(24)^{\prime}$, then correspondingly $G$ contains a subgroup isomorphic to $U_{3}(3), S p(4,4)^{*}, G_{2}(5)$, $\mathrm{L}_{3}(7)^{*}$, or $\mathrm{He}$.

Here $S p(4,4)^{*}$ and $L_{3}(7)^{*}$ denote, respectively, the split extension of $S p(4,4)$ by a field automorphism of order 2 and of $L_{3}(7)$ by the transposeinverse automorphism of order 2.

On the other hand, the generator-relation method has yielded the following results.

Proposition 2. If $G$ is a group of type $J_{3}$ or $F_{5}$, then correspondingly $G$ contains a subgroup isomorphic to $S L(2,16)^{*}$ or $A_{12}$.

Here $S L(2,16)^{*}$ denotes the split extension of $S L(2,16)$ by a field 
automorphism of order 2. It was Thompson who proved the existence of this subgroup of $J_{3}$ (see [117]). G. Higman and McKay [117], [145], who constructed $\mathrm{J}_{3}$ (and also $\mathrm{He}$ ), using character-theoretic analysis and computer calculations, were only able to give strong evidence that a group of type $J_{3}$ must have such a subgroup, but could not settle the existence question. On the other hand, Harada was able to prove the existence of $A_{12}$ in a group of type $F_{5}$ fairly directly from the internal properties of such a group [110].

Since the Fischer groups $M(22), M(23), M(24)$, and $F_{2}$ are constructed as permutation groups on the cosets of the centralizer of an involution in the distinguished conjugacy class, the existence of nonlocal subgroups in these cases does not enter into the analysis.

The Higman-McKay construction of $J_{3}$ was carried out by a more or less direct process of coset enumeration, leading to a presentation by generators and relations. This method is very effective for permutation groups of not too high a degree (e.g., $J_{3}$ is of degree 6156 on the cosets of an $S L(2,16)^{*}$ subgroup). However, more elaborate computer techniques are required to construct permutation groups of large degree (ON has degree 122760, Ly has degree approximately 9 million, and the baby monster has degree approximately 13 billion). Out of his construction of $L y, O N$, and $F_{2}$ (the third group jointly with Leon), Sims has gradually developed a general technique for constructing permutation groups of large degree by computer methods [140], [172], [113]. To appreciate the technical difficulties involved, consider the fact that a modern computer can store just one permutation of the set 1 , $2, \ldots, N$, when $N$ is in the range of 150,000 to 200,000 . Using secondary storage devices one can hold much more information; however, access to such devices is comparatively slow. Hence, although it is easy enough to multiply two such permutations, the programming for carrying this out can be quite tricky. It is clear that to perform such multiplications many times, as will certainly be required to construct the required group $G$, a number of very effective algorithms will have to be developed, which require not only a profound understanding of both the computer and finite group theory, but also a high order of imaginative insight.

In order to begin, Sims must have a precise description of the representation of the proposed one-point stabilizer $H$ on its coset space. For example, O'Nan provided this information for his group by establishing the following result, based on an analysis of the conjugacy classes of elements and the character table of a group of O'Nan type.

Proposition 3. If $G$ is a group of type $O N$ and $H$ is the subgroup of $G$ isomorphic to $\mathrm{L}_{3}(7)^{*}$ constructed in Proposition 1, then we have

(i) $|G: H|=122760$ and the representation of $G$ on the cosets of $H$ has rank 5;

(ii) The nontrivial orbits $\Delta_{i}, 1 \leqslant i \leqslant 4$, of $H$ on its coset space have length 6384, 5586, 58653, and 52136, respectively; and

(iii) If $a_{i}$ is a point of $\Delta_{i}$ and $H_{i}$ is the subgroup of $H$ fixing $a_{i}, 1 \leqslant i \leqslant 4$, then we have

(1) $H_{1}$ is a semidirect product of $Z_{7} \times Z_{7}$ by $Z_{3} \times Z_{2} \times Z_{2}$;

(2) $H_{2} \cong Z_{2} \times P G L(2,7) \cong C_{H}(t)$, where $t \in G(H)-g\left(H^{\prime}\right)$; 
(3) $\mathrm{H}_{3}$ is a Sylow 2-subgroup of $\mathrm{H}$; and

(4) $H_{4} \cong Z_{3} \times \Sigma_{4}$.

This information describes the required action of $H$ on its coset space. (We note that a group $G$ of type $O N$ possesses a second conjugacy class of subgroups $H^{*} \cong L_{3}(7)^{*}$ and if we consider the representation of $G$ on the cosets of $H^{*}$, we obtain a similar, but not identical description for the action of $H^{*}$.)

Thus, Sims begins with a group $H$, a collection of subgroups $H=H_{0}$, $H_{2}, \ldots, H_{n}$, the coset spaces $\Delta_{i}=H / H_{i}$ of $H_{i}$ in $H, 0 \leqslant i \leqslant n$, and he considers the set

$$
\Omega=\bigcup_{i=0}^{n} \Delta_{i}
$$

The problem then is to construct a transitive permutation group $G$ on $\Omega$ having $H$ as its one-point stabilizer with the specified action of $H$ on $\Omega$. (The subgroups $H_{i}$ will be precisely the set of two-point stabilizers of $G$ on the corresponding orbits of $H$.)

There are four major components to the computer analysis.

(A) Algorithms for determining the order of a group generated by a given set of permutations.

(B) Techniques for defining permutations on large sets.

(C) Techniques for verifying relations satisfied by permutations defined as in (B).

(D) Ad hoc methods for finding the right permutations to generate the group in question.

Let $X$ be a set of permutations of a set $\Omega$. Under (A), one is interested in algorithms for determining the order of the group $G$ generated by $X$. Let $a \in \Omega$ and let $\Delta$ be the orbit of $a$ under $G$. The elements of $\Delta$ can be determined easily from a knowledge of $X$. Let $X_{a}$ be the subset of $X$ consisting of those permutations fixing $a$ and set $H_{a}=\left\langle X_{a}\right\rangle$. Obviously $H_{a} \leqslant G_{a}$, the subgroup of $G$ fixing $a$. The approach is to try to show that either $H_{a}=G_{a}$ or else to produce an element $y \in G_{a}-H_{a}$. In the latter case, one replaces $X$ by $X \cup\{y\}$ and repeats the process.

There are two procedures for carrying this out.

The Schreier method. For each $b \in \Delta$, choose an element $u(b)$ in $G$ taking $a$ to $b$. For each $b$ in $\Delta$ and $x \in X$, form the element

$$
y(b, x)=u(b) x\left(u\left(b^{x}\right)^{-1}\right),
$$

where $b^{x}$ is the image of $b$ under $x$.

Proposition 4. $H_{a}=G_{a}$ if and only if every $y(b, x) \in H_{a}$.

The Schreier-Todd-Coxeter method. Determine some set of relations satisfied by the elements in $X$ and let $G^{*}$ be the abstract group generated by $X$ defined by these relations. Let $H^{*}$ be the subgroup of $G^{*}$ generated by the subset $X_{a}$.

Proposition 5. If $\left|G^{*}: H^{*}\right|=|\Delta|$, then $G_{a}=H_{a}$.

Computation of $\left|G^{*}: H^{*}\right|$ is carried out by the standard methods of what 
is called "coset enumeration". If this coset enumeration does not show that $\left|G^{*}: H^{*}\right|=|\Delta|$, one can find elements $y_{1}^{*}, y_{2}^{*} \in G^{*}$ such that $H^{*} y_{1}^{*}$ and $H^{*} y_{2}^{*}$ appear to be distinct cosets of $G^{*}$, but if $y_{1}, y_{2}$ denote the corresponding elements of $G$, then the cosets $G_{a} y_{1}$ and $G_{a} y_{2}$ are equal. Form $y=$ $y_{1} y_{2}^{-1}$, so that $y \in G_{a}$. If $y$ is not in $H_{a}$, express $y$ as a product of the elements of $X$ and add this word as a new relation.

To carry this out, one normally assumes that one has first obtained a presentation for $H_{a}$ in terms of the generating set $X_{a}$.

Now for (B). There are two methods for defining permutations on a large set $\Omega$ in such a way that one can work with them effectively on a computer. The first of these is to begin with a group $H$ and subgroups $H_{i}$, as above, and then to form the coset space $\Omega=\cup \Delta_{i}$, as in (3). If $K$ is any subgroup of $H$, one can determine the orbits of $K$ on $\Omega$ when $|H: K| \leqslant 200,000$. The orbits of $K$ on a given $\Delta_{i}$ are, in fact, in one-one correspondence with the orbits of $H_{i}$ on the coset space of $K$ in $H$. This is the first method.

Suppose next that we have defined a permutation group $K$ on a set $\Omega$ and we know the orbits $\Gamma_{1}, \Gamma_{2}, \ldots, \Gamma_{m}$ of $K$ on $\Omega$. Assume now that we are given points $a_{i}, b_{i} \in \Gamma_{i}, 1 \leqslant i \leqslant m$, a permutation $\pi$ of the set $\{1,2, \ldots, m\}$, and an automorphism $\sigma$ of $K$ (as an abstract group), satisfying the following condition for each $i, 1 \leqslant i \leqslant m$ :

$$
\left(K_{a_{i}}\right)^{\sigma}=K_{b_{\pi}(i)}
$$

Here $K_{c}$ denotes, as usual, the subgroup of $K$ fixing the point $c \in \Omega$. Thus $\sigma$ transforms the one-point stabilizers of $K$ in a well-defined fashion.

Proposition 6. Under the assumption of (4), there exists a unique permutation $z$ of $\Omega$ such that

(i) $z$ normalizes $K$ and induces by conjugation the given automorphism $\sigma$ of $K$; and

(ii) $\left(a_{i}\right)^{2}=b_{\pi(i)}, 1 \leqslant i \leqslant m$.

For example, Leon and Sims [140] constructed the baby monster $F_{2}$ by beginning with the subgroup $H \cong \operatorname{Aut}(M(22))$, which they believed to be a subgroup of $\left({ }^{2} \hat{E}_{6}(2)\right) \cdot 2$, the centralizer of an involution in a group of type $F_{2}$. (Their construction proved that $M(22)$ is indeed a subgroup of ${ }^{2} E_{6}(2)$.) They then determined approximately 40 subgroups $H_{i}$ of $H$ in roughly the same way as described above for a group of type $O N$. They then constructed the coset space $\Omega$ from $H$ and its subgroups $H_{i}$ as in (3) above, the order of $\Omega$ being approximately $13 \cdot 10^{9}$. At this point, they took two subgroups for $K$; namely, $K_{1}=H$ and $K_{2}$ a subgroup of $H$ of index approximately 145,000. Then by the method just described, they constructed two permutations $z_{1}, z_{2}$ on $\Omega$ relative to $K_{1}, K_{2}$, respectively with $z_{1}$ centralizing $K_{1}$ and $z_{2}$ centralizing $K_{2}$. Ultimately they showed that the group $\left\langle H, z_{1}, z_{2}\right\rangle$ had the required order.

Now consider (C). In order to use the Schreier-Todd-Coxeter method to determine the order of the group generated by a set of permutations defined as in (B), one needs to be able to verify relations satisfied by them. Take the baby monster as an example. Let $w$ be a word in $z_{1}, z_{2}$, and the elements of $H$, which we would like to prove is the identity element of $G$. Since $z_{1}$ and $z_{2}$ 
centralize $K_{2}$, we can determine from the elements of $H$ appearing in the word $w$ a subgroup $K_{0}$ of $K_{2}$ which centralizes $w$. Assuming that $\left|K_{2}: K_{0}\right|$ is not too large, it is possible to find representatives for each of the orbits of $K_{0}$ in $\Omega$. Since $w$ centralizes $K_{0}$, to prove that $w=1$, it therefore suffices to show that $w$ fixes each of these representatives.

Finally as to (D), how does one go about making the right choices for the ultimate generators of $G$ ? Since one begins with a subgroup $H$ of $G$, which is assumed to be known, one has a set of generators for $H$ (and as remarked, an actual presentation), so it is the additional generators that we are talking about. In the case of the baby monster, the group $G$ is by definition generated by a certain conjugacy class of involutions with very special properties, so many relations were known to hold and these largely forced the definition of the permutations $z_{1}$ and $z_{2}$. On the other hand, in the construction of $L y$ and $O N$ a process of trial and error was required.

In fact, in the case of a group $G$ of type $O N$, in order to simplify the calculations, Sims assumed that $G$ possessed an outer automorphism $\alpha$ of period 2 and then constructed the extended group $G\langle\alpha\rangle$. Hence Sims proved the existence and uniqueness of a simple group of type $O N$ which admits an automorphism of period 2. Thus, although unlikely, there conceivably exists another simple group of type $O N$, not possessing such an outer automorphism. In other words, the uniqueness problem for $O N$ is not yet settled. However, a student of Sims is presently working on this question and we hope it will be resolved well within a year's time."

The remarkable Thompson group $F_{3}$ is constructed from a certain 248dimensional lattice associated with the complex Lie group $E_{8}$ (which possesses a representation of this degree) [205]. Although some computer checking (carried out by Peter Smith) is required for its construction, we prefer to discuss it in $\$ 8$ along with the Conway groups and the Leech lattices.

Harada's group $F_{5}$, although not a rank 3 permutation group, is constructed by the same general method that Conway and Wales used to construct the rank 3 Rudvalis group (see the next section) [44]. Their construction was made from a certain 28-dimensional complex representation, whereas $F_{5}$ is constructed from a 133-dimensional representation. It was S. Norton [230] who actually carried out this construction, on the basis of the group-theoretic information provided by Harada. Both groups $R u$ and $F_{5}$ require some computer calculations: McKay and Landauer carrying these out for Rudvalis' group and P. Smith for Harada's group. We shall therefore say nothing further about the construction of $F_{5}$.

Summarizing the results of this section (omitting $J_{2}, M(22), M(23), M(24)$, and $F_{3}$, which are to be discussed later), we have

THEOREM 5. There exists a unique (simple) group of each of the types $\mathrm{J}_{3}, \mathrm{He}$, $L y$, and $F_{2}$; and there exists a unique group of type $O N$ which possesses an outer automorphism of period 2. ${ }^{11}$

6. Sporadic groups as rank 3 permutation groups. It was known that the classical groups have primitive rank 3 representations as permutation groups:

\footnotetext{
"ADDED IN Proor. This work has now been completed. Thus Andrelli and Sims have shown that there exists a unique simple group of type $O N$.
} 
The linear groups acting on the set of lines of projective space, the symplectic and unitary groups acting on the set of absolute points, and the orthogonal groups on the set of singular points. These representations are all clearly related to their structure as groups of Lie type, since the one-point stabilizers are parabolic subgroups in each case. $E_{6}$ has a similar such rank 3 representation on the cosets of a suitable parabolic. Likewise any quadruply transitive group (hence the alternating, symmetric, and Mathieu groups) have such a representation, when considered as permutation groups on the set of unordered pairs of distinct letters. In addition, Wielandt has shown that a primitive permutation group of degree $2 p, p$ a prime, is either doubly transitive or of rank 3 [218], [219].

Thus these primitive rank 3 groups constitute an important class of permutation groups, which had been studied first by Wielandt and then in the 1960s by D. Higman [114]. But until Janko's group $J_{2}$ appeared on the scene [131], no new simple groups had ever been found through any of these investigations. However, with the discovery of $J_{2}$, there followed in rapid succession the construction of four more primitive rank 3 permutation groups: McLaughlin's group $M c$, the D. Higman-Sims group $H S$, the Suzuki (sporadic) group $S u z$, and the Rudvalis group $R u$. Furthermore, Fischer's first three sporadic groups $M(22), M(23), M(24)$, which arose from his analysis of groups generated by a conjugacy class of "3-transpositions" (to be described in the next section) were each constructed from a rank 3 permutation representation.

The table below lists each group, its one-point stabilizer, its degree (the number of cosets of the one-point stabilizer), and the sizes of the two nontrivial orbits of the one-point stabilizer, which are referred to as the subdegrees.

\begin{tabular}{ccccl} 
group & one-point stabilizer & degree & \multicolumn{2}{c}{ subdegrees } \\
\hline$J_{2}$ & $U_{3}(3)$ & 100 & 36 & 63 \\
$H S$ & $M_{22}$ & 100 & 22 & 77 \\
$M c$ & $U_{4}(3)$ & 275 & 112 & 162 \\
$S u z$ & $G_{2}(4)$ & 1782 & 416 & 1365 \\
$R u$ & ${ }^{2} F_{4}(2)$ & 4060 & 1755 & 2304 \\
$M(22)$ & $U_{6}(2)$ & 3510 & 693 & 2816 \\
$M(23)$ & $M(22)$ & 31,671 & 3510 & 28,160 \\
$M(24)$ & $Z_{2} \times M(23)$ & 306,936 & 31,671 & 275,264 \\
$M(24)^{\prime}$ & $M(23)$ & 306,936 & 31,671 & 275,264
\end{tabular}

The group $M(24)$ is not simple, but has a simple subgroup $M(24)^{\prime}$ of index 2; and the intersection of the one-point stabilizer with $M(24)^{\prime}$ is $M(23)$.

The first method of studying rank 3 groups was in terms of an associated combinatorial block design and certain related incidence matrices, the general theory being developed by $\mathrm{D}$. Higman, who characterized some of the classical groups from this point of view. The initial construction of $J_{2}$ by $\mathrm{M}$. Hall and Wales was carried out along these lines [103]. However, this point of view has for the most part been superseded by the use of a natural graph 
associated with any transitive permutation group, an idea first introduced and exploited by Sims [171].

Definition 1. Let $G$ be a transitive permutation group on a set $\Omega$. For each $a \in \Omega$, let $G_{a}$ denote the subgroup of $G$ fixing $a$, and let $\Delta(a)$ be one of the remaining orbits of $G_{a}$ on $\Omega-\{a\}$, chosen so that for $a, b \in \Omega$, if $b=a^{g}$, then $\Delta(b)=(\Delta(a))^{g}$. In other words, beginning with a fixed $a \in \Omega$, and fixed orbit $\Delta(a)$, we consider the translates of $(a, \Delta(a))$ under the action of $G$.

We now define a directed graph $\Gamma$ as follows. The vertices of $\Gamma$ are the elements of $\Omega$, so that $|\Gamma|=|\Omega|$. By definition, the vertex $a \in \Gamma$ is connected by an edge of $\Gamma$ to the vertex $b \in \Gamma$ if and only if $b \in \Delta(a)$.

It is clear from this definition that the action of $G$ on $\Omega$ induces a transitive action of $G$ on the vertices of $\Gamma$, which transforms edges into edges. We say that $G$ is a (transitive) group of automorphisms of $\Gamma$. We define Aut $(\Gamma)$ to be the group of all edge-preserving permutations of $\Gamma$.

All this is fine if we have a transitive permutation group $G$ to begin with. However, our problem is rather to construct such a group $G$, given a prospective one-point stabilizer $H$, degree, and a permutation representation of $H$ on $\Omega-\{a\}$. Such a group $G$ is called a transitive extension of $H$. Usually we impose additional internal restrictions on $G$. We have seen how difficult such construction can be in the previous section. Is it therefore likely that the process can be simplified by turning the problem into the construction of a graph having a transitive automorphism group? Well, this is indeed the case when the proposed group has rank 3. In that case, one has $a$, $\Delta(a)$, and a single additional orbit $\Phi(a)$; and from the internal information, one can "see" which points of $\Delta(a)$ and $\Phi(a)$ and which pairs of points of $\Delta(a), \Phi(a)$, respectively, to connect by an edge in order that the resulting graph possess an automorphism moving $a$ (which is the precise requirement for transitivity). Except for the Rudvalis group, it has been possible to carry out the construction of the appropriate graph and the proof of transitivity of its automorphism group entirely by hand.

For example, Higman and Sims began with the Steiner triple system $S(3,6,22)$ associated with $M_{22}$ and defined the vertices of their graph $\Gamma$ to be

$$
\{*\} \cup \Omega \cup \Lambda \text {, }
$$

where $\{*\}$ is a new symbol, $\Omega$ is the set of 22 points of $S(3,6,22)$ and $\Lambda$ is the set of 77 hexads of $S(3,6,22)$. To define $\Gamma$, connect $\{*\}$ to each point of $\Omega$, connect each point of $\Omega$ to those hexads of $\Lambda$ which contain it, and connect two hexads of $\Lambda$ if they are disjoint.

Higman and Sims proved that $\operatorname{Aut}(\Gamma)$ is a transitive group having a simple subgroup of index 2 and order 44,352,000-a new sporadic group [115]. Their construction was carried out in the course of a single 24-hour period, following a lecture by M. Hall at Oxford on the group $J_{2} ! !$

It is certainly not obvious that $\Gamma$ possesses an automorphism moving $\{*\}$. However, there is a more geometric description of the Steiner system $S=$ $S(3,6,22)$ in which the existence of such an automorphism becomes almost transparent.

Indeed, choose a point of $\Omega$, which we denote by $\infty$. Then $M_{21}\left(\cong L_{3}(4)\right)$ is the subgroup of $M_{22}$ fixing $\infty$. The $21=4^{2}+4+1$ points of $\Omega-\{\infty\}$ can 
therefore be identified with the projective plane $\mathscr{P}=\mathscr{P}_{2}(4)$ over $G F(4)$ in such a way that $M_{21}$ acts on $\mathscr{P}$.

Furthermore, $M_{21}$ has two orbits $\Lambda_{1}$ and $\Lambda_{2}$ in its action on $\Lambda$, with $\left|\Lambda_{1}\right|=21$ and $\left|\Lambda_{2}\right|=56$. In terms of the projective plane $\mathcal{P}$, one has the following description of $\Lambda_{1}$ and $\Lambda_{2}$.

(a) If $B \in \Lambda_{1}$, then $B=\{\infty\} \cup L$, where $L$ consists of the five points of a line of $\mathscr{P}$. (Note that $\mathscr{P}$ has exactly 21 distinct lines.)

(b) If $B \in \Lambda_{2}$, then $B$ consists of six points of $\mathcal{P}$, no three collinear.

There are actually $3 \cdot 56$ sets of six points of $\mathscr{P}$, no three collinear, the full projective group $P G L(3,4)$ in its natural action on $\mathcal{P}$ permuting this set $\theta$ of 168 hexads transitively. However, the "little" projective group $L_{3}(4)$ has three orbits $\theta_{1}, \theta_{2}, \theta_{3}$ on $\theta$ and $\Lambda_{2}=\theta_{i}$ for some $i, 1 \leqslant i \leqslant 3$.

Now the transpose-inverse map $\alpha$ of $\operatorname{PGL}(3,4)$ can be realized as a "polarity" of $\mathcal{P}$-i.e., a one-one transformation of points to lines and lines to points which preserves the incidence relation and has period 2. The remarkable fact in this particular case is that $\alpha$ induces a permutation (which we denote by the same letter) of the set $\theta$. Indeed, any $C \in \mathcal{O}$ is taken by $\alpha$ into 6 lines of $\mathscr{P}$ (no three copunctal), which intersect in exactly 15 points. The complementary set $D$ of 6 points of $\mathscr{P}$ has the property that no three are collinear, so $D \in \mathcal{O}$. One now defines

$$
\alpha(C)=D \text {. }
$$

Since $\alpha$ normalizes the group $L_{3}(4)$, we see that $\alpha$ transforms the orbits $\theta_{1}$, $\hat{\theta}_{2}, \theta_{3}$ among themselves. But $\alpha$ has period 2, so it leaves at least one $\hat{\theta}_{i}$ invariant. Thus without loss, we can assume that $\alpha$ leaves $\Lambda_{2}$ invariant.

Furthermore, this polarity $\alpha$ induces a permutation of the set $\mathscr{P} \cup \Lambda_{1}=(\Omega$ $-\{\infty\}) \cup \Lambda_{1}$, which interchanges the subsets $\Omega-\{\infty\}$ and $\Lambda_{1}$ (again denoted by the same letter): namely, if $\alpha$ interchanges the point $a \in \mathscr{P}=\Omega-$ $\{\infty\}$ and the line $L$ of $\mathscr{P}$, define

$$
\alpha(a)=\{\infty\} \cup L \text { and } \alpha(\{\infty\} \cup L)=a .
$$

Thus $\alpha$ is defined on the 98 points $(\Omega-\{\infty\}) \cup \Lambda$ of $\Gamma$. One extends $\alpha$ to a map of $\Gamma$ by defining

$$
\alpha(\{*\})=\infty \text { and } \alpha(\infty)=\{*\}
$$

It is now an easy matter to check directly that $\alpha$ preserves the incidence relations of the graph and so is an automorphism of $\Gamma$ which moves the point $\{*\}$.

Suzuki has described a lovely construction of a sequence of graphs, which produce larger rank 3 permutation groups from smaller ones, beginning with $\Sigma_{4}$ and ending with the Suzuki sporadic group, and on the way picking up $J_{2}$ [192].

Let $H$ be a transitive permutation group and let $\Gamma_{0}$ be an associated graph. Construct a graph $\Gamma$ with vertices

$$
\{*\} \cup \Gamma_{0} \cup \Lambda,
$$


where $\{*\}$ is a new symbol and $\Lambda$ denotes the set of involutions of $H$. Connect $\{*\}$ to each point of $\Gamma_{0}$, connect two points of $\Gamma_{0}$ if they are connected as points in the graph of $H$, connect a point $a$ of $\Gamma_{0}$ to an involution $b$ in $\Lambda$, if $b \in H_{a}$, the subgroup of $H$ fixing $a$, and connect two involutions $a, b \in \Lambda$ if $a$ and $b$ do not commute, but there is a third involution $c \in \Lambda$ centralizing each of them.

Beginning with $\Sigma_{4}$ and its natural graph with four points and no edges $\Gamma_{0}$, one obtains a graph $\Gamma_{1}$ of order 14 with $\operatorname{Aut}\left(\Gamma_{1}\right) \cong P G L(2,7)$. If one repeats the process with $H$ as $P G L(2,7)$ and with $\Gamma_{1}$ as $\Gamma_{0}$, one obtains a graph $\Gamma_{2}$ of order 36 with $\operatorname{Aut}\left(\Gamma_{2}\right) \cong G_{2}(2)$. Continuing the process as long as one can, one obtains the following graphs and automorphism groups; (the final construction leading to $\operatorname{Aut}(\mathrm{Suz})$ requires a slight modification of the procedure, with $\Lambda$ consisting of suitable four groups rather than involutions of $H$ ):

\begin{tabular}{ccc} 
graph & order & Automorphism group \\
\hline$\Gamma_{3}$ & 100 & $\operatorname{Aut}\left(J_{2}\right)$ \\
$\Gamma_{4}$ & 416 & $\operatorname{Aut}\left(G_{2}(4)\right)$ \\
$\Gamma_{5}$ & 1782 & $\operatorname{Aut}(\mathrm{Suz})$
\end{tabular}

Unfortunately this process collapses if one tries to repeat it with Aut(Suz) as $H$ and $\Gamma_{5}$ and $\Gamma_{0}$. We note also that $J_{2}$ and $S u z$ are of index 2 in their automorphism groups.

McLaughlin's construction of his graph and group was carried out along similar lines [147]; however, Conway and Wales' construction of the Rudvalis group was trickier. Griess had shown that if $R u$ existed, it must have a nontrivial cover $\widehat{R u}$ by $Z_{2}$. Rudvalis gave evidence that $\widehat{R u}$ would have to possess a 28-dimensional complex representation [163] and then Feit proved that if $R u$ existed, $\widehat{R u}$ would indeed have a representation of this degree. Conway and Wales proceeded from this presumed representation and their argument involved the determination of a set of 4060 quadruples of vectors $(v, i v,-v,-i v)$ in complex 28-space, whose automorphism group is transitive of rank 3 as a permutation group on these 4060 quadruples. Moreover, the stabilizer of a quadruple is an extension of ${ }^{2} F_{4}(2)$ by $Z_{2}$. Thus they constructed $\widehat{R u}$. The group $R u(=\widehat{R u} /$ center $)$ can be viewed as a permutation group of the corresponding 4060 one-dimensional subspaces.

As for the Fischer groups, their graph is intimately related to the conjugacy class of involutions which define them and will be described in the next section.

So much then for existence; what about the question of the uniqueness of these groups? Since each is determined from the automorphism group of a graph, the question clearly reduces to the uniqueness of the graph. However, the specified action of the one-point stabilizer $H$ on each orbit is such a strong restriction that it forces in each case only one possibility for the graph of a transitive extension of $H$ in which $H$ has the given action.

Finally, defining groups of type $H S, M c, S u z$, and $R u$ by the conditions of the table, we can summarize the above discussion as follows:

Theorem 2. There exists a unique (simple) group of type $\mathrm{J}_{2}, \mathrm{HS}, \mathrm{Mc}, \mathrm{Suz}$, and $R u$. 
7. Transpositions and the Fischer groups. To the nonexpert, the name of Bernd Fischer is known solely for its connection with a number of sporadic simple groups, but to the practitioner, he is recognized as the developer of internal geometric analysis, an approach to finite group theory which has not only given rise to five new simple groups, but supplemented by the work of his disciple Timmesfeld, has provided a fundamental general technique for studying simple groups.

From the outset of his career, Fischer has been interested in the generation of a group from a conjugacy class of its involutions by conditions on the order of the product of two elements of the class. The underlying motivation here is the example of the symmetric groups, which are generated by their transpositions, which form a single conjugacy class and have the property that the product of any two has order 1,2 , or 3 . (If $a, b$ are two such transpositions, then $|a b|=1$ if $a=b,|a b|=2$ if $a$ centralizes $b$ and $a \neq b$, and $|a b|=3$ if $a$ and $b$ do not commute, in which case $a b$ is a 3-cycle.)

If $x, y$ are any two involutions of a group $G$, then $x y$ is inverted by $x$ and consequently the group $\langle x, y\rangle=\langle x, x y\rangle$ is always a dihedral group of order $2|x y|$. Hence for pairs of involutions, a condition on the order of $\langle x y\rangle$ can be equivalently expressed in terms of the group $\langle x, y\rangle$. We note, however, that from a more general perspective (i.e., in cases when $x, y$ are not necessarily involutions), it is the group $\langle x, y\rangle$ rather than $|x y|$ which is the relevant object of interest.

From his considerations, Fischer was led to make the following definition.

Definition 1 . A conjugacy class $D$ of involutions of a group $G$ is said to be a class of $p$-transpositions, $p$ an odd prime, provided the product of any two elements of the class $D$ has order 1,2 , or $p$.

Fischer investigated groups which were generated by a class of $p$-transformations and his first classification [59] foreshadowed the major achievements to come.

THEOREM 1 . Let $G$ be a group generated by a class of p-transpositions $D$ and assume the following conditions hold:

(a) Any three pairwise distinct elements of $D$ do not generate a 2-group; and

(b) If $x \in D$, then $C_{D}(x) \neq x$.

Then $G \cong \Sigma_{4}$ or $\Sigma_{5}$. In particular, $p=3$.

The proof of this result made use of the following earlier result of Fischer's, which he established by transforming the problem into a question about distributive quasigroups [60]. This was a highly original way of dealing with a purely group-theoretic question.

THEOREM 2. Let $G$ be a group which is generated by a conjugacy class of involutions $D$ and suppose that whenever $x, y \in D$ with $x \neq y$, the order of $x y$ is a power of a fixed odd prime $p$. Then $G^{\prime}$ is nilpotent.

Note that the hypotheses imply that distinct elements of $D$ never commute.

Using his result on quasigroups, Fischer similarly proved.

THEOREM 3. Let $G$ be a group which is generated by a conjugacy class of involutions $D$ such that distinct elements of $D$ do not commute. Assume that for all $x \in D$, 


$$
C_{x}=O_{2}\left(C_{x}\right) \times O\left(C_{x}\right) .
$$

Then $G$ is solvable.

(We remark that Theorems 2 and 3 can now be derived from the odd order theorem and Glauberman's $Z$ *-theorem (Theorem IV.5.7 below).)

This last result was used by Fischer to prove the solvability of a finite group $G$ admitting a fixed-point-free automorphism $\alpha$ of order $2 p, p$ an odd prime (with some restriction on $C_{G}\left(\alpha^{p}\right)$ ) [61].

These results were just "warming up" exercises for Fischer, in preparation for the main event, which consisted of the following magnificent theorem [62], [63].

THEOREM 4. Let $G$ be a group with $Z(G)=1$ and $G^{\prime}=\left(G^{\prime}\right)^{\prime}$, which is generated by a conjugacy class of 3-transpositions. Then one of the following holds:

(i) $G \cong \Sigma_{n}$ for some $n$ and except when $n=6, D$ is the set of transpositions;

(ii) $G \cong S p(2 n, 2)$ and except when $n=2, D$ is the set of symplectic transvections;

(iii) $G \cong O_{2 n}^{ \pm}(2)$, and $D$ is the set of transvections leaving the corresponding quadratic form invariant;

(iv) $G \cong U_{n}(2)$ and $D$ is the set of unitary transvections;

(v) $G \cong O_{n}^{ \pm}(3)\langle d\rangle$, where $d \in D$ and given $\pi= \pm 1, D$ is the set of reflections: $x \mapsto x+\pi(x, a) a$, where $a$ is a vector such that the inner product $(a, a)=\pi ;$ or

(vi) $G$ is one of three new finite groups, denoted by $M(22), M(23)$, and $M(24)$, and $D$ is uniquely determined for each group.

The exceptions in (i) and (ii) are due to the fact that $\Sigma_{6} \cong S p(4,2) \cong$ $O_{4}^{-}(3)\langle d\rangle$. Note that the hypothesis $G^{\prime}=\left(G^{\prime}\right)^{\prime}$ implies that $G$ is nonsolvable.

The theorem clearly suggests that the given hypothesis must be closely connected with some natural geometric problem, the various solutions of which lead to the alternatives of the theorem, the groups $M(22), M(23)$, $M(24)$ arising as exceptional cases in roughly the same way as $G_{2}, F_{4}, E_{6}, E_{7}$, $E_{8}$ occur in the classification of simple Lie algebras. Thus the method of attack must be to build a geometry out of the class of 3-transpositions on which the group $G$ will act as a group of automorphisms; and then from the properties of these geometries, to determine the possibilities for $G$.

I would like to describe some of the central steps involved in the proof. Denote by $E$ a set of pairwise commuting involutions of $D$ of maximum size $n$. Fischer proves

Proposition 5. (i) $N_{G}(E)$ contains a Sylow 2-subgroup of $G$;

(ii) $N_{G}(E)$ acts doubly transitively on the set $E$; and

(iii) $N_{G}(E) / C_{G}(E)$ is isomorphic to one of the following groups: $\Sigma_{n}, A_{n}$, $G L(n, 2), L_{m}(4)$, where $m=[n / 2], \Sigma_{2^{n}} \cdot G L(n, 2), M_{22}, M_{23}$, or $M_{24}$.

It is the last three possibilities in (iii) which give rise to the Fischer groups and will explain why he adopted the notation $M(22), M(23), M(24)$ for them.

Proposition 6. For $x \in D$, set $D_{x}=C_{D}(x)-\{x\}$. Then $D_{x}$ is a class of 
conjugate elements of $\left\langle D_{x}\right\rangle$. In particular, $\left\langle D_{x}\right\rangle \leqslant C_{G}(x)$ acts transitively on the set $D_{x}$.

This remarkable fact allows Fischer to use induction in the analysis. However, there are cases in which $\left\langle D_{x}\right\rangle$ has a nontrivial center or is solvable, so these must be analyzed separately.

Proposition 7. (i) If $O\left(\left\langle D_{x}\right\rangle\right) \nless Z\left(\left\langle D_{x}\right\rangle\right)$, then $G \cong \Sigma_{5}$;

(ii) If $O_{2}\left(\left\langle D_{x}\right\rangle\right) \nless Z\left(\left\langle D_{x}\right\rangle\right)$, then $G \cong S p(n, 2)$ or $U_{n}(2)$; and

(iii) If $\left\langle D_{x}\right\rangle$ is solvable, then $G \cong \Sigma_{5}, \Sigma_{6}, U_{4}(2)$, or $U_{5}(2)$.

The following result is fundamental and reveals the geometry that lies at the heart of this class of groups.

Proposition 8. For $x \in D$, set $F_{x}=D-\left(D_{x} \cup\{x\}\right)$. Then we have

(i) $\left\langle D_{x}\right\rangle$ acts transitively on $F_{x}$; and

(ii) As a permutation of $D, G$ has rank 3 with one-point stabilizer $\left\langle D_{x}\right\rangle=$ $C_{G}(x)$ having orbits $\{x\}, E_{x}$, and $F_{x}$.

Thus the geometry underlying $G$ is that of a graph associated with a rank 3 permutation group whose vertices can be identified with the elements of $D$, a given vertex $x \in D$ being connected to those elements of $D-\{x\}$ with which it commutes. As we have pointed out in the previous section, the symmetric, alternating, unitary, symplectic, and orthogonal groups all have natural representations as automorphism groups of such graphs. What Fischer has done is to construct these classical graphs (as well as new ones for $M(22), M(23), M(24))$ from internal properties of $G$ which follow from the existence of the class of generating 3-transpositions. The graphs associated with $D$ are, in general, of a special type, which Fischer calls triple-graphs and the analysis is reduced to the study of these triple graphs and their so-called triple-maps. (We shall not attempt to define these terms here.)

In the previous section, we have also listed the subdegrees, and one-point stabilizers of $M(22), M(23), M(24)$.

At this point, Aschbacher established a beautiful extension of Fischer's theorem [7]. To state it, we need the following definition.

Definition 9. A conjugacy class $D$ of involutions of a group $G$ is said to be a class of odd transpositions if the product of any two noncommuting elements of $D$ has odd order.

THEOREM 10. Let $G$ be a group such that $\operatorname{Sol}(G)=1$ and $G^{\prime}=\left(G^{\prime}\right)^{\prime}$, which is generated by a conjugacy class $D$ of odd transpositions. Then one of the following holds:

(i) $D$ is a class of 3-transpositions (whence $G$ is determined from Fischer's theorem);

(ii) $G \cong S p(n, q), U_{n}(q)$, or $O_{n}^{ \pm}(q)$, where $q=2^{m}$ for some $m$, and $D$ is a class of transvections;

(iii) $G \cong$ an extension of $O_{n}^{ \pm}(5)$ by $Z_{2}$ and $D$ is a class of orthogonal reflections;

(iv) $G \cong S z(q), q=2^{m}$, modd, $m>1$; or

(v) $G \cong L_{2}(q) / \Sigma_{n}, q=2^{m}, m>1$. 
Thus Aschbacher's theorem includes all symplectic, unitary, and orthogonal groups of characteristic 2, whereas Fischer's hypothesis picks up only those over the prime field $G F(2)$.

Fischer showed that his groups were all rank 3 as permutation groups on the set of 3-transpositions. In the course of proving his main theorem, Aschbacher derives a converse of this result [7].

THEOREM 11. Let $G$ be a group with $\operatorname{Sol}(G)=1$ which is generated by a conjugacy class $D$ of involutions. If $G$ has rank 3 as a permutation group on the set $D$, then $D$ is a class of 3-transpositions of $G$.

As would be expected, Aschbacher's proof of Theorem 10 utilizes many of the concepts introduced by Fischer; but if anything, it is even more geometric than the 3-transposition theorem. The action of $G$ on the geometry determined by the class $D$ and, in the case of the situations giving rise to the classical groups, the associated bilinear form preserved by $G$ dominates the analysis. For example, to identify the symplectic groups, Aschbacher invokes a theorem of Dembowski and Wagner which characterizes projective space among "symmetric block designs" [48] to conclude that $G$ is acting on projective $n$-space over $G F(q), n$ odd, $q=2^{m}$. Since he also knows in this case that $D$ is the set of nontrivial "elations" commuting with a certain "symplectic polarity", he is then able to assert that $G=\langle D\rangle \cong S p_{n+1}(q)$.

In cases in which the action of $G$ on the set $D$ has rank 3 -and, in particular, in the proof of Theorem 11-Aschbacher makes strong use of various arithmetical relations which D. Higman established for arbitrary rank 3 permutation groups.

The next stage in the analysis of groups generated by a class $D$ of involutions allows products of pairs of elements of $D$ to have order divisible by 4. A theorem of Baer and Suzuki [84, Theorem 3.8.2], disposes of a trivial case.

THEOREM 12. If $D$ is a conjugacy class of elements of prime order $p$ in a group $G$ with the property that $\langle x, y\rangle$ is a p-group for every pair $x, y \in D$, then $D \leqslant O_{p}(G)$.

Thus as a corollary, one has

CoRollary 13. If a group $G$ is generated by a conjugacy class $D$ of involutions such that the product of any two elements of $D$ has order a power of 2 , then $G$ is a 2-group.

Hence the first interesting case is incorporated in the following definition.

Definition 14. A conjugacy class $D$ of involutions of a group $G$ is said to be a class of $\{3,4\}$-transpositions if the product of any two elements of $D$ has order $1,2,3$, or 4 . In order to exclude the case of 3-transpositions, we say that $D$ is nondegenerate if some product of elements of $D$ has order 4 . Furthermore, we say that $D$ is a class of $\{3,4\}^{+}$-transpositions provided whenever $x$, $y \in D$ and $|x y|=4$, then $(x y)^{2} \in D$.

A natural example is the group $G L(n, 2)$, which is generated by its transvections (involutions acting trivially on a hyperplane in the natural 
representation), these transvections forming a conjugacy class of $\{3,4\}^{+}$. transpositions.

Timmesfeld began his investigations with an analysis of groups generated by a nondegenerate class of $\{3,4\}^{+}$-transpositions and obtained the following lovely classification theorem [207], [208].

THEOREM 15. Let $G$ be a group with $Z(G)=1$ and $O(G)=1$ which is generated by a nondegenerate class of $\{3,4\}^{+}$-transpositions. Then one of the following holds:

(i) $G \cong G L(n, 2), n \geqslant 3$;

(ii) $G \cong S p(2 n, 2), n \geqslant 3$;

(iii) $G \cong O_{2 n}^{ \pm}(2), n \geqslant 4$; or

(iv) $G \cong G_{2}(2)^{\prime},{ }^{3} D_{4}(2), F_{4}(2),{ }^{2} E_{6}(2), E_{6}(2), E_{7}(2)$, or $E_{8}(2)$.

Moreover, in each case $D$ is a uniquely determined class of 2-central involutions of $G$.

Timmesfeld's analysis is again much in the spirit of Fischer's and Aschbacher's. In the special case that leads to $G L(n, 2)$, using internal properties of $G$ which follow from the existence of the class $D$, Timmesfeld constructs a vector space over $G F(2)$ on which $G$ acts in a prescribed fashion. He actually considers a slightly more general case, which leads to the groups $G_{2}(2)^{\prime}$, and ${ }^{3} D_{4}(2)$ as well as $G L(n, 2)$, the precise hypothesis being that $D$ possesses a nontrivial proper subset $E$ such that

$$
E \cap E^{x}=\varnothing \text { or } E \text { for all } x \in D \text {. }
$$

To obtain the groups $\operatorname{Sp}(2 n, 2)$ and $O_{2 n}^{ \pm}(2)$, Timmesfeld shows under suitable conditions that $G$ is also generated by a class of 3-transpositions, so that the groups can be identified from Fischer's classification theorem. The cases leading to $F_{4}(2),{ }^{2} E_{6}(2), E_{6}(2), E_{7}(2)$, and $E_{8}(2)$ depend upon a set of conditions on the class $D$ which suffice to imply that the multiplication table of $G$ is uniquely determined.

The next step in Timmesfeld's program was to weaken his assumption on the class $D$ by replacing "3" by "odd". Thus we have the following definition.

Definition 16. A conjugacy class $D$ of involutions of a group $G$ is said to be a class of $\{\text { odd }, 4\}^{+}$-transpositions if the product of any two elements $x, y$ of $D$ has order $1,2,4$, or $k$, where $k$ is odd, with the additional restriction that $(x y)^{2} \in D$ whenever $|x y|=4$. Again $D$ is called nondegenerate if some product of elements of $D$ has order 4.

This is a fundamental notion, since every group of Lie type of characteristic 2 (apart from the Ree groups ${ }^{2} F_{4}\left(2^{n}\right)$ ) is generated by a class $D$ of \{odd, 4$\}^{+}$-transpositions, the elements of $D$ being so-called "root involutions". For this reason, Timmesfeld refers to a class of $\{\text { odd, } 4\}^{+}$. transpositions as a class of root involutions.

Timmesfeld's major "root involution" theorem gives a classification of all groups generated by a nondegenerate class of root involutions, the degenerate case being covered by Aschbacher's and Fischer's prior work [208].

THEOREM 17. Let $G$ be a group with $Z(G)=1$ and $O(G)=1$ which is generated by a nondegenerate class of root involutions. Then one of the following holds: 
(i) $G \in \operatorname{Chev}(2)$ (with $G \nRightarrow U_{n}\left(2^{m}\right)$ or $S p\left(4,2^{m}\right)$ ); or

(ii) $G \cong A_{6}$ or $J_{2}$.

Moreover, in (i), either $D$ is the uniquely determined class of root elements corresponding to the long roots of the associated Lie algebra of $G$ or $G \cong$ $F_{4}\left(2^{m}\right)$, in which case $D$ may also be the class of root elements corresponding to the short roots.

The proof is again similar to those of the preceding theorems, in that Timmesfeld must build up the internal structure of $G$ from properties of the class of $D$. The "root subgroups" play an important role in this construction. For $x \in D$, set

$$
D_{x}=\left\{y \in D \mid C_{D}(y)=C_{D}(x)\right\} \text { and } E_{x}=D_{x} \cup\{1\} .
$$

Then $E_{x}$ is called a root subgroup of $G$. Clearly $E_{x}$ is determined independently of the choice of $y \in D_{x}$.

Fix $E_{x}$ and let $\Sigma$ be the conjugacy class of $E_{x}$ in $G$. Then $\Sigma$ is called a class of root subgroups of $G$.

Timmesfeld proves the following result.

Proposition 18. If $\Sigma$ is a class of root subgroups of $G$, then $\left|E_{x}\right|=q=2^{n}$ for some $n$ for each $E_{x} \in \Sigma$. Moreover, if $E_{x}, E_{y} \in \Sigma$, then one of the following holds:

(i) $\left\langle E_{x}, E_{y}\right\rangle$ is elementary abelian;

(ii) $\left\langle E_{x}, E_{y}\right\rangle$ is special of order $q^{3}$ with center of order $q$ and $\left[E_{x}, E_{y}\right] \in \Sigma$; or

(iii) $\left\langle E_{x}, E_{y}\right\rangle \cong L_{2}(q)$.

This result is analogous to that proved by Thompson for odd primes in his study of so-called "quadratic pairs" (see \$4.6).

The case $q=2$ of the root involution theorem is essentially covered by the $\{3,4\}^{+}$-transposition theorem, so Timmesfeld can assume $q>2$ at critical points in the argument. The main idea of the proof is to construct a certain graph $\Gamma(\Sigma)$ whose vertices are the elements of $\Sigma$ and then to prove that $\Gamma(\Sigma)$ is isomorphic to the corresponding graph $\Gamma\left(\Sigma^{*}\right)$ of a suitable group $G^{*}$ of Lie type of characteristic 2. To obtain this isomorphism, Timmesfeld proves a "graph extension" theorem, which asserts that a certain collection of "local" isomorphisms of $\Gamma(\Sigma)$ on $\Gamma\left(\Sigma^{*}\right)$ can be extended to an actual isomorphism of $\Gamma(\Sigma)$ on $\Gamma\left(\Sigma^{*}\right)$. He also argues that the group $G^{*}$ is uniquely determined by its graph and is thus able to conclude that $G \cong G^{*}$.

While Timmesfeld was investigating $\{3,4\}^{+}$-transposition and root involution groups, Fischer was looking at the broader class of $\{3,4\}$-transposition groups. Fischer had developed a general method for attempting to form, from a class $E$ of transpositions of a group $H$, a larger group $G$ containing $H$, generated by a class $D$ of transpositions containing $E$, which can be viewed as an analogue of the construction of a transitive extension with a given one-point stabilizer. The passage from $U_{6}(2)$ to $M(22)$, from $M(22)$ to $M(23)$, and from $Z_{2} \times M(23)$ to $M(24)$ are examples in which the extended groups exist.

Now Fischer knew that his group $\operatorname{Aut}(M(22))$ (a split extension of $M(22)$ by $Z_{2}$ ) was generated by a class $E$ of $\{3,4\}$-transpositions (the elements of $E$ necessarily inducing outer automorphisms of $M(22))$. There was also strong 
evidence that $\operatorname{Aut}(M(22))$ occurred as a subgroup of ${ }^{2} \hat{E}_{6}(2) \cdot 2$. This was a natural situation for Fischer to ask whether the latter group could possibly be imbeddable in a larger group $G$ in such a way that the class $E$ extended to a class of generating $\{3,4\}$-transpositions $D$ of $G$. Herein lay the origin of the baby monster! Fischer was eventually able to derive almost complete information about the internal structure of his presumed group $G$; and, in particular, the information which Leon and Sims required for their construction of $G$ [64]. Thus their existence and uniqueness theorem for the baby monster $F_{2}$ yields the following additional fact.

THEOREM 19. The baby monster $F_{2}$ is generated by a class $D$ of $\{3,4\}-$ transpositions such that $C_{F_{2}}(x) \cong{ }^{2} \hat{E}_{6}(2) \cdot 2$ for all $x \in D$.

This is by no means the end of the Fischer story. In $\$ 4.9$ we shall describe the general fusion theorems of Timmesfeld which are consequences of his root involution theorem.

8. The Leech lattice and the Conway groups. Conway [42] constructed his three simple groups from the automorphism group of the remarkable 24dimensional Leech lattice, which had its origins in the study of close sphere packings [138], [139].

Definition 1. A lattice $\Lambda$ of Euclidean $\mathbf{R}^{n}$ space is the set of all integral linear combinations of $n$ linearly independent vectors $w_{1}, w_{2}, \ldots, w_{n}$ of $\mathbf{R}^{n}$ having the property that the inner product $\left(w_{i}, w_{j}\right)$ is an integer for all $i, j$, $1 \leqslant i, j \leqslant n$.

In particular, $\Lambda$ is an abelian group. If $v_{i}, 1 \leqslant i \leqslant n$, is an orthonormal basis of $\mathbf{R}^{n}, \Lambda$ is said to be integral (rational) if the coordinates of each $w_{j}$ as a linear combination of the $v_{i}$ 's are all integers (rational numbers). Moreover, $\Lambda$ is said to be unimodular if the matrix of the change of basis from the $v_{i}$ 's to the $w_{i}$ 's has determinant 1 .

A rational lattice can always be made into an integral lattice by replacing the given basis vectors by suitable scalar multiples of themselves. Of course, if the original lattice is unimodular, this property will be lost when shifting to a corresponding integral lattice. The Leech lattice is, in fact, a rational unimodular lattice. However, in describing its related geometry, it was easier for Conway to work with an associated integral form of the lattice.

We remark that a set of so-called "fundamental roots" of a complex semisimple Lie algebra $\mathcal{L}$ determines a lattice in $\mathbf{R}^{n}$, where $n$ is the dimension of a Cartan subalgebra of $\mathcal{L}$, the so-called "root lattice" of $\mathcal{L}$. These root lattices are, in general, nonrational.

By definition, the automorphism group $\operatorname{Aut}(\Lambda)$ of a lattice $\Lambda$ in $\mathbf{R}^{n}$ is the subgroup of the rotation group of $\mathbf{R}^{n}$ which transforms $\Lambda$ into itself. The elements of $\operatorname{Aut}(\Lambda)$ are called rotations of $\Lambda$.

To describe the Leech lattice, one begins with the Steiner system $S=$ $S(5,8,24)$ on 24 letters $\Omega$, whose automorphism group is $M_{24}$. The set $\Omega^{*}$ of all $2^{24}$ subsets of $\Omega$ can be viewed as a vector space of dimension 24 over $G F(2)$ if $A+B$ is defined to be the symmetric difference $(A-B) \cup(B-$ $A$ ) for $A, B \in \Omega^{*}$ (i.e., $A, B \subseteq \Omega$ ).

Proposition 2. The octads of $S$ span a 12-dimensional subspace $\mathcal{C}$ of $\Omega^{*}$ 
consisting of $\varnothing, \Omega$, the 759 octads of $S$, their 759 complements in $\Omega$, and 2576 sets of cardinality 12 .

We let $\mathcal{C}_{n}$ be the subset of elements of $\mathcal{C}$ of cardinality $n$, so that

$$
\mathcal{C}=\mathcal{C}_{0} \cup \mathcal{C}_{8} \cup \mathcal{C}_{12} \cup \mathcal{C}_{16} \cup \mathcal{C}_{24}
$$

with $\mathcal{C}_{8}$ consisting of the octads of $S$.

Now let $\left\{v_{i} \mid 1 \leqslant i \leqslant 24\right\}$ be an orthonormal basis of $\mathbf{R}^{24}$, the index set being $\Omega$, which we may identify with $\{1,2, \ldots, 24\}$. For any $T \in \Omega^{*}$ and any $m \in \mathbf{Z}$, define

$$
\begin{aligned}
{[T, m]=\{v \mid v} & =\sum x_{i} v_{i} \text {, where } x_{i} \in Z, \sum x_{i}=4 m, \text { and } \\
x_{i} & \left.\equiv m(\bmod 4) \text { if } i \notin T, x_{i} \equiv m+2(\bmod 4) \text { if } i \in T\right\} .
\end{aligned}
$$

For each pair, $T, U \in \Omega^{*}$ and $m, n \in \mathbf{Z}$, one has

$$
[T, m]+[U, n]=[T+U, m+n] \text {. }
$$

Definition 3. Set $\Lambda=\cup[T, m]$, the union over all $T \in \mathcal{C}, m \in \mathbf{Z}$. In view of (3) and the fact that $\mathcal{C}$ is a subspace, $\Lambda$ is an integral lattice. This is the Leech lattice. For reasons that will soon become clear, Conway sets $.0=$ Aut $(\Lambda)$.

Conway analyzes $\Lambda$ and .0 and establishes all the following facts. First of all, for each $T \in \Omega^{*}$, define

$$
v_{T}=\sum_{i \in T} v_{i}
$$

It is immediate from the definitions that the vector $2 v_{T}$ is in $\Lambda$ for each $T \in \mathcal{C}_{8}$.

Proposition 4. (i) $\Lambda$ is spanned by the 759 vectors $2 v_{T}$ with $T \in \mathcal{C}_{8}$ and any one vector of $\Lambda$, all of whose coordinates are odd integers; e.g., the vector $v_{\Omega}-4 v_{24}$.

(ii) The 759 vectors $2 v_{T}, T \in \mathcal{C}_{8}$, span the sublattice [ $\left[\begin{array}{l}\mathrm{C} \\ \text { ] }] \text {. }\end{array}\right.$

(iii) If $v, w \in \Lambda$, then the inner product $(v, w)$ is a multiple of 8 and $(v, v)$ is a multiple of 16.

Here [ $\mathcal{C}, 4 \mathrm{Z}]$ denotes the set of $[T, m]$ taken over all $T \in \mathcal{C}, m \in \mathbf{4 Z}$.

Definition 5. If $(v, v)=16 n$ for $v \in \Lambda$, $v$ is said to be of type $n$. The set of all $v \in \Lambda$ of type $n$ is denoted by $\Lambda_{n}$.

The set $\Lambda_{1}$ is empty, so that $\Lambda_{2}$ consists of the vectors of minimum type. This set plays an important role in the analysis.

Proposition 6. (i) $\Lambda_{2}$ has cardinality 196560.

(ii) If $\Lambda_{2}(v)$ denotes the subset of $\Lambda_{2}$ orthogonal to the vector $v \in \Lambda_{2}$, then $\Lambda_{2}(v)$ has cardinality 93150 .

Observe next that any permutation $\pi$ of $\Omega$ extends to an orthogonal transformation of $\mathbf{R}^{24}$ (denoted by the same letter) under the definition

$$
\left(v_{i}\right) \pi=v_{i \pi} \text { for all } i, 1 \leqslant i \leqslant 24 .
$$

Clearly $\pi$ will be a rotation of $\Lambda$ provided it preserves $\mathcal{C}$. Hence the rotations 
induced from the automorphism group of the Steiner system $S=S(5,8,24)$ determine a subgroup $M \cong M_{24}$ of .0 .

Furthermore, to each $T \in \Omega^{*}$, one can associate an orthogonal reflection $\varepsilon_{T}$ of $\mathbf{R}^{24}$ by setting

$$
\left(v_{i}\right) \varepsilon_{T}=v_{i} \text { or }-v_{i} \quad \text { according as } i \notin T \text { or } i \in T .
$$

Likewise $\varepsilon_{T}$ is a rotation of $\Lambda$ provided $T \in \mathcal{C}$. Moreover, any two such rotations commute and so as $|\mathcal{C}|=2^{12}$, they generate an elementary abelian subgroup $E$ of .0 of order $2^{12}$.

The rotations of $M$, arising as they do from elements of $\operatorname{Aut}(S)$, induce an action on the elements of $E$ and so $M$ normalizes $E$. Thus .0 contains a subgroup

$$
N=E M,
$$

a split extension of $E_{2^{12}}$ by $M_{24}$.

\section{Conway proves}

Proposition 7. $N$ contains the subgroup of .0 fixing any one coordinate vector $v_{i}$.

The crucial result that must be established is the following.

THEOREM 8. $N$ is a proper subgroup of .0 .

Thus Conway must produce rotations of $\Lambda$ which move the coordinate vectors. Indeed, let $T$ be any element of $\Omega^{*}$ of cardinality 4 (a tetrad). From properties of the Steiner system $S, T$ is contained in exactly 5 octads of $S$, which can therefore be expressed as $T+T_{i}, 1 \leqslant i \leqslant 5$, where each $T_{i}$ is a tetrad. Set $T_{0}=T$ and

$$
\Phi(T)=\left\{T_{i} \mid 0 \leqslant i \leqslant 5\right\} .
$$

Since any 5 elements of $\Omega$ lie in a unique octad, it follows that the $T_{i}$ are pairwise disjoint and hence that $\Omega=\cup T_{i}, T_{i} \in \Phi(T)$.

Then for each $j, .1 \leqslant j \leqslant 24, j$ lies in a unique tetrad $T_{i}$ of $\Phi(T)(i$ depending on $j$ ). Define the map $\eta_{T}$ by setting

$$
\left(v_{j}\right) \eta_{T}=v_{j}-\frac{1}{2} v_{T_{i}}, \quad 1 \leqslant j \leqslant 24
$$

Conway shows that for each tetrad $T$, the transformation $\eta_{T} \varepsilon_{T}$ is a rotation of $\Lambda$ and so is an element of .0. Thus $N<.0$, as asserted.

With this information available, Conway is now able to argue:

THEOREM 9. (i) . 0 is transitive on $\Lambda_{2}$.

(ii) If $v \in \Lambda_{2}$ and $w \in \Lambda_{2}(v)$, then the subgroup $.0_{v w}$ of .0 fixing $v$ and $w$ is contained in $N$ and is a split extension of $M_{22}$ by an elementary group of order $2^{10}$.

Note that as .0 consists of orthogonal transformation and .0 leaves $\Lambda_{2}$ invariant,.$_{v}$ leaves $\Lambda_{2}(v)$ invariant for any $v \in \Lambda_{2}$.

Combined with Proposition 6, Conway immediately obtains the following corollary.

THEOREM 10. (i) $|.0|=196560 \cdot 93150 \cdot 2^{10} \cdot\left|M_{22}\right|$.

(ii) $N$ is a maximal subgroup of .0 . 
The group .0 has a center of order 2 , generated by the reflection $\varepsilon_{\Omega}$.

For $v \in \Lambda_{2}$, the pair $\{v,-v\}$ is called a diameter and the set of diameters is denoted by $\bar{\Lambda}_{2}$. Thus $\bar{\Lambda}_{2}$ has cardinality 98280 . Also set .1 $=.0 /\left\langle\varepsilon_{\Omega}\right\rangle$, so that .1 acts on $\bar{\Lambda}_{2}$. The analysis of the action of .1 on $\bar{\Lambda}_{2}$ is a consequence of the following property of .0 .

Proposition 11. .0 acts transitively on the set of ordered pairs of vectors of $\Lambda_{2}$ with any given scalar product.

The number of $w \in \Lambda_{2}$ having scalar product $-32,-16,-8,0,8,16,32$ with a given $v \in \Lambda_{2}$ is

$$
\text { 1, 4600, 47104, 93150, 47104, 4600, 1, }
$$

respectively, and these are the only possible scalar products.

With this information, Conway is able to prove

THEOREM 12. .1 is a simple group (of order $\frac{1}{2}|.0|$ ).

Now define .2 to be the subgroup of .0 fixing any one vector of $\Lambda_{2}$. Conway also proves

THEOREM 13. .2 is a simple group of order $93150 \cdot 2^{10} \cdot\left|M_{22}\right|$.

Conway determines yet a third simple group inside .0.

THEOREM 14. (i) .0 acts transitively on the set $\Lambda_{3}$.

(ii) If .3 denotes the subgroup of .0 fixing any one vector of $\Lambda_{3}$, then .3 is a simple group of order $2^{11} \cdot 3^{7} \cdot 5^{3} \cdot 7 \cdot 11 \cdot 23$.

The groups $.1, .2$, and .3 are sporadic groups, distinct from any of those previously discovered.

As is clearly evident, there is a rich geometry associated with .0 and the Leech lattice. In addition to the groups $M_{22}, M_{23}, M_{24}$, also $M c, H S, J_{2}$, and Suz arise in a natural way as subgroups of .0. For example, .0 acts transitively on $\Lambda_{5}$ and on $\Lambda_{7}$. If .5 and .7 denote the corresponding one-point stabilizers, then $.5 \cong \operatorname{Aut}(M c)$ and $.7 \cong H S$. Furthermore, .0 has an element of order 3 which acts fixed-point-free on $\Lambda$. Its centralizer in .0 is the 3 -fold cover of Suz. Since $J_{2} \leqslant S u z$, also $J_{2} \leqslant .0$.

Thus if Conway had studied the Leech lattice some five years earlier, he would have discovered a total of seven new simple groups! Unfortunately he had to settle for three. However, as consolation, his paper on .0 will stand as one of the most elegant achievements of mathematics.

As promised earlier, we conclude this section with a brief description of Thompson's construction of the group $F_{3}$. Having determined the character table of a simple group $G$ of type $F_{3}$ (a highly nontrivial task), Thompson concluded that $G$ possesses exactly one irreducible rational valued character $\chi$ of degree 248 . Since the complex Lie group $E_{8}(C)$ possesses a 248-dimensional representation, it was natural for Thompson to try to relate $G$ to the group $E_{8}(\mathbf{C})$ [205], [206].

As a further indication of this connection, if $G$ exists, then $G$ must contain a subgroup $D$ which is a nonsplit extension of $E_{32}$ by $L_{5}(2)$. Dempwolff had studied groups $D$ with such a structure and shown that at most one such 
group $D$ exists (up to isomorphism) and, in addition, every faithful representation of $D$ has degree at least 248 [49]. Furthermore, one can show that the subgroup $D$ of the presumed group $G$ must also contain a subgroup $D_{0}$, which is a nonsplit extension of $Z_{4} \times Z_{4} \times Z_{4} \times Z_{4}$ by $\hat{A}_{8}$. Moreover, if $V$ is a vector space over $C$ which affords the character $\chi$ (of degree 248) and if one sets $V_{0}=C_{V}\left(O_{2}\left(D_{0}\right)\right)$, Thompson determined that $V_{0}$ is 8-dimensional and in its action on $V_{0}, D_{0}$ preserves a certain set of 240 vectors, these vectors generating a lattice in $V_{0}$ isometric to that of a root lattice associated with the Lie algebra $E_{8}$. As a consequence, $D_{0}$ can be considered to be a subgroup of $E_{8}(\mathbf{C})$.

With such a striking conclusion, Thompson was convinced that $E_{8}(\mathbf{C})$ must contain a subgroup of the form $D$. With $P$. Smith's assistance on the computer, he was able to produce an extra element of $E_{8}(\mathbf{C})$, which together with $D_{0}$ generated $D$. Thus $E_{8}(\mathbf{C})$ does indeed contain a subgroup of the form $D$; in particular, this proved the existence of the Dempwolff group. An independent construction of $D$, without the use of computers, has been given by Griess [234]. Moreover, this showed that there existed a module $V$ of dimension 248, affording the character $\left.\chi\right|_{D}$ and that $D$ preserved a certain lattice $\Lambda$ of $V$ (whence $D \leqslant \operatorname{Aut}(\Lambda)$ ). Thompson went on to prove that $\operatorname{Aut}(\Lambda) \cong Z_{2} \times G$, thus establishing the existence of a unique simple group of type $F_{3}$.

The quadratic form $K$ on $V$ preserved by the action of $D$, not surprisingly, turned out to be the Killing form associated with the complex Lie algebra $E_{8}$. Thompson showed that $\operatorname{Aut}(\Lambda)$ preserves $K$. Using $K$ to give $V$ the structure of a Lie algebra of type $E_{8}$, Thompson also proved that $D$ was the largest subgroup of $F_{3}$ preserving this Lie multiplication. Finally as $\Lambda$ is a lattice, one can "reduce $\bmod p$ "; and Thompson showed in the case $p=3$ that $F_{3}$ preserves the corresponding Lie multiplication over $G F(3)$. Thus, in fact, $F_{3} \leqslant E_{8}(3)$.

Summarizing Thompson's remarkable results, we have

THEOREM 15. There exists a unique simple group of type $F_{3}$ and it is isomorphic to a subgroup of $E_{8}(3)$.

9. Concluding remark. There you have the 26 beautiful, but enigmatic sporadic groups with Janko's fourth group and the Fischer monster still waiting to be born. Arising out of so many unrelated contexts, is it yet possible that there is a single, coherent explanation for their existence? If so, it will require some new vision, seemingly beyond the capabilities of the present generation, to discover it. 
The proof of any classification theorem can be organized so that the last line reads:

Therefore $G$ is isomorphic to ...,

the dots to be filled in with some list of known (simple) groups. This means that we must have some effective way of recognizing-i.e., identifying-the simple $K$-groups before we can hope to prove any classification theorem. In other words, we must be able to give some set of conditions on an abstract group $G$ which are sufficient to imply that $G$ is isomorphic to a specified (simple) $K$-group $G^{*}$. Moreover, these must be intrinsic conditions, in the sense that they are attainable from an analysis of the internal structure of the group $G$. For example, the descriptions of Conway's groups in terms of the Leech lattice are extrinsic. To obtain intrinsic characterizations, one must either show that it is possible to reconstruct the lattice solely from information about their subgroups or else prove that their multiplication tables are uniquely determined by their subgroup structure.

The discussion of the known simple groups in the previous chapter has clearly indicated the three primary methods of identifying the simple groups:

A. By a presentation by generators and relations.

B. By the action of the group on a suitable geometry.

C. By a primitive permutation representation.

These methods are not really distinct, for one can usually pass from one to the other with a slight addition or variation in the argument. However, in general, the form of the recognition theorem to be established depends upon the nature of the internal conditions which one expects to impose on the group $G$. Thus the Fischer transposition hypothesis leads most naturally to recognition theorems by geometries, especially those related to rank 3 permutation groups. However, even there, in dealing with the exceptional groups over $G F(2)$, Timmesfeld found it preferable to work with generators and relations. On the other hand, for general classification theorems -especially from the point of view of centralizers of involutions-the most useful form of recognition theorem is by generators and relations, particularly for identifying the groups of Lie type.

At the present time, every known simple group with the exception of the Ree groups ${ }^{2} G_{2}\left(3^{n}\right)$ and the sporadic groups $J_{4}$ and $F_{1}$ possesses such a characterization. In this chapter we shall describe some recognition theorem for each of the known simple groups, including the partially completed characterization of the Ree groups (but excluding $J_{4}$ and $F_{1}$ ).

1. The groups of Lie type. It was Tits who first realized that the conclusions of Theorem 2.1.3 could be used as a basis for characterizing the groups of Lie type [210]. He introduced the following terminology.

Definition 1. A group $G$ is said to be a $(B, N)$-pair provided 
(1) $B, N<G$ and $G=B N B$;

(2) $B \cap N=H \triangleleft N$;

(3) $W=N / H$ is generated by a set of involution $w_{i}, 1 \leqslant i \leqslant m$;

(4) If $v_{i}$ is a representative of $w_{i}$ in $N$, then for each $v \in N$ and every $i$, $1 \leqslant i \leqslant m$, we have

$$
B v B v_{i} B \leqslant(B v B) \cup\left(B v v_{i} B\right)
$$

and

(5) $B^{v_{i}} \neq B, 1 \leqslant i \leqslant m$.

As noted earlier, these conditions imply that $W$ is a Coxeter group with $w_{i}$, $1 \leqslant i \leqslant m$, as defining set. $W$ is the $W e y l$ group of $G$ and $m$ is the rank of $G$.

For each subset $\mathcal{T}$ of $\mathscr{V}=\left\{v_{i}, 1 \leqslant i \leqslant m\right\}$, we set

$$
G_{\mathscr{T}}=\left\langle B, v_{i} \mid v_{i} \in \mathscr{T}\right\rangle \text {. }
$$

Thus $G_{\mathscr{T}}=G$ if $\mathcal{T}=\mathfrak{V}$ and $G=B$ if $\mathscr{T}=\varnothing$. If $\varnothing<\mathcal{T}<\mathfrak{V}$, we call $G_{\mathscr{g}}$ a (proper) parabolic subgroup of $G$.

We note that if we set $N_{\mathscr{T}}=N \cap G_{\mathscr{T}}$ and let $B_{\mathscr{T}}$ be the largest normal subgroup of $G_{\Im}$ contained in $B$, then $\bar{G}_{\mathscr{\sigma}}=G_{\Im} / B_{\Im}$ is a $(B, N)$-pair relative to $\bar{B}_{\mathscr{T}}, \bar{N}_{\mathscr{T}}$. The Weyl group of $\bar{G}_{\mathfrak{T}}$ has generating set $\left\{v_{i} \mid v_{i} \in \mathscr{T}\right\}$.

We associate an incidence geometry $\mathcal{G}$ related to the $G_{\mathscr{\sigma}}$ as follows. The objects of $\mathcal{G}$ are the cosets $G_{\mathscr{T}} g$ for $\mathcal{T} \subseteq \mathcal{V}$ and $g \in G$. Two objects $Y_{1}, Y_{2}$ of $G$ are said to be incident if $Y_{1} \cap Y_{2} \neq \varnothing$.

Clearly $G$ acts faithfully on the objects of $\mathcal{G}$ by right multiplication and this action preserves the incidence relation. We say that $G$ induces a group of automorphisms of the geometry $\mathcal{G}$. For example, if $G \cong L_{n}(q)$ for some $q$ and $n$, then $W \cong \Sigma_{n}$ and it can be shown that the corresponding geometry is derived from that of $\mathscr{P}_{n}(q)$, projective $n$-space over $G F(q)$. More precisely, this is the geometry of "flags" in $\mathscr{P}_{n}(q)$, a flag denoting a set of subspaces $V_{i}$, $1 \leqslant i \leqslant n$, of $\mathscr{P}_{n}(q)$ with $V_{i}$ of dimension $i$ and $V_{i}$ incident with $V_{i+1}$ for all $i$. The group $G$ acts as a group of automorphisms of this flag geometry. (Brauer's characterization of the groups $L_{3}(q)$ by the structure of the centralizer of an involution also involved the construction of the underlying projective 3-space $\mathscr{P}_{3}(q)$ (but not its flag geometry), which he built up from properties of the involutions of $G$. Ultimately he was able to show that $G$ acted as a group of projective transformations of $\mathcal{P}_{3}(q)$, which yielded at once the desired isomorphism $G \cong L_{3}(q)$.)

As the example of $L_{n}(q)$ indicates, we can view these $(B, N)$-pair geometries as generalizations of the geometries of projective $n$-space. It is known, of course, that projective space of dimension $n \geqslant 3$ is necessarily Desarguesian; and, moreover, if the space is finite, then the number of points on each line is of the form $p^{r}+1$ for some prime $p$ and the geometry is that of $\mathscr{P}_{n}\left(p^{r}\right)$. It is therefore not completely surprising that these $(B, N)$-pair geometries can be classified when the group $G$ has suitably high rank; and, furthermore, that such a classification in turn determines the possibilities for G.

It was Tits who first realized this interconnection and in a fundamental elegant paper gave a complete classification of these incidence geometries [213]. The underlying notions of "apartments" and "buildings" which Tits introduced to carry out the classification have had far reaching applicability 
to the whole theory of algebraic groups, well beyond its direct significance for finite group theory. However, as Tits' theorem is primarily a result about finite geometries and only indirectly about finite groups, we shall limit ourselves here to its statement, which we express in the following form.

THEOREM 2. If $G$ is a simple $(B, N)$-pair of rank at least 3 , then $G \in$ Chev( $p)$ for some prime $p$.

Nothing so definitive can be expected when $G$ has rank 1 or 2. In fact, to say that $G$ is a $(B, N)$-pair of rank 1 is essentially the same as asserting that $G$ is doubly transitive on the cosets of $B$. In the rank 2 case, Feit and G. Higman have obtained the following partial result by a difficult combinatorial analysis of the corresponding geometries [56].

THEOREM 3. If $G$ is a $(B, N)$-pair of rank 2, then the Weyl group of $G$ has order $4,6,8,12$, or 16 .

Each of these orders occurs. Indeed, the groups of Lie type of Lie rank 2 are the groups $L_{3}(q), P_{s p}(4, q), U_{4}(q), U_{5}(q), G_{2}(q),{ }^{3} D_{4}(q),{ }^{2} F_{4}\left(2^{n}\right)$ and the corresponding Weyl groups have orders $6,8,8,8,12,12$, and 16, respectively. The order 4 case is degenerate and occurs if $G=G_{1} \times G_{2}$, where $G_{1}, G_{2}$ are each $(B, N)$-pairs of rank 1 .

If $B$ is a Borel subgroup of $G \in \operatorname{Chev}(p)$, then $B=H P$, where $P \in$ $S y l_{p}(B)$ and $H$ is a Cartan subgroup. In particular, $P$ is nilpotent. Thus in the ranks 1 and 2 cases, one would certainly be content with a classification of $(B, N)$-pairs in which $B$ satisfied some splitting condition relative to $H$.

Definition 4. A group $G$ is called a split $(B, N)$-pair if $G$ is a $(B, N)$-pair and $B=(B \cap N) U$, where $U$ is a normal nilpotent subgroup of $B$.

Obviously Tits' theorem includes the case of split $(B, N)$-pairs of rank at least 3. The combined work of Hering, Kantor, and Seitz [113] and Kantor, Seitz [136] gives a classification of split $(B, N)$-pairs of rank 1 . Using these results, Fong and Seitz have obtained the following fundamental classification of split $(B, N)$-pairs of arbitrary rank [65].

THeORem 5. If $G$ is a simple split $(B, N)$-pair, then $G \in C h e v(p)$ for some prime $p$.

We shall say a few words about the rank 1 case in the next section as part of our discussion of doubly transitive groups. We make a few comments here about the rank 2 case. First of all, at the outset Fong and Seitz use the results of [113], [136] for the rank 1 case to determine the possible structures of a parabolic subgroup $G$. The bulk of their analysis is then aimed at showing that two parabolics of $G$ "resemble" a pair of parabolics in some group $G^{*}$ of Lie type (and Lie rank 2). The recognition portion of their argument deals only with the problem of turning resemblance into isomorphism.

The primary method used by Fong and Seitz to prove that $G \cong G^{*}$ is to show that the given resemblance conditions force $G$ to have a unique multiplication table. Since $G^{*}$ is also a group which resembles $G^{*}, G^{*}$ also has this multiplication table and so $G$ and $G^{*}$ must be isomorphic. In view of 
the double coset multiplication formulas for $G$ as a $(B, N)$-pair, it is only necessary to establish the following facts:

(a) The structures of the groups $U$ and $N$ are uniquely determined;

(b) The actions of $H$ on $U$ and of $N$ on $H$ are uniquely determined.

Theorem 5 covers every (simple) group in $C h e v(p)$ of Lie rank 2 with the single exception of the Tits group $T={ }^{2} F_{4}(2)^{\prime}$, which is of index 2 in the $(B, N)$-pair ${ }^{2} F_{4}(2)$ [212]. In proving the simplicity of $T$, Tits derived a very pretty presentation of the group ${ }^{2} F_{4}(2)$. Parrott, in attempting to give a characterization of the group $T$, first used Tits' presentation to obtain one for $T$ by a standard procedure known as the Reidemeister-Schreier method, which is a general technique for finding a presentation of a subgroup from that of a group [157].

Let $P_{1}, P_{2}$ be a pair of parabolic subgroups of ${ }^{2} F_{4}(2)$ (containing the same Sylow 2-subgroup) and let $Q_{1}, Q_{2}$ be their intersections with $T$. Using his presentation of $T$, Parrott then proved the following result.

THEOREM 6. Let $G$ be a simple group, let $S \in S y l_{2}(G)$, and let $R_{1}, R_{2}$ be 2-local subgroups of $G$ containing $S$ such that $R_{i} \cong G_{i}, i=1,2$. Then $G \cong T$.

The given presentation enabled Parrott to show that the subgroup $G_{0}=$ $\left\langle R_{1}, R_{2}\right\rangle \cong T$. It follows easily from this that either $G_{0}$ is strongly embedded in $G$ or $G_{0}=G$. Bender's theorem (see §IV.1) now forces $G=G_{0} \cong T$.

Actually Theorem 6 paraphrases only the very end of Parrott's analysis. The 2-local $Q_{1}$ is, in fact, the centralizer of a 2-central involution of $T, O_{2}\left(Q_{1}\right)$ is a metabelian group (i.e., $O_{2}\left(Q_{1}\right)^{\prime}$ is abelian) of order $2^{9}$ and $Q_{1} / O_{2}\left(Q_{1}\right)$ is a Frobenius group of order 20. Parrott's main theorem is a characterization of $T$ in terms of the approximate structure of $Q_{1}$. Thus he showed that a simple group $G$ in which the centralizer $R_{1}$ of a 2 -central involution closely resembles $Q_{1}$ is necessarily isomorphic to $T$. The bulk of the proof involves the construction of the second maximal 2-local subgroup $R_{2}$ containing a Sylow 2-subgroup of $R_{1}$ and determination of the precise structure of $R_{1}$ and $R_{2}$.

It should be noted that Thompson uses Parrott's result in his classification of $N$-groups [199].

We conclude with a brief description of a second method of identifying groups of Lie type by means of a presentation known as the Steinberg relations. It is too technical to present in complete detail; we first illustrate with the special linear group $S L\left(n, p^{m}\right)$.

Let $H$ be the Cartan subgroup consisting of diagonal matrices of determinant 1 . We let $h_{i j}(t)$ be the diagonal matrix with $0 \neq t \in G F\left(p^{m}\right)$ in the $i i$ th position and $t^{-1}$ in the $j j$ th position, and 1's elsewhere. Also let $U_{i j}$ be the elementary abelian subgroup of $S L\left(n, p^{m}\right)$ of order $p^{m}$ consisting of the matrices with l's along the diagonal and 0 's elsewhere except in the $i j$ th position, $i \neq j, 2 \leqslant i, j \leqslant n$. Then each $U_{i j}$ is irreducible as an $H$-module, $H / C_{H}\left(U_{i j}\right)$ is cyclic of order $p^{m}-1$ (and is covered by $\left\langle h_{i j}(t) \mid t \in G F\left(p^{m}\right)\right\rangle$ ) and $H / C_{H}\left(U_{i j}\right)$ transitively permutes the elements of $U_{i j}^{\#}$. We can identify $H / C_{H}\left(U_{i j}\right)$ with the multiplicative group of $G F\left(p^{m}\right)$ and $U_{i j}$ with its additive group. We thus write $U_{i j}(t), t \in G F\left(p^{m}\right)$, for the typical element of $U_{i j}$. We also set 


$$
\left.U=\left\langle U_{i j}\right| i\langle j\rangle \text { and } V=\left\langle U_{i j} \mid i\right\rangle j\right\rangle \text {. }
$$

Then $U$ and $V$ are each $H$-invariant Sylow $p$-subgroups of $S L\left(n, p^{m}\right)$, consisting, respectively, of lower and upper triangular matrices with l's on the diagonal.

Using matrix multiplication, we directly verify the following relations:

$$
\left[U_{i j}(t), U_{i j^{\prime}}\left(t^{\prime}\right)\right]=\delta_{i, j^{\prime}} U_{i^{\prime} j}\left(-t t^{\prime}\right)+\delta_{j, i^{\prime}} U_{i j^{\prime}}\left(t t^{\prime}\right)
$$

(where $\delta_{i, j}$ is the Kronecker delta), whenever $i \neq j, i^{\prime} \neq j^{\prime}$ and $i \neq j^{\prime}$ or $i^{\prime} \neq j$ (or both); and

$$
h_{i j}(t)=U_{i j}(t) U_{j i}\left(-t^{-1}\right) U_{i j}(t) U_{i j}(-1) U_{j i}(1) U_{i j}(-1) .
$$

It can be shown that $S L\left(n, p^{m}\right)$ is generated by the elements $U_{i j}(t)$ and that the relations (4), (5) together with the relations

$$
\begin{aligned}
U_{i j}(t) U_{i j}\left(t^{\prime}\right) & =U_{i j}\left(t+t^{\prime}\right) \text { and } \\
h_{i j}(t) h_{i j}\left(t^{\prime}\right) & =h_{i j}\left(t t^{\prime}\right), \quad \text { for all } t, t^{\prime} \in G F\left(p^{m}\right),
\end{aligned}
$$

give a presentation of $S L\left(n, p^{m}\right)$.

Steinberg has shown that every (universal) Chevalley group $G^{*}$ of Lie rank at least 2 has an analogous presentation in terms of the elements of two Sylow $p$-subgroups $U, V$ of $G^{*}$ ( $p$ the characteristic of $G^{*}$ ), each invariant under a given Cartan subgroup $H$ of $G^{*}$ [186]. (Each group of Lie type is a homomorphic image by a central subgroup of its universal covering group.) Corresponding to the $U_{i j}$ above and their elements, there exist root subgroups of $U, V$. These are $H$-invariant elementary subgroups of order $p^{m}$ and are labeled $x_{\alpha}(t)$, where $\alpha$ is an element (a root) of the so-called root system $\Sigma$ associated with the complex Lie algebra $\varrho$ from which $G^{*}$ has been constructed. These roots are certain linear complex representations (i.e., 1-dimensional representations) of a Cartan subalgebra of $\mathcal{L}$. In the $S L\left(n, p^{m}\right)$ case, considered above, $e$ is the algebra of complex matrices of trace 0 , the diagonal matrices in $\mathcal{L}$ are a Cartan subalgebra, and the root corresponding to $U_{i j}$ sends the matrix with diagonal complex entries $h_{1}, h_{2}, \ldots, h_{n}$ into the complex number $h_{i}-h_{j}$.

These roots can be divided into positive and negative types and $x_{\alpha}(t) \in U$ or $V$ according as $\alpha$ is positive or negative, respectively. Furthermore, given two roots $\alpha, \beta \in \Sigma$ with $\alpha+\beta \neq 0$ (this is the restriction $i \neq j^{\prime}$ or $i^{\prime} \neq j$ in (4)), the commutator $\left[x_{\alpha}(t), x_{\beta}(u)\right], t, u \in G F\left(p^{m}\right)$, has the following expression, known as the Chevalley commutator formula, which generalizes the identities of (4):

$$
\left[x_{\alpha}(t), x_{\beta}(u)\right]=\prod x_{i \alpha+j \beta}\left(c_{i j} t^{i} u^{j}\right) .
$$

Here $i, j$ are restricted to positive integral values for which $i \alpha+j \beta$ is a root and the $c_{i j}$ are integers which depend on $\alpha, \beta$, but not on $t$ and $u$. Steinberg showed that these relations together with the relations obtained by substituting an appropriate root $\alpha$ for the subscript $i j$ and $-\alpha$ for $j i$ in (5) and (6) for each $\alpha \in \Sigma$ give a presentation of $G^{*}$ (except in the case $G^{*} \cong S L\left(2, p^{m}\right)$, in which case slightly different relations are needed to obtain a presentation [186]). 
In the Chevalley groups, the root groups $x_{\alpha}(t), x_{-\alpha}(t)$ together always generate a group isomorphic to $S L\left(2, p^{m}\right)$. Thus $G^{*}$ is generated in a certain natural way from its $S L_{2}$ subgroups. Curtis has, in fact, obtained a precise set of conditions on these $S L_{2}$ subgroups which suffice to imply the Steinberg relations for the Chevalley groups [47]. Furthermore, his results give analogous conclusions for the twisted groups of Lie type. Also Phan has determined a similar set of conditions for the unitary groups, using its $\mathrm{SU}_{3}$ subgroups in place of $S L_{2}$ 's [159]. In practice, the Curtis-Phan relations make it easier to obtain a presentation for the given group $G^{*}$.

2. Doubly transitive groups. As already noted, the groups of Lie type of Lie rank 1 are doubly transitive groups, so this class of permutation groups plays an especially important role in the study of simple groups. As in the rank 2 Fong-Seitz theorem, the arguments again split into two parts: resemblance and isomorphism. Although only the latter portion is strictly concerned with recognition, we prefer to treat the entire subject at this time so as to present a coherent picture.

The Frobenius conjecture concerning fixed-point-free automorphisms of prime order arose initially from the study of transitive permutation groups $G$ on a set $\Omega$ in which only the identity fixes more than one point. Using character-theoretic arguments, Frobenius proved that the stabilizer $H$ of a point has a normal complement $U$ whose nonidentity elements are precisely those permutations in $G$ fixing no points of $\Omega$ [84, Theorem 4.5.1]. In particular, it follows that $U$ is transitive on $\Omega$. Hence, according to the definition of the term, $U$ is a regular (normal) subgroup of $G$. These conditions also imply that under conjugation, the elements of $H^{\#}$ induce fixed-point-free automorphisms of $U$. Thus $G=H U$ is a Frobenius group with kernel $U$ and complement $H$, as this notion was defined in $\S I .1$. We see then that Frobenius' conjecture is related to the nilpotency of this regular normal subgroup.

As an immediate consequence of Thompson's proof of the Frobenius conjecture, one obtains the following result about doubly transitive groups.

THEOREM 1 . If $G$ is a doubly transitive permutation group on a set $\Omega$ and only the identity element of $G$ fixes three points, then $G$ is a split $(B, N)$-pair of rank 1 with $B$ the stabilizer of a point $\alpha \in \Omega$ and $B$ is a Frobenius group. Moreover, the Frobenius complement $U$ of $B$ acts regularly on $\Omega-\{a\}$.

Indeed, if $B$ is a subgroup fixing the point $a \in \Omega$, the hypothesis implies that $B$ is transitive on $\Omega-\{a\}$ and only the identity of $B$ fixes more than one point of $\Omega-\{a\}$. Hence by the Frobenius-Thompson theorem, $B$ is a Frobenius group with nilpotent kernel $U$ and complement $H$ (where $H$ is the 1-point stabilizer of $B$ on $\Omega-\{a\}$ and hence the 2-point stabilizer of $G$ on $\Omega)$. Clearly then $U$ acts regularly on $\Omega-\{a\}$.

In the mid 1930s, Zassenhaus proved the following characterization of certain doubly transitive groups satisfying the conditions of Theorem 1 [222].

THEOREM 2. Let $G$ be a simple doubly transitive permutation group in which only the identity fixes three points and let $B$ be the stabilizer of a point If the 
Frobenius kernel of $B$ is abelian, then $G \cong L_{2}(q)$ for some q. In particular, this is the case if a 2-point stabilizer has even order.

The final assertion of the theorem is a consequence of the elementary assertion that a group admitting a fixed-point-free automorphism of period 2 is necessarily abelian. Zassenhaus' argument is essentially geometric; however, in [84, Theorem 13.3.5], I have presented a generator-relation type proof of the theorem.

Zassenhaus' theorem suggests the following terminology.

DEFINITION 3. A doubly transitive permutation group in which only the identity fixes three points is called a Zassenhaus group or, for a brevity, a Z-group.

The combined work of Feit, Ito, Suzuki, and Zassenhaus [52], [127], [189], [222] has produced a complete classification of simple $Z$-groups. It was in the course of determining those of odd degree that Suzuki was led to the discovery of the family of simple groups $S z\left(2^{n}\right)$ which bears his name. Moreover, it was out of Feit's character-theoretic analysis of $Z$-groups with 2-point stabilizers of odd order that his ideas about coherent sets of characters developed, which shortly thereafter were to play so fundamental a role in the solution of the odd order problem. Here is the main result.

THEOREM 4. If $G$ is a simple $Z$-group, then $G \cong L_{2}\left(p^{n}\right)$, $p$ a prime, $n>1$, or $S z\left(2^{n}\right), n$ odd, $n \geqslant 1$.

My book includes a proof of Feit's theorem as well as an outline of Suzuki's work on Z-groups [84, Theorem 4.6.5] and [84, §16.4]. I shall limit myself here to some general comments which will help the succeeding discussion.

Let $G$ be a split $(B, N)$-pair of rank 1 , so that $B=H U$, where $H=B \cap N$ and $U$ is a nilpotent normal subgroup of $B$. Kantor and Seitz have established the following result in [136].

THEOREM 5. B contains a normal subgroup $U_{0}$ with $U_{0} \leqslant U$ such that $B=H U_{0}$ and $H \cap U_{0}=1$.

Thus the theorem reduces the study of split $(B, N)$-pairs to the special case in which the nilpotent normal subgroup of $B$ is disjoint from $B \cap N$. For simplicity, we shall say that such a $(B, N)$-pair is strongly split. Note that by the above discussion, every $Z$-group is a strongly split $(B, N)$-pair.

Let $G$ be a simple strongly split $(B, N)$-pair, so that $B=H U$ with $H=B$ $\cap N$ and $U$ nilpotent and normal in $B$ with $H \cap U=1$. If $v$ is a representative of the generating involution of $W=N / H$, then every element $g \in G$ - $B$ has a unique representation of the form

$$
g=x u_{1} v u_{2} \text {, where } x \in H \text { and } u_{1}, u_{2} \in U .
$$

We call (1) the canonical representation of $g$.

As in the rank 2 case, the multiplication table of $G$ is completely determined by the following data (cf. (2) of the previous section. We note, however, that Fong and Seitz show that the analogue of (c) in the rank 2 case is a consequence of (a) and (b)). 
(a) The structures of $N$ and $U$;

(b) The actions of $H$ on $U$ and $v$ on $H$; and

(c) The canonical representation of the element $v u v$ for each $u \in U^{\#}$.

For $u \in U^{\#}$, we can write

$$
v u v=h(u) f(u) v g(u),
$$

where $h(u) \in H$, and $f(u), g(u) \in U$. A solution of (c) amounts to a determination of the functions $f, g, h$. Using the associative law for $G$, one obtains several, unfortunately implicit, relations among $f, g, h$. To obtain a solution of (c), we must, in effect, solve these implicit equations and find explicit expressions for $f, g$, and $h$.

Once the structure of $H$ and $U$ are determined together with the action of $H$ on $U$ and $v$ on $H$, it will clearly suffice to determine the values of $f, g$, and $h$ on some representative in each orbit of $H$ on $U^{\#}$.

For example, in the doubly transitive representation of $L_{2}(q)$ on the cosets of a Borel subgroup $B=H U$, if $v$ denotes an involution inverting $H$, there is an element $u_{0} \in U^{\#}$ such that

$$
v u_{0} v=u_{0} v u_{0} .
$$

Similarly for the analogous representation of $S z\left(2^{n}\right)$ (again $v$ inverts $H$ ), there is an element $u_{0} \in U$ of order 4 such that

$$
v u_{0} v=u_{0}^{2} v u_{0}^{2} \text {. }
$$

What we are saying is that $L_{2}(q)$ and $S z\left(2^{n}\right)$ are characterized by the structure of $B$, the action of $v$ on $H$ and equations (4) and (5), respectively -i.e., any doubly transitive group satisfying the corresponding set of conditions has a uniquely determined multiplication table and so is isomorphic to $L_{2}(q)$ or $S z\left(2^{n}\right)$, respectively. (Zassenhaus' theorem can be proved in this maner.)

In the case of simple $Z$-groups, it is not difficult to show, in general, that $H$ must be cyclic and that $H$ is inverted by $v$. Moreover, if

$$
|H| \geqslant \frac{1}{2}(|U|-1) \text {, }
$$

it follows easily that $U$ is abelian, so $G \cong L_{2}(q)$ by Theorem 2 . In the contrary case, Feit's theorem implies:

$$
U \text { is a } p \text {-group for some prime } p \text {; and }\left|U: U^{\prime}\right| \leqslant 4(|H|)^{2}+1 \text {. }
$$

Ito proved, using character theory, that there are no $Z$-groups satisfying (7) with $p$ odd. (Recent general results of Sibley on coherent sets of characters yield an easier proof of Ito's theorem.)

Suzuki, considering the case $p=2$, showed first that $H$ transitively permutes the involutions of $U$. Then, applying a theorem of G. Higman, which classifies all 2-groups admitting a fixed-point-free automorphism transitive on its involutions [116], Suzuki was able to conclude that $|U|=2^{2 n}$ for some odd $n>1$, that $|H|=2^{n}-1$, and, furthermore, that the structure of $B=H U$ is "almost" completely specified. More precisely, for each automorphism $\theta$ of $G F\left(2^{n}\right)$, there is a uniquely determined group $U(\theta)$ and a uniquely 
determined action of $H$ on $U(\theta)$, thus determining a unique extension $B(\theta)=H U(\theta)$. We say that $B$ is determined up to an element of the Galois group of $G F\left(2^{n}\right)$ (a cyclic group of order $n$ ). (The groups $B(\theta)$ are not all distinct; e.g., $B(\theta)=B\left(\theta^{-1}\right)$.)

In the groups $S z\left(2^{n}\right)$, themselves, one has

$$
\theta^{2}=2 \text {, }
$$

where 2 denotes the Frobenius automorphism $x \mapsto x^{2}$ for $x \in G F\left(2^{n}\right)$.

Using the implicit functional relations for $f, g, h$, Suzuki was ultimately able to prove that if $G$ is any $Z$-group satisfying (7) with $p=2$, then necessarily $\theta^{2}=2$; and, in addition, he obtained explicit formulas for $f, g$, and $h$, thus showing that the multiplication table of $G$ is uniquely determined (for each odd $n>1$ ). All that remained was for Suzuki actually to exhibit groups satisfying the given conditions! This he did by choosing suitable $4 \times 4$ matrices over $G F\left(2^{n}\right)$, one for each element of his canonical set of generators (see [84, p. 470]).

The groups $U_{3}(q)$ and ${ }^{2} G_{2}\left(3^{n}\right), n$ odd, are strongly split $(B, N)$-pairs of rank 1 , but are not $Z$-groups (the 3-point stabilizers having the respective orders $q+1$ and 2). In the unitary case, $U$ is of class 2 and order $q^{3}$ with $H$ cyclic of order $\left(q^{2}-1\right) / d$, where $d=$ g.c.d. $(3, q+1)$; while in the ${ }^{2} G_{2}\left(3^{n}\right)$ case, $U$ is of class 3 and order $3^{3 n}$ with $H$ cyclic of order $3^{n}-1$.

The combined efforts of O'Nan and Suzuki yield the following characterization of the unitary groups [151], [152], [190], [191].

THEOREM 5. Let $G$ be a strongly split simple $(B, N)$-pair of rank 1 satisfying the following conditions:

(a) $|G: B|=q^{3}+1$, where $q$ is a prime power;

(b) $H=B \cap N$ is cyclic of order $q^{2}-1$ or $\left(q^{2}-1\right) / 3$ with 3 dividing $q+1$.

Then $G \cong U_{3}(q)$.

The method of proof is similar in spirit to Suzuki's in the Z-group case: one must completely determine all the data listed in (2). Again $B$ is determined up to an element $\theta$ of the Galois group of $G F(q)$. However, this time one must prove

$$
\theta=1 \text {. }
$$

Although each part of the analysis is very difficult, particularly the explicit determination of $f$ and $g$, the greatest complications arise when $q$ is odd and $|H|=\left(q^{2}-1\right) / 3$. This case was brilliantly treated by O'Nan in his doctoral thesis. Included in his proof is the following preliminary result:

THEOREM 6. If $\mathcal{Q}_{q}$ denotes the natural block design associated with the group $U_{3}(q)$, then the automorphism group of $\mathcal{U}_{q}$ is isomorphic to $\operatorname{Aut}\left(U_{3}(q)\right)$.

To describe $\mathcal{Q}_{q}$, let $V$ be a three-dimension vector space over $G F\left(q^{2}\right)$ and let $\phi$ be a nondegenerate Hermitian form on $V$ (Hermitian with respect to the Frobenius automorphism $x \mapsto x^{q}, x \in G F\left(q^{2}\right)$ ). Let $I$ denote the family of isotropic one-dimensional subspaces of $V$ with respect to $\phi$. Then $I$ has $q^{3}+1$ points and the three-dimensional unitary groups $U_{3}(q), P G U(3, q)$, 
and $\operatorname{Aut}\left(U_{3}(q)\right)$ act naturally on $I$ as doubly transitive permutation groups. A block of $I$ is by definition the set of isotropic one-dimensional subspaces contained in a fixed nonisotropic two-dimensional subspace of $V$. Thus a block consists of $q+1$ elements of $I$. Moreover, there are exactly $q^{4}-q^{3}+$ $q^{2}$ blocks, every point of $I$ is contained in exactly $q^{2}$ blocks, and any two distinct points of $I$ are contained in a unique block. Together the set of points and blocks of $I$ form the unitary block design $\mathcal{Q}_{q}$ associated with $U_{3}(q)$.

A corresponding characterization of the Ree groups ${ }^{2} G_{2}\left(3^{n}\right)$ has yet to be obtained, despite the heroic efforts of Thompson to solve the problem. Thompson's analysis follows the same pattern as in the linear, Suzuki, and unitary cases. Unfortunately, even in the groups ${ }^{2} G_{2}\left(3^{n}\right)$ themselves the explicit forms of the functions $f$ and $g$ are horrendously complicated, involving literally hundreds of terms (in total contrast with the other cases). In fact, Ree manages to construct these groups using the values of $f$ and $g$ on only very special $H$-orbits of $U$ [161]. Hence a characterization of this family of groups appears to involve inherent difficulties.

Thompson's partial results are as follows [200]:

THEOREM 7. Let $G$ be a strongly split simple $(B, N)$-pair of rank 1 satisfying the following conditions:

(a) $|G: B|=3^{3 n}+1, n$ odd

(b) $H=B \cap N$ is cyclic of order $3^{n}-1$;

(c) $O(H)$ has order $\frac{1}{2}\left(3^{n}-1\right)$ and $O(H) U$ is a Frobenius group.

Then we have

(i) $n>1$ and $B$ is determined up to an element $\theta$ of the Galois group of $G F\left(3^{3 n}\right) ;$ and

(ii) If $\theta^{2}=3$ (the Frobenius automorphism of $G F\left(3^{3 n}\right)$ ), then $G \cong{ }^{2} G_{2}\left(3^{n}\right)$.

Implicit in Thompson's work is the additional fact that for each choice of $n$ and $\theta$, there exists at most one group $G$ (up to isomorphism). Using Thompson's prior analysis, Mark Hopkins, in his recent $\mathrm{Ph}$. D. thesis under Suzuki, has established this result for all $n$ and $\theta$. Furthermore, using the computer at the University of Illinois, he has also shown that, in fact, $\theta^{2}=3$ when $n \leqslant 29$. To complete the characterization of the Ree groups, it remains to force this same conclusion for arbitrary values of $n$. In his attempt to establish this equality in general, Thompson has determined a single polynomial which $\theta$ must satisfy whose degree (but not its coefficients) is independent of the value of $n$. However, it is an equation with a great many terms. Perhaps the next generation of computers will be able to show that $\theta^{2}=3$ is its unique solution. Let me emphasize that to work on this problem requires only a rudimentary knowledge of finite group theory, for it quickly reduces to specific combinatorial questions about functional equations with coefficients in $G F\left(3^{n}\right)$. Hopefully this discussion will tempt some "nonspecialist" to consider the problem.

In the meantime we must allow for this indeterminacy. Thus we shall say that a simple group satisfying the conditions of Thompson's theorem is of Ree type (of characteristic 3 ). We shall write ${ }^{2} G_{2}\left(3^{n}\right)^{*}, n$ odd, $n>1$, for any group of Ree type. Thus, for a given $n,{ }^{2} G_{2}\left(3^{n}\right)^{*}$ may conceivably include other 
groups than the Ree group ${ }^{2} G_{2}\left(3^{n}\right)$ itself. To accommodate these possible groups, we extend the definitions of $C h e v(3)$ and $K$-group to include all the groups ${ }^{2} G_{2}\left(3^{n}\right)^{*}$.

It is important to put the Ree group problem in its proper perspective. Obviously a "complete" classification of the finite simple groups requires a full determination of all groups of Ree type. Thompson's efforts clearly indicate the significance of such a determination for the structure of simple groups. As long as this question is unsettled, the classification will remain "incomplete". On the other hand, it is equally valid to assert that a solution of the Ree group problem is "essentially" irrelevant to the classification. Without a solution of the problem, the final list of groups would simply include the groups ${ }^{2} G_{2}\left(3^{n}\right) *$ rather than the groups ${ }^{2} G_{2}\left(3^{n}\right)$ and the precise relationship between these two families would remain unresolved. The crucial point is that, whatever the answer to the Ree group problem, it will have no further effect on the final list of simple groups. This is based on the fact that any property of $K$-groups needed for local analysis which can be established with the original definition of the term $\operatorname{Chev}(p)$ continues to hold under its expanded meaning. In other words, the internal structures of the groups ${ }^{2} G_{2}\left(3^{n}\right)$ and ${ }^{2} G_{2}\left(3^{n}\right)^{*}$ are "essentially" indistinguishable. From this point of view, the Ree group question is an "isolated" problem. The same is true for Fischer's monster and Janko's fourth group. The presently unresolved question of the existence of either group will have no additional effect on the classification since all analyses proceed on the assumption that these groups do exist.

Thus as a first approximation, the finite group theorists will settle for a classification theorem whose list of simple groups includes certain ambiguities of existence or nonexistence. The essence of the classification theorem is that the resulting list should include every possible simple group. To the extent that it leaves certain residual ambiguities, these can then be investigated as totally independent problems. Hopefully one will eventually obtain the desired "complete" classification, but it is entirely possible that one or more of these problems will remain unresolved indefinitely.

Bender, Hering, Kantor, O'Nan, Seitz, Shult, and Suzuki have each studied some further aspect of split $(B, N)$-pairs [18], [113], [136], [151], [152], [168], [189], [190]. Their combined efforts give a complete classification (up to the indeterminancy of the Ree groups). We state their result only in the simple case.

THEOREM 8. If $G$ is a simple split $(B, N)$-pair of rank 1 , then $G \cong$ $L_{2}(q), U_{3}(q), S z\left(2^{n}\right)$, or ${ }^{2} G_{2}\left(3^{n}\right)^{*}, n$ odd, $n>1$.

We conclude this discussion with a statement of O'Nan's fundamental structure theorem for arbitrary doubly transitive groups [154]. Here if $X$ is a simple group and $X \leqslant Y \leqslant \operatorname{Aut}(X)$, we call $Y$ a holomorph of $X$. Moreover, if $X$ is any permutation group on a set $\Omega$, we say that $X$ acts semiregularly on $\Omega$ if each orbit of $X$ on $\Omega$ has cardinality $|X|$. If, in addition, $X$ has only one orbit (whence $X$ is transitive on $\Omega$ ), then $X$ acts regularly on $\Omega$.

THEOREM 9. If $G$ is a doubly transitive permutation group acting on a set $\Omega$, 
then one of the following holds:

(i) A one-point stabilizer of $G$ on $\Omega$ is a local subgroup of $G$; or

(ii) A one-point stabilizer of $G$ on $\Omega$ is a holomorph of a simple group.

Furthermore, theorems of Holt and O'Nan determine the possibilities for $G$ in many of the local cases [124], [153].

THEOREM 10. Let $G$ be a doubly transitive permutation group on a set $\Omega$ in which no abelian normal subgroup acts regularly on $\Omega$. Suppose a one-point stabilizer $G_{1}$ of $G$ on $\Omega$ is a local subgroup of $G$ and assume one of the following conditions holds:

(a) $|\Omega|\left(=\left|G: G_{1}\right|\right)$ is odd;

(b) $G_{1}$ is solvable; or

(c) $G_{1}$ has an abelian normal subgroup which does not act semiregularly on $\Omega$.

Then $G \cong L_{n}(q)$ for some $n$ and $q, U_{3}(q)$ for some $q, S z\left(2^{m}\right)$, or ${ }^{2} G_{2}\left(3^{m}\right)^{*}$ for some odd $m$.

Assuming the local and regular normal subgroup cases can be completed, Theorem 10 gives strong indication that the determination of all simple groups would yield as a consequence a complete classification of all doubly transitive permutation groups.

3. The alternating groups. There is a classical presentation of the alternating groups, which can be used to identify them in any given classification problem. It is built up in a natural way from the symmetric group of two lower degree, using the involutions (12)(34), (12)(45), ., (12)(n-1 n) and adjoining the 3-cycle (123) to this set. Thus we have

THEOREM 1 . If the group $G$ is generated by elements $x_{1}, x_{2}, \ldots, x_{n-2}$ subject only to the relations: $x_{1}^{3}=1, x_{i}^{2}=1,2 \leqslant i \leqslant n-2,\left(x_{i} x_{i+1}\right)^{3}=1,1 \leqslant i \leqslant n$ -3 , and $\left(x_{i} x_{j}\right)^{2}=1,1 \leqslant i \leqslant n-4, i+1<j$, then $G \cong A_{n}$.

A proof of this theorem as well as of the corresponding presentation of $\Sigma_{n}$ can be found in Huppert [126, pp. 137-139].

4. The sporadic groups. The discussion of the last chapter shows that those sporadic groups constructed from the centralizer of an involution are satisfactorily characterized once both existence and uniqueness is established. Hence for this set of groups, only Janko's fourth group and the Fischer monster remain to be characterized. Likewise the rank 3 sporadic groups are satisfactorily characterized by their one-point stabilizers with specified action on the three orbits.

Thus, apart from $J_{4}$ and $F_{1}$, for which no characterizations yet exist, we are left with only the five Mathieu groups and the three Conway groups to consider.

A. The MATHIEU GRouPs. Remarkably the first characterization of $M_{11}$ is due to Jordan in 1872 , who proved

THEOREM 1. If $G$ is a quadruply transitive permutation group in which only the identity element fixes four letters, then $G \cong \Sigma_{4}, \Sigma_{5}, A_{6}$, or $M_{11}$.

This result has been extended by M. Hall to the case in which the subgroup 
fixing four letters has odd order (the group $A_{7}$ representing an added possibility) (see [102, Theorem 5.8.1]). The proof divides into the cases $n<7$ and $n \geqslant 8$, where $n$ is the number of letters on which $G$ acts. In the latter case, $n=11$ is forced and $G$ is shown to be generated by a specific set of permutations. This means that $G$ is uniquely determined from the given conditions (when $n \geqslant 8$ ); and as $M_{11}$ satisfies these conditions, it follows that $G \cong M_{11}$.

For the applications, the most useful characterization of the Mathieu groups is by their orders. Such characterizations have been proved for many other simple groups (particularly, sporadic groups) and is probably true for most simple groups (although not proved), as the orders of distinct simple groups rarely coincide.

THEOREM 2. If $G$ is a simple group of order $\left|M_{n}\right|, n=11,12,22,23$, or 24, then $G \cong M_{n}$.

Stanton, a student of Brauer's in the late 1940s, treated the cases $n=12$ and 24 in [181]. The cases $n=22,23$ were done by Janko [133] and the case $n=11$ by W. Wong [221] as part of more general classification theorems.

One can follow essentially the same proof in each case. From the order of $G$ and its simplicity, one first obtains its complete local structure and conjugacy classes of elements. From this one derives its character table. Now, using the Brauer trick, one produces a subgroup $G_{1}$ of index $n$. The permutation character on the cosets of $G_{1}$ has the form $l_{G}+\chi$, where $\chi$ is irreducible, which implies that $G$ is doubly transitive on the cosets of $G_{1}$. Finally, using this permutation representation and the character table, one forces certain elements of $G$ to be represented by specific permutations, which means that the multiplication table of $G$ is again uniquely determined. Thus $G$ is uniquely determined by its order; and as $M_{n}$ is simple of the same order as $G$, we conclude that $G \cong M_{n}$.

B. The Conway groups. Characterizations of the Conway groups by the centralizers of their involutions have involved two distinct types of recognition theorems:

(a) By construction of an associated graph.

(b) By construction of a lattice from internal properties of the group.

F. Smith has proved [176].

THEOREM 3. If $G$ is a group with $O(G)=Z(G)=1$ in which the centralizer $C$ of an involution is 2-constrained with $O_{2}(C)$ extra-special of order $2^{9}$ and $C / O_{2}(C) \cong S p(6,2)$, then $G \cong .2$.

By general methods of 2-local analysis, Smith determines the involution fusion pattern and the order of $G$ (using the Thompson order formula). Then by a delicate generator-relation argument, he shows that $G$ must contain a $(B, N)$-pair subgroup $H_{0}$, which from known theorems he is able to identify as $U_{6}(2)$. The group $H=N_{G}\left(H_{0}\right)$ is shown to be a unique extension of $U_{6}(2)$ by an outer automorphism of order 2 . Using only local considerations, he 
then proves

Proposition 4. $G$ is a primitive rank 3 permutation group on the cosets of $H$ with subdegrees 891 and 1408 .

Finally Smith proves the uniqueness of the resulting graph.

Patterson proceeds in a similar way to characterize .1 [158].

THEOREM 5. If $G$ is a group with $O(G)=Z(G)=1$ in which the centralizer $C$ of an involution is 2-constrained with $O_{2}(C)$ extraspecial of order $2^{9}$ and $C / O_{2}(C) \cong \Omega_{8}^{+}(2)$ (of index 2 in $\left.O_{8}^{+}(2)\right)$, then $G \cong .1$.

In this case the corresponding subgroup $H$ is isomorphic to $\widehat{S u z}$, the cover of $S u z$ by $Z_{3}$, which Patterson constructs inside of $G$ by generators and relations and identifies from its associated rank 3 graph. Likewise he determines the subdegrees of the permutation representation of $G$ on the cosets of $H$ (it is no longer of rank 3) and proves that the resulting graph is uniquely determined.

Perhaps a similar approach is possible for .3, but Fendel, in his dissertation under Feit [58], followed a more character-theoretic path, ultimately reducing the recognition problem to a theorem of Feit concerning groups having a rational-valued representation of degree 23 [54]. Fendel proved

THEOREM 6. If $G$ is a group with $O(G)=Z(G)=1$ in which the centralizer $C$ of an involution is isomorphic to that of a 2-central involution of .3 (thus $C \cong \widehat{S p}(6,2))$, then $G \cong .3$.

(Here $\widehat{S p}(6,2)$ denotes the covering group of $S p(6,2)$ by $Z_{2}$.)

After completing the local analysis, Fendel obtains the character table of $G$ and then argues that $G$ possesses an irreducible rational representation of degree 23. Now he can invoke the following theorem of Feit to complete the proof.

THEOREM 7. If $G$ is a group having a faithful irreducible rational-valued representation of degree 23 and $G$ has no subgroups of index 23 or 24 , then $G$ is isomorphic to a subgroup of $Z_{2} \times .2$ or $Z_{2} \times .3$.

Feit's paper on integral representations of groups, of which this theorem is only one of the important results, is one of the deepest papers ever written in the representation theory of finite groups, intertwining algebraic number theory, modular character theory, and group actions on integral lattices. In it, Feit establishes criteria for the action of a group $G$ on a lattice $\mathcal{E}$ to force the uniqueness of $\mathcal{L}$ up to isometry. Under the assumptions of Theorem 7 , he argues that there are exactly three possibilities for $\mathcal{E}$, each corresponding to a specific sublattice of the Leech lattice.

C. SPORADIC GROUPS BY CENTRALIZERS OF INVOLUTIONS. In \$2.4, we have listed the 11 centralizers of involutions which have given rise to sporadic simple groups; and we have just described how the Conway groups are determined from the centralizer of one of their involutions. Since $\boldsymbol{M}_{24}$ (which has the same centralizer of a 2-central involution as $\mathrm{He}$ ) occurs as one of the possible conclusions of Held's analysis, we have thus described 15 of the sporadic groups by centralizers of involutions and have discussed characteri- 
zations of 13 of them in terms of these centralizers (excluding $J_{4}$ and $F_{1}$ ).

Each of the remaining 11 sporadic groups have similar characterizations; and although such results involve more than solely recognition theorems, we shall state them here to round out the discussion. In each case, the involution in question is 2-central (with the exception of $M(22)$ ).

\begin{tabular}{|c|c|}
\hline group & centralizer of an involution \\
\hline$M_{11}$ & $G L(2,3) \cong \Sigma_{3} / Q_{3}$, trivial core \\
\hline$M_{12}$ & $\Sigma_{3} / Q_{8} * Q_{8}$, trivial core \\
\hline$M_{22}$ & $\Sigma_{4} / E_{16}, \Sigma_{4}$ acting faithfully \\
\hline$M_{23}$ & $L_{3}(2) / E_{16}, 2$-constrained \\
\hline$H S$ & $\Sigma_{5} / Q_{8} * Q_{8} * Z_{4}, 2$-constrained \\
\hline$M c$ & $\hat{A}_{8}$ \\
\hline Suz & $\operatorname{Psp}(4,3) / D_{8} * D_{8} * D_{8}, 2$-constrained \\
\hline$R u$ & $\Sigma_{5} / X$, where $X \cong E_{16} / Q_{8} \times E_{16}$, 2-constrained \\
\hline$M(22)$ & $\hat{U}_{6}(2)$ \\
\hline$M(23)$ & $\widehat{M(22)}$ \\
\hline$M(24)^{\prime}$ & $\hat{U}_{4}(3) \cdot 2 /\left(D_{8}\right)^{6}, 2$-constrained \\
\hline
\end{tabular}

Here $\hat{U}_{4}(3)$ denotes the cover of $U_{4}(3)$ by $Z_{3}$.

We now state

THEOREM 8. If $G$ is a simple group in which the centralizer of an involution is isomorphic to one of the 14 groups listed in this section (including those for $.1, .2, .3)$ or one of the 12 groups listed in \$II.4 (including $M_{24}$ ), then one of the following holds:

(i) $G$ is isomorphic to one of the known sporadic groups;

(ii) $G$ is of type $J_{4}$ or $F_{1}$; or

(iii) $G \cong L_{3}(3)$ or $L_{5}(2)$.

The last two groups arise from $\Sigma_{3} / Q_{8}$ and $L_{3}(2) / D_{8} * D_{8} * D_{8}$, respectively.

Many of the sporadic groups which are described here by involutions with 2-constrained centralizers contain other involutions with non 2-constrained centralizers. For example, $Z_{2} \times \Sigma_{5}, Z_{2} \times \operatorname{Aut}\left(A_{6}\right)$ and $Z_{2} \times Z_{2} \times S z(8)$ occur as centralizers of non 2-central involutions in $M_{12}, H S$, and $R u$, respectively. For the general analysis of groups of component type, it is important to have characterizations of these groups by such centralizers. However, it is better to leave this until the general discussion of "standard form" problems in Chapter VI. The same comments apply to the groups of Lie type of odd characteristic and the alternating groups. 
In this chapter we shall describe the principal methods and results which underlie local group-theoretic analysis. We shall not attempt the same for character theory, but shall limit our discussion of those techniques to the few specific places in the text in which they are needed.

It is often difficult to distinguish between a "technique" and a "classification theorem", for once a result of the latter type is proved, it becomes a tool for all subsequent classification theorems. We have not troubled ourselves here with this distinction; rather our aim is to lay out for the reader the most important general ideas of local and internal geometric analysis which have provided the basis for the major results that have been established within the four phases of the classification of simple groups.

1. Strong embedding. As we have already pointed out, the simplicity of $G$ must somehow be used to force $G$ to have an internal structure resembling that of a simple $K$-group $G^{*}$. The single most general tool for accomplishing this is by means of the construction of a strongly embedded subgroup of $G$, followed by invocation of Bender's complete classification of groups which possess a strongly embedded subgroup (almost always in order to obtain a contradiction).

There is a certain analogy between the nonexistence of strongly embedded subgroups in the general finite simple group and the nondegeneracy of the Killing form in the study of semisimple Lie algebras $\mathcal{L}$. The latter enables one to conclude that $\mathcal{L}$ has a trivial radical, which has profound effect on its internal structure. The appropriate analogue for finite groups $G$ of the radical of $\mathcal{L}$ is the core $O(G)$ of $G$. If $G$ is simple, $O(G)$ is, of course, trivial (since all groups of odd order are solvable). The nonexistence of a strongly embedded subgroup enables us to translate this condition into internal structural properties of $G$.

Typically, the argument goes as follows. To show that the core of the centralizer $C$ of an involution of $G$ resembles that of some known simple group (the nature of this resemblance will be made clear in Chapters $\mathbf{V}$ and VI), one assumes false and on the basis of this assumption constructs inside of $G$ a subgroup $M$ with nontrivial core which is strongly embedded in $G$. But a consequence of Bender's theorem (Corollary 8 below) implies that $O(M) \leqslant$ $O(G)$. However, $O(G)=1$ as $G$ is simple, contrary to the fact that $O(M) \neq 1$ by construction. Thus we can deduce significant properties of $O(C)$ from the nonexistence of a strongly embedded subgroup in $G$.

On the other hand, there are fundamental uses of Bender's theorem unrelated to cores, which occur at a later stage of the analysis, after one has shown that $G$ resembles internally some simple $K$-group $G^{*}$. This time one constructs inside of $G$ by purely group-theoretic means a subgroup $G_{0}$ isomorphic to $G^{*}$. To obtain the desired conclusion that $G \cong G^{*}$, one must obviously prove that $G_{0}=G$. Assuming this to be false, one argues that $N_{G}\left(G_{0}\right)$ is strongly embedded in $G$. Bender's theorem (Theorem 5 below) then 
yields that $G \cong L_{2}\left(2^{n}\right), U_{3}\left(2^{n}\right), S z\left(2^{n}\right)$ for some $n$. One now simply checks that none of these groups possesses a subgroup of the structure of $G_{0}$.

There are many equivalent definitions of a strongly embedded subgroup; we take the following:

DEFINITION 1. Let $X$ be a finite group and $H$ a proper subgroup of $X$ of even order. $H$ is said to be strongly embedded in $X$ provided $N_{X}(T) \leqslant H$ for every nontrivial 2-subgroup $T$ of $H$.

Clearly by Sylow's theorem, it suffices to impose the condition on subgroups $T$ of a fixed Sylow 2-subgroup $S$ of $H$.

In the terminology of Definition 1.5.11, it follows that $\Gamma_{S, 1}(X)<X$. Thus the assertion that a group $X$ has a strongly embedded subgroup is equivalent to $X$ having a proper 1-generated core.

As a consequence of the definition, one can easily prove

PROPOSITION 2. If $H$ is a strongly embedded subgroup of $X$, then the following conditions hold:

(i) If $S \in S y l_{2}(H)$, then $S \in S y l_{2}(X)$;

(ii) For any $x \in X-H,\left|H \cap H^{x}\right|$ is odd. In particular, $H=N_{X}(H)$; and

(iii) In the permutation representation of $X$ on the conjugates of $H$ in $X$, the 1-point stabilizer has even order and every 2-point stabilizer has odd order.

Further properties of groups with a strongly embedded subgroup $H$ are established in [84, \$9.2]. In particular, $X$ and $H$ each have only one conjugacy class of involutions and each coset of $H$ in $X-H$ contains precisely one involution. The latter condition implies that any conjugate $H_{1}$ of $H$ distinct from $H$ has the form $H_{1}=H^{t}$ for some involution $t$ of $X$. In addition, if $y \in \Phi(H)$, then $H=C_{X}(y) K$, where $K$ has odd order. Thus the embedding and structure of a strongly embedded subgroup is very restricted.

A particular case of the split $(B, N)$-pair rank 1 theorem, proved by Suzuki in his study of doubly transitive groups of odd degree [189] is the following:

THEOREM 3. Let $G$ be a simple group having a strongly embedded subgroup $H$. If the permutation representation of $G$ on the conjugates of $H$ is doubly transitive and if $H$ possesses a normal subgroup which acts regularly on the conjugates other than $H$, then $G \cong L_{2}\left(2^{n}\right), n \geqslant 2, S z\left(2^{2 n+1}\right), n \geqslant 1$, or $U_{3}\left(2^{n}\right)$, $n \geqslant 2$.

Bender took up the general strongly embedded problem at this point. His classification [19] proceeds in two steps. He first proves

THEOREM 4. If $G$ is a group with a strongly embedded subgroup $H$, then one of the following holds:

(i) $G$ is doubly transitive on the conjugates of $H$ in $G$; or

(ii) $G$ has cyclic or quaternion Sylow 2-subgroups and $O(G)$ is transitive on the conjugates of $H$ in $G$.

Note that as $H=N_{G}(H)$ by Proposition 2, the permutation-theoretic assertion that an element $x \in G$ fixes a given conjugate of $H$ is equivalent to the statement that $x$ lies in that conjugate.

The proof of the theorem is by induction on $|G|$ and involves a careful analysis of the normalizers in $G$ of $p$-subgroups of $H$ which lie in at least 
three conjugates of $H, p$ an odd prime. Ultimately the proof is reduced to the special case in which $G$ has cyclic or quaternion Sylow 2-subgroups (in which case $G=O(G) H$ and $H=C_{G}(z)$ for $z \in \mathscr{G}(H)$ by the Brauer-Suzuki theorem to be discussed in \$5).

Bender's main result (stated here only for simple groups) is the following.

THEOREM 5. If $G$ is a simple group with a strongly embedded subgroup $H$, then $G \cong L_{2}\left(2^{n}\right), n \geqslant 2, S z\left(2^{2 n+1}\right), n \geqslant 1$, or $U_{3}\left(2^{n}\right), n \geqslant 2$.

In view of Theorems 3 and 4 , Bender can assume that $G$ is doubly transitive on the conjugates of $H$ in $G$ and that a Sylow 2-subgroup $S$ of $H$ is not normal in $H$. Moreover, he can also take $G$ to be a minimal counterexample (to a more general theorem classifying arbitrary finite groups with a strongly embedded subgroup).

The following lemma enables Bender to apply induction.

LemMa 6. Let $Y \leqslant H$ and suppose that both $|Y \cap H|$ is even and $\left|Y \cap H^{8}\right|$ is even for some $g \in G-H$. Then $Y \cap H$ is strongly embedded in $Y$.

With the aid of the lemma, Bender proves the following key result.

Proposition 7. Let $Y$ be a subgroup of $H$ which lies in at least three conjugates of $H$. Then we have

(i) $\left|C_{H}(Y)\right|$ is odd; and

(ii) $C_{G}(Y)$ transitively permutes the set of conjugates of $H$ containing $Y$.

To establish the theorem, Bender must derive a contradiction from his assumptions. This is obtained by a careful analysis of a certain nontrivial subgroup $E$ of $H$ of odd order. Let $g \in G-H$ and set $D=H \cap H^{g}$, so that $|D|$ is odd. By Proposition $2, H=D S$. Since $S$ is not normal in $H$ by assumption, a normal subgroup $W$ of $H$ chosen minimal subject to $H=D W$ is necessarily distinct from $S$. This implies that $D \cap W \neq 1$, otherwise $|W|=|S|$ and then $W=S$. The pertinent subgroup $E$ is defined to be $D \cap W$.

Bender's theorem has the following fundamental corollary.

COROLLARY 8. If the group $G$ contains a strongly embedded subgroup $H$, then $O(H) \leqslant O(G)$.

The corollary can be viewed as a nonsimplicity criterion, since it asserts, under the assumption of strong embedding, that the core of a proper subgroup lies in the core of the entire group.

We come now to Aschbacher's generalization of Bender's strong embedding theorem to groups with a proper 2-generated core, which as we have pointed out earlier yields as a corollary the classification of nonconnected simple groups with a connected Sylow 2-group. We state Aschbacher's result only in the simple case [6].

THEOREM 9. If $G$ is a simple group with a proper 2-generated core, then $G \cong L_{2}(q), q \geqslant 3, S z\left(2^{2 n+1}\right), n \geqslant 1, U_{3}\left(2^{n}\right), n \geqslant 1, M_{11}$, or $J_{1}$.

Aschbacher considers a minimal counterexample $G$ (to a more general 
theorem classifying all groups with a proper 2-generated core). The following lemma is easily proved.

LEMMA 10. If $z$ is an involution of $G$ such that $C_{z}<G, m_{2}\left(C_{z}\right) \geqslant 3$, and $C_{z}$ has a proper 2-generated core, then $\bar{C}_{z}=C_{z} / O\left(C_{z}\right)$ has a normal subgroup $\bar{L} \cong L_{2}\left(2^{n}\right), \quad U_{3}\left(2^{n}\right), \quad S z\left(2^{n}\right), \hat{S z}(8), \quad S L_{2}(5), \quad$ or $S L(2,5) * S L(2,5) ; \quad$ and $m_{2}\left(C_{\bar{C}_{z}}(\bar{L})\right)=1$.

Here $\hat{S} z(8)$ denotes a covering group of $S z(8)$ by $Z_{2}$. Centralizers of involutions of the above general form were first considered in [95], where such involutions were called exceptional (actually our definition had not covered the cases in which $\bar{L} \cong S L(2,5)$ or $S L(2,5) * S L(2,5))$. In particular, we had there proved all but one case of the following result, which Aschbacher handles.

PROPOSITION 11. If $z$ is an exceptional involution of the simple group $X$ and $z$ is 2-central in $X$, then $X \cong J_{1}$.

This result depends in part upon Janko's characterization of $J_{1}$ by the centralizer of an involution of the form $Z_{2} \times L_{2}(4)$.

To establish his main theorem, Aschbacher reduces the problem to a second major theorem which gives a basic criterion for a group to possess a strongly embedded subgroup. The reduction is carried out as follows. First, he argues easily that a minimal counterexample $G$ (to the general theorem) is quasisimple with $O(G)=1$ and $|Z(G)| \leqslant 2$. If $G$ is not simple, a careful analysis of a Sylow 2-subgroup $\bar{S}$ of $\bar{G}=G / Z(G)$ shows that $\bar{S}$ is isomorphic to a Sylow 2-subgroup of $A_{9}$ and the fusion pattern of involutions in $\bar{G}$ is the same as that in $A_{9}$. A theorem of Harada and me [87] is now applicable and yields that $\bar{G} \cong A_{9}$. It follows at once that $G \cong \hat{A}_{9}$, which is one of Aschbacher's possible conclusions.

Thus the minimal counterexample $G$ is simple. If $m_{2}(G) \leqslant 2, G$ is determined from known classification theorems [2] and as $G$ has a proper 2-generated core, $G \cong L_{2}(q)$ for suitable odd $q$ or $M_{11}$, so $G$ is not a counterexample. Hence also $m_{2}(G) \geqslant 3$.

Let $S \in \operatorname{Syl}_{2}(G)$ and set $M=\Gamma_{S, 2}(G)$, so that $M<G$ by hypothesis. Let $z$ be an involution of $Z(S)$. Then $S \leqslant C_{z}$, so $m_{2}\left(C_{z}\right)=m_{2}(S) \geqslant 3$. If $C_{z} \$ M$, then $\Gamma_{S, 2}\left(C_{z}\right)<C_{z}$, so $C_{z}$ has a proper 2-generated core. Hence $z$ is exceptional by Lemma 10 and consequently $G \cong J_{1}$ by Proposition 11 . Again $G$ is not a counterexample. We thus conclude that $C_{z} \leqslant M$. Furthermore, by definition of $M$, clearly $M \cap M^{g}$ has cyclic or generalized quaternion Sylow 2-subgroups whenever $M \neq M^{g}$. Since $C_{z} \leqslant M$, this immediately yields that $z \in M^{g}$ if and only if $g \in M$.

Now let $u$ be any involution of $C_{z}$ such that $C_{u} \$ M$ and let $H$ be the subgroup of $C_{u}$ generated by all $G$-conjugates of $z$ which lie in $C_{u}$. It is easily seen that either $u$ is exceptional or $m_{2}\left(C_{u}\right)=2$. Now with the aid of Glauberman's $Z^{*}$-theorem (see §5), Aschbacher argues that $H \cap M$ is strongly embedded in $H$. Thus he can invoke the following theorem (which we again state only in the simple case) to conclude that $G$ has a strongly embedded subgroup (whence $G \cong L_{2}\left(2^{n}\right), S z\left(2^{n}\right)$, or $U_{3}\left(2^{n}\right)$ by Bender's theorem) and so is not a counterexample. 
THEOREM 12. Let $G$ be a simple group, $S$ a Sylow 2-subgroup of $G, M$ a proper subgroup of $G$ containing $S$, and $z$ an involution of $Z(S)$. Assume the following:

(a) $z^{g} \in M$ for $g \in G$ if and only if $g \in M$; and

(b) If $u$ is an involution of $C_{z}$ with $C_{u} \$ M$ and $H=\left\langle z^{G} \cap C_{u}\right\rangle$, then $H \cap M$ is strongly embedded in $H$

Under these conditions, $H$ is strongly embedded in $G$.

We shall not outline the proof of the theorem. In spirit it is similar to that of Bender's strong embedding argument. In particular, Aschbacher shows that a minimal counterexample $G$ is simple and acts doubly transitively on the set $\Omega$ of $G$-conjugates of $M$. To obtain a contradiction, Aschbacher must carry out a deep analysis of the structure of this doubly transitive group $G$ in terms of its action on $\Omega$. This analysis utilizes some key ideas from Shult's proof of his involution fusion theorem [169].

Recently Holt [125] and F. Smith [231] have independently determined all groups satisfying condition (a) alone. We state their result in permutation-theoretic language.

THEOREM 13. Let $G$ be a primitive permutation group on a set $\Omega$. If $O(G)=Z(G)=1$ and some 2-central involution of $G$ fixes exactly one point of $\Omega$, then either $G$ has a strongly embedded subgroup or $G \cong A_{n}$ or $\Sigma_{n}, n$ odd.

Finally combining Theorem 9 with Theorem I.5.12, we obtain the following result, which solves one of the major parts of the nonconnected simple group problem. For simplicity, we limit ourselves to the 2-rank at least 3 case.

THEOREM 14. If $G$ is a nonconnected simple group of 2-rank at least 3 with a connected Sylow 2-subgroup, then $G \cong L_{2}\left(2^{n}\right), n \geqslant 3, S z\left(2^{2 n+1}\right), n \geqslant 1, U_{3}\left(2^{n}\right)$, $n \geqslant 3$, or $J_{1}$.

2. Signalizer functors. As a first step in proving that the centralizer $C$ of an involution in an arbitrary simple group $G$ resembles the centralizer $C^{*}$ in some simple $K$-group $G^{*}$, one would naturally try to prove that the core $O(C)$ bears a close relationship to $O\left(C^{*}\right)$. However, as the following result shows, the structure of $O\left(C^{*}\right)$ is very restricted.

Proposition 1. If $G^{*}$ is a simple K-group, then the core of the centralizer of an involution of $G^{*}$ is cyclic.

In view of this result, we clearly need some general method for limiting the structure of the cores of centralizers of involutions in arbitrary simple groups. Signalizer functors represent the principal tool which has been developed to accomplish this purpose. I should like now to motivate this concept.

To do so, let us suppose that our simple group $G$ resembles internally a $K$-group $G^{*}$ (as far from simple as you wish). If $t^{*} \in \mathscr{G}\left(G^{*}\right)$, then clearly $C_{O\left(G^{*}\right)}\left(t^{*}\right) \leqslant O\left(C_{G^{*}}\left(t^{*}\right)\right)$. (For general $K$-groups, equality certainly need not hold.) If $t$ is an element of $G$ which corresponds in the "resemblance" to $t^{*}$, there "ought to be" some subgroup of $O\left(C_{G}(t)\right)=O\left(C_{t}\right)$ which corresponds to $C_{O\left(G^{*}\right)}\left(t^{*}\right)$. To conceptualize the situation, let us put $\theta\left(C_{t}\right)$ for this hypothetical subgroup and let us set 


$$
\theta(G)=\left\langle\theta\left(C_{t}\right) \mid t \in \mathscr{G}(G)\right\rangle .
$$

If $G$ truly resembles $G^{*}$, then as $G$ is simple, $G^{*}$ must be simple, too, and we should have $O\left(G^{*}\right)=1$. But then $\theta(G)=1$ and hence $\theta\left(C_{t}\right)=1$ for each $t \in \mathscr{G}(G)$. Hence our objective must be to show that each $\theta\left(C_{t}\right)$ is trivial. If the resemblance is not quite so strong, we might hope as a reasonable first approximation to show that $\theta(G)$ has odd order inasmuch as each $\theta\left(C_{t}\right)$ corresponds to a subgroup of $O\left(G^{*}\right)$. That such a conclusion would be very helpful in proving the triviality of each $\theta\left(C_{t}\right)$ can be seen by the following theorem, which deals with a special case.

THEOREM 2. Let $G$ be a simple group, $S$ a Sylow 2-subgroup of $G$, and assume the following:

(a) $W=\left\langle O\left(C_{x}\right) \mid x \in \mathscr{G}(S)\right\rangle$ is of odd order; and

(b) $C_{W}(x)=O\left(C_{x}\right)$ for every $x \in G(S)$.

Then $O\left(C_{t}\right)=1$ for every $t \in \mathscr{G}(G)$.

It will be instructive to prove this theorem since it involves a typical application of the Bender-Aschbacher results. First, as $G$ is simple, the Brauer-Suzuki theorem (see \$5) implies that $m_{2}(G) \geqslant 2$. Hence $G$ contains a four subgroup $U$. For any such $U$, set

$$
W_{U}=\left\langle O\left(C_{u}\right) \mid u \in U^{\#}\right\rangle .
$$

If $U \leqslant S$, then by (1) and assumption (b), we have

$$
W_{U}=\left\langle C_{W}(u) \mid u \in U^{\#}\right\rangle .
$$

Since $|W|$ is odd and $U$ is noncyclic abelian, it follows from (2) and Proposition I.4.7 that

$$
W=W_{U} .
$$

Furthermore, if $g \in G$, it is immediate from (1) that

$$
\left(W_{U}\right)^{g}=W_{U^{*}},
$$

Now set $N=N_{G}(W)$. We claim that

$$
\Gamma_{S, 2}(G) \leqslant N \text {. }
$$

Indeed, let $T \leqslant S$ with $m_{2}(T)>2$. We must show that $N_{G}(T) \leqslant N$ for any such $T$. It will suffice to prove that $y \in N$ for any $y \in N_{G}(T)$. Since $m_{2}(T) \geqslant 2, T$ contains a four subgroup $V$. Then $V^{y} \leqslant T$ and $V^{y}$ is also a four group. Hence (3) holds for both $V$ and $V^{y}$, whence

$$
W=W_{V}=W_{V} \text {. }
$$

But by (4), $W_{V^{y}}=\left(W_{V}\right)^{y}$, so by (6), we have

$$
W=W^{y} \text {. }
$$

Thus $y \in N=N_{G}(W)$, proving (5).

Suppose $W \neq 1$. Since $|W|$ is odd, $W<G$ and as $G$ is simple, it follows that $N=N_{G}(W)<G$. Hence $\Gamma_{S, 2}(G)<G$ by (5) and so $G$ has a proper 2 -generated core. Now Aschbacher's classification theorem yields that $G \cong$ $L_{2}(q), q>3, S z\left(2^{2 n+1}\right), n \geqslant 1, U_{3}\left(2^{n}\right), n \geqslant 2, M_{11}$ or $J_{1}$. However the precise centralizers of involutions in each of these groups is known and in none of 
them does the subgroup $W$ turn out to be nontrivial of odd order. Hence we reach a contradiction and so we conclude that $W=1$.

It follows now from (b) that $O\left(C_{x}\right)=1$ for every $x \in \mathscr{G}(S)$. Hence by Sylow's theorem, we obtain the desired conclusion $O\left(C_{t}\right)=1$ for every $t \in \mathscr{G}(G)$.

The theorem suggests that to prove that $\theta\left(C_{t}\right)=1$ for all $t \in \mathscr{G}(G)$, it would suffice to show for $S \in \operatorname{Syl}_{2}(G)$ that

$$
\begin{aligned}
& \theta(G ; S)=\left\langle\theta\left(C_{x}\right) \mid x \in \mathscr{G}(S)\right\rangle \text { has odd order; and } \\
& C_{\theta(G ; S)}(x)=\theta\left(C_{x}\right) \text { for each } x \in G(S) .
\end{aligned}
$$

There is a serious technical difficulty in trying to prove such a result directly-namely, if $x \notin Z(S)$, then $S \leqslant C_{x}$, so we do not even know at the outset that our hypothetical subgroup $\theta\left(C_{x}\right)$ is $S$-invariant. This difficulty can be overcome if instead of working with $S$, we limit ourselves to abelian subgroups $A$ of $S$, for then if $x \in \mathscr{G}(A)$, we shall at least have $A \leqslant C_{x}$. Clearly there will be no loss if we restrict ourselves to elementary abelian subgroups of $S$.

Thus we should like to be able to prove, for suitable elementary abelian $A \leqslant S$, that

$$
\begin{aligned}
& \theta(G ; A)=\left\langle\theta\left(C_{x}\right) \mid x \in A^{\#}\right\rangle \text { has odd order; and } \\
& C_{\theta(G ; A)}(x)=\theta\left(C_{x}\right) \text { for every } x \in A^{\#} .
\end{aligned}
$$

In the presence of connectedness (see Definition I.5.10), such a result will be entirely sufficient; and this will help to explain the fundamental importance of this condition. Indeed, we have

Proposition 3. Let $G$ be a connected group (or a group with a connected Sylow 2-subgroup) of 2-rank at least 3 and let $S \in S y l_{2}(G)$. If (9) holds for every abelian subgroup $A$ of $S$ with $m_{2}(A) \geqslant 3$, then (8) holds for $S$.

Proor. If $A$ is an elementary subgroup of $S$ with $m_{2}(A) \geqslant 3$ and $B$ a noncyclic subgroup of $A$, it is immediate from (9) that $\theta(G ; B)=\theta(G ; A)$. Using this equality together with the connectivity of $G$ (or $S$ ), it is an easy exercise to prove the following statement:

$$
\theta(G ; U)=\theta(G ; V)
$$

for any pair of four subgroups $U$ and $V$ of $S$. Since any involution of $S$ lies in some four subgroup of $S$, this implies that

$$
\theta(G ; S)=\theta(G ; U)
$$

for any four subgroup $U$ of $S$. Also connectivity implies that $U \leq A$ for some elementary $A \leqslant S$ with $m_{2}(A) \geqslant 3$, so $|\theta(G ; U)|$ is odd by (9) and hence $|\theta(G ; S)|$ is odd. Since any $x \in \mathscr{G}(S)$ lies in some such $A$, it also follows (using (9)) that $C_{\theta(G ; S)}(x)=\theta\left(C_{G}(x)\right)$, so (8) holds.

This entire discussion has been based on the assumption that $G$ resembles a $K$-group $G^{*}$, so that we have been implicitly assuming that a likely candidate for $\theta\left(C_{t}\right), t \in G(G)$, is actually available. How then do we identify a "good" candidate in an arbitrary simple group? Again we can use the case that $G$ resembles $G^{*}$ as a guide. Indeed, if $x^{*}, y^{*}$ are commuting involutions of $G^{*}$, 
the following equality holds

$$
C_{O\left(G^{*}\right)}\left(x^{*}\right) \cap C_{G^{*}}\left(y^{*}\right)=C_{O\left(G^{*}\right)}\left(y^{*}\right) \cap C_{G^{*}}\left(x^{*}\right),
$$

inasmuch as both of these groups are equal to $C_{O\left(G^{*}\right)}\left(\left\langle x^{*}, y^{*}\right\rangle\right)$.

This identity suggests that good candidates for $\theta$ ought to satisfy the following condition for every pair of commuting involutions $x, y$ of $G$ :

$$
\theta\left(C_{x}\right) \cap C_{y}=\theta\left(C_{y}\right) \cap C_{x} .
$$

These were the kind of considerations which first led me to the concept of a signalizer functor and to the original version of what is now known as the signalizer functor theorem [82], [83]. However, a major underlying influence was Thompson's prior analysis of maximal subgroups in the odd order paper. That analysis begins with the so-called Thompson transitivity theorem, which we can state in the following extended form (cf. [84, Theorem 8.5.4]).

THEOREM 4. Let $G$ be a group in which all p-local subgroups are p-constrained for some prime $p$. Let $P \in S y l_{p}(G)$ and let $A$ be an abelian normal subgroup of $P$ maximal under inclusion. Suppose $A$ has rank at least 3 . Then for any prime $q \neq p$, any two maximal $A$-invariant $q$-subgroups of $G$ are conjugate by an element of $C_{G}(A)$.

The condition $m_{p}(A) \geqslant 3$ is critical, so that the result only applies to primes $p$ for which $m_{p}(G) \geqslant 3$ (Definition I.5.16).

In the odd order context, this was the first step in establishing the following crucial result [57].

THEOREM 5. Let $G$ be a simple group of odd order with all proper subgroups solvable, let $P \in S y l_{p}(G)$ for some prime $p$, and let $A$ be an abelian normal subgroup of $P$ maximal under inclusion. If $m_{p}(A) \geqslant 3$, then the set of all $A$-invariant $p^{\prime}$-subgroups of $G$ generates a $p^{\prime}$-group.

Call this group $\theta(G ; A)$. For $a \in A^{\#}$, it follows from the assumptions of the theorem that $C_{\theta(G ; A)}(a) \leqslant O_{p^{\prime}}\left(C_{a}\right)$. Since $O_{p^{\prime}}\left(C_{a}\right)$ is itself an $A$-invariant $p^{\prime}$-group, equality must hold. Hence we obtain as a corollary:

COROLlaRY 6. Under the assumptions and notation of Theorem $5,\left\langle O_{p^{\prime}}\left(C_{a}\right)\right| a$ $\left.\in A^{\#}\right\rangle$ is a $p^{\prime}$-group.

Since $A \nabla P$ by hypothesis, $P$ permutes the set of all $A$-invariant $p^{\prime}$-subgroups of $G$ under conjugation and so normalizes their join $\theta(G ; A)$. Since every $P$-invariant $p^{\prime}$-subgroup of $G$ is certainly $A$-invariant, we obtain the following further corollary, which was the goal of this phase of Thompson's analysis.

COROLLARY 7. Under the assumptions and notation of Theorem $5, G$ possesses a unique maximal $P$-invariant $p^{\prime}$-subgroup.

Thompson referred to any $P$-invariant $p^{\prime}$-subgroup of $G$ as a $P$-signalizer; and the fact that there was a unique maximal $P$-signalizer in $G$ was crucial for his subsequent analysis of the maximal subgroups of $G$, the aim of which was to prove that the 2-generated $p$-core $\Gamma_{P, 2}(G)$ was a proper subgroup of $G$. 
(Here already we get a clue to the importance of strong embedding and proper 2-generated cores.)

Except for the fact that $p$ is odd, we can see how closely these results parallel the earlier discussion of the section. In fact, Thompson was able to carry these ideas over to the prime 2 as well in the case of $N$-groups. Thus signalizer functors represented an attempt to abstract the Thompson methods from the specific context in which all critical subgroups were assumed to be solvable, so that they could be applied to arbitrary simple groups (of suitably high rank). Not only was this true conceptually, but in addition, our proof of the signalizer functor theorem is very closely patterned on Thompson's proof of Theorem 5 .

Some time later, Goldschmidt [77], [78], utilizing ideas of Bender in his simplification of Thompson's odd order results [21], established a significant extension and improvement in the proof of the signalizer functor theorem. We shall follow Goldschmidt's terminology here. Since signalizer functors are also important in studying centralizers of elements of odd prime order, all definitions will be made for arbitrary primes.

Definition 8. Let $X$ be a group and $A$ an elementary abelian $p$-subgroup of $X$ for some prime $p$. Suppose that for each $a \in A^{\#}$, there is associated an $A$-invariant $p^{\prime}$-subgroup (i.e. of order prime to $\left.p\right) \theta\left(C_{X}(a)\right)$ of $C_{X}(a)$ such that for each $a, b \in A^{\#}$,

$$
\theta\left(C_{X}(a)\right) \cap C_{X}(b)=\theta\left(C_{X}(b)\right) \cap C_{X}(a) .
$$

Then $\theta$ is said to be an $A$-signalizer functor on $X$.

We set

$$
\theta(X ; A)=\left\langle\theta\left(C_{X}(a)\right) \mid a \in A^{\#}\right\rangle
$$

and call $\theta(X ; A)$ the closure of $\theta . \theta$ is said to be complete provided

$$
\begin{aligned}
& \theta(X ; A) \text { is a } p^{\prime} \text {-group; and } \\
& C_{\theta(X ; A)}(a)=\theta\left(C_{X}(a)\right) \text { for } a \in A^{\#} .
\end{aligned}
$$

$\theta$ is said to be solvable if $\theta\left(C_{X}(a)\right)$ is solvable for each $a \in A^{\#}$.

Note that if $p=2$, then each $\theta\left(C_{X}(a)\right)$ has odd order and so is solvable by the Feit-Thompson theorem. Hence in this case, every $A$-signalizer functor on $G$ is necessarily solvable.

The solvable signalizer functor theorem asserts:

THEOREM 9. Let $X$ be a group, $p$ a prime, $A$ an elementary abelian $p$-subgroup of $X$ of rank at least 3 , and $\theta$ a solvable $A$-signalizer functor on $G$. Then $\theta$ is complete.

Goldschmidt's theorem covers all cases except when $p$ is odd and $m_{p}(A)=$ 3 , a case which was subsequently treated by Glauberman [72]. Bender has given an alternate proof of Goldschmidt's theorem [22].

Since we have spent so much time motivating this theorem, we shall limit ourselves to the statement of a "generational" result which plays a key role in the proof of the theorem and which is also used to prove the existence of suitable signalizer functors. We refer the reader to Goldschmidt's highly readable solvable signalizer functor paper for a proof of the theorem. 
Proposition 10. Let $A$ be an elementary abelian p-group with $m_{p}(A) \geqslant 4$ which acts on the solvable $p^{\prime}$-group $X$. Suppose for each $D<A$ with $m_{p}(D)=$ 2 , there is given an $A$-invariant subgroup $X(D)$ of $C_{X}(D)$ such that

(a) $X=\left\langle X(D) \mid D<A, m_{p}(D)=2\right\rangle$; and

(b) $X(D) \cap C_{X}(E)=X(E) \cap C_{X}(D)$ for all $D, E \leqslant A$ with $m_{p}(D)=$ $m_{p}(E)=2$.

Then for any $D \leqslant A$ with $m_{p}(D)=2$, we have

$$
C_{X}(D)=X(D) \text {. }
$$

In particular, if $Y$ is any A-invariant subgroup of $X$, we have

$$
Y=\langle Y \cap X(D)| D\left\langle A, m_{p}(D)=2\right\rangle .
$$

Recently Lyons and I have obtained an extension of the theorem to nonsolvable signalizer functors, in which assumptions are made on the nonsolvable composition factors of the groups $\theta\left(C_{X}(a)\right)$ [92]. Our result has now been further extended by McBride [144] who has proved:

THEOREM 11. Let $X$ be a group, $p$ a prime, $A$ an elementary abelian $p$-subgroup of $X$ of rank at least 3 , and $\theta$ a nonsolvable signalizer functor on $X$. If $\theta\left(C_{X}(a)\right)$ is a $K$-group for each $a \in A^{\#}$, then $\theta$ is complete.

3. $k$-balanced groups. To make effective use of the signalizer functor theorem to study cores of centralizers in a group $G$, we clearly need methods for constructing good signalizer functors on $G$.

The so-called Thompson $A \times B$ lemma [84, Theorem 5.3.4], which is one of the basic elementary tools of local analysis, is integrally involved in the construction of essentially all signalizer functors. It asserts that if $A \times B$ acts on the $p$-group $P, p$ a prime, with $A$ a $p$-group and $B$ a $p^{\prime}$-group, then $B$ acts faithfully on $P$ if and only if it acts faithfully in $C_{P}(A)$.

For example, using it, one can immediately establish the following result.

Proposition 1. Let $G$ be a group of noncomponent type (equivalently, a group in which the centralizer of every involution is 2-contrained; see Definition I.5.9) and $A$ an elementary abelian 2-subgroup of $G$. If for each $a \in A^{\#}$, we set

$$
\theta\left(C_{a}\right)=O\left(C_{a}\right)
$$

then $\theta$ is an $A$-signalizer functor on $G$.

Suppose, in addition, that $G$ is simple and connected of 2-rank at least 3. Then the signalizer functor theorem immediately implies that the assumptions of Proposition 2.3 are satisfied and consequently also the assumptions of Theorem 2.2. It therefore follows that $O\left(C_{t}\right)=1$ for every $t \in \mathscr{G}(G)$. This shows the power of the signalizer functor method. We further remark that it is now an easy matter, again with the aid of the $A \times B$-lemma, to show that $F^{*}(H)$ is a 2-group for every 2-local subgroup $H$ of $G$, thus establishing Theorem I.5.19.

As a consequence of the proposition and the definition of a signalizer functor, we have

COROLlaRY 2. If $G$ is a group of noncomponent type, then for any pair of commuting involutions $a, b \in G$, we have 


$$
O\left(C_{a}\right) \cap C_{b}=O\left(C_{b}\right) \cap C_{a} .
$$

Definition 3. A group $G$ which satisfies (1) for every $a, b \in G(G)$ with $[a, b]=1$ is said to be balanced.

Thus the statement that $G$ is balanced is equivalent to the assertion that $\theta=O$ defines a signalizer functor on any elementary abelian 2-subgroup of $G$. Hence the results of the preceding section, when rephrased in terms of balanced groups, yield the following extension of Theorem I.5.19.

THEOREM 4. If $G$ is a connected, balanced group with $O(G)=1$ and $m_{2}(G)>3$, then $O\left(C_{t}\right)=1$ for every involution $t$ of $G$.

If $G$ is of noncomponent type, then $L\left(C_{x} / O\left(C_{x}\right)\right)=1$ for every $x \in G(G)$. When such layers are nontrivial, we cannot expect $G$ to be balanced in general. Indeed, this need not be the case even when $G$ is of known type. Thus the question of balance is related to properties of these components. We shall make this connection more precise.

DEFINITION 5. A simple group $K$ is said to be locally balanced provided whenever $K \leqslant H \leqslant \operatorname{Aut}(K)$ and $x \in \mathscr{G}(H)$, we have $O\left(C_{H}(x)\right)=1$.

The following result shows the breadth of this concept for $K$-groups.

Proposition 6. A simple group $K$ is locally balanced provided (a), (b), or (c) holds:

(a) $K \in$ Chev(2) with $K \cong L_{2}(4)$ or $L_{3}(4)$;

(b) $K \cong A_{n}$ with $n$ even, $n \geqslant 8$;

(c) $K$ is sporadic with $K \approx H e$.

Note that $L_{2}(4) \cong A_{5}$ and a transposition of $\Sigma_{5}$ has $Z_{2} \times \Sigma_{3}$ as its centralizer. Also the unitary automorphism of $L_{3}(4)$ has $U_{3}(2)$ as its fixed points, $U_{3}(2)$ being solvable with $O\left(U_{3}(2)\right) \cong Z_{3} \times Z_{3}$. Similarly, using the isomorphism $A_{6} \cong L_{2}(9)$, we check that the case $m=6$ must be excluded in (b). Likewise $\mathrm{He}$ possesses an outer automorphism of order 2 whose fixed points are a nonsplit extension of $\Sigma_{7}$ by $Z_{3}$.

Definition 7. $\mathscr{E}_{p}(G)=\left\{K \mid K\right.$ is a component of $L\left(C_{x} / O_{p^{\prime}}\left(C_{x}\right)\right), x \in$ $\left.g_{p}(G)\right\}$.

It is not difficult to prove (cf. [95]):

Proposition 8. If $G$ is a group in which every element of $\ell_{2}(G)$ is locally balanced, then $G$ is balanced.

It turns out that the nonlocally balanced simple $K$-groups satisfy conditions related to local balance. Moreover, these conditions hold for arbitrary primes and not only for the prime 2 . To state them, we require a preliminary definition.

DEFINITION 9. If $X$ is a group and $A$ an elementary abelian $p$-subgroup of $X, p$ a prime, we set

$$
\Delta_{X}(A)=\bigcap_{a \in A^{*}} O_{p^{\prime}}\left(C_{X}(a)\right) .
$$

Thus if $|A|=p, \Delta_{X}(A)=O_{p^{\prime}}\left(C_{X}(a)\right)$, where $\langle a\rangle=A$.

Definition 10. A simple group $K$ is said to be locally $k$-balanced for the prime $p, k$ a positive integer, provided whenever $K \leqslant H \leqslant A u t(K)$ and $A$ is 
an elementary abelian $p$-subgroup of $H$ of rank $k$, we have $\Delta_{H}(A)=1$.

Thus local balance is exactly the same as local 1-balance for the prime 2 . Note that if $K$ is locally $k$-balanced, then it is also locally $m$-balanced for all $m \geqslant k$. Hence the groups in Proposition 6 are locally $m$-balanced for all positive $m$ (for the prime 2).

Seitz has recently completed the analysis of local $k$-balance for odd primes for the groups of Lie type [167]. The corresponding results for the alternating groups are easy to verify. On the other hand, in the case of the sporadic groups, $\mathrm{O}^{\prime} \mathrm{Nan}$ has prepared a systematic list of various local properties of the sporadic groups [156], from which Lyons has been able to check local $k$-balance for odd primes. Likewise local $k$-balance for the prime 2 can be established from the already determined structure of the centralizers of involutions acting on simple $K$-groups.

THEOREM 11. If $K$ is a simple $K$-group, then either $K$ is locally 2-balanced for the prime $p$ or one of the following holds:

(i) $K \cong L_{p}(q), p \mid q-1$;

(ii) $K \cong U_{p}(q), p \mid q+1$;

(iii) $K \cong A_{n}$, where either $p$ is odd, $n=s p^{k}+r$ with $2 \leqslant r \leqslant p-1$, or $p=3$ and $n=s p^{k}+4$, or $p=2$ and $n=s p^{k}+3$; or

(iv) $p=5$ and $K \cong M(22)$.

One also has:

PRoposition 12. If $K \cong L_{p}(q), U_{p}(q)$, or $M(22)$, then $K$ is locally 3-balanced for the prime $p$.

The groups $A_{n}$ are exceptional. Indeed, if $n=s p^{k}+r$, with either $p$ odd and $2 \leqslant r \leqslant p-1$, or $p=2$ and $r=3$, and we take $H \cong \Sigma_{n}$; then $H$ contains an elementary abelian $p$-subgroup $A$ of rank $k$ which acts "semiregularly" on $s p^{k}$ letters and fixes the remaining $r$ letters (i.e., each element of $A^{\#}$ fixes none of the $s p^{k}$ letters). Then for each $a \in A^{\#}$, one checks that $O_{p^{\prime}}\left(C_{H}(a)\right)=Y$, where $Y \cong \Sigma_{r}$, the symmetric group on the $r$ letters fixed by $A$. Hence $Y=\Delta_{H}(A)$ if $p$ is odd, while $O_{3}(Y)=\Delta_{H}(A)$ if $p=2$; and so $\Delta_{H}(A) \neq 1$. Thus $A_{n}$ is not locally $k$-balanced for such a value of $n$.

Just as local balance on components implies global balance, the same is true of local $k$-balance.

Definition 13. A group $G$ is said to be $k$-balanced for the prime $p$ if whenever $A$ is an elementary abelian $p$-subgroup of $G$ of rank $k$ and $b$ an element of $G$ of order $p$ which centralizes $A$, we have

$$
\Delta_{G}(A) \cap C_{b} \leqslant O_{p^{\prime}}\left(C_{b}\right) .
$$

It follows that balance is the same as 1-balance for the prime 2 . We have the following extension of Proposition 8.

Proposition 14. If $G$ is a group in which every element of $\mathcal{L}_{p}(G)$ is locally $k$-balanced, then $G$ is $k$-balanced for the prime $p$.

Just as balance leads immediately to the existence of signalizer functors, so 
also one can construct signalizer functors when $G$ is $k$-balanced for the prime $p$.

PROPOSITION 15. Let $G$ be a group which is $k$-balanced for some prime $p$ and let $A$ be an elementary abelian $p$-subgroup of $G$ with $m_{p}(A) \geqslant k+2$. Suppose that $O_{p^{\prime}}\left(C_{a}\right)$ is a $K$-group (for example, solvable) for each $a \in A^{\#}$. If for $a \in A^{\#}$, we set

$$
\theta\left(C_{a}\right)=\left\langle\Delta_{G}(D) \cap C_{a} \mid D \leqslant A, m_{p}(D)=k\right\rangle,
$$

then $\theta$ is an $A$-signalizer functor on $G$.

Because of the assumption of $k$-balance, each $\theta\left(C_{a}\right) \leqslant O_{p^{\prime}}\left(C_{a}\right)$, so $\theta\left(C_{a}\right)$ is an $A$-invariant $p^{\prime}$-subgroup of $C_{a}$. Also if $k=1$, it is immediate that each $\theta\left(C_{a}\right)=O_{p^{\prime}}\left(C_{a}\right)$, whence $G$ is balanced for $p$ and so $O_{p^{\prime}}$ is an $A$-signalizer functor. If $k \geqslant 2$ and each $O_{p^{\prime}}\left(C_{a}\right)$ is solvable, one uses Proposition 2.10 to prove that $\theta$ is an $A$-signalizer functor on $G$. McBride has shown that these arguments extend to the general case of the proposition.

The theory of $k$-balance (and $L$-balance in the next section) which John Walter and I developed [97], has had a great many applications to the study of $p^{\prime}$-cores of centralizers of elements of order $p$. On the other hand, certain variations of 2-balance, introduced by Goldschmidt have produced signalizer functors which are even more effective for many classification problems; and, in particular, when $p=2$ and $\varrho_{2}(G)$ includes alternating groups which are not locally 2-balanced [79], [80]. Goldschmidt's functors are always defined in terms of commutators of suitable subgroups of $A$ on $O_{p^{\prime}}\left(C_{a}\right)$ for $a \in A^{\#}$. However, in addition to or in place of local balance assumptions on the components of $L\left(C_{a} / O_{p^{\prime}}\left(C_{a}\right)\right)$, they require assumptions on the embedding of $A$ in $C_{a}$ for $a \in A^{\#}$.

We shall give two examples, limited to the case $p=2$. The first was used by Goldschmidt in the proof of his "product fusion" theorem (see \$8) and later by Foote [67], Solomon [179], and others. It depends on the following definition.

Definition 16. Let $A$ be an elementary subgroup of $G$ of order 16 and write $A=A_{1} \times A_{2}$, where $A_{1}, A_{2}$ are four groups. We say that $G$ is core-separated with respect to the given decomposition provided for any $a \in A^{\#}$ and any component $L$ of $L\left(C_{a} / O\left(C_{a}\right)\right)$, either $A_{1}$ or $A_{2}$ centralizes $L$.

PROPOSITION 17. Suppose $G$ is core-separated with respect to the decomposition $A=A_{1} \times A_{2}$ of the elementary subgroup $A$ of $G$ of order 16. If for $a \in A^{\#}$, we set

$$
\theta\left(C_{a}\right)=\bigcap_{i=1,2}\left[O\left(C_{a}\right), A_{i}\right]\left(O\left(C_{G}\left(A_{i}\right)\right) \cap O\left(C_{a}\right)\right),
$$

then $\theta$ is an $A$-signalizer functor on $G$.

Note that no local balance conditions are required in this case, but only the core-separation property. The proof again depends on elementary generational statements (concerning the action of $A$ on subgroups of $G$ of odd order). Some time earlier Harada and I, in our study of groups with Sylow 2-groups of the form dihedral $\times$ dihedral, had used an analogous functor, but 
our proof depended upon specific properties of the components of $L\left(C_{a} / O\left(C_{a}\right)\right)$. Goldschmidt's realization that such conditions were superfluous was the basis for his completely general product fusion theorem.

The second example was used by Aschbacher in the proof of his fundamental "classical involution" theorem [10] (see \&VI.6). It depends on the following definition.

Definition 18. A simple group $K$ is said to be strongly locally 2-balanced with respect to the four subgroup $A \leqslant A u t(K)$ provided for any subgroup $H$ of $\operatorname{Aut}(K)$ containing $K A$, we have $\Delta_{H}(A)=1$ and $\left[O\left(C_{H}(a)\right), A\right]=1$ for each $a \in A^{\#}$.

This commutator condition obviously holds if $K$ is locally balanced, but for groups of Lie type of odd characteristic or alternating groups of odd degree, it depends very much upon the embedding of $A$ in $A u t(K)$. However, if $K \cong L_{2}(q), q$ odd, $q$ not a Fermat or Mersenne prime or 9, it fails for every choice of $\boldsymbol{A}$.

Proposition 19. Let $A$ be an elementary subgroup of $G$ of order 8. For each pair of elements $a, a^{\prime} \in A^{\#}$, assume

(a) $a^{\prime}$ centralizes all but at most one component $L$ of $L\left(C_{a} / O\left(C_{a}\right)\right)$;

(b) If $L$ exists and $\langle a\rangle=C_{A}(L)$, then $L / Z(L)$ is strongly locally 2-balanced with respect to $A /\langle a\rangle$.

Under these conditions, if $B$ is a fixed four subgroup of $A$ and for $a \in A^{\#}$, we set

$$
\theta\left(C_{a}\right)=\left[O\left(C_{a}\right), B\right]\left(O\left(C_{G}(B)\right) \cap C_{a}\right),
$$

then $\theta$ is an A-signalizer functor on $G$.

Note that $A$ leaves $L$ invariant and $A /\langle a\rangle$ is, in fact, a four subgroup of $\operatorname{Aut}(L / Z(L))$ in (b). The proof of the proposition is very similar to that of Proposition 17. Ordinarily, the assumption of local 2-balance on components yields a signalizer functor only when $A$ has rank at least 4 (cf. Proposition 15). The significance of Aschbacher's result is that one obtains a functor even in the rank 3 case provided one has strong local 2-balance and the embedding assumption (a) of the proposition.

4. $L$-balance. So far the discussion has focused on $p^{\prime}$-cores of centralizers of elements of order $p$ in a group $G$. The elements of $\mathcal{E}_{p}(G)$ have entered only in relation to a reduction of the question of whether $G$ is $k$-balanced for $p$ to properties of the elements of $\mathcal{L}_{p}(G)$. However, for the further analysis of the structure of the centralizers of elements of order $p$, it is important to determine the relationship between those elements of $\mathcal{L}_{p}(G)$ which occur in $L\left(C_{a} / O_{p^{\prime}}\left(C_{a}\right)\right)$ and those which occur in $L\left(C_{b} / O_{p^{\prime}}\left(C_{b}\right)\right)$ for commuting elements $a, b$ of order $p$ in $G$. We shall describe this relationship in the present section. We need some preliminary results and definitions [96], [97].

Proposition 1. Let $X$ be a group, $p$ a prime, and set $\bar{X}=X / O_{p^{\prime}}(X)$. If $\bar{K}$ is a component of $L(\bar{X})$, then $X$ contains a unique subgroup $K$ which is minimal subject to mapping on $\bar{K} . K$ is perfect and $K$ has no proper normal subgroups of $p^{\prime}$-index. 
Definition 2. If $X, \bar{X}, \bar{K}$, and $K$ are as in the proposition, we call $K$ a $p$-component of $X$. The product of all $p$-components of $X$ is called the $p$-layer of $X$ and is denoted by $L_{p^{\prime}}(X)$. For completeness, we set $L_{p^{\prime}}(X)=1$ if $X$ has no $p$-components (equivalently, if $L(\bar{X})=1$ ).

Proposition 3. For any group $X$ and any prime $p$, we have

(i) The p-components of $X$ are characterized as the set of minimal perfect subnormal subgroups of $X$ which are not $p^{\prime}$-groups;

(ii) Each p-component of $X$ is normal in $L_{p^{\prime}}(X)$; and

(iii) Every element of $X$ induces by conjugation a permutation of the set of p-components of $X$.

The following general property of finite groups is fundamental and is one form of what has come to be called L-balance [97].

THEOREM 4. For any group $G$ and any 2-subgroup $T$ of $G$, we have

$$
L_{2^{\prime}}\left(C_{G}(T)\right)<L_{2^{\prime}}(G) \text {. }
$$

The proof of this theorem, which is a fairly straightforward induction argument, depends ultimately on an important general result of Glauberman [70], concerning the automorphism group of a simple group, related to the well-known Schreier conjecture that the outer automorphism group of a simple group is necessarily solvable.

THEOREM 5. If $G$ is a simple group and $S$ a Sylow 2-subgroup of $G$, then $C_{\text {Aut }(G)}(S)$ has a normal 2-complement and, in particular, is solvable.

As a corollary of Theorem 4, one obtains the more standard form of $L$-balance.

COROLLARY 6. If $a, b$ are commuting involutions of the group $G$, then

$$
L_{2^{\prime}}\left(L_{2^{\prime}}\left(C_{a}\right) \cap C_{b}\right)=L_{2^{\prime}}\left(L_{2^{\prime}}\left(C_{b}\right) \cap C_{a}\right) \text {. }
$$

No analogue of Glauberman's theorem has been proved for odd primes. However, such an analogue does hold for simple $K$-groups. Hence the proof of Theorem 4 can be extended to yield the following two results, which are known as $L_{p^{\prime}}$-balance. (Thus $L$-balance is the same as $L_{2^{\prime}}$-balance.)

THEOREM 7. If $X$ is a $K$-group and $P$ a $p$-subgroup of $X$ for any prime $p$, then we have

$$
L_{p^{\prime}}\left(C_{X}(P)\right) \leqslant L_{p^{\prime}}(X) .
$$

COROLlARY 8. Let $G$ be a group in which all p-local subgroups are $K$-groups for some prime $p$. If $a, b$ are commuting elements of order $p$ in $G$, then

$$
L_{p^{\prime}}\left(L_{p^{\prime}}\left(C_{a}\right) \cap C_{b}\right)=L_{p^{\prime}}\left(L_{p^{\prime}}\left(C_{b}\right) \cap C_{a}\right)
$$

One can give a slightly sharper form of this last result (and likewise of Corollary 6).

Proposition 9. Let $G$ be a group in which all $p$-local subgroups are $K$-groups for some prime $p$ and let $a, b$ be commuting elements of order $p$ in $G$. Let $J$ be $a$ p-component of $L_{p}\left(L_{p}\left(C_{a}\right) \cap C_{b}\right)$ and let $K$ be the normal closure of $J$ in 
$L_{p^{\prime}}\left(C_{b}\right)$. Then we have

(i) $K$ is either a single $\langle a\rangle$-invariant p-component of $C_{b}$ or $K$ is a product of p p-components cycled by $a$; and

(ii) $J$ is a p-component of $L_{p^{\prime}}\left(C_{\left\langle K^{\langle a\rangle}\right\rangle}(a)\right)$.

In a group $G$ which is 1-balanced for $p$, we know that $O_{p^{\prime}}\left(C_{a}\right) \cap C_{b} \leqslant$ $O_{p^{\prime}}\left(C_{b}\right)$ for any pair of commuting elements $a, b$ of order $p$ in $G$. By arguments similar to those which establish the above results, one can give some description of the embedding of $O_{p^{\prime}}\left(C_{a}\right) \cap C_{b}$ in $C_{b}$ even when $G$ is not 1-balanced for $p$. We need a further definition.

Definition 10. If $X$ is a group with $O_{p^{\prime}}(X)=1$ for some prime $p$, define $L_{p^{\prime}}^{*}(X)=L(X) O_{p^{\prime}}\left(C_{X}(P)\right)$, where $P \in \operatorname{Syl}_{p}\left(L(X) O_{p}(X)\right)$. Since all choices for $P$ are conjugate by elements of $L(X)$ (by Sylow's theorem), it is immediate that $L_{p^{*}}^{*}(X)$ is determined independently of the choice of $P$. For an arbitrary group $X$, define $L_{p^{\prime}}^{*}(X)$ to be the complete inverse image in $X$ of $L_{p^{\prime}}^{*}\left(X / O_{p^{\prime}}(X)\right)$.

THEOREM 11. If $X$ is a $K$-group and $P$ is a p-subgroup of $X$ for any prime $p$, then we have

$$
O_{p^{\prime}}\left(C_{X}(P)\right) \leqslant L_{p^{\prime}}^{*}(X) .
$$

COROLLARY 12. Let $G$ be a group in which all $p$-local subgroups are $K$-groups for some prime $p$. If $a, b$ are commuting elements of $G$ of order $p$, then

$$
O_{p^{\prime}}\left(C_{a}\right) \cap C_{b} \leqslant L_{p^{\prime}}^{*}\left(C_{b}\right) \text {. }
$$

5. $p$-fusion. The term $p$-fusion, due to Brauer, refers to the conjugacy in a group $G$ of subsets of a Sylow $p$-subgroup of $G$; specifically, two such subsets are fused in $G$ if they are conjugate by an element of $G$. This is a topic with an extensive history, having its origins in the transfer homomorphism and classical results of Burnside and Frobenius which give criteria for $G$ to have a normal $p$-complement [84, Theorems 7.4.3 and 7.4.5]. There have been many extensions of their results, which provide conditions for a group $G$ to contain a normal subgroup of index $p$. When such results are applied to a simple group $G$, their effect is to force restrictions on the structure of a Sylow $p$-subgroup of $G$.

These transfer-type results deal with the "top" of a group. There is a second, fundamental theorem of Glauberman, known as the $Z^{*}$-theorem [71], which deals with its base. The $Z^{*}$-theorem is a beautiful generalization of a theorem of Brauer and Suzuki [32] which asserts that a core-free group with quaternion Sylow 2-subgroups necessarily has a center of order 2 (and hence is not simple). Together with Bender's strong embedding theorem, Glauberman's $Z^{*}$-theorem undoubtedly constitute the two most important tools of local analysis.

The $Z^{*}$-theorem has itself undergone many important generalizations in recent years. These extensions will be described in subsequent sections. But we emphasize that just as the proof of the $Z^{*}$-theorem makes explicit use of the Brauer-Suzuki theorem, so all these extensions invoke the $Z^{*}$-theorem to cover a minimal case. We should also mention that the $Z^{*}$-theorem represents the single result of general local analysis that requires Brauer's theory of modular characters. 
In the present section, we shall describe a few of the basic fusion results, which are used in local analysis and then give an application to groups of low 2-rank. The most fundamental is undoubtedly Alperin's theorem, which asserts that in any finite group, $p$-fusion is determined $p$-locally in a very precise sense [1]. (See also [84, §7.2].) There have been a number of refinements of Alperin's theorem; we shall state Goldschmidt's [76].

Definition 1 . Let $G$ be a group, $P$ a Sylow $p$-subgroup of $G$, and $\mathscr{D}$ a set of subgroups of $P$. If $A, B$ are nonempty subsets of $P$ and $g \in G$, we say that $A$ is $\mathscr{D}$-conjugate to $B$ via $g$ if there exist subgroups $D_{1}, D_{2}, \ldots, D_{n}$ in $\mathscr{D}$ and elements $g_{1}, g_{2}, \ldots, g_{n}$ in $G$ such that

(a) $g_{i} \in N_{G}\left(D_{i}\right), 1 \leqslant i \leqslant n$;

(b) $A \leqslant D_{1}$ and $A^{g_{1} \cdots g_{i}} \leqslant D_{i+1}, 1 \leqslant i \leqslant n-1$; and

(c) $g=g_{1} g_{2} \cdots g_{n}$ and $A^{g}=B$.

Furthermore, we call $\mathcal{D}$ a conjugation family (for $P$ in $G$ ) provided whenever $A$ and $B$ are nonempty subsets of $P$ which are conjugate in $G$, then $A$ and $B$ are necessarily $\mathscr{D}$-conjugate via some $g \in G$.

THEOREM 2. Let $G$ be a group, $P$ a Sylow p-subgroup of $G$, and let $\mathscr{D}$ be the set of subgroups $D$ of $P$ with the following properties:

(a) $N_{P}(D) \in S y l_{p}\left(N_{G}(D)\right)$

(b) $N_{G}(D)$ is p-constrained;

(c) $D$ maps onto $O_{p}\left(N_{G}(D) / O_{p^{\prime}}\left(N_{G}(D)\right)\right)$; and

(d) Either $D=P$ or $N_{G}(D) / D$ has a strongly $p$-embedded subgroup.

Then $\mathcal{D}$ is a conjugation family for $P$ in $G$.

Thus, in effect, the theorem asserts that any fusion (in $G$ ) of subsets of $P$ can be "factored" into a product of conjugations each of which occurs in a $p$-local subgroup of $G$ of a very particular shape.

We turn next to the prime 2 and the $Z^{*}$-theorem. However, we must first mention an elementary result of Thompson [199], known as his "transfer lemma", which has had considerable applicability in the analysis of 2-fusion, and gives a condition for a group to have a normal subgroup of index 2 .

Proposition 3. Let $G$ be a group, $S$ a Sylow 2-subgroup of $G, T$ a maximal subgroup of $S$, and $x$ an involution of $S-T$. Then either $x$ is fused in $G$ to an involution of $T$ or else $G$ has a normal subgroup $N$ of index 2 with $x \notin N$.

To state the Brauer-Suzuki theorem, we need a definition.

Definition 4. For any group $X, Z^{*}(X)$ will denote the complete inverse image in $X$ of $Z(X / O(X))$.

Clearly $Z(X / O(X))$ is an abelian 2-group and $Z^{*}(X) \nabla X$, so by the Feit-Thompson theorem, $Z^{*}(X) \leqslant \operatorname{Sol}(X)$. The $Z^{*}$-theorem (and the BrauerSuzuki particular case) gives a sufficient condition for $Z^{*}(X)$ to contain $O(X)$ properly. Since a 2-group of rank 1 is either quaternion or cyclic and since it is an easy consequence of the Burnside transfer theorem that a group with a cyclic Sylow 2-subgroup always has a normal 2-complement, the BrauerSuzuki result can be stated in the following way:

THEOREM 5. If $G$ is a group with $m_{2}(G)=1$, then $Z^{*}(G)>O(G)$. In particular, if $|G|>2$, then $G$ is not simple. 
If $G$ is a group with $m_{2}(G)=1$ and $S \in S y l_{2}(G)$, then $S$ contains only one involution $z$. Obviously then $S-\langle z\rangle$ has no involutions and so $z$ cannot fuse in $G$ to an element of $S-\langle z\rangle$. Glauberman realized that it was this property rather than the precise structure of the Sylow 2-subgroup which underlay the character-theoretic analysis of Brauer and Suzuki. Thus the following definition is needed to state his $Z^{*}$-theorem.

Definition 6. Let $G$ be a group and $S$ a Sylow 2-subgroup of $G$. An involution $z$ of $S$ is said to be isolated in $S$ (with respect to $G$ ) if $z$ is not conjugate in $G$ to any element of $S-\langle z\rangle$.

Clearly then $z$ is not conjugate in $S$ to any element of $S-\langle z\rangle$, so an isolated involution necessarily lies in $Z(S)$.

THEOREM 7. ( $Z^{*}$-THEOREM). Let $G$ be a group and $S$ a Sylow 2-subgroup of $G$. If $z$ is an isolated involution of $S$, then $z \in Z^{*}(G)$

If $G$ is simple, we see that $G$ has no isolated involutions and obviously also no normal subgroups of index 2 .

The analysis of 2 -fusion in a group is concerned primarily with the consequences of these two conditions and because of this, it applies to the wider class of so-called "fusion simple" groups.

Definition 8. A group $G$ is said to be fusion simple provided $G$ has no normal subgroups of index 2 and $Z^{*}(G)=1$.

The analysis of 2 -fusion yields very strong conclusions when $G$ has low 2-rank. We should like to illustrate this fact as well as to show how some of the above ideas are used in practice by outlining the proof of a theorem of Alperin about simple groups of 2-rank 2 [3]. (The theorem is easily extended to fusion simple groups.)

THEOREM 9. If $G$ is a simple group of 2-rank 2 and $S$ a Sylow 2-subgroup of $G$, then $S$ is either dihedral, quasi-dihedral, wreathed, or of type $U_{3}(4)$ (i.e. isomorphic to a Sylow 2-subgroup of $\left.U_{3}(4)\right)$.

We can suppose $S$ is not dihedral or quasi-dihedral, so that $S$ contains a normal four subgroup $V$ by [84, Theorem 5.4.10]. Since $V \nabla S, V$ contains an involution $z \in Z(S)$. We fix such an element $z$.

By the $Z^{*}$-theorem, there is $g \in G$ and $t \in S-\langle z\rangle$ such that $t^{g}=z$. Now let $\mathcal{D}$ be the conjugation family of Theorem 2 . We conclude from the theorem that there exists $D \in \mathscr{D}$ and $x \in N_{G}(D)$ such that

$$
u=z^{x} \neq z
$$

Since $C_{S}(D) \leqslant D$ by definition of $\mathscr{D}$, we have $z \in D$, whence $z \in Z=$ $\Omega_{1}(Z(D))$. Hence also $u=z^{x} \in Z$ as $x$ normalizes $D$ and $Z$ char $D$. Thus $Z$ is noncyclic. Since $m_{2}(D) \leqslant m_{2}(S)=2$, we conclude that

$$
Z=\Omega_{1}(D)=\langle z, u\rangle \text {. }
$$

Now let $N=N_{G}(V), C=C_{G}(V)$, and $T=S \cap C$. We have $S \in S y l_{2}(N)$ as $V \nabla S$. Thus $T \in S y l_{2}(C)$ and $|S: T| \leqslant 2$. Also $N / C$ is isomorphic to a subgroup of $\Sigma_{3}$.

We now consider two cases according as $u \in T$ or $u \notin T$. If $u \in T$, then $\langle u, V\rangle$ is elementary and again as $m_{2}(S)=2$, it follows that $u \in V$, whence 
$V=Z$. Thus $N_{G}(D)$ normalizes $V$ by (2) and so $N_{G}(D) \leq N$. Hence $u$ is conjugate to $z$ in $N$, so certainly $N \neq S C$. This implies that $N / C \cong Z_{3}$ or $\Sigma_{3}$. Now a Frattini argument yields that $N_{N}(T)$ contains a 3-element $y$ which transitively permutes the involutions of $V$. Since $V=\Omega_{1}(T), y$ thus transitively permutes the involutions of $T$. G. Higman's theorem [116] now yields that $T$ is either homocyclic abelian or of type $U_{3}(4)$.

If $T=V$, then either $S=V$ or $S$ is dihedral of order 8, contrary to assumption; so if $T$ is homocyclic abelian, then necessarily $|T| \geqslant 16$. We conclude easily in this case that either $S$ is wreathed or $S=T$. However, as $G$ is simple, a theorem of Brauer [29] shows that the latter case cannot occur. Thus the theorem holds if $T$ is homocyclic abelian. Likewise it holds if $S=T$ is of type $U_{3}(4)$. On the other hand, if $S>T$ and $T$ is of type $U_{3}(4)$, an analysis of the automorphism group of $T$ leads to a contradiction. Thus the theorem holds if $u \in T$.

Suppose finally that $u \notin T$. Then $S=T\langle u\rangle$ and $T\langle S$, so $D=E\langle u\rangle$, where $E=D \cap T$. Also $u \notin E$. But $u \in Z \leqslant Z(D)$, and consequently $D=$ $E \times\langle u\rangle$. Since $m_{2}(D) \leqslant 2$, it follows that $m_{2}(E)=1$, whence $E$ is either cyclic or quaternion. But $z \in D \cap T=E$, so $\langle z\rangle=\Omega_{1}(E)$. If $|E| \geqslant 4$, it is immediate that $\langle z\rangle$ char $D=E \times\langle u\rangle$. Clearly then $z$ and $u$ cannot be conjugate in $N_{G}(D)$. Hence we must have $|E|=2$, whence $D=\langle z, u\rangle$ is a four group. Since $C_{S}(D) \leqslant D$, we conclude now from [84, Theorem 5.4.5] that $S$ is either dihedral or quasi-dihedral, contrary to assumption.

6. Stability and characteristic subgroups for odd primes. In Corollary 2.7, we have described a basic result of the odd order paper for primes $p$ for which $m_{p}(G) \geqslant 3$ : namely, each $p \in S y l_{p}(G)$ has a unique maximal signalizer $W$ in $G$. From the preceding discussion, it is not surprising that Thompson was then able to prove, in the case $W \neq 1$, that

$$
\Gamma_{P, 2}(G) \leqslant N_{G}(W)<G .
$$

On the other hand, when $W=1$ (or as we would now say, when the signalizer functor is trivial), there is no obvious candidate for a proper subgroup of $G$ having the property that it contains $\Gamma_{P, 2}(G)$. Thompson's first proof of the existence of such a subgroup was extremely difficult. Shortly thereafter, he discovered a brilliant, conceptual proof (it is this proof which appears in the final paper), based on so-called factorization lemmas, which have had a profound impact on the development of simple group theory and which introduced for the first time what is now called the Thompson subgroup of a $p$-group. The proof then went through a second simplification, based on a theorem of Glauberman [69], which showed that for odd primes, the Thompson subgroup had certain remarkable properties, which allowed one to dispense with factorization lemmas altogether.

However, no satisfactory analogue of Glauberman's theorem is known to hold for the prime 2; whereas factorization lemmas carry over in many cases. As a result, we have the ironic twist of fate that the ideas developed for studying odd local subgroups have come to play a fundamental role in the analysis of the 2-local structure of groups of characteristic 2-type.

We leave the discussion of factorization lemmas until $\$ 10$ and focus here 
on Glauberman's achievements for odd primes. The starting point is the concept of p-stability which John Walter and I introduced to establish an extension of Thompson's odd order uniqueness results, applicable to the study of groups with dihedral Sylow 2-subgroups [93], [94].

Definition 1. Let $p$ be an odd prime and $X$ a group with $O_{p}(X)=1$. A faithful representation $\Re$ of $X$ on a vector space $V$ over $G F(p)$ is linearly $p$-stable if no $p$-element of $\mathcal{R}(X)$ has a quadratic minimal polynomial in its action on $V$. We say that $X$ is linearly $p$-stable if each such faithful representation of $X$ is linearly $p$-stable.

The natural representation of $S L\left(2, p^{n}\right)$ is not linearly $p$-stable. We note that this definition is useful only for odd primes; for if $p=2$, every element of $\Re(X)$ of order $p$ is an involution and so necessarily has a quadratic minimal polynomial.

Walter and I, using a result of Dickson's on the generation of the group $S L\left(2, p^{n}\right)[84$, Theorem 2.8.4] proved the following result.

TheOREM 2. Let $p$ be an odd prime and $X$ a group with $O_{p}(X)=1$. If $X$ is not linearly $p$-stable, then $X$ involves $S L(2, p)$ (i.e., some quotient of a subgroup of $X$ is isomorphic to $S L(2, p))$. In particular, a Sylow 2-subgroup of $X$ is neither abelian nor dihedral.

The second assertion is a consequence of the fact that $S L(2, p)$ has quaternion Sylow 2-subgroups.

We now extend this notion to the $p$-local situation. Let $Q$ be a nontrivial normal $p$-subgroup of the group $X$ and set $\bar{X}=X / \phi(Q)$, so that $\bar{Q}=$ $Q / \phi(Q)$ is elementary abelian. Setting $\tilde{X}=\bar{X} / C_{\bar{X}}(\bar{Q})$, we can view $\tilde{X}$ as a group of linear transformations of $\bar{Q}$, regarded as a vector space over $G F(p)$. If $x$ is a $p$-element of $X$ such that $[Q, x, x]=1$, then $[\bar{Q}, \tilde{x}, \tilde{x}]=1$, which in additive notation for $\bar{Q}$ asserts that $\tilde{x}$ satisfies the equation $(X-1)^{2}=0$. Hence if $\tilde{x} \neq \tilde{1}$, then $\tilde{x}$ has a quadratic minimal polynomial on $\bar{Q}$. Thus if also $O_{p}(\tilde{X})=1$, then $\tilde{X}$ is not linearly $p$-stable. These remarks serve as motivation for the following definition.

Definition 3. Let $p$ be an odd prime and $X$ a group with $O_{p^{\prime}}(X)=1$ and $O_{p}(X) \neq 1$. We say that $X$ is $p$-stable provided for every normal $p$-subgroup $Q$ of $X$ and every $p$-element $x$ of $X$ such that $[Q, x, x]=1$, if we set $\tilde{X}=X / C_{X}(Q)$, then $\tilde{x} \in O_{p}(\tilde{X})$. More generally, if $O_{p^{\prime}}(X) \neq 1$, we say that $X$ is $p$-stable if $X / O_{p^{\prime}}(X)$ is $p$-stable.

The point of the definition is that if $X$ is not $p$-stable and $O_{p^{\prime} p}(X)>O_{p^{\prime}}(X)$, it is easily shown that some section of $X$ is not linearly $p$-stable. Hence we have

THEOREM 4. If $p$ is an odd prime and $X$ is a non p-stable group with $O_{p^{\prime} p}(X)>O_{p^{\prime}}(X)$, then $X$ involves $S L(2, p)$.

Glauberman's first major result in this area, his so-called $Z J$-theorem [69, Theorem 8.2.11], deals with $p$-contrained $p$-stable groups and involves the Thompson subgroup of a $p$-group, which, in practice, has been defined in several slightly different ways. In the present context, we use the following:

Definition 5. If $P$ is a $p$-group, $p$ any prime, let $\mathscr{Q}(P)$ be the set of abelian subgroups of $\boldsymbol{P}$ of maximal order and define 


$$
J(P)=\langle A \mid A \in \mathbb{Q}(P)\rangle .
$$

Then $J(P)$ is called the Thompson subgroup of $P$. It is clearly characteristic in $P$.

For factorization lemmas, it is better to define the Thompson subgroup to be

$$
J_{e}(P)=\left\langle A \mid A \in \mathbb{Q}_{e}(P)\right\rangle,
$$

where $\mathbb{Q}_{e}(P)$ denotes the set of elementary abelian subgroups of $P$ of maximal order (equivalently, of maximal rank).

Clearly $J(P)$ char $P$. Furthermore, if $Q$ is any subgroup of $P$ containing $J(P)$, it is immediate from the definitions that $J(P)=J(Q) . J_{e}(P)$ has the same properties.

THEOREM 6. Let $p$ be an odd prime and $X$ a p-constrained group with $O_{p^{\prime}}(X)=1$. If $X$ is p-stable, then $Z(J(P))$ is a characteristic subgroup of $X$. In particular, $Z(J(P)) \nabla X$.

This is a remarkable conclusion since, even with the restriction of $p$-stability, $X$ can be fairly wild. Glauberman's proof of this theorem involves brilliant use of commutator relations. In his subsequent investigations Glauberman has pushed this technique to the status of a fine art.

To exhibit the power of this result, we present an application, which will include the $W=1$ case of the odd order paper (see \$2).

THEOREM 7. Let $G$ be a group of characteristic $p$ type in which all p-locals are $p$-stable, $p$ an odd prime. If $P \in S y l_{p}(G)$, then we have

$$
\Gamma_{P, 1}(G) \leqslant N_{G}(Z(J(P))) \text {. }
$$

Thus $N_{G}(Z(J(P)))$ is strongly $p$-embedded in $G$. If $G$ is simple of odd order with all proper subgroups solvable, it is immediate that all $p$-locals are $p$-constrained and $p$-stable. Moreover, in the $W=1$ situation, it is not difficult to prove that every $p$-local has a trivial $p^{\prime}$-core as well, so the hypotheses of the theorem are satisfied in this case.

The proof is by contradiction. Set $M=N_{G}(Z(J(P)))$ and let $H$ be a $p$-local subgroup of $G$ such that $H \nless M$ with $Q=P \cap H$ of largest possible order. Assuming the theorem is false, $Q \neq 1$. We argue that $Q \in S y l_{p}(H)$. This is clear if $Q=P$ as $P \in S y l_{p}(G)$; so we may assume $Q<P$. Then $Q<N_{P}(Q)$ and so $N_{G}(Q) \leqslant M$ by the choice of $H$ and $Q$. In particular, if $Q \leqslant R \in$ $S y l_{p}(H)$, it follows that $N_{R}(Q) \leqslant M \cap H$. Since $P \in S y l_{p}(M)$, this implies that $\left(N_{R}(Q)\right)^{x} \leqslant P$ for some $x \in M$. If $N_{R}(Q)>Q=P \cap H$, maximality of $H$ and $Q$ then yields that $H^{x} \leqslant M$, whence $H \leqslant M$, contradiction. Thus $N_{R}(Q)=Q$ and we conclude that $Q=R \in S y l_{p}(H)$.

Now Glauberman's theorem implies that $Z(J(Q)) \nabla H$, so if we set $N=$ $N_{G}(Z(J(Q)))$, we have $H \leqslant N$. If $Q=P$, then $N=M$, so $H \leqslant M$, contrary to assumption. Hence $Q<P$ and so $N_{P}(Q)>Q$. But $N_{P}(Q)$ normalizes $Z(J(Q))$ which is characteristic in $Q$, so $N_{P}(Q) \leqslant N$. Thus $P \cap N>Q$ and so $N \leqslant M$ by our maximal choice of $H$. Again $H \leqslant M$, contradiction.

With a little further arguing, Glauberman is able to draw additional conclusions, which require the following definition. 
Definition 8. Let $G$ be a group, $P$ a Sylow $p$-subgroup of $G$ for some prime $p$, and $H$ a subgroup of $G$ containing $P$. Then $H$ controls $p$-fusion in $G$ if two subsets of $P$ conjugate in $G$ are conjugate in $H$; and $H$ controls p-transfer in $G$ if the largest abelian $p$-factor of $G$ is isomorphic to that of $H$.

By the "focal subgroup theorem" [84, Theorem 7.3.4], if $H$ controls $p$-fusion in $G$, it also controls $p$-transfer. However, the converse need not be the case since less information than complete $p$-fusion is required to determine the image of $G$ in $P / P^{\prime}$ under the transfer homomorphism.

First we give a local version of Glauberman's results.

THEOREM 9. Let $X$ be a p-constrained, $p$-stable group with $O_{p^{\prime}}(X)=1$ and let $P \in S y l_{p}(X), p$ an odd prime. Then $N_{X}(Z(J(P)))$ controls $p$-fusion in $X$ and hence also $p$-transfer. In particular, $X$ has a normal $p$-complement if and only if $N_{X}(Z(J(P)))$ does.

The final assertion follows from control of $p$-transfer together with a theorem of Tate [196] (or from control of $p$-fusion together with Frobenius' normal $p$-complement theorem).

Using Theorem 9 and induction on $|G|$, Glauberman obtains the following criterion for a group $G$ to have a normal $p$-complement (cf. [84, Theorem 8.3.1]).

TheOREM 10. Let $P$ be a Sylow p-subgroup of the group, $p$ an odd prime. If $N_{G}(Z(J(P)))$ has a normal p-complement, then so also does $G$.

The celebrated Frobenius conjecture, Thompson's proof of which began the whole show, is an immediate consequence of the theorem.

THEOREM 11. If a group $G$ admits an automorphism $\phi$ of prime order $q$ fixing only the identity element of $G$, then $G$ is nilpotent.

Let $G$ be a minimal counterexample. If $H$ is a nontrivial proper characteristic subgroup of $G$, then $\phi$ acts on both $H$ and $G / H$; and is easily shown to be fixed-point-free on $G / H$. By the minimality of $G, H$ and $G / H$ are both nilpotent, so $G$ is solvable. However, it follows by elementary representation theory arguments that the theorem holds for solvable groups. Hence no such characteristic subgroup $H$ exists.

Obviously $G$ is not a 2-group, as it is not nilpotent. Let $p$ be an odd prime divisor of $|G|$. By the previous paragraph, $O_{p^{\prime}}(G)=O_{p}(G)=1$ (as each is characteristic in $G)$. It is easily proved that $\phi$ leaves invariant some Sylow $p$-subgroup $p$ of $G$, whence $\phi$ also leaves $N=N_{G}(Z(J(P)))$ invariant. Since $O_{p}(G)=1, Z(J(P))$ is not normal in $G$, so $N<G$. Hence $N$ is nilpotent by the minimality of $G$. In particular, $N$ has a normal $p$-complement, so $G$ does as well by Theorem 10. Thus $G=O_{p^{\prime}}(G) P=P$ is nilpotent, contradiction.

If $P \in S y l_{p}(G)$, it is immediate from Alperin's fusion theorem that any subgroup of $G$ containing $\Gamma_{P, 1}(G)$ controls $p$-fusion in $G$. Hence as a corollary of Theorem 7, we have

THEOREM 12. Let $G$ be a group of characteristic $p$ type in which all p-locals are $p$-stable, $p$ an odd prime. If $P \in S y l_{p}(G)$, then $N_{G}(Z(J(P)))$ controls $p$-fusion and p-transfer in $G$. 
This entire discussion suggests several interesting general questions.

(A) If $G$ is a group of characteristic $p$ type and $H$ is a $p$-local subgroup of $G$, under what conditions is $H$ contained in a $p$-local subgroup of $G$ which also contains some Sylow $p$-subgroup of $G$ ?

(B) If $G$ is a group and $P \in S y l_{p}(G), p$ a prime, is there a "functorially defined" nonidentity characteristic subgroup $K$ of $P$ for which $N_{G}(K)$ controls $p$-fusion or at least $p$-transfer in $G$ ?

(C) What can be said about the structure of a group $X$ with $O_{p}(X)=1$, $p$ an odd prime, which is not linearly $p$-stable?

(A) is concerned with the problem of "pushing up" $p$-local subgroups, which is an essential part of any theorem which asserts that a group $G$ of characteristic $p$ type has a proper 2-generated $p$-core or strongly $p$-embedded subgroup. The primary interest occurs for $p=2$ and the known results will be discussed in $\$ 12$.

The general answer to (B) is negative for $p$-fusion-the groups $G L\left(3, p^{n}\right)$ providing an easy counterexample. $p$-stability seems to be an essential requirement. Remarkably Glauberman has shown that an affirmative answer for $p$-transfer holds under very broad circumstances [73]. In fact, the only restriction is $p \geqslant 5$. The proof of this result involves extremely subtle commutator calculations coupled with tremendous ingenuity. To state it, we require a definition which extends some of the properties of the Thompson subgroup.

Definition 13. A p-conjugacy functor $K$ on a group $G, p$ a prime, is a mapping $K: P \mapsto K(P)$ defined on the set of $p$-subgroups $P$ of $G$ with the following properties:

(a) $K(P) \leqslant P$;

(b) If $P \neq 1$, then $K(P) \neq 1$; and

(c) For any $g \in G, K\left(P^{g}\right)=(K(P))^{g}$.

It is immediate that the map $P \mapsto J(P), P$ a $p$-subgroup of $G$, defines a p-conjugacy functor on $\boldsymbol{G}$.

TheOREM 14. Let $G$ be a group, $p \geqslant 5$ a prime, and $P \in \operatorname{Syl}_{p}(G)$. Then there is a p-conjugacy functor $K$ on $G$ such that $N_{G}(K(P))$ controls p-transfer in $G$, Moreover, $K(P)$ char $P$.

Glauberman defines for any $p$-group $P$ two chains of characteristic subgroups of $P$, one increasing and the other decreasing, the terminal member of either of which can be taken as $K(P)$. We shall not attempt to describe them explicitly here nor shall we discuss the many other important results which Glauberman has established in this direction. We mention only that a corollary of the last theorem, when combined with the Burnside $p^{a} q^{b}$-theorem (for the case of order $2^{a} 3^{b}$ ), proves the following long-standing conjecture.

THEOREM 15. If $G$ is a group in which every Sylow subgroup is its own normalizer, then $G$ is not simple.

Thompson was the first to study (C) in a general setting (Theorem 2 above can be viewed as a particular case of (C)) and he obtained a complete 
solution when $p \geqslant 5$ [203]. One may as well assume that $X$ itself is a group of linear transformations of a vector space $V$ over $G F(p)$, that $X$ acts irreducibly on $V$, and that $X$ is generated by the set of $p$-elements $D$ of $X$ with quadratic minimal polynomial on $V$. Thompson calls $(X, V)$ a quadratic pair under these conditions.

Note that if $x \in D$, then the quadratic condition implies that $x^{p}=1$, so $x$ has order $p$.

THEOREM 16. If $(X, V)$ is a quadratic pair for the prime $p \geqslant 5$, then we have

(i) $X$ is semisimple with quasisimple components $X_{i}, 1 \leqslant i \leqslant m, V$ is the tensor product of subspaces $V_{i}, 1 \leqslant i \leqslant m$, and $\left(X_{i}, V_{i}\right)$ is a quadratic pair for each $i, 1 \leqslant i \leqslant m$; and

(ii) Each $X_{i} \in \operatorname{Chev}(p)$ (excluding $\left.E_{8}\left(p^{r}\right)\right), 1 \leqslant i \leqslant m$.

Near the beginning of his analysis, Thompson proves the following result, which shows that the hypothesis here is closely related to the Fischer-type situation. For simplicity, we state it only in the case that $D$ is a single conjugacy class of elements of $X$.

Proposition 17. If $x, y \in D$, then either $\langle x, y\rangle$ is a p-group or $\langle x, y\rangle \cong$ $S L\left(2, p^{n}\right)$ for some $n$.

There are now several proofs of Thompson's quadratic pair theorem, each of which ends up constructing the underlying geometry (equivalently, the $(B, N)$-pair structure) from properties of $D$. Thompson makes explicit use of the module $V$; but Stark has shown [182], using Thompson's sharpened form of Proposition 17, that the theorem can be reduced to a purely group-theoretic characterization of the groups of Lie type of odd characteristic by centralizers of involutions (Aschbacher's "classical involution" theorem [10] (see §VI.6)).

The case $p=3$ is very interesting since a number of noncharacteristic 3-groups have quadratic pair representations. Thompson's former student Ho has nearly completed the analysis in this case [119]-[123]. (Also his results include a proof of Thompson's theorem.) The essential difficulty is that the analogue of Proposition 17 includes the following groups:

$$
\text { 3-groups, } \quad S L\left(2,3^{n}\right), \quad S L(2,3) \times Z_{3} \text {, and } S L(2,5) \text {. }
$$

Thus there are a greater number of possibilities for the underlying "geometry" when $p=3$.

In Thompson's analysis, the "root" subgroups play a basic role. We define them here only when $D$ is a single conjugacy class. If $x \in D$, set

$$
D_{x}=\{y \in D \mid V(y-1)=V(x-1)\} \text { and } E_{x}=D_{x} \cup\{1\} .
$$

Then it is easily shown that $E_{x}$ is an elementary abelian $p$-group. $E_{x}$ is called a root subgroup of $X$. Since $D$ is a single conjugacy class, $\left|E_{x}\right|$ is independent of the choice of $x \in D$.

Ho has proved

THEOREM 18. If $(X, V)$ is a quadratic pair for $p=3$ and the root subgroups of $X$ have order exceeding 3, then the conclusions of Thompson's quadratic pair theorem hold with $p=3$. 
Thus it is the case of root subgroups of order 3 which leads to groups outside of $C h e v(3)$. In this case, if $E=\langle x\rangle$ and $F=\langle y\rangle$ are root subgroups of $X$, then (3) can be refined to the statement

$$
\begin{aligned}
& \langle E, F\rangle \cong Z_{3}, Z_{3} \times Z_{3} \text {, a nonabelian group of order } 27 \text { and } \\
& \text { exponent } 3, S L(2,3), S L(2,5) \text {, or } S L(2,3) \times Z_{3} .
\end{aligned}
$$

Ho has proved

THEOREM 19. Let $(X, V)$ be a quadratic pair for $p=3$ with $X$ quasisimple and assume that the root groups of $X$ have order 3 . If $\langle E, F\rangle \nRightarrow S L(2,3) \times Z_{3}$ for every pair of root groups $E, F$ of $X$, then one of the following holds:

(i) $X \in$ Chev(3) (excluding $E_{8}\left(3^{r}\right), r \geqslant 1$ );

(ii) $X \cong \hat{A}_{n}, n \geqslant 5$;

(iii) $X \cong U_{n}(2), S p(6,2), D_{4}(2)$, or $G_{2}(4)$; or

(iv) $X \cong J_{2}$, Suz, or .1.

Thus only the $S L(2,3) \times Z_{3}$ case of root groups of order 3 remains open to complete the classification of all quadratic pairs.

7. The Bender method, small class Sylow 2-groups, and strong closure. Bender has developed an alternate method for studying the cores of centralizers of involutions in simple groups, based on ideas growing out of his simplified proof of Thompson's uniqueness theorems of the odd order paper, which Bender [20] first applied to give a very short proof of John Walter's classification of simple groups $G$ with abelian Sylow 2-subgroups [214]. Walter's proof, predating signalizer functors, followed the same general approach the two of us had developed for investigating groups with dihedral Sylow 2-subgroups [93] (based upon Thompson's original odd order paper argument) and was technically extremely difficult. (Bender subsequently used his method to simplify large portions of the dihedral theorem as well [23].)

The case $m_{2}(G)=2$ is covered by the dihedral theorem combined with a result of Brauer (both discussed in the next chapter); so one can suppose $m_{2}(G) \geqslant 3$ in a minimal counterexample. The signalizer functor method (which can be used here, too, but with considerable effort in the case $m_{2}(G)=3$ ) is essentially constructive-i.e., one constructs a proper subgroup $M$ of $G$ which, if not strongly embedded, at least controls a critical amount of 2 -fusion in $G$. By contrast, Bender focuses directly on a maximal subgroup $M$ of $G$ containing $C$ for some $x \in \mathscr{G}(G)$. The question of whether $M$ controls suitable 2-fusion related to the conjugacy class of $x$ is then reduced to properties of $F^{*}(M)$ and its embedding in $G$. If $O(F(M))$ is not a $p$-group, Bender establishes powerful uniqueness theorems with respect to suitable subgroups of $O(F(M))$ of nonprime power order and the entire argument is very smooth. The argument is equally smooth if either $O_{2}\left(F^{*}(M)\right) \neq 1$ or $F^{*}(M)>F(M)$. However, the remaining case, in which $F^{*}(M)$ is a $p$-group for some odd prime $p$, requires special treatment. On the other hand, the cases $m_{2}(G)=3$ and $m_{2}(G) \geqslant 4$ are treated uniformly throughout. As expected, the analysis of the subgroup structure of $G$ uses several specific properties of $K$-groups with abelian Sylow 2-groups.

The abelian Sylow 2-group theorem asserts the following. 
THEOREM 1. If $G$ is a simple group with abelian Sylow 2-subgroups, then $G \cong L_{2}(q), q \equiv 3,5(\bmod 8), L_{2}\left(2^{n}\right), n \geqslant 2, J_{1}$, or ${ }^{2} G_{2}\left(3^{n}\right)^{*}, n$ odd,$n>1$.

Goldschmidt has obtained an important extension of Bender's proof of this result, which simultaneously generalizes Glauberman's $Z^{*}$-theorem as well as a deep theorem of Shult on the weak closure of an involution in its centralizer [169]. To state it, we need the following definition.

Definition 2. Let $G$ be a group, $P$ a Sylow $p$-subgroup of $G, p$ any prime, and $Q$ a subgroup of $P$. We say that $Q$ is strongly closed in $P$ with respect to $G$ provided whenever $x \in Q$ and $x^{g} \in P$ for $g \in G$, we have $x^{g} \in Q$.

Obviously $P$ itself is strongly closed in $P$. If $\Omega_{1}(P)$ is elementary abelian, then clearly it consists precisely of the set of all elements of $P$ of order $p$ together with the identity of $P$ and so is strongly closed in $P$ with respect to $G$. In particular, this is the case if $P$ is abelian or if $p=2$ and $P$ is quaternion.

Goldschmidt was interested in the case in which a Sylow 2-subgroup $S$ of a group $G$ contained a nontrivial strongly closed abelian subgroup $A$. If $S$ is abelian, we can take $A=S$ and if $S$ has an isolated involution $x$, we can take $A=\langle x\rangle$. Goldschmidt proved [79].

THEOREM 3. Let $G$ be a simple group and $S$ a Sylow 2-subgroup of $G$. If $S$ contains a nontrivial abelian subgroup $A$ which is strongly closed in $S$ with respect to $G$, then either $S$ is abelian or $G$ has a strongly embedded subgroup. Thus $G \cong L_{2}\left(2^{n}\right), n \geqslant 2, U_{3}\left(2^{n}\right), n \geqslant 2, S z\left(2^{n}\right), n$ odd, $n>1, L_{2}(q), q \equiv 3,5$ $(\bmod 8), J_{1}$, or ${ }^{2} G_{2}\left(3^{n}\right)^{*}, n$ odd,$n>1$.

The primary difference between the Goldschmidt and the Bender situations is that in the abelian problem all proper subgroups of $G$ are $K$-groups, whereas Goldschmidt is only able to assert this inductively for certain proper subgroups of $G$. Namely, if $H<G$ and $B=H \cap A^{g} \neq 1$ for some $g \in G$, then $\left\langle B^{H}\right\rangle$ is a $K$-group. This makes for a more delicate analysis of the 2-fusion of elements of $A$ and the structure of the corresponding maximal subgroup $M$. Aschbacher's extension of Bender's strong embedding theorem (Theorem 1.9) is invoked at a critical juncture and enables Goldschmidt to conclude that $G=\left\langle C_{a} \mid a \in A^{\#}\right\rangle$.

The Bender method is so powerful when it is applicable that there have been attempts (notably by Thompson) to use it in place of signalizer functors for studying cores of centralizers of involutions in arbitrary simple groups. Unfortunately the case in which $F^{*}(M)$ is a $p$-group for some odd prime $p$ has so far constituted an insurmountable obstacle. The difficulty arises when $M$ is not $p$-stable, in which case the Glauberman $Z J$-theorem is not applicable. This problem does not occur for Theorems 1 and 3, since in those cases it is immediate that $G$ has no proper $S L(2, p)$ sections.

There is one other general classification problem to which the Bender method can easily be applied: namely, in groups with class 2 Sylow 2-groups, which Gilman and I have carried out-however, using signalizer functors [68]. Although, a priori, $S L(2, p)$ can occur as a proper section of a minimal counterexample $G$ in this problem, we were able to eliminate this possibility very easily by showing in such a case that a Sylow 2-subgroup $S$ of $G$ had a nontrivial strongly closed abelian subgroup and then invoked Goldschmidt's 
theorem to derive a contradiction. There is no doubt that the Bender method would have provided a shorter proof when $G$ was of component type. However, we deliberately chose the signalizer functor approach in the hope of gaining further insight into the general centralizer of involution core problem.

On the other hand, both approaches leave the identical problems to face when $G$ is of characteristic 2 type. In the corresponding case of the abelian Sylow 2-group problem, it is immediate that a Sylow 2-normalizer is strongly embedded in $G$, so $G$ is determined directly from Bender's theorem. (This particular case is actually covered by an earlier result of Feit [53].) However, a considerably more involved argument is required in the class 2 case, which depends in a critical way on Thompson type factorization lemmas. Using them, one pins down the structure of the maximal 2-local subgroups of $\boldsymbol{G}$ and ultimately argues that $G$ is a $(B, N)$-pair of Lie rank 2 .

Here is the statement of the class 2 theorem.

THEOREM 4. If $G$ is a simple group with Sylow 2-subgroups of class 2, then $G \cong L_{2}(q), q \equiv 7,9(\bmod 16), A_{7}, S z\left(2^{n}\right), n$ odd $, n>1, U_{3}\left(2^{n}\right), n \geqslant 2, L_{3}\left(2^{n}\right)$, $n \geqslant 2$, or Psp $\left(4,2^{n}\right), n \geqslant 2$.

The groups $L_{2}(q)$ and $A_{7}$ arise in the dihedral case and the groups $S z\left(2^{n}\right)$, $U_{3}\left(2^{n}\right)$ when $G$ has a strongly embedded subgroup.

A fuller discussion of the signalizer functor method will be given in Chapter VI.

8. Product fusion and strong closure. In the course of determining all simple groups $G$ with a given type of Sylow 2-group, one is of ten forced to consider a subsidiary problem involving the classification of groups with Sylow 2-subgroup $S$ of the form $S=S_{1} \times S_{2}$ with $S_{i} \neq 1, i=1,2$. Harada and I were the first to face this situation in our study of groups with Sylow 2-groups isomorphic to those of $G_{2}(q), q$ odd [88]. If $t$ is a 2-central involution of such a group $G, C=C_{t}, \bar{C}=C / O(C)$ and $\tilde{C}=\bar{C} /\langle\bar{t}\rangle$, our analysis of the 2-fusion of $G$ enabled us to show that $\tilde{C}$ had a normal subgroup $\tilde{X}$ of index 2 with Sylow 2-subgroup $\tilde{S}=\tilde{S}_{1} \times \tilde{S}_{2}$, where $\tilde{S}_{1}, \tilde{S}_{2}$ are (isomorphic) dihedral groups. Thus to pin down the structure of $\tilde{C}$ and hence of $\bar{C}$, we had first to determine the possibilities for $\tilde{X}$. (The existence of such an $\tilde{X}$ was expected, for if $G=G_{2}(q)$, then $C$ has a normal subgroup $X$ of index 2 with $X \cong$ $S L(2, q) * S L(2, q)$. Thus $X /\langle t\rangle \cong L_{2}(q) \times L_{2}(q)$ and $L_{2}(q)$ has dihedral Sylow 2-subgroups for odd $q$.)

If $\tilde{S}$ is abelian, the possibilites for $\tilde{X}$ follow from the abelian Sylow 2-group classification theorem; so one can restrict to the nonabelian case. Harada and I established the following result [86].

THEOREM 1 . If $G$ is a group with no nontrivial normal subgroups of odd order or odd index and $G$ has a nonabelian Sylow 2-subgroup $S$ of the form $S=S_{1} \times S_{2}$ with $S_{1}$ and $S_{2}$ dihedral, then

(i) $G=G_{1} \times G_{2}$ for suitable subgroups $G_{1}, G_{2}$ of $G$; and

(ii) For a suitable factorization of $S, S_{i} \in S y l_{2}\left(G_{i}\right), i=1,2$.

The point of (ii) is that the factorization of $S$ is by no means unique. Since $G_{1}$ and $G_{2}$ have dihedral Sylow 2-subgroups, their possible structures are 
determined from the dihedral theorem and hence so are those of $G$. Note also that the restriction on normal subgroups of odd order or index is clearly necessary to obtain a direct product factorization of $G$.

An important first step in the proof of Theorem 1 is the following fusion assertion.

PRoposition 2. For a suitable factorization of $S, S_{i}$ is strongly closed in $S$ with respect to $G, i=1,2$.

This is a natural result, since if $G=G_{1} \times G_{2}$ with $S_{i} \in \operatorname{Syl}_{2}\left(G_{i}\right)$, then indeed $S_{i}$ is strongly closed in $S$ with respect to $G$. It also suggests the following general definition.

Definition 3. Let $S \in S y l_{2}(G)$ for any group $G$. If $S=S_{1} \times S_{2}$ with $S_{i} \neq 1$ strongly closed in $S$ with respect to $G, i=1,2$, we say that $G$ has product fusion with respect to the given decomposition of $S$.

In the context of the $G_{2}(q)$ problem as well as in other similar classification problems, not only does the analysis of 2 -fusion yield the existence of $\tilde{X}$ with Sylow 2-subgroup $\tilde{S}$ of the form $\tilde{S}=\tilde{S}_{1} \times \tilde{S}_{2}$, but also the fact that $\tilde{X}$ has product fusion with respect to an appropriate factorization of $\tilde{S}$. Hence for the applications, it would have sufficed to assume $G$ has product fusion in Theorem 1.

Once the fusion pattern of $G$ was determined, Harada and I were able to use signalizer functors and Bender's strong embedding theorem to prove the existence of the required normal subgroups $G_{1}, G_{2}$. Soon after this, F. Smith [174], [175] treated the dihedral $\times$ quasi-dihedral and quasi-dihedral $\times$ quasi-dihedral cases in similar fashion, followed by D. Mason [143] who considered dihedral $\times$ wreathed, etc. In each case, construction of the required signalizer functor used local balance properties of the elements of $\mathcal{L}_{2}(G)$ (which had either dihedral, quasi-dihedral, wreathed, or quaternion Sylow 2-groups and so were $K$-groups by prior classification theorems). Each of these product fusion theorems has had corresponding application to some classification problems.

Some time later, Harris and I, investigating groups with Sylow 2-subgroups isomorphic to those of $P s p(6, q), q$ odd, [111] were forced to treat two subsidiary product fusion problems-dihedral $\times$ dihedral $\times$ dihedral and Psp (4, q)-type $\times$ dihedral. It was obviously time to consider the general case!

We succeeded in establishing a completely general product fusion theorem, under the assumption that suitable sections of 2-local subgroups of $G$ satisfied suitable local balance conditions, known to hold for all simple $K$-groups [90]. It is unnecessary to state our result explicitly, since very shortly thereafter Goldschmidt greatly improved upon it by showing that the balance assumption was superfluous if one worked with core-separated signalizer functors (see Proposition 3.17) rather than $k$-balanced functors [80]. He was thus able to prove the following lovely result.

THEOREM 4. If $G$ is a group with no nontrivial normal subgroups of odd order or of odd index and $G$ has product fusion with respect to the decomposition $S=S_{1} \times S_{2}$ of the Sylow 2-subgroup $S$ of $G$, then $G=G_{1} \times G_{2}$ with $S_{i} \in S y l_{2}\left(G_{i}\right), i=1,2$. 
With a little additional effort, Goldschmidt established a definitive result about arbitrary strongly closed 2-groups. Here the term $X^{(\infty)}$ denotes the ultimate term of the derived series of the group $X$.

THEOREM 5. Let $G$ be a corefree group, $S$ a Sylow 2-subgroup of $G$, and $T$ a subgroup of $S$ which is strongly closed in $S$ with respect to $G$. Then $\left(C_{G}(T)\right)^{(\infty)}$ $\nabla G$.

9. Weak closure and trivial intersection sets. We should like now to describe the fundamental group-theoretic consequences which Timmesfeld has obtained from his root involution theorem. To state them, we need the notion of weak closure.

Definition 1. Let $G$ be a group, $H$ a subgroup of $G$, and $K$ a subgroup or subset of $H$. Set

$$
V\left(\operatorname{ccl}_{G}(K) ; H\right)=\left\langle K^{g} \mid K^{g} \leqslant H, g \in G\right\rangle .
$$

(ccl denotes "conjugacy class" here). We call $V\left(\operatorname{ccl}_{G}(K) ; H\right)$ the weak closure of $K$ in $H$ with respect to $G$. If $V\left(\operatorname{ccl}_{G}(K) ; H\right)=K$, we say simply that $K$ is weakly closed in $H$ (with respect to $G$ ).

Very often one is interested in the case that $H=P$ is a Sylow $p$-subgroup of $G$ for some prime $p$. In that case, if $K$ is strongly closed in $P$, it is clearly weakly closed. The Thompson subgroup $J(P)$ (likewise $J_{e}(P)$ ) is an example of a weakly closed subgroup of $P$. There is a classical theorem of Grün which asserts that if $Z(P)$ is weakly closed in $P$, then the largest abelian $p$-factor group of $G$ is isomorphic to that of $N_{G}(Z(P))$ [84, Theorem 7.5.2].

Shult studied groups generated by a class of involutions $D$ such that for $x \in D$, the weak closure of $x$ in its centralizer $C_{x}$ is an abelian 2-group [269]. Timmesfeld obtained as a consequence of his root involution theorem the following partial extension of Shult's results.

THEOREM 2. Let $G$ be a group with $O(G)=1$ and $Z(G)=1$ which is generated by a conjugacy class $D$ of involutions such that

(a) For any $x \in D$, the weak closure of $x$ in $C_{x}$ is a 2-group of class at most 2; and

(b) The product of any two distinct commuting elements of $D$ is an element of D.

Then $G \cong L_{2}(q), U_{3}(q), S z(q), L_{3}(q),{ }^{3} D_{4}(q), q=2^{n}, G_{2}(q), q=2^{n}, n>1$, $A_{6}$ or $J_{2}$.

The theorem is proved by arguing that $D$ is a class of root involutions and then invoking the root involution theorem, checking afterwards which groups on the list satisfy the given hypotheses. To prove that $D$ is indeed a class of root involutions, we must show that if $x, y \in D$ with $|x y|=2 k$ for some $k\rangle 1$, then necessarily $k=2$. Now $\langle x, y\rangle$ is a dihedral group of order $4 k$ and so $Z(\langle x, y\rangle)=\langle z\rangle$ has order 2 . One checks from the structure of $\langle x, y\rangle$ that there is a conjugate $u$ of $y$ in $\langle x, y\rangle$ such that $\langle x, u\rangle$ is a four group with $z=x u$. But then $u \in D$ and so by (b), also $z \in D$. Thus $\langle x, y\rangle \leqslant C_{z}$ and so by (a), $\langle x, y\rangle$ is a 2-group of class at most 2, which immediately forces $k=2$, as required.

Timmesfeld's next major result represents a basic extension of Theorem 2 
[209]. At the same time, it generalizes Glauberman's $Z^{*}$-theorem, which corresponds to the case $|A|=2$. We state the theorem only for simple groups.

THEOREM 3. Let $G$ be a simple group and $A$ a nontrivial elementary abelian 2-subgroup of $G$. If $A$ is weakly closed in $C_{a}$ with respect to $G$ for each $a \in A^{\#}$, then one of the following holds:

(i) $G \cong L_{n}(q), S z(q), U_{3}(q), q=2^{n}$;

(ii) $G \cong A_{n}, 6<n<9$; or

(iii) $G \cong M_{22}, M_{23}, M_{24}, \mathrm{He}$.

The first step in the proof is to pin down the possible structures of the group $\left\langle A, A^{g}\right\rangle$ for $g \in G$ when $A \cap N_{G}\left(A^{g}\right) \neq 1$. With this information, Timmesfeld is able to determine the normal subgroup $X$ of $N=N_{G}(A)$ generated by the conjugates in $N$ of $A^{8} \cap N$. In particular, he shows that the hypotheses of his theorem are satisfied in $X / A$ for the image of $A^{g} \cap X$ and so induction can be applied. In this way, he ultimately establishes the following result.

Proposition 4. Suppose $A \cap N_{G}\left(A^{g}\right) \neq 1$ for some $g \in G$ and let $X$ denote the normal closure of $A^{8} \cap N_{G}(A)$ in $N_{G}(A)$. Then either $X / A \cong L_{r}\left(2^{m}\right), A_{6}$, $A_{7}, A_{8}$ for some $r$ and $m$ or else $O(X / A) \neq 1$.

Also the order of $A$ and the action of $X / A$ are determined when $X / A$ is simple. In the linear case, with $X / A \supsetneqq L_{3}(2)$ or $L_{4}(2)$, Timmesfeld argues that the conjugacy class determined by any element of $A^{\#}$ is a class of root involutions of $G$, in which case $G \cong L_{r+1}\left(2^{m}\right)$ by the root involution theorem.

On the other hand, if $X / A \cong L_{3}(2), L_{4}(2), A_{6}, A_{7}$, or $A_{8}$, Timmesfeld proves that $X=N_{G}(A)$. Since $N_{G}(A)$ can also be shown to contain a Sylow 2-subgroup $S$ of $G$, the possible structures of $S$ are completely determined; and now the possibilities for $G$ follow from various prior classification theorems for groups with such Sylow 2-groups. These latter results all concern groups of low 2-rank, a subject we shall discuss in considerable detail in the next chapter.

In the final case, Timmesfeld argues that $|A|=4$ and hence that $S$ is dihedral of order 8. Now the dihedral classification theorem yields that $G \cong A_{6}$ or $A_{7}$.

The theorem has a corollary, which is of fundamental importance for the study of groups of characteristic 2 type.

THEOREM 5. Let $G$ be a simple group and $A$ a nontrivial elementary 2-subgroup of $G$ with the following properties:

(a) $A$ is a T.I. set in $G$ (i.e. $A^{g} \cap A=1$ or $A$ for $g \in G$ ); and

(b) A centralizes no distinct conjugate of itself in $G$. (In particular, this is the case if $A$ is weakly closed in some Sylow 2-subgroup of $G$ containing $A$.)

Then $G$ is isomorphic to one of the groups listed in Theorem 3.

If $A$ is weakly closed and $A$ centralizes $A^{g}$ for $g \in G$, then $A A^{8}$ is a 2-group, whence $A A^{g} \leqslant S \in S y l_{2}(G)$ and so $A^{g}=A$ by the weak closure of $A$. Thus the second condition of $(b)$ implies the first.

We shall prove that the hypotheses of Theorem 3 follow from those of Theorem 5. Indeed, suppose by way of contradiction that $A$ is not weakly 
closed in $C_{a}$ for some $a \in A^{\#}$, in which case there is $B=A^{8} \leq C_{a}$ with $B \neq A$ for some $g \in G$. For $b \in B^{\#}, a \in A \cap A^{b}$, so $A=A^{b}$ as $A$ is a T.I. set by hypothesis. We conclude that $B$ normalizes $A$. Likewise as $A$ is a T.I. set and $B \neq A$, we have $B \cap A=1$. Set $C=A \cap N_{G}(B)$. Since $B$ normalizes $A$, it follows that $[C, B]<A \cap B=1$, so $B$ centralizes $C$. This forces $C<A$, otherwise $B$ centralizes $A$ and then $B=A$ by hypothesis, contradiction. Since $B C$ normalizes $B$, we conclude that $B C<B A$.

It follows now that there exists $x \in B A-B C$ with $x$ normalizing $B C$. Thus $B^{x} \leqslant(B C)^{x}=B C$. But $|B C|<|B A|=|B|^{2}$, as $A$ is conjugate to $B$. This forces $B \cap B^{x} \neq 1$, so $B^{x}=B$ as $B$ is a T.I. set. Hence $x \in N_{B A}(B)=$ $B C$, contrary to the choice of $x$.

10. Factorizations and 3 -groups. We shall limit the discussion to the prime 2 and shall write $\mathscr{Q}(S), J(S)$ for $\mathbb{Q}_{e}(S), J_{e}(S)$, respectively, for any 2-group $S$.

The factorization methods derive from the following basic result of Thompson [199].

Proposition 1. Let $X$ be a solvable group with $O(X)=1$ and let $S \in$ $\operatorname{Syl}_{2}(X)$. If $\Sigma_{3}$ is not involved in $X$ (in particular, if $X$ is a $3^{\prime}$-group), then we have

$$
X=C_{X}(Z(S)) N_{X}(J(S)) \text {. }
$$

We shall give the proof, which is quite elementary and very instructive, for it illustrates many ideas of local analysis-in particular, some used in the proof of Glauberman's $Z J$-theorem. Set $Z=Z(S)$. Since $X$ is 2-constrained (as $X$ is solvable) with trivial core, $Z \leq Z\left(O_{2}(X)\right)$ and hence $W \leqslant Z\left(O_{2}(X)\right)$, where $W$ is the normal closure of $Z$ in $X$. Since $Z\left(O_{2}(X)\right)$ is abelian, so therefore is $W$. Set $C=C_{X}(W)$ and $T=S \cap C$. Since $W \nabla X$, also $C \nabla X$, which implies that $T \in S y l_{2}(C)$. Also $C \leqslant C_{X}(Z)$ as $Z \leqslant W$.

It will suffice to prove that $J(S) \leqslant T$. Indeed, assume this is the case. Then $J(S)=J(T)$ char $T$ and so $N_{X}(T)$ normalizes $J(S)$. But $X=C N_{X}(T)$ by the Frattini argument as $T \in S y l_{2}(C)$, so $X=C N_{X}(J(S))=C_{X}(Z) N_{X}(J(S))$, as asserted.

We can therefore assume that $J(S) \star T$, whence there is $A \in \mathbb{Q}(S)$ such that $A \nless T$. Hence $A \nless C$. Set $\bar{X}=X / C$, so that $\bar{A} \neq 1$. We claim next that $O_{2}(\bar{X})=1$. Indeed, let $D$ be the preimage in $X$ of $O_{2}(\bar{X})$. Then $C \leqslant D \nabla X$ and so $D=C(S \cap D)$ as $D / C$ is a 2-group. But $S \cap D$ centralizes $Z=$ $Z(S)$ and hence so does $D$. Since $D \nabla X$, it follows that $D$ centralizes the normal closure $W$ of $Z$ in $X$. Since $C=C_{X}(W)$, this forces $D=C$ and so $1=\bar{D}=O_{2}(\bar{X})$, as claimed.

Let $\bar{F}=F(\bar{X})$, the Fitting subgroup of $\bar{X}$. By the previous paragraph, $\bar{F} \leqslant O(\bar{X})$. But $C_{\bar{X}}(\bar{F})<\bar{F}$ by Proposition 1.5.4, as $\bar{X}$ is solvable. Thus $\bar{A}$ does not centralize $\bar{F}$ and so $\vec{A}$ does not centralize $O_{q}(\bar{F})=O_{q}(\bar{X})$ for some (odd) prime $q$. Since $\bar{A}$ is an elementary abelian 2-group, one can prove easily by induction on the order of the group $O_{q}(\bar{X})$ that $\bar{A}$ normalizes, but does not centralize a subgroup $\bar{Y}$ of $O_{q}(\bar{X})$ of order $q$. Then $A u t(\bar{Y})$ is cyclic and so $\bar{B}=C_{\bar{A}}(\bar{Y})$ has index 2 in $\bar{A}$ with $\bar{Y} \bar{A} / \bar{B}$ a dihedral group of order $2 q$.

Now by definition of $C, \bar{Y}$ acts faithfully on $W$ and does not centralize $W$. Since $\bar{B} \bar{Y}=\bar{B} \times \bar{Y}$ acts on $W$ and $\bar{B}$ is a 2-group, the Thompson $A \times B$ - 
lemma implies that $\bar{Y}$ does not centralize $W_{0}=C_{W}(\bar{B})$. By standard properties of abelian groups ( $W_{0}$ is abelian), it follows that $\bar{Y}$ does not centralize $W_{1}=\Omega_{1}\left(W_{0}\right)$. Now set $V_{1}=\left[W_{1}, \bar{Y}\right]$, so that $V_{1} \neq 1$. Viewing $W_{1}$ as a vector space over $G F(2)$, it is immediate that $\bar{Y}$ has no nontrivial fixed points on $V_{1}$. Note also that as $\bar{A}$ normalizes both $\bar{Y}$ and $\bar{B}, A$ leaves $W_{0}, W_{1}$, and hence $V_{1}$ invariant.

Next, let $B$ be the subgroup of $A$ of index 2 which maps on $\bar{B}$. Then $B$ centralizes $V_{1}$ and so $B V_{1}$ is an elementary abelian subgroup of $S$. But $A \in \mathbb{Q}(S)$, so by definition of $\mathbb{Q}(S)$, we must have $m_{2}\left(B V_{1}\right) \leqslant m_{2}(A)$. This forces $B_{1}=B \cap V_{1}$ to be of index at most 2 in $V_{1}$.

Finally let $\bar{a} \in \bar{A}-\bar{B}$ and let $\bar{Y}=\langle\bar{y}\rangle$, so that $\bar{a}$ inverts $\bar{y}$. Thus $\langle\bar{a}, \bar{y}\rangle=$ $\left\langle\bar{a}, \bar{a}^{\bar{y}}\right\rangle$ is dihedral of order $2 q$. On the other hand, as $A$ is abelian $\bar{a}$ acts trivially on $B_{1}$, whence $\bar{a}^{\bar{y}}$ acts trivially on $B_{1}^{\bar{y}}$. But then $\left\langle\bar{a}, \bar{a}^{\bar{y}}\right\rangle$ and hence $\bar{Y}$ acts trivially on $B_{0}=B_{1} \cap B_{1}^{\bar{y}}$. Since $\bar{Y}$ has no nontrivial fixed points on $V_{1}$, this forces $B_{0}=1$. However, $\left|V_{1}: B_{1}\right| \leqslant 2$, so $\left|V_{1}: B_{0}\right| \leqslant 4$. We thus conclude that $\left|V_{1}\right|=4$. The only possibility therefore is $q=3$, whence $\langle\bar{a}, \bar{y}\rangle \cong$ $\Sigma_{3}$, contrary to our assumption that $\Sigma_{3}$ is not a section of $X$.

The assumption on $X$ is necessary, for let $X$ be the semidirect product of $S L\left(2,2^{n}\right)$ and its natural 2-dimensional module $V$ over $G F\left(2^{n}\right)$. (Note that $S L(2,2) \cong \Sigma_{3}$.) If $S \in S y l_{2}(X)$, one checks that $\mathscr{Q}(S)$ has exactly two members: namely, $V$ and a second member $A=Z(S) \times T$, where $Z(S)$ is a 1-dimensional subspace of $V$ (and hence of order $2^{n}$ ) and $T$ maps isomorphically on a Sylow 2-subgroup of $X / V \cong S L\left(2,2^{n}\right)$. Thus $J(S)=\langle V, A\rangle=S$ and it follows that $C_{X}(Z(S)) N_{X}(J(S)) \leqslant N_{X}(Z(S))$ (as $N_{X}(J(S))=N_{X}(S) \leqslant$ $N_{X}(Z(S))$ ). But clearly $Z(S)$ is not normal in $X$ (since $V$ is an irreducible $X$-module), so $X$ does not "factor".

Thompson established further factorizations of solvable groups. These depend upon the following definition.

Definition 2. If $S$ is a 2-group, let $\mathbb{Q}_{1}(S)$ be the set of elementary abelian 2-subgroups $B$ of $S$ such that either $B \in \mathbb{Q}(S)$ or $|B|=\frac{1}{2}|A|$, where $A \in$ $\mathcal{Q}(S)$, and set

$$
J_{1}(S)=\left\langle B \mid B \in \mathbb{Q}_{1}(S)\right\rangle .
$$

Arguing in the same way as in Proposition 1, Thompson proves [199].

Proposition 3. Let $X$ be a solvable group with $O(X)=1$ and let $S \in$ $\mathrm{Syl}_{2}(X)$. If $X$ does not involve $\Sigma_{3}$ or a dihedral group of order 10 (in particular, if $X$ has order prime to 3 and 5) then we have

$$
X=C_{X}(Z(S)) N_{X}\left(J_{1}(S)\right) \text {. }
$$

The semidirect product of a dihedral group of order 10 and its natural 4-dimensional module over $G F(2)$ is a counterexample to the proposition (as is $\Sigma_{3}$ on its natural 2-dimensional module).

Thompson's brilliance was shown here by his realization that yet a third factorization existed for $X$ and that these three factorizations together could be exploited.

Proposition 4. Let $X$ be a solvable group with $O(X)=1$ and let $S \in$ $S_{y} l_{2}(X)$. If $X$ does not involve $\Sigma_{3}$, then we have 


$$
X=N_{X}(J(S)) N_{X}\left(Z\left(J_{1}(S)\right)\right) .
$$

Corollary 5. If $X$ and $S$ are as in Proposition 3 and we set $N_{1}=$ $N_{X}(Z(S)), N_{2}=N_{X}(J(S)), N_{3}=N_{X}\left(Z\left(J_{1}(S)\right)\right)$, then for every $i, j, 1 \leqslant i$, $j \leqslant 3, i \neq j$, we have

$$
X=N_{i} N_{j} \text {. }
$$

The corollary follows at once from the three propositions together with the fact that $A B=B A$ whenever $A B$ is a group and that $N_{X}\left(J_{1}(S)\right) \leqslant$ $N_{X}\left(Z\left(J_{1}(S)\right)\right)=N_{3}$.

This is Thompson's "triple factorization lemma". Now comes his marvelous "three against two" argument [199].

Proposition 6. Let $G$ be a group and $H_{1}, H_{2}, H_{3}$ subgroups of $G$ such that for every permutation $\pi$ of the set $\{1,2,3\}$, we have

$$
H_{\pi(3)} \leqslant H_{\pi(1)} H_{\pi(2)} \text {. }
$$

Then $H_{i} H_{j}$ is a subgroup of $G$ for all $i, j, 1 \leqslant i, j \leqslant 3, i \neq j$.

Indeed, take $i=1, j=2$, for definiteness. We need only show that $\mathrm{H}_{2} \mathrm{H}_{1}$ $\leqslant H_{1} H_{2}$ to conclude that $H_{1} H_{2}$ is a group. But $H_{2} \leqslant H_{1} H_{3}$ and $H_{1} \leqslant H_{3} H_{2}$ by assumption, so $H_{2} H_{1} \leqslant\left(H_{1} H_{3}\right)\left(H_{3} H_{2}\right)=H_{1} H_{3} H_{2}$. But also $H_{3} \leqslant H_{1} H_{2}$, whence $H_{1} H_{3} H_{2} \leqslant H_{1}\left(H_{1} H_{2}\right) H_{2}=H_{1} H_{2}$. Thus $H_{2} H_{1} \leqslant H_{1} H_{2}$, as required.

As remarked earlier, Thompson developed these ideas in connection with the odd order problem. We illustrate their significance by proving the following result.

THEOREM 7. If $G$ is a simple group in which every 2-local subgroup has order prime to both 3 and 5 , then $G \cong L_{2}\left(2^{n}\right), n$ odd, $n \geqslant 1, U_{3}\left(2^{n}\right), n \neq 0(\bmod 3)$, or $\operatorname{Sz}\left(2^{n}\right), n$ odd, $n \geqslant 3$.

Let $G$ be a minimum counterexample. We shall argue first that every 2-local subgroup of $G$ is solvable with trivial core, at which point Thompson's ideas will be immediately applicable. By assumption, every 2-local subgroup $H$ of $G$ has order prime to 3 and 5 . If $H$ is nonsolvable, then $H$ has a nonabelian simple section $K$ and $|K|$ is prime to 3 and 5. Clearly then $K$ satisfies the hypothesis of the theorem, so by the minimality of $G, K \cong L_{2}\left(2^{n}\right)$, $U_{3}\left(2^{n}\right)$, or $S z\left(2^{m}\right)$ for suitable $m$. But then 3 or 5 divides $|K|$, contradiction. We conclude that every 2-local subgroup of $G$ is solvable.

We claim next that a Sylow 2-subgroup of $G$ is connected and $m_{2}(G) \geqslant 3$. Assume false, whence $r_{2}(G) \leqslant 4$ by Corollary I.5.18. Since $G$ is simple, Frobenius' normal complement theorem implies that some 2-local subgroup $H$ of $G$ does not have a normal 2-complement. Choose $H$ so that a Sylow 2-subgroup $S$ of $H$ has maximal order. Set $\bar{H}=H / O(H), \bar{R}=O_{2}(\bar{H})$ and $\tilde{R}=\bar{R} / \phi(\bar{R})$. Since $H$ is solvable, $C_{\bar{H}}(\bar{R}) \leqslant \bar{R}$ and hence $C_{\bar{H}}(\tilde{R}) \leqslant \bar{R}$ by Proposition I.4.3. Thus $\bar{H} / \bar{R}$ is isomorphic to a subgroup of $\operatorname{Aut}(\tilde{R})$. But $\tilde{R}$ is elementary abelian by Proposition I.4.3 and so has rank at most 4, since $r_{2}(G) \leqslant 4$. Thus $\operatorname{Aut}(\tilde{R}) \leqslant L_{4}(2) \cong A_{8}$. Hence $\bar{H} / \bar{R}$ is a nontrivial solvable subgroup of $A_{8}$ of order prime to 3 and 5 with $O_{2}(\bar{H} / \bar{R})=1$. The only possibility is that $\bar{H} / \bar{R}$ is of order 7. In particular, $\bar{R}=\bar{S}$.

Let $R$ be the inverse image of $\bar{R}$ in $H$. Then $R \nabla H$ as $\bar{R} \nabla \bar{H}$ and 
$R=O(H) S$. Hence by the Frattini argument, $H=R N_{H}(S)=O(H) N_{H}(S)$. Since $H$ does not have a normal 2-complement, neither therefore does $H_{1}=N_{G}(S)$. By maximality of $S, S \in S y l_{2}\left(H_{1}\right)$, whence $S \in S y l_{2}(G)$. On the other hand, $\bar{H} / \bar{R}=\bar{H} / \bar{S}$ induces a group of automorphisms of $\bar{S}$ of order 7 and using this condition, we can argue without much difficulty that $\bar{S}$ must be connected group and $m_{2}(\bar{S})>3$. Thus $S$ is connected and $m_{2}(G)>$ 3 , as asserted.

Now we can apply Theorem I.5.19 and conclude that every 2-local subgroup of $G$ has a trivial core.

Now let $S \in S y l_{2}(G)$ and set $N_{1}=N_{G}(Z(S)), N_{2}=N_{G}(J(S))$, and $N_{3}=$ $N_{G}\left(Z\left(J_{1}(S)\right)\right)$. Then each $N_{i}$ is a 2-local subgroup of $G$ and so is solvable of order prime to 3 and 5 with trivial core, $1 \leqslant i \leqslant 3$. Since also $S \in S y l_{2}\left(N_{i}\right)$, applying Corollary 5 to the groups $N_{i}$, we obtain

$$
N_{\pi(3)} \leqslant N_{\pi(1)} N_{\pi(2)}
$$

for every permutation $\pi$ of the set $\{1,2,3\}$. Hence by Proposition $6, M=$ $N_{1} N_{2}$ is a subgroup of $G$.

Suppose $M=G$. Then any element $g \in G$ has the form $g=g_{1} g_{2}$ with $g_{i} \in N_{i}, i=1,2$. But then

$$
O_{2}\left(N_{1}\right)^{g}=O_{2}\left(N_{1}\right)^{g_{1} g_{2}}=O_{2}\left(N_{1}\right)^{g_{2}}<S^{g_{2}} \leqslant N_{2}
$$

(as $\left.O_{2}\left(N_{1}\right) \leqslant S<N_{2}\right)$. Thus the normal closure of $O_{2}\left(N_{1}\right)$ in $G$ is contained in $N_{2}$. However, as $O_{2}\left(N_{1}\right) \neq 1$ and $N_{2}<G$, this contradicts the simplicity of $G$. We therefore conclude that $M<G$.

Finally we argue that $M$ is strongly embedded in $G$. It will suffice to show that $M$ contains the normalizer of every nonidentity subgroup of $S$. Suppose false and let $H$ be a 2-local subgroup of $G$ such that $H \star M$ with $Q=S \cap$ $H$ of largest possible order. Clearly $Q \neq 1$. As in the proof of Theorem 6.7, $Q \in \mathrm{Syl}_{2}(H)$. This time we use Proposition 1 rather than the $Z J$-theorem and conclude that

$$
H=N_{H}(Z(Q)) N_{H}(J(Q)) .
$$

If $Q=S$, then by (3), $H \leqslant N_{1} N_{2}=M$, contrary to the choice of $H$, so $Q<S$. But then $N_{S}(Z(Q))>Q$ and $N_{S}(J(Q))>Q$, so by the maximality of $Q$, we have $N_{G}(Z(Q))<M$ and $N_{G}(J(Q))<M$. Thus again $H \leqslant M$ by (3), contradiction.

Thus $M$ is strongly embedded, as asserted, and so by Bender's theorem, $G \cong L_{2}\left(2^{n}\right), n>2, U_{3}\left(2^{n}\right), n \geqslant 2$, or $S z\left(2^{n}\right), n$ odd, $n \geqslant 3$. The hypothesis on the orders of 2-locals now forces $n$ to satisfy the restrictions of the theorem. Thus $G$ is not a counterexample and the theorem is proved.

In attempting to generalize these factorization results to nonsolvable groups $X$ with $F^{*}(X)$ a 2-group, two distinct problems arise. First, in the proof of Proposition 1 (and likewise in the proof of Proposition 3), the given arguments will again reduce to the module action of a group $\bar{A} \bar{Y} / \bar{B}$ on a vector space $V_{1}$ over $G F(2)$ with $\bar{B}$ centralizing $\bar{Q}$ and $V_{1}$ and with $V_{1}=\left[V_{1}, \bar{Y}\right]$. However, now $\bar{Y}$ need not be a cyclic group, but $\bar{Y}$ may also be a quasisimple group, isomorphic to a homomorphic image of a component of $L(\bar{X})$. However, in the latter case, $\bar{B}=C_{\bar{A}}(\bar{Y})$ need not be of index 2 in $\bar{A}$. The 
validity of the factorization of $X$ depends upon the nature of the action of $\bar{Y}$ on this module $V_{1}$ and in turn reduces to considerations about the dimension of the fixed point space $B_{1}=A \cap V_{1} \leqslant C_{V_{1}}(A)$.

The discussion above with $Y \cong S L\left(2,2^{n}\right)$ and $V_{1}$ the natural module provides a counterexample to any proposed factorization for groups involving $S L\left(2,2^{n}\right)$. For the general analysis of groups of characteristic 2 type, it is very important to know precisely which possibilities for the pair $\bar{Y}, V_{1}$ will lead to a factorization and which will not ( $\bar{Y}$ being assumed to be a $K$-group). Such "failures of factorization" will be described in detail in the next section. Let me just say here that this phenomenon is very much associated with the groups of Lie type of characteristic 2 and the alternating groups, factorization essentially always holding if $Y$ is not of one of these forms, no matter what module $V_{1}$ one takes.

On the other hand, the groups $S z\left(2^{n}\right)$ are somewhat special since they have order prime to 3 and are the only known simple groups with this property. Thompson was able to extend Proposition 1 to this case by analyzing the $G F(2)$-modules for $S z\left(2^{n}\right)$ [204].

Proposition 8. Let $X$ be a $K$-group of order prime to 3 in which $F^{*}(X)$ is a 2-group. If $S \in S y l_{2}(X)$, then we have

$$
X=C_{X}(Z(S)) N_{X}(J(S)) \text {. }
$$

In view of the dihedral group of order 10 counterexample, one cannot prove the corresponding $Z, J_{1}$ factorization for an arbitrary such group $X$.

The third $J, Z J_{1}$ factorization is rare for nonsolvable groups. Indeed, in the proof of any such result, one again reduces (as in the proof of Proposition 1) to an $\bar{A} \bar{Y} / \bar{B}$ situation for some $A \in \mathbb{Q}(S)$ and again considers the largest subgroup $B$ of $A$ which maps on $\bar{B}$. For the ensuing argument, it is essential that $B$ be an element of $\mathcal{Q}_{1}(S)$. However, this will be the case if and only if $|A: B| \leqslant 2$ and hence if and only if $|\bar{A}: \bar{B}|<2$. But $\bar{Y}$ may be a simple group in which case $m_{2}(\bar{Y}) \geqslant 2$ and $\bar{A} / C_{\bar{A}}(\bar{Y})$ may be noncyclic. Hence one cannot assert, in general, that $\bar{B}$ has codimension at most 1 in $\bar{A}$; some additional restriction is necessary to reach such a conclusion.

Some time after completing the classification of $N$-groups, Thompson undertook the task of proving that the Suzuki groups $S z\left(2^{n}\right)$ were, in fact, the only simple groups of order prime to 3 [204]. Using standard signalizer functor methods, Thompson showed that a minimal counterexample $G$ to such a theorem was of characteristic 2 type. This effort could not be completely routine since the Rudvalis group $R u$ has $S z(8) \times Z_{2} \times Z_{2}$ as the centralizer of one of its involutions. Even though $R u$ is not a $3^{\prime}$-group, this fact is not "visible" from this centralizer.

Now every proper subgroup of $G$ is a $K$-group and so its nonsolvable composition factors are necessarily Suzuki groups. For some time Thompson sought a triple factorization for the 2-local subgroups of $G$, which would enable him to emulate the proof of Theorem 7. Unfortunately he never found one and was therefore forced to resort to the full panoply of techniques and the major subdivisions $e(G) \geqslant 3, e(G)=2$, and $e(G)=1$ of the $N$-group analysis to complete the classification of simple 3 '-groups. 
However, by introducing an ingeniously conceived subgroup $\hat{J}(S)$ to replace $J_{1}(S)$, Glauberman has recently succeeded in proving a triple factorization for $K$-groups $X$ of order prime to 3 in which $F^{*}(X)$ is a 2-group (and for more general groups $X$ which do not involve $\Sigma_{4}$ ) [75].

Definition 9. Let $T$ be a 2-group. An elementary abelian normal subgroup $V$ of $T$ is said to be restricted in $T$ provided that, for any elementary abelian 2-subgroup $R$ of $T / C_{T}(V)$, we have

$$
\left|V / C_{V}(R)\right|>|R|^{3 / 2} \text { and }|[V, R]|>|R| .
$$

Furthermore, $T$ is said to be an $E$-group if $T$ has no restricted subgroups.

The following lemma of Glauberman shows that the notion of $E$-group is not vacuous.

LEMMA 10. For any 2-group $S, J(S)$ is an E-group.

Now we can define $\hat{J}(S)$.

Definition 11. For any 2-group $S$, set

$$
\hat{J}(S)=\langle T| J(S) \leqslant T \leqslant S \text { and } T \text { is an } E \text {-group }\rangle .
$$

Thus $J(S) \leqslant \hat{J}(S)$. (Note that by the definitions, likewise $J(S) \leqslant J_{1}(S)$.)

We remark that the groups $S z\left(2^{n}\right)$ and $U_{3}\left(2^{n}\right)$ are the only simple $K$-groups with nonabelian Sylow 2-groups which do not involve $\Sigma_{4}$. Thus Glauberman's factorizations deal with 2-constrained groups whose only nonsolvable composition factors are of one of these types or else have abelian Sylow 2-subgroups.

THeOREM 12. Let $X$ be a $K$-group with $F^{*}(X)$ a 2-group such that $X$ does not involve $\Sigma_{4}$. If $S \in S y l_{2}(X)$, then we have

(i) $X=C_{X}\left(\Omega_{1}(Z(S))\right) N_{X}(J(S))$;

(ii) $X=C_{X}\left(\Omega_{1}(Z(S))\right) N_{X}(\hat{J}(S))$; and

(iii) $X=C_{X}\left(\Omega_{1}(Z(J(S)))\right) N_{X}(J(S))$.

Glauberman's proof is a tour de force of delicate commutator calculations.

On the basis of the theorem, Glauberman readily obtains the following extension of Thompson's 3'-theorem [75].

THEOREM 13. If $G$ is a simple group with nonabelian Sylow 2-subgroups which does not involve $\Sigma_{4}$, then $G \cong S z\left(2^{n}\right)$ or $U_{3}\left(2^{n}\right), n$ odd.

Taking $G$ to be a minimal counterexample, Glauberman could have argued exactly as in Theorem 7 that $G$ has a strongly embedded subgroup, provided he first showed that $G$ had to be of characteristic 2 type. However, he was able to avoid even that amount of detailed analysis by appealing to Goldschmidt's strongly closed abelian subgroup theorem rather than to Bender's strongly embedded theorem. Thus he obtained Theorem 13 from the following fusion result together with Goldschmidt's classification theorem (Theorem 7.3).

Proposition 14. Set $A=\left\langle\Omega_{1}(Z(S))^{x} \mid x \in N_{G}(J(S))\right\rangle$. Then $A$ is a nontrivial elementary abelian subgroup of $S$ and $A$ is strongly closed in $S$ with respect to $G$. 
The point here is that even though $G$ need not be of characteristic 2 type, Goldschmidt's extension of Alperin's fusion theorem (Theorem 5.2) shows that 2-fusion is determined entirely by 2-constrained 2-local subgroups $H$ of $G$ such that $O_{2}(H)$ maps onto $O_{2}(H / O(H))$. Since any such 2-local $H$ is a $K$-group and does not involve $\Sigma_{4}$, it follows that $H$ has a triple factorization. Exploiting this fact, Glauberman establishes the strong closure of $A$.

11. Failure of factorization. As we have seen in the preceding section, the question of whether a group $X$ with $F^{*}(X)$ a 2-group satisfies either the $(Z, J)$ - or $\left(Z, J_{1}\right)$-factorization with respect to a Sylow 2-subgroup $S$ of $X$ reduces to a module statement for a certain section $\bar{A} \bar{Y} / \bar{B}$ of $X$, where $\bar{Y}$ is a quasisimple group with $O_{2}(\bar{Y})=1$. Examining the picture a little more closely, one can rephrase these factorization questions in general terms.

If $V$ is a faithful $G F(2)$-module for the group $Y$ with $O_{2}(Y)=1$, does $Y$ contain a nontrivial elementary abelian 2-subgroup $A$ such that

$$
m_{2}(A) \geqslant m_{2}\left(V / C_{V}(A)\right)
$$

or such that

$$
m_{2}(A) \geqslant m_{2}\left(V / C_{V}(A)\right)-1 ?
$$

In practice, we are concerned primarily with the case in which $F^{*}(Y)$ is a quasisimple $K$-group (taking $\bar{A} \bar{Y} / \bar{B}$ above as $Y$, one has $F^{*}(Y)$ quasisimple).

DEFINITION 1. Let $Y$ be a group with $F^{*}(Y)$ quasisimple and $O_{2}(Y)=1$. If $V$ is a faithful $G F(2)$-module for $Y$, we say that $(Y, V)$ is an $F$-pair if $Y$ contains a subgroup $A$ satisfying (1) and that $(Y, V)$ is an $F_{1}$-pair if $Y$ contains a subgroup $A$ satisfying (2). Any such subgroup $A$ is called a nonfactor of $(Y, V)$.

Obviously if $(Y, V)$ is an $F$-pair, it is an $F_{1}$-pair. If $(Y, V)$ is an $F_{1}$-pair, but not an $F$-pair, then for any nonfactor $A$ of $(Y, V)$, we must have

$$
m_{2}(A)=m_{2}\left(V / C_{V}(A)\right)-1 .
$$

Note that if $(Y, V)$ is an $F$-pair with $|A|=2$, then $m_{2}\left(A / C_{V}(A)\right)=1$ and so $A$ centralizes a hyperplane of $V$. In other words, the involution of $A$ is a transvection on $V$.

One of the first results in this direction is due to McLaughlin, who classified all groups which are generated by their transvections [147].

THEOREM 2. Let $V$ be an irreducible module of dimension $n$ over $G F(2)$ for the group $Y$. If $Y$ is generated by its transvections, then $Y \cong S L(n, 2), S p(n, 2)$, $S O^{ \pm}(n, 2), \Sigma_{n+1}$ or $\Sigma_{n+2}$ with $n \geqslant 4$ except in the first case. (Also $n>4$ when $Y=S O^{+}(n, 2)$.)

To prove the theorem, McLaughlin builds up the geometries of the classical and symmetric groups from properties of the action of the transvections on $V$. Note that as $V$ is defined over $G F(2)$, the subgroup of $Y$ generated by a transvection is a root subgroup, so the argument can be viewed as in the same spirit as proofs of quadratic pair and Fischer-Timmesfeld type theorems.

Aschbacher has proved the following general result [11]. 
THEOREM 3. Let $(Y, V)$ be an $F_{1}$-pair in which $L=F^{*}(Y)$ is a quasisimple $K$-group. Then one of the following holds:

(i) $L \in$ Chev(2);

(ii) $L / Z(L) \cong A_{n}$ for some $n$;

(iii) $|Z(L)|=3, L / Z(L) \cong U_{4}(3)$ or $M_{22}$ and $(Y, V)$ is not an F-pair; or

(iv) $L \cong M_{22}, M_{23}$, or $M_{24}$, and $(Y, V)$ is not an $F$-pair.

The theorem definitely shows that failure of factorization is a characteristic 2 and alternating group phenomenon. Detailed properties of the groups of Lie type of odd characteristic and of the sporadic groups are required to prove that the groups in (iii) and (iv) are the only such candidates for $Y$.

Aschbacher also analyzes the alternating case in considerable detail, determining the possible modules and embedding of nonfactors in $Y$ under various conditions. In particular, he establishes the following result.

Proposition 4. If $(Y, V)$ is an F-pair with $Y=A_{n}$ or $\Sigma_{n}, n>9$, and $V$ is an irreducible $Y$-module, then $V$ is the natural module for $Y$.

Here the natural modules for $A_{n}$ and for $\Sigma_{n}$ are defined as follows. Let $X$ be a split extension of $E \cong E_{2^{n}}$ by $\Sigma_{n}$, in which the action of an element of $\Sigma_{n}$ on $E$ is determined by its corresponding permutation action on a fixed basis $x_{1}$, $x_{2}, \ldots, x_{n}$ of $E$. Clearly $\Sigma_{n}$ fixes the element $x=x_{1} x_{2} \cdots x_{n}$ of $E$ and also leaves invariant the subspace $E_{0}=\left\langle x_{i}-x_{j} \mid 1 \leqslant i, j \leqslant n\right\rangle$. If $n$ is even, then $\langle x\rangle\left\langle E_{0}<E\right.$ (with $\left|E: E_{0}\right|=2$ ) and $V=E_{0} /\langle x\rangle$ is the natural module for $\Sigma_{n}$ (and $V$ is of dimension $n-2$ ). On the other hand, if $n$ is odd, then $E=E_{0} \times\langle x\rangle$ and $V=E_{0}$ is the natural module for $\Sigma_{n}$ (and $V$ is of dimension $n-1)$. Thus in either case the natural module for $\Sigma_{n}$ is the unique nontrivial composition factor of $E$ as a $\Sigma_{n}$-module. The restriction of $V$ to $A_{n}$ is the natural representation of $A_{n}$.

Cooperstein and G. Mason [45], [46] have determined the possible $F$-pairs $(V, Y)$ when $Y \in C h e v(2)$. Their analysis depends on the general theory, worked out by Steinberg and Curtis [186], [223], of the irreducible $G F(p)$-representations of the groups of Lie type of characteristic $p$. These representations are all described as suitable tensor products of so-called "fundamental" irreducible representations. The fundamental representations are in turn in one-one correspondence with certain well-defined sets of "weight" vectors in Euclidean $\mathbf{R}^{n}$ space, these weights themselves determined by the root system of the associated Lie algebra. We need not be more explicit, since the final result of Cooperstein and Mason's investigations can be phrased in more classical terminology, not involving the Lie theory machinery. Furthermore, as in the alternating case, there are some low dimensional exceptions, which we shall not attempt to describe here.

THEOREM 5. Let $(Y, V)$ be an F-pair with $Y$ of Lie type defined over $G F(q)$, $q=2^{m}$, and $V$ irreducible as a $Y$-module. Then we have

(i) If $Y=S L(n, q)$, then $V$ is either the standard module of dimension $n$ over $G F(q)$, the second exterior product of the standard module, or $V$ is the dual of one of these two modules.

(ii) If $Y=S U(n, q), n \geqslant 5$, then $V$ is the standard module over $G F\left(q^{2}\right)$.

(iii) If $Y=S O^{ \pm}(n, q), n \geqslant 11$, then $V$ is the standard module over $G F(q)$.

(iv) If $Y=G_{2}(q)$, then $V$ is the 6-dimensional symplectic module over $G F(q)$. 
The other exceptional groups do not occur as possibilities for $Y$ in an $F$-pair. Neither does the group $S U(3, q)$. Note also that if $V$ is a $Y$-module over $G F(q), q=2^{n}$, then $V$ is also a $Y$-module over $G F(2)$ (as required by the definition of an $F$-pair).

If $V$ is the natural module for $S L(n, q)$, the second exterior product of $V$ is defined to be the subspace of the tensor product $V \otimes V$ spanned by all vectors of the form $v \otimes w-w \otimes v$ for $v, w \in V$. This subspace is invariant under $S L(n, q)$ and so is an $S L(n, q)$-module. Furthermore, the standard module for $S U(n, q)$ is simply the restriction to $S U(n, q)$ of the standard module for $S L\left(n, q^{2}\right)$ over $G F\left(q^{2}\right)$. The standard module $V$ for $S O^{ \pm}(n, q)$ is the restriction of the standard module $V$ for $S L(n, q)$ to the subgroup leaving invariant an appropriate nondegenerate quadratic form on $V$. Finally the group $G_{2}(q)$ is always a subgroup of $B_{3}(q)$, which in characteristic 2 is isomorphic to $C_{3}(q)$ and so $G_{2}(q)$ is a subgroup of $S p(6, q)$. Thus when $q=2^{m}, G_{2}(q)$ acts irreducibly on a 6-dimensional symplectic space over $G F(q)$ and this is the module referred to in (iv) above.

With sufficient effort, one could undoubtedly determine the possible irreducible modules for $F_{1}$-pairs $(Y, V)$ with $F^{*}(Y)$ a quasisimple $K$-group by the same general methods as were used in establishing the above results.

If $X$ is a group with $F^{*}(X)$ a 2-group and the $(Z, J)$-factorization fails with respect to $S \in S y l_{2}(X)$, the natural question to ask is the following: What is the structure of the normal closure $N$ of $J(S)$ in $X$ ?

Obviously failure of factorization implies that $J(S) \$ O_{2}(X)$, so $N \$$ $O_{2}(X)$. Glauberman has given a complete answer in the solvable case [225].

THeOReM 6. Let $X$ be a solvable group with $F^{*}(X)$ a 2-group, let $S \in$ Syl $l_{2}(X)$, and assume that $J(S) \nless O_{2}(X)$. Set $N=\left\langle J(S)^{X}\right\rangle, \quad V=$ $\Omega_{1}\left(Z\left(O_{2}(X)\right)\right)$, and $\bar{X}=X / O_{2}(X)$. Then we have

(i) $\bar{N}=\bar{N}_{1} \times \bar{N}_{2} \times \cdots \times \bar{N}_{r}$, where each $\bar{N}_{i} \cong \Sigma_{3}, 1 \leqslant i \leqslant r$.

(ii) If $V_{i}=\left[V, N_{i}\right], 1 \leqslant i \leqslant r$, then each $V_{i} \cong Z_{2} \times Z_{2}$ and $[V, N]=V_{1} \times$ $V_{2} \times \cdots \times V_{r}$.

In view of this result, there is a natural conjecture when the solvability assumption is dropped:

The group $\bar{N}$ is the direct product of subgroups $\bar{N}_{i}$ of Lie type of characteristic 2 or alternating or symmetric groups, $1<i<r$; and (modulo the fixed points of $\bar{N}$ on $[V, \bar{N}])[V, \bar{N}]$ is the direct product of irreducible $\bar{N}_{i}$ modules $V_{i}$ such that $\left(\bar{N}_{i}, V_{i}\right)$ is an $F$-pair, $1 \leqslant i \leqslant r$.

Easy examples show that one cannot expect $[V, \bar{N}]$ itself to be such a direct product.

For the applications, one would need this conjecture only under the additional assumption that

$$
\left\langle C_{X}\left(\Omega_{1}(Z(S))\right), N_{X}(J(S))\right\rangle<X .
$$

This is a stronger condition than simply failure of $(Z, J)$-factorization. However, even this weaker conjecture appears to be difficult. Aschbacher has obtained a partial solution, which is closely related to the pushing up results we shall discuss in the next section [12]. For simplicity we state only a special case of his theorem. 
THEOREM 7. Let $X$ be a group with $F^{*}(X)$ a 2-group, let $S \in S y l_{2}(X)$, set $V=\Omega_{1}\left(Z\left(O_{2}(X)\right)\right)$, and $\bar{X}=X / O_{2}(X)$. Assume the following two conditions:

(a) $X_{0}=\left\langle C_{X}\left(\Omega_{1}(Z(S))\right), N_{X}(J(S))\right\rangle<X$; and

(b) If $A, B \in \mathbb{Q}(S)$ with $\bar{B} \leqslant \bar{A}$, then either $\bar{B}=\bar{A}$ or $\bar{B}=1$.

Then we have

(i) $X=N X_{0}$, where $N \nabla X$ and $\bar{N}$ is the direct product of $\bar{X}$-conjugate subgroups $\bar{N}_{i}, 1 \leqslant i \leqslant r$;

(ii) Either $\bar{N}_{i} \cong S \underline{L}\left(n, 2^{m}\right)$ for some $m$ and $n$ or $\bar{N}_{i} \cong \Sigma_{n}, n$ odd; and

(iii) If $U=[V, \bar{N}] / C_{[V, \bar{N}]}(\bar{N})$ and $U_{i}=\left[U, \bar{N}_{i}\right], 1 \leqslant i \leqslant r$, then $U_{i}$ is a natural $\bar{N}_{i}$-module and $U=U_{1} \times U_{2} \times \cdots \times U_{r}$.

Note that Aschbacher does not assume that $X$ is a $K$-group. In the course of the argument he is able to identify the groups $\bar{N}_{i}$ either from McLaughlin's theorem above or from Timmesfeld's results on root involutions.

12. Pushing up theorems. Let $G$ be a group of characteristic $p$ type and $H$ a $p$-local subgroup of $G$ which does not contain a Sylow $p$-subgroup of $G$. Pushing up theorems are concerned with the following general question: Under what conditions can one assert that $H$ is contained in a $p$-local subgroup $H^{*}$ of $G$ whose Sylow $p$-subgroups have larger order than those of $H$ ?

Here we shall consider only the case $p=2$ and we begin with a slightly more restricted situation. Suppose $G$ is simple with Sylow 2-subgroup $S$ and assume that $S$ is contained in a unique maximal 2-local subgroup $M$ of $G$. We ask the following natural question:

Must $M$ be strongly embedded in $G$ under these conditions?

Proceeding by contradiction as we did in $\$ 7$, one quickly reduces the problem to the following more precise situation:

(a) $G$ contains a maximal 2-local subgroup $X$ such that $X \nless M$

and $T=S \cap X \in S y l_{2}(X) ;$ and

(b) If $H$ is a 2-local subgroup of $G$ such that $|S \cap H|>|T|$, then $H \leqslant M$.

In view of the assumed uniqueness of $M$, (a) implies that $T<S$. Furthermore, by (b), if $R$ is any nontrivial normal subgroup of $T$ such that $N_{S}(R)>T$, (in particular, if $R$ char $T$ ), then $N_{G}(R) \leqslant M$ and so $N_{X}(R) \leqslant$ $M$. Thus we have

$$
\left\langle N_{X}(R) \mid 1 \neq R \nabla T, N_{X}(R)>T\right\rangle\langle X .
$$

On the other hand, as $X$ is a maximal 2-local subgroup and $G$ is simple, we also obviously have

$$
\left\langle N_{G}(R) \mid 1 \neq R \leqslant T, R \nabla X\right\rangle=X .
$$

Hence we are reduced to analyzing the structure of a 2-local subgroup $X$ of $G$ satisfying conditions (3) and (4).

This type of problem first occured in Sims' study of primitive permutation groups in which a one-point stabilizer has an orbit of length three [171]. This is a very tight situation, as the following result clearly indicates. 
Proposition 1. If $G$ is a primitive permutation group in which the one-point stabilizer $X$ has an orbit of length 3 , then we have

(i) $G=\left\langle X, X^{g}\right\rangle$ for some $g \in G$;

(ii) $\left|X: X \cap X^{g}\right|=3$; and

(iii) $X / O_{2}(X) \cong \Sigma_{3}$. In particular, $|X|=2^{a} \cdot 3$.

Sims' main result asserts the following:

THEOREM 2. If $G$ is a primitive permutation group on a set in which the one-point stabilizer $X$ has an orbit of length 3 , then $|X|=2^{a} \cdot 3$, where $a \leqslant 4$.

It is in this paper that Sims' ideas about groups and their associated graphs are spelled out. In particular, the proof of Theorem 2 is carried out by analyzing the graph $\Gamma$ determined from an orbit $\Delta$ of $X$ of length $3, \Gamma$ being an example of a cubic graph. Such graphs were investigated by Tutte [232], [233] and the particular case of the theorem in which $\Delta$ is "self-paired" is a direct consequence of his results. (Self-pairing means that if $g \in G, a \in \Delta$, and $a^{g^{-1}} \in \Delta$, then also $a^{g} \in \Delta$.) Sims' proof in the general case involves an extension of Tutte's argument.

Using Sims' results, Wong completed the classification permutation groups in which the one-point stabilizer $X$ has an orbit of length 3 [221]. Indeed, using Sims' bound on $|X|$, he first showed quite directly that

$$
X \cong \Sigma_{3}, \Sigma_{3} \times Z_{2}, \Sigma_{4}, \text { or } \Sigma_{4} \times Z_{2} \text {. }
$$

In the first case, $G$ has a self-centralizing subgroup of order 3 and a theorem of Feit and Thompson [224] applies to yield the possibilities for $G$. In the second and third cases, $G$ has dihedral or quasi-dihedral Sylow 2-subgroups and $G$ is determined from the classification theorems for such groups. In the final case, Wong first pins down the possible structures of a Sylow 2-subgroup of $G$ (of order $2^{4}$ or $2^{5}$ ). In particular, $G$ has a normal subgroup of index 2 and again $G$ is determined from prior classification theorems.

The final result is as follows.

THEOREM 3. If $G$ is a primitive permutation group in which the one-point stabilizer has an orbit of length 3 , then $G \cong A_{5}, \Sigma_{5}, P G L(2,7), L_{2}(11), L_{2}(q)$, $q \equiv \pm 1(\bmod 16), L_{3}(3)$, or $\operatorname{Aut}\left(L_{3}(3)\right)$.

Glauberman made a serious attempt to place the Tutte-Sims argument in a general framework. The following is a particular case of his first effort [74].

THEOREM 4. Let $X$ be a 2-local subgroup of the group $G$ such that $F^{*}(X)=$ $\mathrm{O}_{2}(X)$ and $X / \mathrm{O}_{2}(X)$ is a dihedral group. If no 2-local subgroup of $G$ contains $X$ properly, then either $X$ contains a Sylow 2-subgroup of $G$ or $X \cong \Sigma_{4}$ or $\Sigma_{4} \times Z_{2}$.

If $T \in \operatorname{Syl}_{2}(X)$, then the hypotheses imply that (4) holds. Hence the proof of Theorem 4 (and Sims' theorem as well) involves an analysis of (4) in the special case that $X / O_{2}(X)$ is a dihedral group.

Conditions (3) and (4) depend not only on the embedding of $T$ in $X$, but also on that of $X$ in $G$, which makes for very complicated configurations. It is therefore desirable to attempt to study the structure of $X$ as an independent 
problem, free from its embedding in the larger group $G$. The most direct way to accomplish this and still retain the essential features of the given situation is to restrict oneself to characteristic subgroups of $T$ in place of normal subgroups. This suggests the following definition.

Definition 5. For any group $X$ and Sylow 2-subgroup $T$ of $X$, the characteristic generated core $C(X ; T)$ of $X$ is defined to be

$$
\left.C(X ; T)=\left\langle N_{X}(R)\right| 1 \neq R \text { char } T\right\rangle .
$$

Clearly $C(X ; T)$ is determined up to conjugacy by the Sylow 2-subgroup $T$ of $X$.

Thus the "characteristic analogue" of (3) is simply the statement that $C(X ; T)<X$. Clearly this implies that no nontrivial characteristic subgroup of $T$ is normal in $X$, the latter assertion being the corresponding characteristic analogue of (4).

We can express the general problem in the following precise way.

If $X$ is a group with $C(X ; T)<X$ for $T \in S y l_{2}(X)$, describe the normal subgroup $N$ of $X$ which is minimal subject to the condition $X=N C(X ; T)$.

The subgroup $N$ gives a measure of the extent to which $C(X ; T)$ fails to generate $X$. Since $Z(T)$ and $J(T)$ are characteristic subgroups of $T$, we certainly have

$$
\left\langle C_{X}\left(\Omega_{1}(Z(T))\right), N_{X}(J(T))\right\rangle<C(X ; T) ;
$$

so if $C(X ; T)<X$, it follows that the $(Z, J)$-factorization necessarily fails for $X$. Hence, in particular, the results of the previous section apply to $X$.

For the applications, one is interested in both local and global answers to (6). The local problem is concerned with the case in which $F^{*}(X)$ is a 2-group and the global problem with the case that $X$ itself is a simple group of characteristic 2 type (with all proper subgroups $K$-groups). Clearly $N=X$ in the global case, and we are asking then for a classification of finite simple groups $X$ of characteristic 2 type with $C(X ; T)<X, T \in S y l_{2}(X)$. Strictly speaking, "pushing up" refers only to the local case and so we shall limit the present discussion to that situation. The global problem will be discussed in Chapters VII and VIII.

Glauberman has treated the case that $X / O_{2}(X)$ is a dihedral group in [74] and Aschbacher [13], Baumann [17] and Niles [149] have independently analyzed the general case in which $X / O_{2}(X) \cong L_{2}\left(2^{n}\right)$ for some $n$ (the case $n=1$ corresponding to the dihedral case). Aschbacher considered the special case in which $X$ is a 2-local subgroup of a group of characteristic 2 type. (Niles also treated the analogous problem for odd primes. It should also be noted that Glauberman and Sims also obtained results for arbitrary primes.)

The Baumann-Niles theorem asserts the following.

THEOREM 6. Let $X$ be a group with $F^{*}(X)=O_{2}(X)$ and $X / O_{2}(X) \cong L_{2}\left(2^{n}\right)$ for some $n$. If $X$ has a proper characteristic generated core, then we have

(i) If $V=\Omega_{1}\left(Z\left(O_{2}(X)\right)\right)$, then $[V, X] / C_{[V, X]}(X)$ is the natural (i.e., the standard) module for $X / \mathrm{O}_{2}(X)$;

(ii) If $\bar{X}=X / V$, then $\bar{X}=O_{2}(\bar{X}) C_{\bar{X}}\left(O_{2}(\bar{X})\right)$; and

(iii) $A$ Sylow 2-subgroup of $X$ has class 2. 
Note that (i) and (ii) imply that $X$ has exactly one chief factor within $O_{2}(X)$ on which $X$ acts nontrivially (namely, $\left.[V, X] / C_{[V, X]}(X)\right)$. $(A / B$ is a chief factor of $X$ if $A$ and $B$ are normal subgroups of $X$ with $B<A$ such that $A / B$ is a minimal normal subgroup of $X / B$. We say that $A / B$ is within the subgroup $Y$ of $X$ if $A \leqslant Y$.) All the other chief factors of $X$ within $O_{2}(X)$ have order 2 .

Recently Glauberman has obtained an important refinement of Theorem 6 [226]. It is based on certain methods first introduced by Baumann and also utilizes some of Niles' pushing up techniques.

THEOREM 7. Let $X$ be a group with $F^{*}(X)=O_{2}(X)$ and $X / O_{2}(X) \cong L_{2}\left(2^{n}\right)$ for some $n$ and let $T \in S_{y} l_{2}(X)$. If $X=C(X ; T)$, then there exist preassigned characteristic subgroups $J^{*}(T)$ of $C_{T}\left(\Omega_{1}(Z(J(T)))\right.$ ) (in particular, $J^{*}(T)$ char $T)$, and $Z_{1}(T)$ of $T$ with $Z_{1}(T) \leqslant \Omega_{1}(Z(T))$ such that

$$
X=\left\langle C_{X}\left(Z_{1}(T)\right), N_{X}\left(J^{*}(T)\right)\right\rangle .
$$

In other words, the characteristic generated core of $X$ is determined in this case by the normalizers of just two characteristic subgroups of $T$. (Clearly then $Z_{1}(T)$ or $J^{*}(T)$ is normal in $X$ in this case.)

The subgroup $J^{*}(T)$ is very complicated. Its definition is similar in spirit to that of $\hat{J}(T)$ in Definition 10.11, but even more intricate.

All results of the above type are difficult to establish and involve very delicate commutator calculations within the lower central series of $T$, the aim of which is to produce a characteristic subgroup of $T$ which is normal in $X$. It turns out to be possible to accomplish this except when $O_{2}(X)$ has a very restricted structure.

For the sake of clarity, let us describe $N$ in Theorem 6 when $n \geqslant 2$ (here $N$ is normal in $X$ and minimal subject to $X=N C(X ; T)$ ). Since $T \leqslant C(X ; T)$ $<X$ and $X / O_{2}(X)$ is simple, $N$ must cover $X / O_{2}(X)$, whence $X=O_{2}(X) N$. The minimality of $N$ implies now that $N$ is perfect. Using (ii), it follows easily from this that $\bar{N}$ centralizes $\mathrm{O}_{2}(\bar{X})$ and hence centralizes $\mathrm{O}_{2}(\bar{N})\left(<\mathrm{O}_{2}(\bar{X})\right)$. Thus $O_{2}(\bar{N}) \leqslant Z(\bar{N})$ and as $\bar{N}$ is perfect, we conclude that $\bar{N}$ is quasisimple. By the results of Schur [164], either $\bar{N} \cong L_{2}\left(2^{n}\right)$ is simple or $n=2$ and $\vec{N} \cong S L(2,5)$. Thus $N V / V \cong L_{2}\left(2^{n}\right)$ or $S L(2,5)$. Applying the same argument again, we deduce that $N /[N, V] \cong L_{2}\left(2^{n}\right)$ or $S L(2,5)$. Since $N$ covers $X / O_{2}(X),[V, X]=[V, N](=[N, V])$ and by (i), $[N, V] / C_{[N, V]}(N)$ is a natural module. Since $N$ is perfect, it also follows that $N$ acts indecomposably on $[N, V]$. This gives a very precise picture of $N$. A similar description of $N$ exists in the case $n=1$.

Goldschmidt has recently extended the Baumann-Niles theorem to the case that $X / O_{2}(X) \cong A_{n}$ or $\Sigma_{n}, n$ odd [81]. The conclusions are identical this time with $[X, V] / C_{[X, V]}(X)$ a natural module for $X / O_{2}(X) \cong A_{n}$.

To treat the general local situation, Aschbacher has introduced the following terminology [12].

Definition 8. A group $X$ is said to be short provided $X=O^{2}(X)$ (i.e., $X$ has no normal subgroups of index 2$), F^{*}(X)=O_{2}(X), U(X)=\left[X, O_{2}(X)\right] \leqslant$ $\Omega_{1}\left(Z\left(O_{2}(X)\right)\right)$, and $X / O_{2}(X)$ is either quasisimple or of prime order. (The second alternative is included to allow for $A_{3} \cong Z_{3}$.) 
A short group $X$ is said to be a block if $X / O_{2}(X) \cong L_{2}\left(2^{n}\right), n \geqslant 1$ or $A_{n}, n$ odd, and $U(X) / C_{U(X)}(X)$ is a natural $X / O_{2}(X)$-module.

Finally $X$ is said to be a block of the group $Y$ if $X$ is a block and $X$ is subnormal in $Y$.

Thus a block is always a block of itself. To avoid possible confusion, we emphasize that the term block here has no connection with Brauer's character theoretic notions of blocks.

Aschbacher proves the following fundamental result [12].

THEOREM 9. Let $X$ be a group with $F^{*}(X)=O_{2}(X)$ such that $X$ has a proper characteristic generated core and let $T \in S y l_{2}(X)$. Then $X$ contains a normal subgroup $N$ with the following properties:

(i) $X=N C(X ; T)$;

(ii) $N=N_{1} N_{2} \cdots N_{r}$, where each $N_{i}$ is a block of $X, 1 \leqslant i \leqslant r$; and

(iii) $N_{i}$ centralizes $N_{j}$ for all $i, j, i \neq j, 1 \leqslant i, j \leqslant r$.

To establish the theorem, Aschbacher invokes a slightly more general form of the stated Theorem 11.7 to conclude that $N$ is a product of subgroups $N_{i}$ with $N_{i} / O_{2}\left(N_{i}\right) \cong L_{2}\left(2^{n_{i}}\right)$ or $A_{n_{i}}, n_{i}$ odd, $1 \leqslant i \leqslant r$. In effect, this reduces the proof (with a little more arguing) to the Baumann-Niles and Goldschmidt theorems. (Actually Aschbacher's paper treats the alternating case, as it was written prior to Goldschmidt's result.)

Since Theorem 11.7 depends on Timmesfeld's root involution theorem, so also does the preceding result. Thus we have one further indication of the breadth of applicability of the entire Fischer method to local group theory. (If one assumes that $X$ is a $K$-group, which is all that is needed for the applications, one could undoubtedly avoid the root involution theorem here; but then the elegance of the result would certainly be diminished.)

With the aid of Glauberman's Theorem 7, one can obtain the following refinement of Theorem 9 .

THeOReM 10. Let $X$ be a group with $F^{*}(X)=O_{2}(X)$ such that $X$ has a proper characteristic generated core and let $T \in \operatorname{Syl}_{2}(X)$. Then $X$ contains $a$ normal subgroup $N$ with the following properties:

(i) $X=N\left\langle C_{X}\left(\Omega_{1}(Z(T))\right), N_{X}\left(J^{*}(T)\right)\right\rangle$;

(ii) $N=N_{1} N_{2} \cdots N_{r}$, where each $N_{i}$ is a block of $X, 1 \leqslant i \leqslant r$; and

(iii) $N_{i}$ centralizes $N_{j}$, for all $i, j, i \neq j, 1 \leqslant i, j \leqslant r$.

Aschbacher has obtained an important variation of these results in a case in which $T$ is not a Sylow 2-subgroup of $X$ (but in which $X$ is a $K$-group) [14]. To motivate Aschbacher's result, return to the situation with $M$ and $X$ at the beginning of the section. In investigating the relationship between these two subgroups of the group $G$, the group $Y=X \cap M$ will certainly be important. In some circumstances, one can show that $N_{G}(R) \leqslant X$ for every $1 \neq R$ char $O_{2}(Y)$. It follows for any such $R$ that $N_{M}(R) \leqslant X \cap M=Y$. This turns out to be a powerful restriction on the group $M$ as the following theorem of Aschbacher clearly illustrates [14]. Here we have replaced $M$ by $X$ and put $T=O_{2}(Y)$ to conform to the notation of the preceding theorems. Furthermore, to state it, we broaden the definition of $C(X ; T)$ to cover arbitrary 
2-subgroups of a group $X$. Thus for any 2-subgroup $T$ of the group $X$, we set

$$
\left.C(X ; T)=\left\langle N_{X}(R)\right| 1 \neq R \text { char } T\right\rangle .
$$

THEOREM 11. Let $X$ be a $K$-group with $F^{*}(X)=O_{2}(X)$. Let $T$ be a 2-subgroup of $X$, set $Y=N_{X}(T)$, and assume that the following two conditions hold:

(a) $T=O_{2}(Y)$;

(b) $Y=C(X ; T)$.

Then one of the following holds:

(i) $Y=X$ and $T=O_{2}(X)$;

(ii) $X$ contains a $T$-invariant block $L$ with $L T / O_{2}(L T) \cong L_{2}\left(2^{n}\right)$ for some $n \geqslant 1$; or

(iii) If $\bar{X}=X / O_{2}(X)$, then $L(\bar{X})$ has a T-invariant component $\bar{L}$ such that $\bar{L} / Z(\bar{L})$ is either of Lie type of characteristic 2 or an alternating group. Furthermore, $(\bar{L} \cap \bar{Y}) \bar{T} / \bar{T}$ contains a subnormal subgroup which is either of Lie type of characteristic 2 and order divisible by 3 or is an alternating group.

Aschbacher's results are actually more precise. First of all, he argues that $T$ leaves invariant each component of $L(\bar{X})$. Furthermore, by hypothesis (a) and (b), $O_{2}(X) \leqslant T$. If $\bar{T}$ does not centralize $O(\bar{X})$, then as $\bar{T} \in$ $\mathrm{Syl}_{2}(O(\bar{X}) \bar{T})$, we conclude with the aid of Theorem 11.6 (applied to the preimage of $O(\bar{X}) \bar{T}$ in $X$ ) that $X$ contains a $T$-invariant block $L$ with $L T / O_{2}(L T) \cong L_{2}(2)$ and $O(\bar{L}) \leqslant O(\bar{X})$. In particular, (ii) holds. Thus we can assume that $\bar{T}$ centralizes $O(\bar{X})$, whence $\bar{T}$ does not centralize $\bar{L}$ for some component $\bar{L}$ of $L(\bar{X})$ (otherwise it follows that $T=O_{2}(X)$, whence $Y=X$ and (i) holds).

Let $X_{0}$ be the preimage of $\bar{L} \bar{T}$ in $X$. Then $T \neq O_{2}\left(X_{0}\right)$ (otherwise $\bar{T}$ would centralize $\bar{L})$. Since $T=O_{2}(Y)$, it follows that $X_{0} \nless Y$. Hence $C\left(X_{0} ; T\right)=Y$ $\cap X_{0}<X_{0}$. In particular, the $(Z, J)$-factorization fails for $X_{0}$ (relative to $T$ ). Since $X$ is a $K$-group, Aschbacher's Theorem 11.3 implies now that either $\bar{L} \in \operatorname{Chev}(2)$ or $\bar{L} \cong A_{n}$ for some $n$.

If $\bar{T} \cap \bar{L} \in \operatorname{Syl}_{2}(\bar{L})$, then $T \in \operatorname{Syl}_{2}\left(X_{0}\right)$. Since $C\left(X_{0} ; T\right)<X_{0}$, the Baumann-Niles-Goldschmidt theorems yield that either $X_{0}$ or $O^{2}\left(X_{0}\right)$ is a block. Aschbacher argues in the present situation that only blocks of type $L_{2}\left(2^{n}\right)$ can occur.

In the contrary case, Aschbacher analyzes the Chev(2) and alternating group subcases separately. In the alternating case, he invokes the very detailed description of the modules arising from failure of $(Z, J)$-factorization which he had earlier established (cf. Proposition 11.4). In particular, (iii) follows in this case.

On the other hand, if $\bar{L} \in \operatorname{Chev}(2)$, he sets $\tilde{X}_{0}=\bar{X}_{0} / O_{2}\left(\bar{X}_{0}\right)=\tilde{L} \tilde{T}$ and distinguishes two further subcases according as $\tilde{T}$ induces inner automorphisms on $\tilde{L}$ or not. In the first case, $\tilde{X}_{0}=\tilde{L}$ and setting $\bar{Y}_{0}=\bar{Y} \cap \bar{L}$, he argues that $\tilde{Y}_{0}$ is a parabolic subgroup of $\tilde{L}$ with $\tilde{T}=O_{2}\left(\tilde{Y}_{0}\right)$. Since $\bar{T} \cap \bar{L} \notin$ $\operatorname{Syl}_{2}(\bar{L}), \tilde{T} \notin S y l_{2}(\tilde{L})$, so $\tilde{L}$ has Lie rank at least 2 . Also, $\tilde{L} ¥{ }^{2} F_{4}\left(2^{n}\right)$ because of the failure of factorization, so 3 divides $\left|\tilde{Y}_{0}\right|$, inasmuch as the groups ${ }^{2} F_{4}\left(2^{n}\right)$ are the only groups of Lie type of characteristic 2 and Lie rank at least 2 having a parabolic of order prime to 3 . Again (iii) follows.

Finally if $\tilde{T}$ does not induce inner automorphisms on $\tilde{L}$, Aschbacher argues 
that $|\tilde{T}|=2$ and that $\bar{L} \cong O_{n}{ }^{ \pm}(2)$ with $\bar{T}$ inducing a transvection on $[V, \bar{L}]$, where $V=\Omega_{1}\left(Z\left(O_{2}(X)\right)\right)$.

13. General properties of $K$-groups. As we have repeatedly stressed, local analysis requires for its success specific properties of the $K$-groups involved in the simple group under investigation. Perhaps, when the finite simple groups have been completely determined, at which time all finite groups will be $K$-groups, the already established general properties of simple $K$-groups will provide a basis for a "theory of finite groups", analogous to the beautiful theory of solvable groups which has evolved since the original work of P. Hall in the 1920s and 1930s [104] $[107]$.

The standard procedure for establishing a general property of $K$-groups $X$ is to reduce the problem to a question about suitable simple (and quasisimple) sections of $\boldsymbol{X}$ (as, for example, in treating global balance or failure of factorization in 2-constrained groups). Thus our attention is soon focused on specific questions about the known simple groups. The major properties of simple $K$-groups which are needed for local analysis all seem to fall within (at least) one of the following categories:
A. Automorphisms.
B. Schur multipliers.
C. Centralizers of elements of prime order.
D. Balance.
E. Generation.
F. Subgroup structure.
G. Fusion.
H. Signalizers.

I. Representations over fields of prime order.

In this section I shall describe some of the principal results which have been obtained within each category. Some of these are easily proved, others are quite difficult. I shall not attempt to discuss any of their proofs, but shall limit myself to a few general comments.

In dealing with the groups of Lie type, the general Chevalley-Steinberg theory is of fundamental importance. In particular, it often allows one to make calculations which apply simultaneously to all groups of Lie type. The same remark applies to the alternating groups and their standard presentation. On the other hand, one must often analyze individual families (and even individual groups) separately, especially regarding properties which involve exceptions to the general case. Finally one must usually treat the sporadic groups one at a time, calculating or arguing on the basis of their precise definitions.

A. Automorphisms. If $X$ is a group of Lie type defined over $G F\left(p^{m}\right)$, every element of $\operatorname{Aut}(X)$ is known to be a product of an inner, a "diagonal", a "field", and a "graph" automorphism [186].

For example, diagonal matrices in $G L\left(n, p^{m}\right)$ of determinant unequal to 1 determine by conjugation diagonal automorphisms of $S L\left(n, p^{m}\right)$. Field automorphisms arise in a natural way from elements $\sigma$ of the Galois group of the underlying field $G F\left(p^{m}\right)$. Thus for $A=\left(a_{i j}\right) \in S L\left(n, p^{m}\right), a_{i j} \in G F\left(p^{m}\right)$, $1 \leqslant i, j \leqslant n$, the map 


$$
A=\left(a_{i j}\right) \mapsto A^{\sigma}=\left(a_{i j}^{o}\right)
$$

is an automorphism of $S L\left(n, p^{m}\right)$.

Likewise graph automorphisms are induced from symmetries of the Dynkin diagram of the associated Lie algebra of $X$. Thus the diagram associated with $S L\left(n, p^{m}\right)$

$$
\text { O-O-0 - . O - O }
$$

with $n-1$ nodes has a reflection of order 2 , which induces an automorphism of $S L\left(n, p^{m}\right)$ (in this case, taking the usual generators of $S L\left(n, p^{m}\right)$, the graph automorphism is the transpose-inverse map).

Diagonal automorphisms determine an abelian group of automorphisms (in fact, cyclic except for $D_{2 n}(q), n$ even, and $q$ odd), while field automorphisms always determine cyclic groups. On the other hand, graph automorphisms determine a group of order 1 or 2 , except for $D_{4}$, in which case they determine a group isomorphic to $\Sigma_{3}$. Moreover, there is a general formula for the order of the diagonal group for each family of groups of Lie type. Also the group of diagonal automorphisms is normal in $\operatorname{Aut}(X) / X$ and field automorphisms commute with graph automorphisms. Summarizing, we have

THEOREM 1. If $X$ is a group of Lie type, then $A=A u t(X) / X$ is a solvable group with normal subgroups $D$ and $D F$, where $D$ is abelian and $F$ is cyclic; and, moreover, $A / D F \cong 1, Z_{2}$, or $\Sigma_{3}$.

In the case of the alternating groups, one has

Theorem 2. (i) If $X=A_{n}, n \neq 6, n \geqslant 3$, then $A u t(X) \cong \Sigma_{n}$; and

(ii) If $X=A_{6}\left(\cong L_{2}(9)\right)$, then $\operatorname{Aut}(X) \cong \operatorname{Aut}\left(L_{2}(9)\right)\left(L_{2}(9)\right.$ has index 4 in $\left.\operatorname{Aut}\left(L_{2}(9)\right)\right)$.

The automorphism group of almost all of the sporadic group has been computed (in most cases by the individual who first determined its internal structure). In the course of their work on standard components, Aschbacher and Seitz [16] have established the following result (except for Janko's group $J_{4}$, for which the desired assertion can be easily checked).

THEOREM 3. If $X$ is a known sporadic group, then

$$
|\operatorname{Aut}(X) / X| \leqslant 2 \text {. }
$$

Combining these results, one has

THEOREM 4. For any known simple group $X, \operatorname{Aut}(X) / X$ is solvable.

Schreier conjectured that every simple group has a solvable outer automorphism group. In view of Theorem 4, the conjecture will follow as a corollary of the classification of all simple groups (at least if our present list is complete).

B. SCHUR MULTIPLIERS. The Schur multipliers of every known simple group apart from $F_{2}$ has been computed. The general answer for the classical groups has been known for a long time, but there are exceptions for certain groups over fields with few elements. Steinberg has given a systematic treatment for the groups of Lie type, apart from these exceptional cases and the Ree and 
Suzuki groups [185]. The Ree groups of characteristic 3 and the Suzuki groups were handled by Alperin and me [5], and the Ree groups of characteristic 2 (apart from the Tits group $\left.{ }^{2} F_{4}(2)^{\prime}\right)$ by $\mathrm{H}$. Ward [216]. Griess computed the Schur multipliers for the exceptional cases of Lie type and for many of the sporadic groups [98], [99]. Burgoyne, Fong, Janko, McKay, Norton, Thompson, Wales, and others have each treated one or more of the remaining sporadic groups [36], [146]. The multipliers of the alternating groups were determined by Schur [165].

THEOREM 5. If $X$ is a simple group of Lie type, then one of the following holds:

(i) $X$ has a cyclic Schur multiplier;

(ii) $X=P \Omega^{+}(4 n, q)$, $q$ odd, and $X$ has $Z_{2} \times Z_{2}$ as Schur multiplier; or

(iii) $X$ is included among the groups in the table below.

The exact order of the Schur multiplier has been determined for each group in (i). The following table lists those groups of Lie type whose Schur multipliers differ from those of the general member of the corresponding families of groups.

$\begin{array}{llll}\text { Chevalley group } & \text { multiplier } & \text { Twisted group } & \text { multiplier } \\ A_{1}(4) \cong A_{5} \cong A_{1}(5) & Z_{2} & { }^{2} A_{3}(2) \cong C_{2}(3) & Z_{2} \\ A_{1}(9) \cong A_{6} & Z_{2} \times Z_{3} & { }^{2} A_{3}(3) & Z_{4} \times Z_{3} \times Z_{3} \\ A_{2}(2) \cong A_{1}(7) & Z_{2} & { }^{2} A_{5}(2) & Z_{2} \times Z_{2} \times Z_{3} \\ A_{2}(4) & Z_{4} \times Z_{4} \times Z_{3}{ }^{2} B_{2}(8) \cong S z(8) & Z_{2} \times Z_{2} \\ A_{3}(2) \cong A_{8} & Z_{2} & { }^{2} E_{6}(2) & Z_{2} \times Z_{2} \times Z_{3} \\ B_{2}(2) \cong \Sigma_{6} & Z_{2} & & \\ B_{3}(2) \cong C_{3}(2) & Z_{2} & \\ B_{3}(3) & Z_{2} \times Z_{3} & & \\ D_{4}(2) & Z_{2} \times Z_{2} & & \\ F_{4}(2) & Z_{2} & \\ G_{2}(3) & Z_{3} & \\ G_{2}(4) & Z_{2} & \end{array}$

The next table lists the Schur multipliers for the alternating groups.

$$
\begin{array}{ll}
A_{n}, n \neq 6 \text { or } 7, n \geqslant 5, & Z_{2} \\
A_{6} & Z_{2} \times Z_{3} \\
A_{7} & Z_{2} \times Z_{3}
\end{array}
$$

Finally we list those sporadic groups having nontrivial (or undetermined) Schur multipliers. (The existence of the monster $F_{1}$ would suffice to prove that $F_{2}$ has $Z_{2}$ as its multiplier. However, at present there is no independent proof of this result.) 


$\begin{array}{llll}M_{12} & Z_{2} & .1 & Z_{2} \\ M_{22} & Z_{2} \times Z_{3} & M(22) & Z_{2} \times Z_{3} \\ J_{2} & Z_{2} & M(24)^{\prime} & Z_{3} \\ J_{3} & Z_{3} & F_{2} & Z_{2}(?) \\ O N & Z_{3} & & \\ H S & Z_{2} & & \\ M c & Z_{3} & & \\ S u z & Z_{2} \times Z_{3} & & \\ R u & Z_{2} & & \end{array}$

C. Centralizers of elements of PRime $p$. To keep the discussion within bounds, we shall limit our statements to centralizers of involutions. Analogous results hold in many cases for centralizers of elements of odd prime order $p$; in particular, for groups $X$ of Lie type over $G F(q)$ when either $p$ divides the order of a Cartan subgroup of $X$ or $p=3$ and $q=2$ (two cases especially important for the applications). Note that we have already made some comments about centralizers of involutions in §I.5; and again we refer the reader to [37], [38], [128], [162].

We let $X$ be a group such that $Y=F^{*}(X)$ is a simple $K$-group, we let $t \in G(X)$, and we set $C=C_{X}(t)$.

THEOREM 6. If $t \notin Y$, then one of the following holds:

(i) $C$ is solvable;

(ii) $F^{*}(C)=L(C)$ is quasisimple;

(iii) $Y \in C h e v(p)$ for some $p$ and $t$ is a diagonal automorphism;

(iv) $Y \cong L_{4}(q)$ or $U_{4}(q), q$ odd, $t$ is the transpose-inverse automorphism (or $t$ is an element in its coset) and $L(C) \cong L_{2}(q) \times L_{2}(q)$; or

(v) $Y \cong A_{n}$ and $t$ is not a transposition.

Furthermore, it also follows that $L(C)$ is a $K$-group and either $C / L(C)$ is solvable or $Y \cong A_{n}$.

THEOREM 7. If $t \in Y$, then one of the following holds:

(i) $L(C)$ is trivial or quasisimple;

(ii) $Y \in C h e v(p), p$ odd, and $L(C)$ has two components; or

(iii) $Y \cong P \Omega^{ \pm}(q), q$ odd, and $L(C)$ has three or four components.

Furthermore, if $L(C)$ is nontrivial, then either $C / L(C)$ is solvable or $Y \cong A_{n}$.

Again the possibilities for $L(C)$ are known for each choice of $Y$ and $t$. In particular, we have

THEOREM 8. If $t \in Y$ and $L(C)$ is trivial, then one of the following holds:

(i) $C$ is solvable;

(ii) $F^{*}(C)=O_{2}(C)$; or

(iii) $Y \cong A_{n}, n \equiv 3(\bmod 4)$, and $F^{*}(C)=O_{2}(C) \times O_{3}(C)$ with $O_{3}(C) \cong$ $Z_{3}$.

Combining these last three results (together with the known structure of $C$ in cases (iii) and (v) of Theorem 6), we obtain as a consequence the 
fundamental so-called " $B$-property" of the layers of the centralizers of involutions in the known simple groups.

THEOREM 9. For any choice of $X$ and $t$, we have

$$
L_{2^{\prime}}(C)=L(C) \text {. }
$$

A large portion of Chapter VI will deal with the $B$-property and the related " $B$-conjecture" for arbitrary finite groups. As we shall see, this whole subject is closely connected to the question of groups containing involutions whose centralizers have nontrivial cores. Since this is the same as saying that the group $Y$ is not locally balanced, Proposition 3.6 yields the following result for $K$-groups $X$ with $Y=F^{*}(X)$ simple.

THEOREM 10. If $O\left(C_{X}(t)\right) \neq 1$, then one of the following holds:

(i) $Y \in C h e v(p)$ for some odd prime $p$;

(ii) $Y \cong A_{n}, n$ odd, $n \geqslant 7$; or

(iii) $Y \cong L_{3}(4)$ or He and $t \notin Y$.

If $t$ is the "unitary" automorphism of $Y=L_{3}(4)$, then $C_{Y}(t) \cong U_{3}(2)$, which is solvable and, in fact, a split extension of $Z_{3} \times Z_{3}$ by $Q_{8}$, so $O(C)=$ $O\left(C_{Y}(t)\right) \cong Z_{3} \times Z_{3}$. Likewise $Y=H e$ admits an outer automorphism $t$ of order 2 such that $C_{Y}(t)$ is a 3 -fold cover of $\Sigma_{7}$, so $O(C)=O\left(C_{Y}(t)\right) \cong Z_{3}$.

Thompson has reduced a major case of the $B$-conjecture to the verification of four specific properties of the centralizers of involutions in groups of Lie type [201]. Burgoyne has since completed the verification of each of the desired properties [34]. To indicate their nature, we list the principal two here.

We consider a group $X$ in which $Y=F^{*}(X) \in \operatorname{Chev}(p), p$ odd, with $Y \approx L_{2}\left(p^{n}\right)$; and if $p=3$, also $Y \approx{ }^{2} G_{2}\left(3^{n}\right)^{*}$. (The excluded groups are the only members of $\operatorname{Chev}(p)$ which do not contain subgroups isomorphic to $S L(2, p)$.) Note that by definition of $\operatorname{Chev}(p), Y$ is quasisimple, but not necessarily simple. Again we let $t \in \mathscr{G}(X)$ and set $C=C_{X}(t)$.

THEOREM 11. Assume $Y$ is simple and $O(C) \neq 1$. Then there exists an involution $u \in C$ and a subnormal subgroup $L$ of $C_{X}(u)$ with the following properties:

(i) $L \cong S L(2, q)$ for some odd $q$ and $\langle u\rangle=Z(L)$; and

(ii) $\langle O(C), t\rangle$ normalizes $L$ and $C_{\langle O(C), t\rangle}(L)=1$.

THEOREM 12. Assume $L(C)$ has a component $L$ with the following properties:

(a) $L \cong S L(2, q)$, for some odd $q$;

(b) $L$ is normal in $C$; and

(c) $Z(L) \leqslant Z(X)(\leqslant Z(Y))$.

Then $Y / Z(Y)$ is isomorphic to one of the following groups: $L_{4}(q), U_{4}(q)$, or $P \Omega^{ \pm}(n, q)$, where $q=p^{m}$ for some $m$.

Further properties of centralizers of involutions are needed for many of the results listed in subsections (D)-(I) of this section.

D. BALANCE. By now, the reader should have a pretty good idea of the general spirit of the theory of $K$-groups that is required for local analysis. Hence I shall be briefer in discussing the remaining types of properties. Proposition 3.6, Theorem 3.11, and Proposition 3.12 describe local $k$-balance 
for simple $K$-groups. This is the principal balance property needed for local analysis. However, as indicated in the discussion of Goldschmidt type signalizer functors in $\$ 3$, variations of local $k$-balance are also used in the applications.

E. Generation. We have remarked several times on the importance of generational statements for local analysis-in particular, for the construction of signalizer functors (Proposition 2.9). Generational questions also arise in the stage following this construction. For example, suppose $G$ is a simple group which is 2-balanced for the prime $p, A$ an elementary abelian $p$-subgroup of $G$ with $m_{p}(A)>4$, and the associated 2-balanced $A$-signalizer functor $\theta$ is complete. If we set $M=N_{G}(\theta(G ; A))$, it is immediate that

$$
\Gamma_{A, 3}(G)=\left\langle N_{G}(E) \mid E \leqslant A, m_{p}(E)>3\right\rangle<M .
$$

Assuming $\theta(G ; A) \neq 1$, the ultimate goal of the analysis is to prove that $M$ is strongly embedded in $G$. In particular, we must argue that $C_{a} \leqslant M$ for each $a \in A^{\#}$. We cannot expect to reach such a strong conclusion solely from (3); however, (3) does yield some partial information. Indeed, as $m_{p}(A) \geqslant 4$, it follows easily from Proposition 1.4.7 that

$$
O_{p^{\prime}}\left(C_{a}\right) \leqslant \Gamma_{A, 3}(G) \leqslant M .
$$

Hence if we set $\bar{C}_{q}=C_{a} / O_{p^{\prime}}\left(C_{a}\right)$, we can work with $\bar{C}_{a}$ and $\bar{A}$ inasmuch as $N_{C_{a}}(E)$ covers $N_{\bar{C}_{a}}(E)$ for every subgroup $E$ of $A$. The natural first question to ask is whether

$$
L\left(\bar{C}_{a}\right)<\Gamma_{\bar{A}, 3}\left(\bar{C}_{a}\right)=\left\langle N_{\bar{C}_{a}}(\bar{E}) \mid \bar{E} \leqslant \bar{A}, m_{p}(\bar{E}) \geqslant 3\right\rangle .
$$

If (5) holds, then $M$ will cover $L\left(\bar{C}_{a}\right)$ and it will follow that $L_{p^{\prime}}\left(C_{a}\right)<M$, which would be an important conclusion for any subsequent analysis.

One can easily reduce this problem to the corresponding generational question for $\bar{A}$-invariant quasisimple sections $\bar{X}$ of $L\left(\bar{C}_{a}\right)$ such that $\bar{X}$ is a homomorphic image of some component of $L\left(\bar{C}_{a}\right)$. Clearly for generational purposes, only the group $\tilde{A}=\bar{A} / C_{\bar{A}}(\bar{X})$ can present an obstruction to generation. If $|\tilde{A}| \leqslant p$, then $m_{p}\left(C_{\bar{A}}(\bar{X})\right) \geqslant 3$ and the desired generation holds trivially. Hence one can assume that $\tilde{A}$ is noncyclic. Also a suitable generational statement for $\tilde{X}=\bar{X} / Z(\bar{X})$ relative to $\tilde{A}$ implies one for $\bar{X}$ relative to $\bar{A}$. Since, in particular, $X$ will be a $K$-group, we are thus reduced to studying the faithful action of noncyclic elementary abelian p-groups on simple $K$ groups.

Seitz has obtained definitive results for odd $p$ for groups of Lie type of characteristic not $p$ [167]. The corresponding results for the sporadic groups have been computed by Lyons, using O'Nan's information concerning their local subgroups [156]. Generation always fails for the groups in $C h e v(p)$ and in the case of alternating groups, it depends strongly upon the choice of elementary abelian $p$-group. We limit ourselves to the following statement.

THEOREM 13. Let $X$ be a simple $K$-group and $A$ a noncyclic elementary abelian p-subgroup of $\operatorname{Aut}(X), p$ odd, and assume

$$
\left.\left\langle N_{X}(E)\right| E \leqslant A \text { and }|A: E| \leqslant p\right\rangle\langle X .
$$


Then one of the following holds:

(i) $X \in \operatorname{Chev}(p)$ or $X \cong A_{n}$;

(ii) $p=11, A \leqslant X$, and $X$ is of type $J_{4}$;

(iii) $p=5, A \leqslant X$, and $X \cong{ }^{2} F_{4}(2)^{\prime}, M c$, or $M(22)$;

(iv) $p=3, A \leqslant X, X \cong P s p(n, 2), U_{n}(2), P \Omega_{n}^{ \pm}(2), G_{2}(2)^{\prime}, F_{4}(2), L_{3^{k}}(4), F_{4}(4)$,

${ }^{2} E_{6}(2), E_{6}(4), E_{7}(2), E_{8}(2), M_{11}, J_{2}, J_{3}, J_{4}, O N ;$ or $H S$;

(v) $p=5, X \cong S z\left(2^{5}\right)$ or ${ }^{2} F_{4}\left(2^{5}\right)$ and $A X \cong \operatorname{Aut}\left(S z\left(2^{5}\right)\right)$ or $\operatorname{Aut}\left({ }^{2} F_{4}\left(2^{5}\right)\right)$;

(vi) $p=3, X \cong L_{2}\left(2^{3}\right)$, and $A X \cong A u t\left(L_{2}\left(2^{3}\right)\right)$;

(vii) $p=3, X \cong D_{4}(2)$ or $D_{4}(4)$, and $A X=\langle a\rangle X$, where $a \in A^{\#}$ induces the graph automorphism of $X$; or

(viii) $p=3, X \cong P s p\left(n, 2^{3}\right), U_{n}\left(2^{3}\right), P \Omega_{n}^{ \pm}\left(2^{3}\right), G_{2}\left(2^{3}\right), F_{4}\left(2^{3}\right), L_{3^{k}}\left(4^{3}\right), F_{4}\left(4^{3}\right)$,

${ }^{2} E_{6}\left(4^{3}\right), E_{6}\left(4^{3}\right), E_{7}\left(2^{3}\right)$, or $E_{8}\left(2^{3}\right)$ and $A X=\langle a\rangle X$, where $a \in A^{\#}$ induces $a$ field automorphism on $X$.

Using this result, one can easily compute all simple $K$-groups which possess a strongly $p$-embedded subgroup for some odd prime $p$.

THEOREM 14. Let $X$ be a group such that $Y=F^{*}(X)$ is a simple $K$-group. If $X$ has a strongly $p$-embedded subgroup for some odd prime $p$, then one of the following holds:

(i) $X$ has cyclic Sylow $p$-subgroups;

(ii) $X \cong L_{2}\left(p^{n}\right), U_{3}\left(p^{n}\right)$ or ${ }^{2} G_{2}\left(3^{n}\right)^{*}$ (with $p=3$ or $p \mid 3^{3 n}+1$ in the last case);

(iii) $p=5$ and $X \cong{ }^{2} F_{4}(2)^{\prime}, \operatorname{Aut}\left(S z\left(2^{5}\right)\right)$, $M c$, or $M(22)$; or

(iv) $p=3$ and $X \cong L_{3}(4), \operatorname{Aut}\left(L_{2}\left(2^{3}\right)\right)$, or $M_{11}$.

F. Subgroup STRUCtURe. Principally, but not exclusively, local analysis requires information about the local subgroup and Sylow subgroup structure of simple $K$-groups. The Borel-Tits theorem (Theorem I.5.18) shows that every maximal $p$-local in a group of $C h e v(p)$ is a maximal parabolic and conversely. Thus the structure of the parabolic subgroups of such a group $X$ is very important. On the other hand, if $q$ is prime distinct from $p$, it is difficult to formulate a general description of the $q$-local subgroups of $X$, but properties of these subgroups can be deduced from the general structure of $X$ as a $(B, N)$-pair, sometimes with much effort. A similar remark applies to the alternating and sporadic groups. In the latter case, this effort has already been made and essentially all local subgroups of the sporadic groups have been determined.

Concerning the Sylow $p$-subgroups $P$ of a simple $K$-group $X$, we are usually interested only in the rank of $P$ or perhaps the structure of $J(P)$; but sometimes, particularly when $p=2$, we need more detailed structural information. Carter and Fong [40] have given a complete description of the Sylow 2-subgroups of the classical groups over fields of odd characteristic $q$, from which any needed properties can be read off. The corresponding results for $p \neq 2$ or $q$ were obtained by Weir [217]. These descriptions by CarterFong and Weir are very similar to the known structures of the Sylow $p$-subgroups of $A_{n}$. The Sylow structures of most of the remaining simple $K$-groups have been determined by the combined work of a great many

${ }^{12}$ The Ree groups ${ }^{2} G_{2}\left(3^{n}\right)$ are known to have cyclic Sylow $p$-subgroups for odd $p \mid 3^{3 n}+1$; however, this result has not been proved for the groups ${ }^{2} G_{2}\left(3^{n}\right)^{*}$ of Ree type. 
authors, usually in the course of various classification theorems. In particular, a description of the Sylow $p$-subgroups of the groups in $\operatorname{Chev}(p)$ involve the explicit commutator formulas of the Steinberg relations.

G. FUSION. One needs a considerable amount of information about the conjugacy classes of elements of prime order in simple $K$-groups. One often wants a precisely described set of representatives for, say, the conjugacy classes of involutions. For example, if $X=A_{n}$, the involutions (12)(34), (12)(34)(56)(78), . . constitute such a set. On the other hand, if $X \in$ Chev $(p), p$ odd, one can obtain representatives for the classes of involutions contained in a Cartan subgroup from the action of the Weyl group. However, there may be additional conjugacy classes of involutions (these have been determined in each case). In [15], Aschbacher and Seitz have computed all conjugacy classes of involutions of $X$ in the case that $Y=F^{*}(X) \in \operatorname{Chev}(2)$ (and have also determined the centralizers in $Y$ of each such involution). Finally, with the exception of $F_{2}$, all conjugacy classes of the sporadic groups have been determined (often as a preliminary step to computing their character tables).

We consider two general questions about fusion. First, if $X$ is a simple $K$-group, does the analogue of Glauberman's $Z^{*}$-theorem hold in $X$ for odd primes $p$ ? In other words, if $P \in S y l_{p}(X)$ and $Z \leqslant P$ has order $p$, must $Z$ fuse in $X$ to some subgroup of $P-Z$ ? (For odd $p$, the natural extension is to subgroups of order $p$ rather than elements of order $p$.) As in the involution case, we say that $Z$ is isolated in $P$ with respect to $X$ if $Z$ does not fuse to a subgroup of $X-Z$

If $P$ is cyclic, then $Z=\Omega_{1}(P)$ is the unique subgroup of $P$ of order $p$, so certainly $Z$ is isolated in $P$ with respect to $X$. Hence the question is of interest only when $P$ is noncyclic. The following statement has been checked for many, but not all, families of simple $K$-groups.

(?) THEOREM 15. Let $X$ be a simple $K$-group with noncyclic Sylow $p$-subgroup $P, p$ odd. If $P$ contains a subgroup of order $p$ which is isolated in $P$ with respect to $X$, then either $X \cong{ }^{2} G_{2}\left(3^{n}\right)^{*}$ with $p \mid 3^{3 n}+1$ or else $m_{p}(X)=2$ and one of the following holds:

(i) $X \cong U_{3}(p)$;

(ii) $p=5$ and $X \cong M c$; or

(iii) $p=3$ and $X \cong G_{2}(q),{ }^{3} D_{4}(q), q \neq 3^{n}$, or $J_{2}$.

In the study of groups of component type, the notion of a "tightly embedded" subgroup, due to Aschbacher, plays a fundamental role.

Definition 16. A subgroup $H$ of even order of a group $X$ is said to be tightly embedded in $X$ provided $H \cap H^{x}$ has odd order for every $x \in X-$ $N_{X}(H)$.

This concept is a direct generalization of strong embedding, which requires $H \cap H^{x}$ to have odd order for every $x \in X-H$. (Thus if $H$ is tightly embedded in $X$ and $H=N_{X}(H)$, then $H$ is strongly embedded in $X$.)

Obviously any subgroup of $X$ of order 2 is tightly embedded in $X$. In a very important paper, Aschbacher has essentially characterized the groups of Lie type of odd characteristic by the property of possessing a tightly embedded subgroup with quaternion Sylow 2-subgroups [10]. A full discussion of Asch- 
bacher's theorem will be given in \$VI.6. Furthermore, Aschbacher and Seitz have studied simple groups $G$ which possess a tightly embedded subgroup of 2-rank at least 2 under suitable additional assumptions which arise naturally in the study of simple groups of component type and have determined the exact possibilities for $G$ under these conditions [9], [15], [16]. Their work will be described in $\$$ VI.11.

Ultimately their analysis reduces to the determination of those finite groups $X$ with $F^{*}(X)$ quasisimple which contain a tightly embedded subgroup $H$ of 2-rank at least 2 satisfying a number of side conditions. In particular, $H$ must have (noncyclic) elementary abelian Sylow 2-subgroups and a normal 2-complement. The additional conditions are more technical and we postpone a discussion of them until Chapter VI. For brevity, let us just say that $H$ is restricted if it satisfies all these various conditions. An example of such a restricted tightly embedded subgroup $H$ is the subgroup $\langle(12)(34),(13)(24)\rangle$ $\cong Z_{2} \times Z_{2}$ in $A_{n}$.

The following theorem lists a few of Aschbacher-Seitz's results on restricted tightly embedded subgroups.

THEOREM 17. Let $X$ be a group such that $Y=F^{*}(X)$ is a simple $K$-group which contains a restricted tightly embedded subgroup $H$. Then we have

(i) Either $Y \in C h e v(2), Y \cong A_{n}$ for some $n$, or $Y \cong M_{12}, M_{24}, J_{2}, \mathrm{He}$, Suz, $R u$, or .1.

(ii) If $Y \cong A_{n}, n \geqslant 10$, then in the natural representation of $A_{n}, H$ is conjugate to $\langle(12)(34),(13)(24)\rangle$; and

(iii) If $Y \in C h e v(2)$, then a Sylow 2-subgroup of $H$ induces inner automorphisms on $Y$.

The effect of (iii) is to reduce the analysis (when $Y \in$ Chev(2)) to the case that $X=Y$. Aschbacher-Seitz obtain rather complete information in this case concerning the possible structures of (restricted) tightly embedded subgroups.

H. Signalizers. Let $X$ be a simple $K$-group and $P$ a $p$-subgroup of $X$. One needs various properties of $P$-signalizers in $X$-i.e., the collection of $P$-invariant $p^{\prime}$-subgroups of $X$-primarily when $P$ is either elementary abelian or a Sylow $p$-subgroup of $X$. For $p=2$, two questions are of special interest: $q$ ?

(a) Does $P$ normalize a nontrivial $q$-subgroup of $X$ for various odd primes

(b) Are any two maximal $P$-invariant $q$-subgroups of $X$ conjugate by an element of $N_{X}(P)$ (or even by an element of $C_{X}(P)$ )?

For odd $p$, the primary questions of interest are:

(c) What is the 2-local $p$-rank $m_{2, p}(X)$ ?

(d) Is $m_{p}(X)>m_{2, p}(X)$ ?

(e) If $P$ lies in a 2-local subgroup of $X$ and $m_{p}(P)=e(X)$ (the maximum of $m_{2, q}(X)$, taken over all odd primes $\left.q\right)$, can one describe the embedding of $P$ in $X$ ?

Here are a few of the illustrative results.

THEOREM 18. Let $X$ be simple of Lie type defined over $G F(q)$. Then we have

(i) If $P \in S_{y} l_{p}(X)$ and $q=p^{n}$, then $P$ has trivial signalizers.

(ii) If $p$ is odd, $q=2^{n}$, and $m_{2, p}(X)=e(X)$, then $p$ divides $q^{2}-1$. 
Theorem 19. (i) If $X=A_{n}$ and $P \in S y l_{2}(X)$, then a maximal P-invariant subgroup of $X$ of odd order is either trivial or of order 3; and, in the later case, $n \equiv 3(\bmod 4)$.

(ii) If $X$ is a sporadic simple group and $m_{2, p}(X) \geqslant 3, p$ odd, then $m_{p}(X)>$ $m_{2, p}(X)$.

We should also like to mention an important signalizer result of Thompson for $p$-constrained groups, $p$ odd [202].

THEOREM 20. Let $X$ be a p-constrained group, $p$ odd, and $P$ a p-subgroup of $X$ with the property that $P$ contains every element of order $p$ in $C_{P}(X)$. Then every $P$-signalizer of $X$ lies in $O_{p}(X)$.

I. REPRESENTATIONS OVER FIELDS OF PRIME ORDER. We have discussed this topic in some detail in $\$ \S 5,10$, and 11 , which should give a clear indication of the kind of module properties one needs for local analysis.

In this section, we have tried to describe the "general" theory of simple $K$-groups. However, in some classification problems, particularly low rank problems, one also needs a "special" theory of simple $K$-groups. For example, in the classification of groups with dihedral, quasi-dihedral, or wreathed Sylow 2-subgroups, one needs a "dictionary" of specialized properties of the groups $L_{2}(q), L_{3}(q), U_{3}(q), q$ odd, and $A_{7}$ (in most cases related to one of (A)-(I) above).

One also needs a theory of arbitrary $K$-groups (both general and special). Moreover, it is not always a routine matter to pass from properties of the simple sections of a $K$-group $X$ to properties of $X$ itself. For example, Thompson's $N$-group paper begins with over fifty preliminary lemmas, dealing primarily with solvable groups, some quite general and others very specialized. Some are easy to prove, but others take considerable effort.

Typically the proof of any classification theorem begins with such a list of detailed preliminary lemmas which can be applied to the proper sections of a minimal counterexample $G$ to the theorem, some concerning simple sections, others dealing with arbitrary $K$-groups which may be involved in $G$. Certainly one of the important tasks for the future will be to develop a coherent, systematic treatment of the entire theory of $K$-groups. 


\section{APPENDIX}

\section{A program for classifying the finite simple groups}

Four lectures delivered at the University of Chicago, June, 1972.

Introduction. It was with considerable hesitation that I accepted Alperin's suggestion to present an overview of the problem of classifying simple groups. In the face of the seeming vastness of the problem, who can have confidence in the validity of his own personal vision. It is not that I am afraid of making some foolish suggestions-I'm perfectly willing to do that in casual, private conversations-but one feels a deeper responsibility for mathematical accuracy in a formal setting.

After all, our fifteen or so years of effort have essentially concentrated on two extremities of the problem: first, close to the very base-by conditions that tightly restrict the composition factors of the local subgroups; and second, close to the known simple groups-by conditions that specify some subgroup structure very precisely. How clear a picture can one expect to get from such limited perspectives?

Imagine that I were called on to predict the final shape of the odd order problem at just the moment that Feit, Hall, and Thompson had completed the special case in which centralizers of elements were nilpotent. Perhaps I would have managed some accurate remarks about local analysis and character theory, but all the marvelous techniques of the general paper were far beyond my vision-and the final generator-relations case of Chapter VI was at that time completely hidden.

Having made these cautionary remarks, perhaps I should begin by briefly describing the sources of my present picture. Certainly the study of groups of low 2-rank-a task on which I have been endlessly submerged-is hardly designed to reveal the contours of outer space. Nevertheless, all my initial understanding of "components" developed out of this work.

However, far more important for my overall view has been Thompson's classification of $N$-groups. And now his recent work on 3'-groups reinforces my belief that the major subdivisions of the $N$-group paper are not accidental.

The abelian Sylow 2-group problem has also been a major factor. Because of the existence of composition factors isomorphic to $L_{2}(q), q$ odd, within the local subgroups, this problem included important features not present in the $N$-group or $3^{\prime}$-group cases. After John Walter's original classification theorem, he and I made a considerable effort to understand and generalize the intrinsic features of this problem. Out of this work, the whole signalizer functor method developed-the signalizer functor theorem itself, the theory of $k$-balanced groups, and a deeper understanding of components and nonconstrained local subgroups.

Although personally I have had only superficial contact with the sporadic groups, taken together they have had their effect on my picture. In fact, an 
analysis of their common internal features suggests to me a major problem area of future research.

Finally, all the characterization theorems of the known simple groups by centralizers of involutions has led me to believe in the following metaprinciple: If in a given classification problem, one is able to force the centralizer of an involution of $G$ to have some essentially precise form, then methods exist for determining the corresponding possibilities for $G$. The effect of this principle is to transform the total classification problem into the more functional problem of developing methods to pin down the structures of the centralizers of involutions in simple groups.

There you have the background for my present picture. Despite the reservations I expressed before of its inherent limitations, I do feel strongly that it represents as reasonably accurate a roadmap as it is possible to put forth at the present time. And I hope that presenting it here will help to channel some of your future research in what I believe to be fruitful directions.

This has been an important consideration for me in agreeing to speak on this subject, for I cannot help wondering whether all the classification theorems filling up the journals will really be needed for the ultimate classification of simple groups. Obviously, one cannot be certain, since any picture one has is subject to change without notice. But at least a global roadmap can provide you with some measure of the significance of a particular problem.

Let me illustrate. In the general classification of simple groups of 2-ranks 2, 3 and 4, it was necessary to first establish characterization theorems for the known simple groups by their Sylow 2-subgroups. This suggested a general problem area-and papers are now beginning to flow in this direction-each representing hard work and good mathematics. However, from the point of view of the program I shall outline, these results will, in general, not be significant for the classification of simple groups. The point is that for higher 2-rank, it is likely that one will require only characterization theorems by the centralizers of involutions.

On the other hand, the program will suggest another large area of research, which utilizes much the same techniques as the preceding: Namely, the characterization of the groups of Lie type of characteristic 2 by the structure of the centralizers of elements of order 3. Of course, everything I have to say may in the end turn out to be nonsense, but if it does represent the clearest picture we now have, one must ask himself why he should choose to work in the first problem area rather than the second.

Preliminary definitions and results. It will be best to start with some general terminology which will be needed in describing the specific parts of the program.

Definition. A quasisimple group is a perfect central extension of a simple group. A semisimple group is any central product of quasisimple groups. Its quasisimple factors are uniquely determined and are called the components of the semisimple group. For convenience, the trivial group of order 1 is also considered to be a semisimple group. 
Any group $H$ possesses a unique maximal normal semisimple subgroup, which we denote by $L(H)$.

Proposition. For any prime $p, H$ is p-constrained if and only if $L\left(H / O_{p^{\prime}}(H)\right)=1$.

Any group $H$ possesses a unique subgroup which is minimal subject to being normal in $H$ and covering $L\left(H / O_{p^{\prime}}(H)\right)$. We denote it by $L_{p}(H)$ and call it the p-layer of $H$.

Proposition. The following three statements are equivalent:

(i) $L_{p^{\prime}}(H) \subseteq L(H) ;^{13}$

(ii) $L_{p}(H)$ is semisimple;

(iii) $L_{p^{\prime}}(H)$ centralizes $O_{p^{\prime}}(H)$.

Definition. For any prime $p, \mathscr{E}_{k, p}(H)$ will denote the set of elementary abelian $p$-subgroups of $H$ of rank $k$. For brevity, we write $\mathcal{E}_{k}(H)$ for $\mathcal{E}_{k, 2}(H)$. If $T \in \mathcal{E}_{k, p}(H)$, we set

$$
\Delta_{H}(T)=\bigcap_{t \in T^{*}} O_{p^{\prime}}\left(C_{H}(t)\right) .
$$

A quasisimple group $L$ is said to be $k$-balanced for $p$ if for any subgroup $H$ of $\operatorname{Aut}(L)$ containing $\operatorname{Inn}(L)$ and any $T$ in $\mathcal{E}_{k, p}(H)$, we have

$$
\Delta_{H}(T)=1 \text {, }
$$

For brevity, we say $H$ is $k$-balanced if $p=2$ and we say $H$ is balanced for $p$ if $H$ is 1 -balanced for $p$.

Among the known simple groups, it appears to be the case that apart from the families $L_{2}(q), q$ odd, and $A_{n}$, they are all 2-balanced. The groups $L_{2}(q), q$ odd, are 3-balanced. However, for any $k$, the groups $A_{n}$ with $n \equiv 3\left(\bmod 2^{k}\right)$ are not $k$-balanced.

Definition. The $p$-rank of $H$ is the maximum rank of an elementary abelian $p$-subgroup of $H$. We denote it by $m_{p}(H)$ and set $m(H)=m_{2}(H)$. Likewise the sectional $p$-rank of $H$ is the maximum of $m_{p}(X), X$ ranging over all sections of $H$. We denote it by $r_{p}(H)$ and set $r(H)=r_{2}(H)$.

For example, if $\mathrm{SCN}_{3}(2)$ is empty in $\mathrm{H}$, it is known by a result of MacWilliams that $r(H) \leqslant 4$.

Definition. For any prime $p$, if $P$ is a Sylow $p$-subgroup of $H$, we set

$$
\left.\Gamma_{P, k}(H)=\left\langle N_{H}(Q) \mid Q \subseteq P, m_{p}(Q)\right\rangle k\right\rangle .
$$

We call $\Gamma_{P, k}(H)$ the $k$-generated $p$-core of $H$. (As usual, we suppress $p$ in the case $p=2$.) Clearly the $k$-generated $p$-core of $H$ is determined only up to conjugacy by the choice of $P$.

We say that $H$ is $k$-generated for $p$ if

$$
H=\Gamma_{P, k}(H)
$$

for some (and hence all) Sylow $p$-subgroup $P$ of $H$.

We say that $H$ possesses a strongly p-embedded subgroup if $H$ has a

\footnotetext{
${ }^{13}$ We have kept the notation of the original manuscript of these lectures. In particular, $\subseteq$ denotes subgroup containment, rather than $<$, as in the body of the article. There are a few other minor notational differences.
} 
nontrivial proper 1-generated $p$-core (i.e. $1 \subset \Gamma_{P, 1}(H) \subset H$ ). When $p=2$, we again drop the $p$ and note that our definition is equivalent to the customary one for strong embedding.

One has the following general result.

PROPOSITION. If $H$ possesses a strongly p-embedded subgroup, then one of the following holds:

(i) $H$ has a cyclic or generalized quaternion Sylow p-subgroup;

(ii) $\bar{H}=H / O_{p^{\prime}}(H)$ contains a simple normal subgroup $\bar{L}$ with $C_{\bar{H}}(\bar{L})=1$ such that $\bar{L}$ has a strongly $p$-embedded subgroup.

In effect, this proposition reduces the study of groups with a strongly $p$-embedded subgroup to the simple case. As you well know, Bender has classified all groups having a strongly embedded subgroup, the only simple ones being $L_{2}\left(2^{n}\right), S z\left(2^{n}\right), U_{3}\left(2^{n}\right), n \geqslant 2$. Recently, Aschbacher has extended Bender's argument to the case of groups of even order having a proper 2-generated core. Remarkably, Aschbacher's list includes only two additional quasisimple groups of 2-rank at least 3-namely, Janko's group $J_{1}$ of order 175,560 and the covering group $\hat{A}_{9}$ of $A_{9}$ by $Z_{2}$.

These two theorems of Bender and Aschbacher form the cornerstone for deriving contradictions when studying the centralizers of involutions in a simple group $G$. One wishes to prove that these centralizers have some property $X$. Assuming $X$ is false, one argues that the 1-generated core or 2-generated core of $G$ is proper. Then one quotes Bender or Aschbacher to conclude that $G \simeq L_{2}\left(2^{n}\right), S z\left(2^{n}\right), U_{3}\left(2^{n}\right)$, or $J_{1}$. Now one checks by inspection that $X$ holds in $G$.

For odd primes $p$, no corresponding classification theorem of groups with a strongly $p$-embedded subgroup exists. This would appear to present a fundamental obstacle in any attempt to analyze centralizers of elements of odd prime order $p$ in simple groups by the methods used to study centralizers of involutions, for we have no basic procedure for deriving contradictions.

However, there is a way out of this difficulty. It is based on the following result, which is not at all difficult to prove, and which I call the

THEOREM OF TRANSITION. Let $G$ be a group which possesses a strongly $p$-embedded subgroup $M, p$ odd. If every maximal 2-local subgroup of $G$ is 1 -generated for the prime $p$, then $M$ is strongly embedded in $G$ in the ordinary sense.

Later I shall explain how this result fits into the larger picture. We note here that by the preceding proposition the theorem will be applicable unless some maximal 2-local subgroup $H$ of $G$ either has a cyclic Sylow $p$-subgroup or $\bar{H}=H / O_{p^{\prime}}(H)$ is isomorphic to an automorphism group of a simple group $\bar{L}$ having a strongly $p$-embedded subgroup. In practice, of course, $\bar{L}$ will be of known type.

Among the known simple groups which possess a strongly $p$-embedded subgroup are obviously the analogues of the Bender groups-namely, $L_{2}\left(p^{n}\right)$, $U_{3}\left(p^{n}\right), n \geqslant 1$, and when $p=3$, the groups of Ree type of characteristic 3 . These seem, in fact, to include the only such groups of $p$-rank at least 3. There are, however, some further groups of $p$-rank 2; for example, the group 
$A_{2 p}$. Of course, any group $H$ with cyclic Sylow $p$-subgroup $P \neq 1$ and $O_{p}(H)=1$ has a strongly $p$-embedded subgroup; namely $N_{H}\left(\Omega_{1}(P)\right)$.

One should view the theorem of transition as follows: Either it is applicable or some maximal 2-local subgroup has a very restricted structure relative to the prime $p$. This will be particularly effective if $p=3$.

\section{THE PROGRAM}

Preliminary remarks. At the very outset, we face a serious technical problem. Obviously, we wish to proceed by induction to classify all simple groups, but unfortunately we have no reason to expect that our present list of simple groups is complete. This is underscored by Rudvalis' new group that has turned up in just the last two months. Fortunately, all the simple groups that have been discovered in recent years have internal structures very similar to those of previously known simple groups. This means that if in a particular argument of local analysis, some general property of the composition factors of the local subgroups (which will be known simple groups) is used, it is very likely that this property will continue to hold even if the list of known simple groups becomes expanded. This also indicates the advantage of proceeding in a somewhat axiomatic fashion, making explicit the specific general properties of the known simple groups that enter into the given arguments. One then simply has to check that any new simple group satisfies the given conditions to know that the argument remains valid in the presence of this group. I remark that the general properties of the known simple groups which primarily enter into the arguments are of the following types:

1. Balance;

2. Various kinds of generation;

3. General properties of centralizers of elements of prime-order-in particular, involutions;

4. Schur multipliers;

5. Automorphism groups.

I should add, however, that it is not always easy to state these conditions in such a way that they are valid for every known simple group-there always seem to be elusive exceptions. I want to emphasize that an integral part of this program is the prior verification of those general properties of the known simple groups which will be needed to make the local group theoretic analysis universally valid. Since there will be essentially no time for me to discuss techniques needed to treat any of the individual parts of the program, I shall have very little to say explicitly about these properties in outlining the program.

One final observation. A particular phase of the general classification problem may be inductive in its own right-for example, the classification of simple groups whose 2-local subgroups are all solvable. If, in such a situation, we feel that our present list of simple groups is complete, then there is clearly no point in proceeding axiomatically. Instead one should simply regard the problem as an independent classification theorem. Moreover, it is, in fact, necessary to do so, since in such problems more specific properties of the restricted composition factors of the local subgroups are usually required to 
carry through the analysis. This remark will apply to several individual parts of the program.

Since the complete classification of simple groups seems to be a long way off, it is clearly important to treat as many parts of the program as independent classification theorems as is possible.

With all these remarks in mind we shall, henceforth, consider a simple group $G$ all of whose local subgroups have composition factors of known type. Our goal then is to prove that $G$ itself is of known type. This is what the ultimate classification theorem will have to demonstrate.

The way to think of $G$ at the outset is not at all as a simple group, but rather as a group whose proper subgroup structure resembles that of a completely arbitrary group having composition factors of known type. Our task then is to use the assumption of simplicity to gradually force the internal structure of $G$ to resemble that of a known simple group. Basically, this assumption is used in the most naive way-it forces the subgroups of $G$ which we consider in our local analysis always to be proper.

Now finally for the outline itself, which will have sixteen parts.

I. Groups OF LOW 2-RANK. As I shall explain at the next step, the signalizer functor method does not begin to work well unless the 2-rank of $G$ is at least 5. It seems necessary, therefore, to treat the case in which $G$ has 2-rank at most 4 independently. This is a major undertaking which, in effect, has been in progress for about 15 years-and is still not completed.

At the present time, Harada and I have just completed the analysis in the case in which $G$ has sectional 2-rank at most 4. Because our hypothesis was imposed on the sectional 2-rank, we were able to prove our result by induction. However, if we assume only that the 2-rank of $G$ is at most 4, this is not an inductive condition, so it does not appear that one can easily achieve this classification result as an independent theorem. Probably one can prove that the sectional 2-rank of $G$ is at most, say, 12 and then classify all groups in which $r(G) \leqslant 12$, but this would be a painful task.

Harada has suggested a procedure which might work to attain an independent classification theorem. The analysis is to be divided into two parts:

Problem 1. Classify all groups in which all 2-local subgroups are either solvable or not 2-constrained.

This is an inductive condition and so it can be treated as an independent classification problem. The solution is to be carried out in 3 steps:

(A) All 2-local subgroups of $G$ are solvable.

This will require a generalization of large parts of the $N$-group paper.

(B) Some 2-local subgroup of $G$ is not 2-constrained and $m(G) \leqslant 4$.

This will require a generalization of the non 2-constrained portions of the sectional 2-rank problem-Parts III, IV, V, and VI of our paper. Of course, this much would have to be done anyhow if one wanted to classify simple groups of 2-rank at most 4.

(C) Some 2-local subgroup of $G$ is not 2-constrained and $m(G) \geqslant 5$. This will require the full application of the signalizer functor method which I shall describe below.

If Problem 1 is solved, we are reduced to the case in which our group $G$ of 2-rank at most 4 possesses a nonsolvable 2-constrained 2-local subgroup. 
Thus, we are led to

Problem 2. Determine the possible structures of a nonsolvable 2constrained group $H$ such that $m(H)<4$.

This is a noninductive problem, but it does seem amenable to attack. In Part II of the sectional 2-rank paper, we treated the corresponding problem when $r(H) \leqslant 4$. If Problem 2 could be solved, one would be in a position to apply the more precise fusion methods to force the structure of a Sylow 2-subgroup of $G$, in which case one would be able to quote some prior classification theorem.

II. THE SEMISIMPLICITY OF 2-LAYERS. Henceforth, we assume that $G$ has 2-rank at least 5. In particular, $\mathrm{SCN}_{3}(2)$ is nonempty.

Problem. Prove that the 2-layer $L_{2}\left(C_{G}(x)\right)$ of $C_{G}(x)$ is semisimple for every involution $x$ of $G$.

This will involve the use of the signalizer functor method which I would like to describe very briefly. The quasisimple components of $L\left(C_{G}(x)\right) / O\left(C_{G}(x)\right)$, being of known type, are either 2-balanced or isomorphic to $L_{2}(q), S L(2, q), q$ odd, $A_{n}$ or $\hat{A}_{n}$. The alternating groups present a technical problem, which can be handled, but which I prefer to avoid here. So let us here exclude the groups $A_{n}$ with $n \equiv 3(\bmod 8)$ from the set of possible components. Then all quasi-simple components will be 3-balanced as the groups $L_{2}(q), q$ odd, are 3-balanced.

As a consequence, one has

Proposition. If $T \in \mathcal{E}_{3}(G)$ and $a$ is an involution of $C_{G}(T)$, then

$$
\Delta_{G}(T) \cap C_{G}(a) \subseteq O\left(C_{G}(a)\right)
$$

Using this result, one can prove

Proposition. If $A$ is an elementary abelian 2-subgroup of $G$ of rank at least 5 and for $a$ in $A^{\#}$, we set

$$
\theta\left(C_{G}(a)\right)=\left\langle C_{G}(a) \cap \Delta_{G}(T) \mid T \in \mathcal{E}_{3}(A)\right\rangle,
$$

then

$$
\theta\left(C_{G}(x)\right) \cap C_{G}(y) \subseteq \theta\left(C_{G}(y)\right)
$$

for all $x, y$ in $A^{\#}$.

Thus, $\theta$ is a $A$-signalizer functor on $G$.

We remark that the assumption that $m(G) \geq 5$ is essential for the preceding result except in those cases in which the groups $L_{2}(q), q$ odd, do not occur among the components.

The signalizer functor theorem now yields

THEOREM. The group $W_{A}=\left\langle\theta\left(C_{G}(a)\right) \mid a \in A^{\#}\right\rangle=\left\langle\Delta_{G}(T) \mid T \in \mathcal{E}_{3}(A)\right\rangle$ is of odd order.

COROllary. Either $W_{A}=1$ or $N_{G}\left(W_{A}\right)$ is a proper subgroup of $G$.

There are now essentially three steps to proving the semisimplicity of $L_{2},\left(C_{G}(x)\right)$ for every involution $x$.

(A) Prove $W_{A}=1$. 
In the contrary case, prove that $N_{G}\left(W_{A}\right)$ is strongly embedded in $G$ or at least that $\Gamma_{S, 2}(G) \subseteq N_{G}\left(W_{A}\right)$ for some Sylow 2-subgroup $S$ of $G$. In carrying this out, specific generational properties of the components of the groups $L_{2}\left(C_{G}(x)\right)$ enter.

Thus, (A) implies that $\Delta_{G}(T)=1$ for all $T$ in $\varepsilon_{3}(A)$.

(B) Prove $\Delta_{G}(T)=1$ for all $T$ in $\varepsilon_{3}(G)$.

If each $T$ in $\mathcal{E}_{3}(G)$ lies in an element of $\mathscr{E}_{5}(G)$, (B) will follow from (A); so it is the remaining cases in which we are concerned.

(C) Prove $L_{2^{\prime}}\left(C_{G}(x)\right)$ is semisimple for every involution $x$ of $G$.

One must prove that $L_{2}\left(C_{G}(x)\right)$ centralizes $O\left(C_{G}(x)\right)$. In this analysis, the following general proposition, known as $L$-balance, is fundamental:

Proposition. If $x$ and $y$ are commuting involutions of $G$, then

$$
L_{2}^{\prime}\left(L_{2^{\prime}}\left(C_{G}(x)\right) \cap C_{G}(y)\right) \subseteq L_{2^{\prime}}\left(C_{G}(y)\right) .
$$

There is no time to go into further detail. Let me just say that this procedure has been successfully carried out in many specific classification problems.

III. STANDARD FORM IN ODD CHARACTERISTIC. Henceforth, we assume that $L_{2^{\prime}}\left(C_{G}(x)\right)$ is semisimple for every involution $x$ of $G$. We denote by $\mathcal{L}(G)$ the set of quasisimple components of $L_{2}\left(C_{G}(x)\right)$ as $x$ ranges over the involutions of $G$.

We first consider the case in which some element of $\mathcal{L}(G)$ is of Lie type of odd characteristic. Since certain groups of odd characteristic are isomorphic to groups of even characteristic or alternating groups, this presents a slight problem. From a technical point of view, it is probably best not to consider these "swing" groups as being of odd type. Allowing for this possibility, the goal under these circumstances can be stated as follows:

Problem. Prove that for some involution $x$ of $G, H=C_{G}(x)$ possesses a quasisimple normal subgroup $L$ of Lie type of odd characteristic such that $C_{H}(L)$ has 2-rank 1-i.e., $C_{H}(L)$ has cyclic or generalized quaternion Sylow 2-subgroups.

In such a case, we say that $H$ is in standard form with standard component L.

Clearly a result of this sort pins down $C_{G}(x)$ very tightly. We note that John Walter's work on this problem indicates that one can most likely force the approximate structure of the centralizers of all the involutions of a maximal elementary 2-subgroup of $G$ containing $x$.

The reason we can expect such a strong conclusion in this case is due to the special way in which groups of Lie type of odd characteristic are generated. In fact, if $T$ is any four group acting on such a quasisimple group $K$, one has

$$
K=\left\langle C_{K}(t)^{\prime} \mid t \in T^{\#}\right\rangle .
$$

Indeed, except in certain cases when $K$ has low Lie rank, one actually has

$$
K=\left\langle L\left(C_{K}(t)\right) \mid t \in T^{\#}\right\rangle .
$$

Using these properties of generation together with $L$-balance and assuming the desired conclusion is false, the aim of the analysis is to prove

(A) $V_{A}=\left\langle\Lambda\left(C_{G}(a)\right) \mid a \in A^{\#}\right\rangle$ is a semisimple group. Here $\Lambda\left(C_{G}(a)\right)$ 
denotes the product of those components of $L\left(C_{G}(a)\right)$ that are of Lie type of odd characteristic.

(B) $N_{G}\left(V_{A}\right)$ is strongly embedded in $G$.

The spirit as well as specific portions of this analysis is very similar to that involved in proving the semisimplicity of 2-layers. Therefore, it is reasonable to include it in what we may call the general "signalizer functor" method.

IV. CLASSIFICATION OF GROUPS OF ODD TYPE. Once we have the centralizer $H$ of an involution in standard form with standard component $L$ of Lie type of odd characteristic, we are very close to the known characterization theorems of these groups by centralizers of their involutions. Of course, $C_{H}(L)$ may not have the correct form for the known group, but its structure is very limited as $C_{H}(L)$ has cyclic or generalized quaternion Sylow 2subgroups. Likewise, $H / L C_{H}(L)$ may be wrong. However, this group is isomorphic to a group of outer automorphisms of $L$ and so is also very restricted.

Problem. Generalize the known characterization theorems for groups of Lie type of odd characteristic to the standard form case.

V. QUASI-STANDARD FORM. At this stage we assume that no element of $\mathcal{L}(G)$ is of Lie type of odd characteristic. When $\mathscr{L}(G)$ is nonempty, we would obviously like to generalize the analysis of the odd characteristic case. Unfortunately, the groups of Lie type of even characteristic, the alternating, and sporadic groups do not have such nice generational properties, so it is not clear how far the signalizer functor method can be pushed.

Out of our work on groups with Sylow 2-subgroups of class 2, Gilman and I have managed to extend one of our arguments to give a general first approximation.

For some involution $x$ of $G$, if $H=C_{G}(x)$, then the 2-layer $L$ of $H$ is quasisimple and $m\left(C_{H}(L)\right) \leqslant m(L)+2$.

One may conjecture

Problem. Prove $x$ and $L$ exist so that $C_{H}(L)$ has 2-rank at most 2.

This problem has not been considered very seriously and it is not clear how reasonable this conjecture is. In any event, let us say that $H$ is in quasi-standard form when $L$ exists with $C_{H}(L)$ of low 2-rank.

VI. Central involutions. Remarkably if $G$ is of Lie type of even characteristic or a sporadic group and the 2-rank of $G$ is at least 5, then

$$
L_{2^{\prime}}\left(C_{G}(x)\right)=L\left(C_{G}(x)\right)=1
$$

for all involutions $x$ in the center of a Sylow 2-subgroup. This suggests the following closely related problem:

Problem. Prove that the centralizer of every central involution is 2constrained.

Presumably in the contrary case, one will try to argue that $C_{G}(x)$ is in quasi-standard form for some central involution $x$ of $G$ and if $L$ is the corresponding component of $C_{G}(x)$ that $N_{G}(L)$ is strongly embedded in $G$. At the least, the second assertion will involve an analysis of the group $N_{G}(A)$, where $A$ is an elementary abelian 2-subgroup of $C_{G}(x)$ of maximum rank.

VII. Classification of ALTERNATING GROUPS. Presumably if some element of $\mathcal{L}(G)$ is an alternating group of not too low a degree, this should be 
enough to force $G$ itself to be an alternating group. Clearly, the goal would be to prove that $x$ exists with $H=C_{G}(x)$ in quasi-standard form and with component $L$ an alternating group. If one can achieve this, one would be close to Kondo's characterization of the alternating groups.

Problem. Characterize the alternating groups along these lines.

The case in which all elements of $\mathcal{L}(G)$ are assumed to be alternating groups will obviously be of great interest.

VIII. SOME SPORADIC GROUPS. We now reach a very interesting situation:

(a) $C_{G}(x)$ is 2-constrained for every central involution $x$;

(b) Every element of $\mathcal{L}(G)$ is of Lie type of characteristic 2 or a sporadic group;

(c) $C_{G}(y)$ is in quasi-standard form for some involution $y$.

For example, these conditions hold in Rudvalis' group with $C_{G}(y) \cong Z_{2} \times$ $Z_{2} \times S z(8)$ and in the smallest Fischer group with $C_{G}(y) /\langle y\rangle \cong U_{6}(2)$.

Problem. Determine all groups which satisfy (a), (b), and (c).

Very special cases of this problem are treated in Part I of the 3'-problem, the abelian Sylow 2-group problem, Collins' characterization of the Suzuki groups, and in the class 2 Sylow 2-group problem.

The problem is actually larger than it appears, for there exist a great many "almost simple" groups satisfying these conditions. Let $G^{*}$ be an extension of a group $K$ of Lie type of characteristic 2 by an automorphism $y$ of period 2 . Then $C_{G^{*}}(y)=L \times\langle y\rangle$, where $L=C_{K}(y)$; and for most choices of $K$ and $y, L$ will be a simple group of Lie type of characteristic 2 . To rule out these cases in our situation, it will clearly be necessary to pinpoint a Sylow 2-subgroup of $G$ and then by transfer force $G$ to have a normal subgroup of index 2.

In both this problem and the preceding alternating group situation, one will again be concerned with $C_{G}(x)$ in quasi-standard form and the interrelations between the groups $N_{G}(L)$ and $N_{G}(A)$, where $L$ is the component of $C_{G}(x)$ and $A$ is an elementary abelian 2-subgroup of $C_{G}(x)$ of maximal rank.

A BRIEF DIGRESSION. This list of problems may seem overwhelming. And even if they are all solved, where does it put us? We shall have reached the stage in which the centralizer of every involution of $G$ is 2-constrained. By some general results, every 2-local subgroup of $G$ is then 2-constrained and each has a trivial core. Thus, our simple group $G$ of 2-rank at least 5 will be what I call of

\section{CHARACTERISTIC 2 TYPE,}

inasmuch as the simple groups of Lie type of characteristic 2 have 2-local subgroups which satisfy these conditions.

However, from what we have seen in the $N$-group paper and heard about the 3'-problem, the major portion of our work still lies ahead of us; so it all may seem very discouraging. Therefore, to give you sustenance for the long journey ahead, let me list some specific cases in which this phase of the program has been successfully completed or nearly completed.

1. Groups with abelian Sylow 2-subgroups;

2. Groups with Sylow 2-subgroups of class 2;

3. 3'-groups;

4. Groups of order $2^{a} \cdot 3^{b} \cdot 13^{c}$. 
One of my $\mathrm{Ph} . \mathrm{D}$. students is working on the problem of classifying groups of order $2^{a} \cdot 3^{b} \cdot p^{c}$. We happen to have begun with the case $p=13$, since in that case there is only one known simple group with such an order, namely $L_{3}(3)$, and it happens to be 1-balanced.

In each of these four problems, one has been able to prove or is very close to proving at the present time that a minimal counterexample to a classification theorem is a group of characteristic 2 type. Of course, in the abelian problem, theorems of Feit or Suzuki complete the classification at once; but in the other problems, the proofs are far from over at this point.

5. The work of John Walter in the case in which some element of $\mathcal{L}(G)$ is of odd type can be viewed as a successful execution of parts of the above program.

Finally I wish to mention a general result that Harris and I have recently obtained which utilizes these methods and which I hope will be useful in further classification problems.

We say that a group $G$ has product fusion provided: $S_{2}$

(a) A Sylow 2-subgroup $S$ of $G$ is the direct product of subgroups $S_{1}$ and

(b) No element of $S_{i}$ is conjugate in $G$ to an element of $S-S_{i}, i=1,2$.

Clearly $G$ has product fusion if $G=G_{1} \times G_{2}$ with $S_{i}$ a Sylow 2-subgroup of $G_{i}, i=1,2$; which explains our terminology.

Our result is essentially the converse assertion.

THEOREM. Let $G$ be a group with product fusion (relative to the decomposition $S=S_{1} \times S_{2}$ ) and assume that every simple group involved in a 2-local subgroup of $G$ is either 3-balanced or an alternating group. If $G$ has no nontrivial normal subgroup of odd order or odd index, then

$$
\boldsymbol{G}=G_{1} \times G_{2}
$$

where $S_{i}$ is a Sylow 2-subgroup of $G_{i}, i=1,2 .^{14}$

Presumably our assumption on balance holds in every specific inductive classification problem. Actually, we require this assumption only for the composition factors of a certain inductive set of subgroups of the 2-local subgroups.

I hope these results will give you encouragement to consider seriously some of the above problems and to bear with me as I present the balance of the program.

IX. THIN GROUPS. It remains now to present a procedure for analyzing groups of characteristic 2 type. Here the sole guidelines come from the $N$-group and $3^{\prime}$-problems and whatever I have to say is based on my understanding of these classification theorems. Although Thompson's actual proofs are carried out in the form of reductions to tighter and tighter situations, it will be preferable in describing the overall program to reverse this procedure and build step-by-step from the special to the general.

\footnotetext{
${ }^{14}$ We do not explicitly treat the cases in which $S_{1}$ and $S_{2}$ are each dihedral, quasi-dihedral, wreathed, homocyclic abelian of rank 2 , or of type $U_{3}(4)$, some of which have not yet been handled. Mason is presently working on the remaining cases, but until this work is done, our proof will actually be incomplete.
} 
The principal subdivisions in Thompson's analysis are determined by the $p$-ranks of the 2-local subgroups of $G$ for odd primes $p$. Let us then define the 2-local p-rank of $G$, for any odd prime $p$, to be the maximum of $m_{p}(H)$ as $H$ ranges over the 2-local subgroups of $G$.

Janko has introduced the term thin for any group whose 2-local $p$-rank is at most 1 for all odd $p$-equivalently, whose 2-local subgroups have cyclic Sylow $p$-subgroups for all odd $p$. A major chapter of the $N$-groups and $3^{\prime}$-papers deals with the case in which $G$ is thin. Moreover, Janko has generalized Thompson's $\mathrm{N}$-group argument to obtain a classification of thin groups with solvable 2-local subgroups.

Problem. Classify all thin groups.

At least this very important problem can be treated as an independent inductive problem. Thompson's and Janko's work suggest that the main tools involved will be the weak closure of abelian 2-groups, double factorization lemmas, and the centralizers of elements of odd order. However, Janko's preliminary investigations of this general problem indicate that some difficult minimal cases exist when $L_{2}\left(2^{n}\right)$ is a composition factor of a 2-local subgroup.

$X$. GROUPS WITH A STRONGLY $p$-EMBEDDED SUBGROUP, $p$ ODD. We can, therefore, assume that $G$ is not thin, in which case the 2-local $p$-rank of $G$ is at least 2 for some odd $p$. The basic additional tool which Thompson utilizes to treat this case is a uniqueness theorem for such primes $p$. In our present terminology, this is equivalent to saying that $G$ has a proper 2-generated $p$-core-which will hold, in particular, if $G$ has a strongly $p$-embedded subgroup.

We remark that Thompson actually divides the analysis into two cases-according as the 2-local $p$-rank is 2 or at least 3 . The first case requires a special analysis of the 2-local and $p$-local subgroups of $G$. However, in the $N$-group paper, the second case, which is the general case, is essentially a consequence of general arguments in the odd order paper.

Here then is an important general problem, which we prefer to divide into two parts.

Problem 1. If the 2-local 3-rank of $G$ is at least 3, prove that $G$ does not possess a strongly 3 -embedded subgroup.

Problem 2. If the 2-local 3-rank of $G$ is at most 2, prove that $G$ does not possess a strongly $p$-embedded subgroup if the 2-local $p$-rank of $G$ is at least 3 .

It may be necessary to generalize these problems to the case of a proper 2-generated 3-core and $p$-core.

Clearly the theorem of transition is directly applicable and implies that some maximal 2-local subgroup $H$ of $G$ is not 1-generated for 3 or $p$, as the case may be. We have noted earlier the restricted nature of such a group $H$, particularly for the prime 3 . It is for this reason that we have made the above subdivision, for in the second case the composition factors of $H$ are again highly restricted no matter what value $p$ has inasmuch as $H$ does not contain a subgroup of type $(3,3,3)$.

The problem here is still to prove that our strongly $p$-embedded subgroup is strongly embedded in the ordinary sense, but Thompson's work indicates that to prove this will again require an analysis of the weak closure of abelian 2-subgroups of $G$. 
XI. THE SIGNALIZER FUNCTOR METHOD FOR ODD PRIMES. Henceforth, we assume that $G$ does not possess a strongly $p$-embedded subgroup and, if necessary, does not have a proper 2-generated $p$-core, where $p=3$ or $p>3$ as in the preceding section. What are the implications of this assumption? To answer this, let us ask the corresponding question for the prime 2: What was the implication of the fact that $G$ did not have a proper 2-generated core?

Using the signalizer functor method, we hoped to obtain the following conclusions when $m(G) \geqslant 5$ :

(A) $L_{2^{\prime}}\left(C_{G}(x)\right)$ is semisimple for every involution $x$ of $G$;

(B) One of the following holds:

(1) $G$ is of characteristic 2 type (2-locals are 2-constrained and have trivial cores);

(2) $C_{G}(x)$ is in standard form with standard component of Lie type of odd characteristic for some involution $x$;

(3) No element of $\mathcal{L}(G)$ is of Lie type of odd characteristic and $C_{G}(x)$ is in quasi-standard form for some involution $x$.

Except in the low rank cases, the proofs of these results are all very formal in nature and only use the fact that our prime is 2 in a single way-namely, that $O_{2^{\prime}}\left(C_{G}(x)\right)=O\left(C_{G}(x)\right)$ is of odd order and hence solvable. Because of this, our original signalizer functor $\theta$ was solvable in the sense of Goldschmidt and so the signalizer functor theorem was applicable.

Unfortunately, if $x$ is an element of order $p, p$ odd, $O_{p^{\prime}}\left(C_{G}(x)\right)$ need not be solvable, so in general, the corresponding signalizer functor $\theta$ will not be solvable. There are actually two possible ways out of this difficulty.

Problem 1. Prove a nonsolvable signalizer functor theorem.

In fact, we have carried through a large portion of the proof of such a result. We note that there is a decided advantage here in having $p=3$, since in this case the only nonsolvable composition factors of $O_{p^{\prime}}\left(C_{G}(x)\right)$ will be Suzuki groups.

Problem 2. Show in the present context that solvable signalizer functors are sufficient.

The point is that because $G$ is of characteristic 2 type, it may be enough to work with $S\left(O_{p^{\prime}}\left(C_{G}(x)\right)\right)$, the unique largest normal solvable subgroup of $O_{p^{\prime}}\left(C_{G}(x)\right)$.

Once this problem is successfully handled, the entire signalizer functor machinery can be bodily carried over to the case of our odd prime $p$ provided the $p$-rank of $G$ is large enough. Presumably, the low $p$-rank cases will require, as in Thompson's cases, a mixture of 2-local and $p$-local analysis to obtain the desired results.

Problem 3. Prove that $L_{p^{\prime}}\left(C_{G}(x)\right)$ is semisimple for every element $x$ of $G$ or order $p$.

Now define $\mathcal{L}_{p}(G)$ to be the set of quasisimple components of $L_{p^{\prime}}\left(C_{G}(x)\right)$ as $x$ ranges over the elements of $G$ of order $p$.

Problem 4. Prove that one of the following holds:

(1) $G$ is of characteristic $p$ type ( $p$-locals are $p$-constrained and have no nontrivial normal $p^{\prime}$-subgroups).

(2) $C_{G}(x)$ is in standard form with standard component a group of Lie type of characteristic 2 or a sporadic group for some $x$ of order $p$; 
(3) No element of $\mathcal{L}(G)$ is of Lie type of characteristic 2 or a sporadic group and $C_{G}(x)$ is in quasi-standard form for some $x$ of order $p$.

Because $G$ is of characteristic 2 type to begin with, there are indications that the possibilities that may occur under (3) will be very limited.

XII. GROUPS OF CHARACTERISTIC $p$ TYPE. It remains to consider the three alternatives of Problem 4 of the last section. Suppose first that $G$ is of characteristic $p$ type (as well, of course, as of characteristic 2 type). We postpone to the next two sections the case in which $m_{p}(G)=2$ and so we assume, in addition, that $G$ has $p$-rank at least 3.

Thompson's analysis of this case in the $N$-group situation constituted a major undertaking and led to his beautiful characterizations of the groups $G_{2}(3)$ and $S_{p}(4,3)$. Surprisingly enough, so far as I can tell, these two groups are the only ones among the presently known simple groups that satisfy our more general conditions. This suggests

Problem. Prove that $G_{2}(3)$ and $S_{p}(4,3)$ are the only groups which are simultaneously of characteristic 2 and $p$ type (with $m_{p}(G) \geqslant 3$ ).

If every maximal $p$-local subgroup of $G$ were $p$-stable, Glauberman's $Z J$-Theorem would lead at once to the conclusion that $G$ had a strongly $p$-embedded subgroup, which we are here assuming is not the case. Hence, some maximal $p$-local subgroup $H$ of $G$ is not $p$-stable. The structure of non $p$-stable $p$-constrained groups is closely related to the theory of quadratic pairs. If Thompson's classification of quadratic pairs for $p \geqslant 5$ can be extended to the case $p=3$, one may be able to analyze the structure of $H$ without invoking our induction assumption on the proper subgroups of $G$.

In any event, it is clear that one should thoroughly digest Thompson's characterizations of $G_{2}(3)$ and $S_{p}(4,3)$ before undertaking this fascinating problem.

XIII. QUASITHIN GROUPS. In treating the remaining problems, it will undoubtedly be best to separate out the cases in which $G$ has low 2-local 3-rank. It is too early to tell whether "low" should be regarded as 2, 3, or 4 . Hopefully, it will turn out to be the smallest of the values. Clearly the classification of $3^{\prime}$-groups is just the first step towards a solution of this problem.

We shall divide the problem into two parts. Let us say that a group is quasithin if its 2-local subgroups have $p$-rank at most 2 for all odd $p$ (or at most 3 or 4 , if need be).

Unfortunately, the quasithin groups are not an inductive family, since just as in the case of the prime 2 the $p$-rank may increase under homomorphic images. Probably there is an analogue of MacWilliams Theorem which asserts that the sectional $p$-rank of a group is at most 3 (or perhaps 4) if its $p$-rank is at most 2. But then if one used the sectional $p$-rank condition for 2-local subgroups in defining quasithin groups one would obtain an inductive family of groups which included the groups in which we are interested.

In any event, we have

Problem. Classify all quasithin simple groups of characteristic 2 type.

Clearly Thompson's work in the $N$-group and $3^{\prime}$-cases plus any results one obtains about thin groups will be relevant to this problem.

XIV. GROUPS OF LOW 2-LOCAL 3-RANK. To complete the classification of 
groups of 2-local 3-rank at most 2, one would have to consider:

Problem. If $G$ is a group of 2-local 3-rank at most 2, prove that $G$ is quasithin.

Suppose the 2-local $p$-rank of $G$ is at least 3 for some $p$. If we assume that the case in which $G$ is of characteristic $p$ type has already been dealt with, it will presumably follow that the centralizer of some element of order $p$ will be in standard or quasi-standard form. Because of the severe limitation on the elements of $\mathcal{L}_{p}(G)$ (due to our assumption on the prime 3 ), there is a good chance that one will be able to construct a strongly $p$-embedded subgroup and thus derive a contradiction in these cases.

XV. Centralizers of 3-elements IN STANDard Form. In view of the preceding discussion, we can suppose henceforth that the 2-local 3-rank of $G$ is at least 3. Thus, our prime $p$ above can be taken to be 3. Moreover, we can suppose that $G$ is not of characteristic 3 type. We are, therefore, left with the cases in which the centralizer of some element of order 3 is in quasi-standard or standard form.

I know of no example of a group of characteristic 2 type in which the centralizer of an element of order 3 is in quasi-standard form with component of Lie type of odd characteristic or an alternating group, so perhaps this case cannot arise. As remarked before, the fact that $G$ is of characteristic 2 type will severely limit the possible components in such a case and hopefully one will, as in the preceding section, be able to construct a strongly 3-embedded subgroup to obtain a contradiction. Thus, we have

Problem. Prove that the centralizer of some element of order 3 is in standard form with standard component of Lie type of characteristic 2 or a sporadic group.

XVI. Classification of simple groups of characteristic 2 type. We come at last to the final step in the program.

Problem. Classify simple groups of characteristic 2 type in which, for some element $x$ of order $3, C_{G}(x)$ is in standard form with standard component of Lie type of characteristic 2 or a sporadic group.

We assume, in addition, of course, that the 2-local 3-rank of $G$ is at least 3 and that $G$ does not possess a strongly 3-embedded subgroup (or a proper 2-generated 3-core).

The idea here, at least for the groups of Lie type, is to build up the $(B, N)$-pair structure from the centralizer of an element of order 3 in much the same way that one classified groups of Lie type of odd characteristic by the centralizers of involutions. One will protest at once that a fundamental tool in that endeavor was Glauberman's $Z^{*}$-theorem without which it would have been impossible to have analyzed the fusion of involutions of $G$-and no analogue of the $Z^{*}$-theorem is in sight for the prime 3 .

Hence we have the following problem:

Subproblem. Prove that $x$ is not isolated in $C_{G}(x)$ with respect to $G$.

If one thinks a bit about this problem, one will soon see that the way to establish this result is to argue in the contrary case that $G$ must have a strongly 3-embedded subgroup.

Clearly without a solution of the subproblem we shall not be able to attack the fusion of elements of order 3 in $G$. 
In order to test the feasability of this procedure for classifying groups of characteristic 2 type, I suggested that one of my Ph.D. students, Robert Miller, attempt a characterization of $L_{4}\left(2^{n}\right), n$ even, by the structure of the centralizer $H$ of an element $x$ of order 3 in the center of a Sylow 3-subgroup (in which case $H \cong G L\left(3,2^{n}\right)$ ). The condition, $n$ even, is needed to insure that the 2-local 3-rank of $G$ is at least 3. To avoid complications of a different nature, I allowed him to assume that any simple section of $G$ of 3-rank at most 2 was of known type.

Under the assumption that $x$ is not isolated in $H$ with respect to $G$, Miller has proved that a simple group $G$ satisfying these conditions is necessarily isomorphic to $L_{4}\left(2^{n}\right)$. In addition, he has made considerable progress on the prior problem of showing that, in fact, $x$ is not isolated in $H$. However, this phase of his work is at present incomplete.

Miller's thesis indicates to me that the suggested procedure is a reasonable one for classifying groups of characteristic 2 type. I remark that his characterization theorem does not proceed directly to the construction of a $(B, N)$ pair, but rather to Suzuki's characterization of $L_{4}\left(2^{n}\right)$ by the centralizers of involutions. If this turns out to be typical, it will mean that the existing characterizations of the known groups of characteristic 2 type by centralizers of involutions will play an essential role.

I think these comments explain fully how I conceive the role of centralizers of elements of order 3 in the classification of simple groups-as the final movement of an elaborate symphony. Thompson has said that our work to date represents only a prelude. Hopefully, the program I have described here will bear some relation to the full symphony that remains to be written.

\section{BIBLIOGRAPHY ${ }^{15}$}

1. J. L. Alperin, Sylow intersections and fusion, J. Algebra (1967), 222-241.

2. __ Sylow 2-subgroups of rank 3, Gainesville conference, pp. 3-5 (cf. 170).

3. J. L. Alperin, R. Brauer and D. Gorenstein, Finite groups with quasi-dihedral and wreathed Sylow 2-subgroups, Trans. Amer. Math. Soc. 151 (1970), 1-261.

4. __ Finite simple groups of 2-rank two, Scripta Math. 29 (1973), 191-214.

5. J. L. Alperin and D. Gorenstein, The multiplicators of certain simple groups, Proc. Amer. Math. Soc. 17 (1966), 515-519.

6. M. Aschbacher, Finite groups with a proper 2-generated core, Trans. Amer. Math. Soc. 197 (1974), 87-112.

7. Finite groups generated by odd transpositions. I, II, III, IV, Math. Z. 127 (1972),

45-56; J. Algebra 26 (1973), 451-459; 26 (1973), 460-478; 26 (1973), 479-491.

8. ___ On finite groups of component type, Illinois J. Math. 19 (1975), 87-115.

9. ___ Tightly embedded subgroups of finite groups, J. Algebra 42 (1976), 85-101.

10. $\ldots$ A characterization of Chevalley groups over fields of odd characteristic, Ann. of Math. 106 (1977), 353-398.

11. __ On the failure of Thompson factorization in 2-constrained groups (to appear).

12. _ـ A factorization theorem for 2-constrained groups (to appear).

13. __ A pushing up theorem for characteristic 2 type groups (to appear).

14. _ A variation of the $C(G ; T)$ theorem for 2-constrained groups (to appear).

15. M. Aschbacher and G. Seitz, Involutions in Chevalley groups over fields of even order, Nagoya Math. J. 63 (1976), 1-91.

${ }^{15}$ This bibliography is not intended as a complete list of references on finite simple groups, but is limited to articles and books referred to in the text. 
16. On groups with a standard component of known type, Osaka J. Math. 13 (1976), 439-482.

17. B. Baumann, Über endliche Gruppen mit einer $z u L_{2}\left(2^{n}\right)$ isomorphen Faktorgruppe (to appear).

18. H. Bender, Endliche zweifach transitive Permutationsgruppen, deren innolutionen keine Fixpunkte haben, Math. Z. 104 (1968), 175-204.

19. _ Transitive Gruppen gerader Ordnung, in denen jede Involution genau einen Punkt festlässt, J. Algebra 17 (1971), 527-554.

20. __ On groups with abelian Sylow 2-subgroups, Math. Z. 117 (1970), 164-176.

21. __ On the uniqueness theorem, Illinois J. Math. 14 (1970), 376-384.

22. __, Goldschmidt's 2-signalizer functor theorem, Israel J. Math. 22 (1975), 208-213.

23. _ On groups with dihedral Sylow 2-subgroups (to appear).

24. N. Blackburn, On a special class of p-groups, Acta. Math. 100 (1958), 45-92.

25. , Generalizations of certain elementary theorems on p-groups, Proc. London Math. Soc. 11 (1961), 1-22.

26. A. Borel and J. Tits, Éléments unipotents et sousgroupes paraboliques de groupes réductifs. I, Invent. Math. 12 (1971), 95-104.

27. R. Brauer, On the structure of groups of finite order, Proc. Internat. Congr. Math. Amsterdam (1954), vol. 1, pp. 209-217, Noordhoff, Groningen, North-Holland, Amsterdam.

28. __ On finite Desarguesian planes. I, II, Math. Z. 90 (1966), 117-151.

29. __ Some applications of the theory of blocks of characters of finite groups. I, II, III, J. Algebra 1 (1964), 152-167; 307-334; 3 (1966), 225-255.

30. R. Brauer and K. Fowler, On groups of even order, Ann. of Math. 62 (1955), 565-583.

31. R. Brauer and C. Sah, Finite groups, a symposium, edited by R. Brauer and C. Sah, Benjamin, New York, 1969.

32. R. Brauer and M. Suzuki, On finite groups of even order whose 2-Sylow subgroup is a quaternion group, Proc. Nat. Acad. Sci. U.S.A. 45 (1959), 1757-1759.

33. R. Brauer, M. Suzuki and G. Wall, $A$ characterization of the one-dimensional unimodular groups over finite fields, Illinois J. Math. 2 (1958), 718-745.

34. N. Burgoyne, Finite groups with Chevalley-type components (to appear).

35. __ Elements of order 3 in Chevalley groups of characteristic 2 (unpublished).

36. M. Burgoyne and P. Fong, The Schur multipliers of the Mathieu groups, Nagoya Math. J. 27 (1966), 733-745; correction, 31 (1968), 297-304.

37. M. Burgoyne and C. Williamson, Centralizers of involutions in Chevalley groups of odd characteristic (to appear).

38. __ Semisimple classes in Chevalley groups (to appear).

39. R. Carmichael, Introduction to the theory of groups of finite order, Ginn, Boston and New York, 1937.

40. R. Carter and P. Fong, The Sylow 2-subgroups of the finite classical groups, J. Algebra 1 (1964), 139-151.

41. C. Chevalley, Sur certains groupes simples, Tôhoku Math. J. 7 (1955), 14-66.

42. J. Conway, $A$ group of order 8, 315, 553, 613, 086, 720, 000, Bull. London Math. Soc. 1 (1969), 79-88.

43. __ Three lectures on exceptional groups, Higman-Powell, 215-247 (cf. 118).

44. J. Conway and D. Wales, Construction of the Rudvalis group of order 145, 926, 144, 000, J. Algebra 27 (1973), 538-548.

45. B. Cooperstein, An enemies list for factorization theorems (to appear).

46. B. Cooperstein and G. Mason, Some questions concerning the representation of Chevalley groups of characteristic 2 (to appear).

47. C. Curtis, Central extensions of groups of Lie type, Crelle J. 220 (1965), 174-185.

48. P. Dembowski and A. Wagner, Some characterizations of finite projective space, Arch. Math. 11 (1960), 465-469.

49. U. Dempwolff, On the extensions of an elementary group of order $2^{5}$ by $G L(5,2)$, Rend. Sem. Mat. Univ. Padova 48 (1972), 359-364.

50. A. Dodd and B. Eckmann, Seminar on algebraic groups and related finite groups, Lecture Notes in Math., Springer-Verlag, Berlin and New York, 1970.

51. __ Proceedings of Second International Conference on the Theory of Groups, Lecture Notes in Math., Springer-Verlag, Berlin and New York, 1973. 
52. W. Feit, On a class of doubly transitive permutation groups, Illinois J. Math. 4 (1960), 170-186.

53. A characterization of the simple groups $\operatorname{SL}\left(2,2^{a}\right)$, Amer. J. Math. 82 (1960), 281-300; correction, 84 (1962), 201-204.

54. 633-683. , On integral representations of finite groups, Proc. London Math. Soc. 29 (1974),

55. W. Feit, M. Hall and J. Thompson, Finite groups in which the centralizer of any nonidentity element is nilpotent, Math. Z. 74 (1960), 1-17.

56. W. Feit and G. Higman, On the nonexistence of certain generalized polygons, J. Algebra 1 (1964), 114-131.

57. W. Feit and J. Thompson, Solvability of groups of odd order, Pacific J. Math. 13 (1963), 775-1029.

58. D. Fendel, A characterization of Conway's group.3, J. Algebra 24 (1973), 159-196.

59. B. Fischer, $A$ characterization of the symmetric groups on 4 and 5 letters, J. Algebra 3 (1966), 88-98.

60. __ Distributiv Quasigruppen endlicher Ordnung, Math. Z. 83 (1964), 267-303.

61. _ Finite groups admitting a fixed point free automorphism of order $2 p$. I, II, J. Algebra 3 (1966), 98-114; 5 (1967), 25-40.

62. __ Finite groups generated by 3-transpositions, University of Warwick (preprint).

63. ___ Finite groups generated by 3-transpositions, Invent. Math. 13 (1971), 232-246.

64. __ Evidence for the existence of a new $(3,4)$-transposition group (unpublished).

65. P. Fong and G. Seitz, Groups with $a(B, N)$-pair of rank 2. I, II, Invent. Math. 21 (1973), 1-57; 24 (1974), 191-239.

66. P. Fong and W. Wong, $A$ characterization of the finite simple groups $P S p(4, q), G_{2}(q)$, $D_{4}^{2}(q)$. I, II, Nagoya Math. J. 36 (1969), 143-184; 39 (1970), 39-79.

67. R. Foote, Finite groups with maximal 2-components of type $L_{2}(q), q$ odd (to appear).

68. R. Gilman and D. Gorenstein, Finite groups with Sylow 2-subgroups of class two. I, II, Trans. Amer. Math. Soc. 207 (1975), 1-101; pp. 103-125.

69. G. Glauberman, A characteristic subgroup of a p-stable group, Canad. J. Math. 20 (1968), 1101-1135.

70. ___ Subgroups of finite groups, Bull. Amer. Math. Soc. 73 (1967), 1-12.

71. __ Central elements in core-free groups, J. Algebra 4 (1966), 403-420.

72. __ On solvable signalizer functors in finite groups, Proc. London Math. Soc. 33 (1976), $1-27$.

73. __ Global and local properties of simple groups, Higman-Powell, 1-64 (cf. 118).

74. _ Isomorphic subgroups of finite p-groups. I, II, Canad. J. Math. 23 (1971), 983-1022; 1023-1039.

75. _ Factorizations in local subgroups of finite groups, Expository lectures, CBMS conference, Univ. of Minnesota, Duluth, 1976.

76. D. Goldschmidt, A conjugation family for finite groups, J. Algebra 16 (1970), 138-142.

77. __ Solvable signalizer functors on finite groups, J. Algebra 21 (1972), 137-148.

78. __ 2-signalizer functors on finite groups, J. Algebra 21 (1972), 321-340.

79. __ 2-fusion in finite groups, Ann. of Math. 99 (1974), 70-117.

80. __ Strongly closed 2-subgroups of finite groups, Ann. of Math. (2) 102 (1975), 475-489.

81. _ Pushing up $A_{n}$ and $\Sigma_{n}$ (unpublished).

82. D. Gorenstein, On the centralizers of involutions in finite groups, J. Algebra 11 (1969), 243-277.

83. __ The flatness of signalizer functors on finite groups, J. Algebra 13 (1939), 509-512.

84. __ Finite groups, Harper and Row, New York, 1968. 5-13.

85. __ On finite simple groups of characteristic 2 type, Inst. Hautes Ėtudes Sci. 36 (1969),

86. D. Gorenstein and K. Harada, Finite groups whose Sylow 2-subgroups are the direct product of two dihedral groups, Ann. of Math. 95 (1972), 1-54.

87. On finite groups with Sylow 2-subgroups of type $A_{n}, n=8,9,10,11$, Math. Z. 117 (1970), 207-237.

88. Math. Soc. 77 (1971), 829-862. 
89. , Finite groups whose 2-subgroups are generated by mast 4 elements, Mem. Amer. Math. Soc. No. 147 (1974), pp. 1-464.

90. D. Gorenstein and M. Harris, A characterization of the Higman-Sims simple group, J. Algebra 24 (1973), 565-590.

91. Finite groups with product fusion, Ann. of Math. 101 (1975), 45-87.

92. D. Gorenstein and R. Lyons, Non-solvable signalizer functors on finite groups, Proc. London Math. Soc. 35 (1977), 1-33.

93. D. Gorenstein and J. Walter, On the maximal subgroups of finite simple groups, J. Algebra 1 (1964), 168-231.

94. (1965), 85-151, 218-270, 354-393.

95. C_ Centralizers of involutions in balanced groups, J. Algebra 20 (1972), 284-319.

96. ㄴ The ж-layer of a finite group, Illinois J. Math. 15 (1971), 555-564.

97. __ Balance and generation in finite groups, J. Algebra 33 (1975), 224-287.

98. R. Griess, Schur multipliers of finite simple groups of Lie type, Trans. Amer. Math. Soc. 183 (1973), 355-421.

99. ___ Schur multipliers of some sporadic simple groups, J. Algebra 20 (1972), 320-349.

100. $\longrightarrow$ On the subgroup structure of the group of order $2^{46} \cdot 3^{20} \cdot 5^{9} \cdot 7^{6} \cdot 11^{2} \cdot 13^{3} \cdot 17 \cdot 19$. $23 \cdot 29 \cdot 31 \cdot 41 \cdot 47 \cdot 59 \cdot 71$ (to appear).

101. , The structure of the "monster" simple group, Scott-Gross Symposium, pp. 113-118 (cf. 166).

102. M. Hall, The theory of groups, Macmillan, New York, 1959.

103. M. Hall and D. Wales, The simple group of order 604, 800, J. Algebra 9 (1968), 417-450.

104. P. Hall, A note on soluble groups, J. London Math. Soc. 3 (1928), 98-105.

105. _ A characteristic property of soluble groups, J. London Math. Soc. 12 (1937), 190-200.

106. On the Sylow systems of a soluble group, Proc. London Math. Soc. 43 (1937), 316-323.

107. , On the system normalizers of a soluble group, Proc. London Math. Soc. 43 (1937), 507-528.

108. P. Hall and G. Higman, The p-length of a p-soluble group and reduction theorems for Burnside's problem, Proc. London Math. Soc. 7 (1956), 1-42.

109. K. Harada, On finite groups having self-centralizing 2-subgroups of small order, J. Algebra 33 (1975), 144-160.

110. __ On the simple group F of order $2^{14} \cdot 3^{6} \cdot 5^{6} \cdot 7 \cdot 11 \cdot 9$, Scott-Gross, symposium, pp. 119-195.

111. M. Harris, Finite groups with Sylow 2-subgroups of type PSp $(6, q)$, $q$ odd, Comm. Algebra 2 (1974), 181-232.

112. D. Held, The simple groups related to $M_{24}$, J. Algebra 13 (1969), 253-296.

113. C. Hering, W. Kantor and G. Seitz, Finite groups having a split $(B, N)$-pair of rank 1, J, Algebra 20 (1972), 435-475.

114. D. Higman, Finite permutation groups of rank 3, Math. Z. 86 (1964), 145-156.

115. D. Higman and C, Sims, A simple group of order 44, 352, 000, Math. Z. 105 (1968), 110-113.

116. G. Higman, Suzuki 2-groups, Illinois J. Math. 7 (1963), 79-96.

117. G. Higman and J. McKay, On Janko's simple group of order 50, 232, 960, Bull. London Math. Soc. 1 (1969), 89-94.

118. G. Higman and M. Powell, Instructional conference on finite simple groups, Oxford, 1969, Academic Press, London, 1971.

119. C. Ho, On the quadratic pair whose root group has order 3, Bull. Inst. Math. Acad. Sinica 1 (1973), 155-180.

120. Quadratic pairs for 3 whose root group has order greater than 3, I. Comm. Algebra 3 (1975), 961-1029.

121. - Chevalley groups of odd characteristic as quadratic pairs, J. Algebra 41 (1976), 202-211.

122. __ On the quadratic pairs, J. Algebra 43 (1976), 338-358.

123. —_, Quadratic pairs for odd primes, Bull. Amer. Math. Soc. 82 (1976), 941-943. 
124. D. Holt, Doubly transitive groups in which the stabilizer of two points is dihedral, Quart. J. Math. 27 (1976), 267-295.

125. , Transitive permutation groups in which a 2-central involution fixes a unique point (to appear).

126. B. Huppert, Endliche Gruppen. I, Springer-Verlag, Berlin and New York, 1967.

127. N. Ito, On a class of doubly transitive permutation groups, Illinois J. Math. 6 (1962), 341-352.

128. N. Iwahori, Centralizers of involutions in finite Chevalley groups, Seminar on algebraic groups, pp. 267-295 (cf. 50).

129. __ Finite groups, Symposium, Japan Soc. for Promotion of Science, Tokyo, 1976.

130. Z. Janko, $A$ new finite simple group with abelian 2-Sylow subgroups and its characterization, J. Algebra 3 (1966), 147-186.

131. $\_$, Some new simple groups of finite order. I, Ist. Naz. Alta Math. Symposia Math. 1 (1968), 25-64.

132. , The nonexistence of a certain type of simple group, J. Algebra 18 (1971), 245-253.

133. , A characterization of the Mathieu simple groups. I, II, J. Algebra 9 (1968), 1-19, $20-41$.

134. __ A new finite simple group of order $86,775,571,046,077,562,880$ which possesses $M_{24}$ and the full cover of $M_{22}$ as subgroups, J. Algebra 42 (1976), 564-596.

135. Z. Janko and J. Thompson, On a class of finite simple groups of Ree, J. Algebra 4 (1966), 274-292.

136. W. Kantor and G. Seitz, Some results on doubly transitive groups, Invent. Math. 13 (1971), 125-142.

137. L. Kovacs and B. Neumann, Proceedings of First International Conference on the Theory of Groups, (Canberra, 1965), Gordon and Breach, New York, 1967.

138. J. Leech, Some sphere packings in higher space, Canad. J. Math. 16 (1964), 657-682.

139. __ Notes on sphere packings, Canad. J. Math. 19 (1967), 251-267.

140. G. Leon and C. Sims, The existence and uniqueness of a simple group generated by $\{3,4\}$-transpositions, Bull. Amer. Math. Soc. 83 (1977), 1039-1040.

141. R. Lyons, Evidence for a new finite simple group, J. Algebra 20 (1972), 540-569.

142. A. MacWilliams, On 2-groups with no normal abelian subgroups of rank 3 and their occurrence as Sylow 2-subgroups of finite simple groups, Trans. Amer. Math. Soc. 150 (1970), 345-408.

143. D. Mason, Finite groups with Sylow 2-subgroup, the direct product of a dihedral and a wreathed group and related problems, Proc. London Math. Soc. 33 (1976), 401-442.

144. P. McBride, Nonsolvable signalizer functors on finite groups (to appear).

145. J. McKay, Computing with finite simple groups, 2nd International conference on the theory of groups, pp. 448-451 (cf. 51).

146. J. McKay and D. Wales, The multipliers of the simple groups of order 604, 800 and 50, 232, 960, J. Algebra 17 (1971), 262-272.

147. J. McLaughlin, Some subgroups of $S L_{n}\left(F_{2}\right)$, Illinois J. Math. 13 (1969), 108-115.

148. __ A simple group of order 898, 128, 000, Brauer-Sah, Symposium, pp. 109-111.

149. R. Niles, Pushing up $L_{2}\left(2^{n}\right)$, Ph.D. thesis, University of Chicago, 1976.

150. S. Norton, Some properties of the Fischer monster (unpublished).

151. M. O'Nan, Automorphisms of unitary block designs, J. Algebra 20 (1972), 495-511.

152. _ A characterization of $U_{3}(q)$, J. Algebra 22 (1972), 254-296.

153. _ A characterization of $L_{k}(q)$ as a permutation group, Math. Z. 127 (1972), 301-314.

154. _ـ Normal structure of the one-point stabilizer of a doubly transitive permutation group, Trans. Amer. Math. Soc. 214 (1975), 1-42, 43-74.

155. _ Some evidence for the existence of a new simple group, Proc. London Math. Soc. 32 (1976), 421-479.

156. __ Local properties of sporadic groups (unpublished).

157. D. Parrott, A characterization of the Tits simple group, Canad. J. Math. 24 (1972), 672-685.

158. N. Patterson, On Conway's group .0 and some subgroups (to appear).

159. K. Phan, On groups generated by special unitary groups. I, II, J. Austral. Math. Soc. 23 (1977), 67-77, 129-146.

160. R. Ree, $A$ family of simple groups associated with the simple Lie algebra $F_{4}$, Amer. J. Math. 83 (1961), 401-420. 
161. , A family of simple groups associated with the simple Lie algebra $G_{2}$, Amer. J. Math. 83 (1961), 432-462.

162. Classification of involutions and centralizers of involutions, Kovacs, Neumann, pp. 281-301 (cf. 137).

163. A. Rudvalis, Evidence for the existence of a simple group of order 145, 926, 144, 000 (to appear).

164. I. Schur, Untersuchen über die Darstellung der endlichen Gruppen durch gebrochnen linearen Substitutionen, Crelle J. 132 (1907), 85-137.

165. __ Über die Darstellungen der symmetrischen und alternierenden Gruppen durch gebrochene lineare Substitutionen, Crelle J. 139 (1911), 155-250.

166. W. Scott and F. Gross, Proc. of conference on finite groups, Academic Press, New York, 1976.

167. G. Seitz, Generation of finite groups of Lie type (to appear).

168. E. Shult, On a class of doubly transitive groups, Illinois J. Math. 16 (1972), 434-455.

169. __ On the fusion of an involution in its centralizer (to appear).

170. E. Shult, T. Gagen and M. Hale, Gainesville conference on finite groups, North-Holland, Amsterdam; American Elsevier, New York, 1973.

171. C. Sims, Graphs and finite permutation groups. I, II, Math. Z. 95 (1967), 76-86; 103 (1968), 276-281.

172. , The existence and uniqueness of Lyons' group, Gainesville conference, 138-141 (cf. 170).

173. , The existence and uniqueness of $O$ 'Nan's group (unpublished).

174. F. Smith, Finite groups whose Sylow 2-subgroups are the direct product of a dihedral and semidihedral group, Illinois J. Math. 17 (1973), 352-386.

175. __ Finite groups whose Sylow 2-subgroups are the direct product of two semidihedral groups, Illinois J. Math. 17 (1973), 387-396.

176. __ A characterization of the Conway simple group .2, J. Algebra 31 (1974), 91-116.

177. S. Smith, Finite group with a maximal 2-local subgroup in which $\mathrm{O}_{2}$ is extra-special. I, II, III (to appear).

178. R. Solomon, Finite groups with intrinsic 2-components of type $\hat{A}_{n}$, J. Algebra 33 (1975), 498-522.

179. __ Maximal 2-components in finite groups, Comm. Algebra 4 (1976), 561-594.

180. _ 2-signalizers in groups of alternating type, Comm. Algebra 6 (1978), 529-549.

181. R. Stanton, The Mathieu groups, Canad. J. Math. 3 (1951), 164-174.

182. B. Stark, Quadratic pairs revisited (to appear).

183. R. Steinberg, Variations on a theme of Chevalley, Pacific J. Math. 9 (1959), 875-891.

184. , Automorphisms of finite linear groups, Canad. J. Math. 12 (1960), 606-615.

185. __ Générateurs, relations, et revêtements de groupes algébriques, Colloque sur la théorie des groupes algébriques, C.B.R.M., Brussels, 1962, 113-127.

186. __ Lectures on Chevalley groups, Lecture notes, Yale University, 1967-1968.

187. M. Suzuki, The nonexistence of a certain type of simple group of odd order, Proc. Amer. Math. Soc. 8 (1957), 686-695.

188. __ Finite groups with nilpotent centralizers, Trans. Amer. Math. Soc. 99 (1961), 425-470. , On a class of doubly transitive groups. I, II, Ann. of Math. 75 (1962), 105-145; 79 (1964), 514-589.

190. __ On characterizations of linear groups. III, Nagoya J. Math. 21 (1962), 159-183.

191. __ A characterization of the 3-dimensional projective unitary group over a finite field of odd characteristic, J. Algebra 2 (1965), 1-14.

192. __ A simple group of order 448, 345, 497, 600, Brauer-Sah, Symposium, 113-119.

193. __ Characterizations of linear groups, Bull. Amer. Math. Soc. 75 (1969), 1043-1091.

194. _ Characterizations of some finite simple groups, Internat. Congr. Math. Nice 1 (1971), 371-373.

195. _ A characterization of the orthogonal groups over finite fields of characteristic two, Iwahori, Symposium, pp. 105-112.

196. J. Tate, Quotient groups of finite groups, Topology 3, (1964), supp. 1, pp. 109-111.

197. J. Thompson, Normal p-complements for finite groups, Math. Z. 72 (1960), 332-354.

198. , 2-signalizers of finite groups, Pacific J. Math. 14 (1964), 363-364. 
199.

Nonsolvable finite groups all of whose local subgroups are solvable. I-VI, Bull. Amer. Math. Soc. 74 (1968), 383-437; Pacific J. Math. 33 (1970), 451-536; 39 (1971), 483-534; 48 (1973), 511-592; 50 (1974), 215-297; 51 (1974), 573-630.

200. _ Toward a characterization of $E_{2}^{*}(q)$. I, II, III, J. Algebra 7 (1967), 406-414; 20

(1972), 610-621; 49 (1977), 162-166.

201. __ Notes on the B-conjecture (unpublished).

202. __ Fixed points of p-groups acting on p-groups, Math. Z. 86 (1964), 12-13.

203. __ Quadratic pairs (unpublished).

204. __ Simple 3'-groups, Symposia Math. XIII, Academic Press, London, 1974, pp. 517-530; balance (unpublished).

205. __ A simple subgroup of $E_{8}$ (3), Iwahori, Symposium, pp. 113-116 (cf. 129).

206. $\longrightarrow$ A conjugacy theorem for $E_{8}$, J. Algebra 38 (1976), 525-530.

207. F. Timmesfeld, Eine Keunzeichnung der linearen Gruppen über GF(2), Math. Ann. 189 (1970), 134-160.

208. __ Groups generated by root involutions. I, J. Algebra 33 (1975), 75-135.

209. __ Groups with weakly closed T. I. subgroups, Math. Z. 143 (1975), 243-278.

210. J. Tits, Théorème de Bruhat et sous-groupes paraboliques, C. R. Acad. Sci. Paris 254 (1962), 2910-2912.

211. G_ Groupes simples et géométries associées, Proc. Internat. Congr. Math. Stockholm (1962), pp. 197-221.

212. _ Algebraic and abstract simple groups, Ann. of Math. 80 (1964), 313-329.

213. _ Buildings of spherical type and finite $(B, N)$-pairs, Springer-Verlag, Berlin and New York, 1974.

214. J. Walter, The characterization of finite groups with abelian Sylow 2-subgroups, Ann. of Math. 89 (1969), 405-514.

215. H. Ward, On Ree's series of simple groups, Trans. Amer. Math. Soc. 121 (1966), 62-89.

216. , On the triviality of primary parts of the Schur multiplier, J. Algebra 10 (1968), $377-382$.

217. A. Weir, Sylow p-subgroups of the classical groups over finite fields with characteristic prime to $p$, Proc. Amer. Math. Soc. 6 (1955), 529-533.

218. H. Wielandt, Primitive Permutationsgruppen vom Grad 2p, Math. Z. 63 (1956), 478-485.

219. __ Finite permutation groups, Academic Press, New York, 1964.

220. W. Wong, Determination of a class of primitive permutation groups, Math. Z. 99 (1967), 235-246.

221. , On finite groups whose 2-Sylow subgroups have cyclic subgroups of index 2, J. Austral. Math. Soc. 4 (1964), 90-112.

222. H. Zassenhaus, Kennzeichnung endlicher linearer Gruppen als Permutationsgruppen, Abh.

Math. Sem. Hamburg Univ. 11 (1936), 17-40.

223. C. Curtis, Irreducible representations of finite groups of Lie type, Crelle J. 219 (1965), 180-199.

224. W. Feit and J. Thompson, Finite groups which contain a self-centralizing subgroup of order 3, Nagoya Math. J. 21 (1962), 185-197.

225. G. Glauberman, Failure of factorization in p-solvable groups, Quart. J. Math. 24 (1973), 71-77.

226. , A pushing up theorem for $L_{2}\left(2^{n}\right)$ (to appear).

227. E. Mathieu, Mémoire sur le nombre de valeurs que peut acquérir une fonction quand on $y$ permute ses variables de toutes les manières possibles, Crelle J. 5 (1860), 9-42.

228. __ Mémoire sur l'étude des functions de plusieures quantités, sur la manière de les formes et sur les substitutions qui les laissent invariables, Crelle J. 6 (1861), 241-323.

229. ___ Sur la function cinq fois transitive des 24 quantités, Crelle J. 18 (1873), 25-46.

230. S. Norton, Construction of Harada's group, Ph.D. thesis, University of Cambridge, 1976.

231. F. Smith, Transitive permutation groups in which a 2-central involution fixes a unique point (unpublished).

232. W. Tutte, A family of cubical graphs, Proc. Cambridge Philos. Soc. 43 (1947), 459-474.

233. __ On the symmetry of cubic graphs, Canad. J. Math. 11 (1959), 621-624.

234. R. Griess, On a subgroup of order $2^{15}|G L(5,2)|$ in $E_{8}(C)$, J. Algebra 40 (1976), 271-279.

Department of Mathematics, Rutgers University, New Brunswick, New Jersey 08903 
